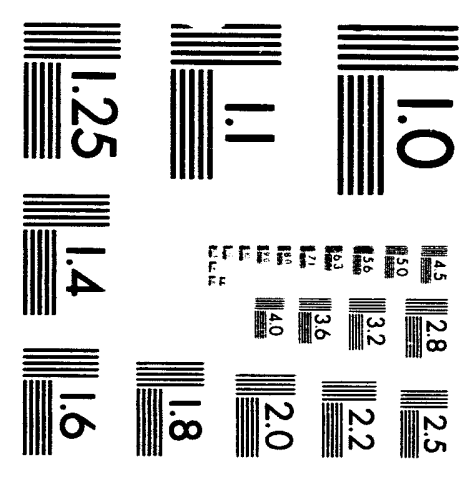



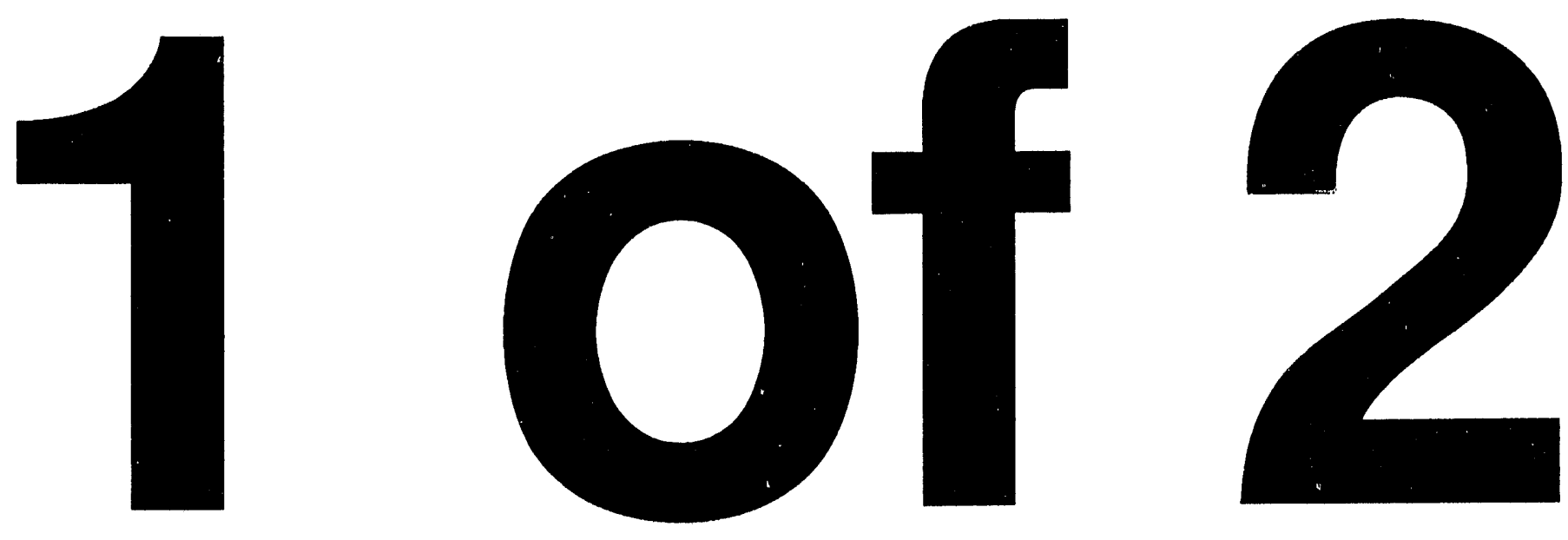


$$
\text { DOE/OR/00033-T564 }
$$

\section{RESONANT HOLOGRAPHIC MEASUREMENTS OF LASER ABLATION PLUME EXPANSION IN VACUUM AND ARGON GAS BACKGROUNDS}

by

Roger Alan Lindley

A dissertation submitted in partial fulfillment of the requirements for the degree of

Doctor of Philosophy

(Nuclear Engineering)

in The University of Michigan

1993

Doctoral Committee:

Professor Ronald Gilgenbach, Chair

Associate Professor Mary Brake

Professor Terry Kammash

Professor Yue Lau

Professor Duncan Steel 


\section{DISCLAIMER}

This report was prepared as an account of work sponsored by an agency of the United States Government. Neither the United States Government nor any agency thureof, nor any of their employees, makes any warranty, express or implied, or assumes any legal liability or responsibility for the accuracy, completeness, or usefulness of any information, apparatus, product, or process disclosed, or represents that its use would not infringe privately owned rights. Reference herein to any specific commercial product, process, or service by trade name, trademark, manufacturer, or otherwise does not necessarily constitute or imply its endorsement, recommendation, or favoring by the United States Government or any agency thereof. The views and opinions of authors expressed herein do not necessarily state or reflect those of the United States Government or any agency thereof. 


\section{C.Roger Alan Lindley 1993 \\ All Rights Reserved}

The Government reserves for itself and others acting on its behalf a royalty free, nonexclusive, irrevocable, world-wide license for governmental purposes to publish, distribute, translate, duplicate, exhibit, and perform any such data copyrighted by the contractor. 
This dissertation is dedicated to my Master, Jesus Christ, of whom I am greatly indebted. It has been by His leading that I have studied at the University of Michigan and performed this work. 


\section{ACKNOWLEDGEMENTS}

First and foremost, I wish to acknowledge the steady guidance of my advisor, Professor Ronald Gilgenbach. I greatly appreciate his commitment to the development of professional, scientific skills and the graduation of his students.

I would like to acknowledge Professor Duncan Steel giving generously of his time to help me through multiple drafts of the theory

section. Without his help, I would still be lost in the Forest of Statistical Optics. Also, I would like to acknowledge Professor Lau's contribution of proposing to use the saddle point theorem to solve the otherwise Unsolvable Integral (located near the exit of the aforementioned forest). Without this one step, the theory of resonant hologram interpretation would not have been extended in this work.

A special thanks to Professor James Holloway for being on the evaluating committee at the defense, filling in for Professor Terry Kammash and Professor Mary Brake who were both unable to be at the defense. Also, I would like to thank Professor Holloway for explaining to me the finer points of solving the roots of equations using Mathematica.

I would like to thank all of the professors on my committee for reviewing my dissertation on short notice, and for having a good balance of light-hearted laughter and intense questioning at my defense. 
I would like to acknowledge Peter Ventzek, who taught me the ropes of laser ablation, as well as Hong (More Data!) Ching and Joel (On Patrol) Lash, who are also a part of the Laser Ablation Lab Group. I would like to thank the others of Plasma Bay for their friendship and assistance: Professor Mary Brake, Professor Ward Getty, Bob Bosch, Cindy Brooks, Melisa Buie, Jin Choi, Joe Geddes, Kevin Junck, Peter Menge, Will McColl, Mike Passow, Kelly Pearce, Jerry Pender, Ron Spears, Tom Spencer, Ron Tempske, and Mark Walter.

I would also like to thank the undergraduate students who have been a part of the Laser Ablation Lab Group during my stay: Aaron Allen, Antwan Edson, Derik Love, Ross Schmitz, and Orlando Valree. Not only do I appreciate their help with the experiments, but also each has helped to make Plasma Bay a safer and more organized place to work.

Thank you to Jerry Pender and Arjun Selvakumar for taking the four point resistive probe measurements in the clean room of the Solid State Electronics Laboratory of the Electrical Engineering and Computer Science Department, and thank you to Professor Jessica Schwartz of the Physiology Department for the use of her microbalance.

I would like to thank my better half, Rachel, for all of her love, patience and hard work during our stay at the University of Michigan. I really appreciate all the behind the scenes effort that she has put in to make this dissertation possible.

This work has been supported by National Science Foundation Grant CTS-9108971, and I have been supported by the Department of Energy's Magnetic Fusion Energy Technology Fellowship, which is administered through Oak Ridge Associated Universities. 


\section{TABLE OF CONTENTS}

DEDICATION

ACKNOWLEDGEMENTS....................................... iii

LIST OF FIGURES

LIST OF TABLES ......................................... xii

LIST OF APPENDICES .................................... xiii CHAPTER

1. INTRODUCTION ..................................... 1

Introduction To Laser Ablation ........................................... 1

Applications Of Laser Ablation ........................................... 4

The Study Of Plume Expansion ............................................ 9

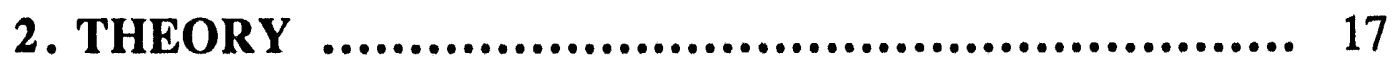

Holographic Interferometry ............................................ 17

Resonant Holographic Interferometry .............................. 21

Accounting For Finite Laser Bandwidth ............................ 25

The Solution for Doppler Broadening

And Finite Bandwidth .............................................. 28

3. EXPERIMENTAL CONFIGURATION................... 42

The Main Optical Table ....................................................... 42

The Lumonics Laser Spot Shape ........................................ 47

Developing And Reconstructing The Holograms................... 50

Plume Expansion In RF/Plasma Environments .................... 51 
4. EXPERIMENTAL RESULTS AND DISCUSSION .... 54

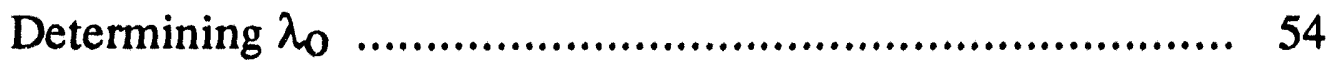

Resonant Refraction Effects ........................................ 56

Fringe Shift Interpretation ...................................... 58

Shot-To-Shot Consistency ....................................... 61

Laser Ablation In Vacuum

And Low Pressure, Inert, Background Gas .................... 65

Theoretically Modeling Plume Expansion In Vacuum

And Low Pressure, Inert, Background Gas .................... 95

Laser Ablation In Higher Pressure, Inert, Background Gas

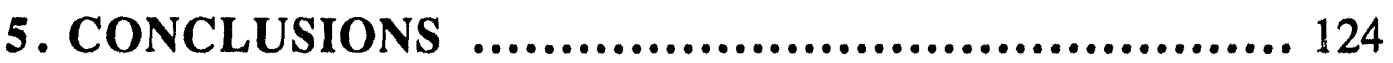

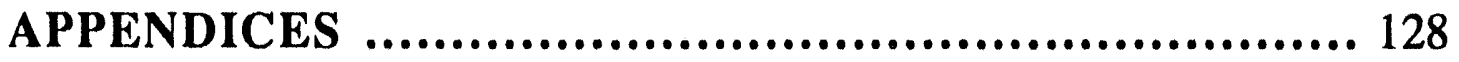

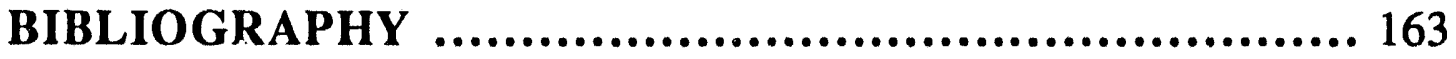




\section{LIST OF FIGURES}

\section{Eigure}

1.1. Schematic of laser ablation and laser ablation deposition

2.1. Schematic for recording the hologram ................... 18

2.2. Schematic for reconstructing the hologram ............... 18

2.3. The basic response curve for $W(\mathrm{z})$...................... 24

2.4. Fringe shift per line-density interpretation curve for $0.1 \mathrm{eV}, 0.3 \mathrm{eV}$ and $1.0 \mathrm{eV}$..................... 36

2.5. Comparison of the dye laser bandwidth and the resonant hologram interpretation curves .........

2.6. Fringe shift interpretation at $0.003 \mathrm{~nm}$ for the $\mathrm{Al}(\mathrm{I}), 394.401 \mathrm{~nm}$ line for $0.3 \mathrm{eV} \ldots \ldots \ldots \ldots \ldots \ldots . .38$

2.7(a). Fringe shift interpretation at $0.003 \mathrm{~nm}$ for the $\mathrm{Al}(\mathrm{I}), 394.401 \mathrm{~nm}$ line for $0.1 \mathrm{eV}$, $0.3 \mathrm{eV}, 1.0 \mathrm{eV}$, and $3.0 \mathrm{eV}$; Measures'

2.7(b). Fringe shift interpretation at $0.003 \mathrm{~nm}$ for the $\mathrm{Al}(\mathrm{I}), 394.401 \mathrm{~nm}$ line for $0.1 \mathrm{eV}$, $0.3 \mathrm{eV}, 1.0 \mathrm{eV}$, and $3.0 \mathrm{eV}$, This Work

3.1(a). Experimental configuration of the main optics table.....

3.1(b). Experimental configuration of the main optics table, continued

3.2. Example laser ablation spots............................... 48

3.3. Schematic for reconstructing the hologram ................ 51 
3.4. Schematic of the RF-argon-piasma setup................... 53

4.1. Calibrating the dye laser to the aluminum line at $394.401 \mathrm{~nm}$

4.2. Refraction effects of the dye laser beam encruntering an ablation plume with $(n>1)$ and $(n<1)$

4.3. The method of fringe shift interpretation 59

4.4. Ten holograms taken at $\Delta \lambda=-0.013 \mathrm{~nm}$ in vacuum to demonstrate shot-to-shot consistency

4.5. Line-density versus wavelength at $0.5 \mu$ s for two fluences to show consistency of the line-density measurement as a function of wavelength

4.6(a-d). Time scan in vacuum 66

4.6(e-h). Time scan in vacuum, continued 67

4.6(i-j). Time scan in vacuum, continued 68

4.7(a). Total number of aluminum neutral atoms versus time in vacuum

4.7(b). Expansion of the $1 \times 10^{14} \mathrm{~cm}^{-2}$ equicontour line in vacuum

4.8(a-d). Time scan in vacuum, deposition case 71

4.8(e-h). Time scan in vacuum, deposition case, continued 72

4.8(i-1). Time scan in vacuum, deposition case, continued 73

4.8(m-t). Time scan in vacuum, deposition case, continued, dye-laser-resonance-absorption-photographs

4.9. Expansion of the $1 \times 10^{14} \mathrm{~cm}^{-2}$ equicontour line in vacuum, deposition case 
4.10. A photograph of the laser ablation aluminum thin film on the silicon substrate

4.11. A drawing of the deposited thin film overlayed by the ablating laser spot

4.12(a-d). Time scan in 14 mTorr argon gas ....................... 83

4.12(e-f). Time scan in 14 mTorr argon gas, continued............ 84

4.13(a). Total number of aluminum neutral atoms versus time in 14 mTorr argon gas ............................... 85

4.13(b). Expansion of the $1 \times 10^{14} \mathrm{~cm}^{-2}$ equicontour line in 14 mTorr argon gas

85

4.14(a-d). Time scan in $52 \mathrm{mT}$ Torr argon gas ........................ 86

4.14(e-h). Time scan in 52 mTorr argon gas, continued .......... 87

4.15(a). Total number of aluminum neutral atoms versus time in 52 mTorr argon gas ................................ 88

4.15(b). Expansion of the $1 \times 10^{14} \mathrm{~cm}^{-2}$ equicontour line in $52 \mathrm{mTorr}$ argon gas 88

4.16(a-d). Time scan in 210 mTorr argon gas 89

4.16(e-h). Time scan in 210 mTorr argon gas, continued.......... 90

4.16(i-j). Time scan in 210 mTorr argon gas, continued ........... 91

4.17(a). Total number of aluminum neutral atoms versus time in 210 mTorr argon gas

4.17(b). Expansion of the $1 \times 10^{14} \mathrm{~cm}^{-2}$ equicontour line in 210 mTorr argon gas

4.18(a). Total number of aluminum neutral atoms versus time in vacuum and lower pressure argon gas backgrounds 
4.18(b). Expansion of the $1 \times 10^{14} \mathrm{~cm}^{-2}$ equicontour line in vacuum and lower pressure argon gas backgrounds.. 93

4.19. Expansion of the ablation plume center in vacuum and lower pressure argon gas backgrounds

4.20. Inferred center of mass velocity versus time for vacuum and lower pressure argon gas backgrounds ................. 98

4.21. Inferred center of mass velocity versus time for vacuum and lower pressure argon gas backgrounds ................ 98

4.22. Inferred parameter, $N_{o} T_{y}{ }^{-1 / 2}$ versus time for vacuum and lower pressure argon gas backgrounds

4.23. Inferred center of mass velocity versus time for vacuum and lower pressure argon gas backgrounds

4.24(a-d). Time Scan in 1 Torr Argon Gas 102

4.24(e-h). Time Scan in 1 Torr Argon Gas, Continued 103

4.24(i-1). Time Scan in 1 Torr Argon Gas, Continued 104

4.24(m-n). Time Scan in 1 Torr Argon Gas, Continued 105

4.25(a). Total number of aluminum neutral atoms versus time in 1 Torr argon gas ...

4.25(b). Expansion of the $1 \times 10^{14} \mathrm{~cm}^{-2}$ equicontour line in 1 Torr argon gas

4.26(a-d). Time scan in 1 Torr argon RF/plasma 109

4.26(e-h). Time scan in 1 Torr argon RF/plasma, continued....... 110

4.26(i-1). Time scan in 1 Torr argon RF/plasma, continued.......... 111

4.26(m-n). Time scan in 1 Torr argon RF/plasma, continued...... 112

4.27(a). Total number of aluminum neutral atoms versus time in 1 Torr argon $\mathrm{RF} /$ plasma 
4.27(b). Expansion of the $1 \times 10^{14} \mathrm{~cm}^{-2}$ equicontour line in 1 Torr argon RF/plasma

4.28(a-d). Time scan in 35 Torr argon gas .......................... 115

4.28(e-h). Time scan in 35 Torr argon gas, continued ............. 116

4.28(i-j). Time scan in 35 Torr argon gas, continued .............. 117

4.28(k-n). Time scan in 35 Torr argon gas, continued................ 118

4.29. Expansion of the $1 \times 10^{14} \mathrm{~cm}^{-2}$ equicontour line in 35 Torr argon gas

4.30(a). Total number of aluminum neutral atoms versus time in vacuum and argon background gas; early times

4.30(b). Total number of aluminum neutral atoms versus time in vacuum and argon background gas; later times

4.31(a). Expansion of the $1 \times 10^{14} \mathrm{~cm}^{-2}$ equicontour line in 1 Torr and 35 Torr argon background gas; early times 122

4.31(b). Expansion of the $1 \times 10^{14} \mathrm{~cm}^{.2}$ equicontour line in 1 Torr and 35 Torr argon background gas; later times 122

4.32. Approximate expansion velocities in vacuum and in argon gas backgrounds 


\section{LIST OF TABLES}

\section{Table}

1.1(a). Diagnostics versus ablation target materials studied ..... 6

1.1(b). Diagnostics versus ablation target materials studied, continued

1.2. Target materials versus substrate materials ............... 8

1.3(a). Diagnostics versus parameters measured .................. 10

1.3(b). Diagnostics versus parameters measured, continued..... 11

2.1. Parameters For Resonant Hologram Interpretation ...... 38

3.1. Photographic developing process for the holographic plates ........................................... 50 


\section{LIST OF APPENDICES}

\section{Appendix}

1. Measures' theory .......................................................... 129

2. Calculating roots and fringe shifts ............................... 137

3. Fringe shift interpretation graphs ................................. 143

4. Time delay circuits .................................................. 151

5. Energy calibration …................................................... 155

6. Analyzing the laser ablation plume expansion ................. 159 


\section{CHAPTER 1 INTRODUCTION}

\section{Introduction to Laser Ablation}

Laser ablation is the removal of material from a solid surface by an energetic laser beam. Figure 1.1 shows a schematic of the basic process. An energetic laser beam is focused onto a solid surface where some of the energy is absorbed. Surface material is ejected away from the surface on the order of $10 \mathrm{~km} / \mathrm{s}$ due to mechanisms dependent upon the target

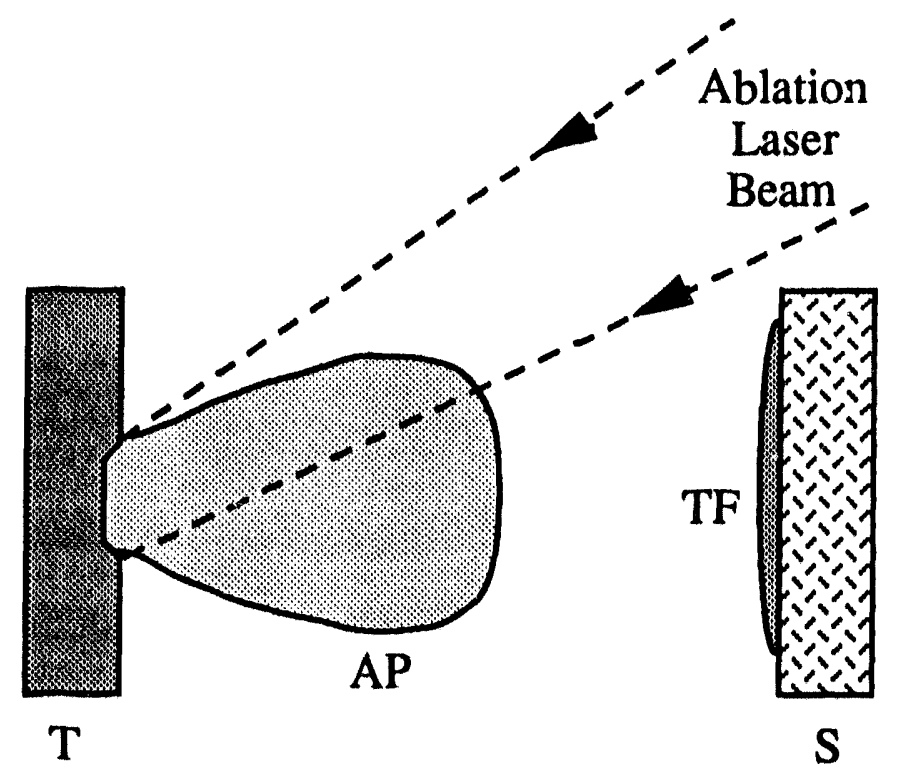

Fig. 1.1 Schematic of Laser Ablation and Laser Ablation Deposition. $\mathrm{T}=\mathrm{Target}, \mathrm{AP}=$ Ablation Plume, TF=Deposited Thin Film, and $S=$ Substrate. 
material. A shallow crater then remains on the target surface where the laser beam was incident. If there is a background gas of reasonable pressure ( $\geq 200 \mathrm{mT}$ Trr), the expanding vapor is inhibited by collisions with the background gas, causing condensation of the vaporized material as well as fluid dynamic vortex formations (e.g., a mushroom cloud shape) (Sap92). At lower pressures, the ablated material can then be collected on another surface by placing a substrate near the target. Generally, the substrate is heated to $>50^{\circ} \mathrm{C}$ to improve the material qualities of the deposited thin films.

The physical mechanisms removing material from the target surface vary with the type of target used. For the ablation of metals, the dominant mechanism is thermal heating, where the temperature of the target surface increases dramatically causing liquefication and vaporization (Dre91). A second ablation process for metals, observed under certain conditions, is called photochemical ablation (Dre91). In this process, the laser photons are absorbed by optically opaque electronic sites in the polycrystal structure of the metal. This energy then directly breaks the bonds of some of the atoms in the crystal, expelling them from the surface.

While the ablation of metals is dominated by the thermal ablation mechanism, both thermal ablation and photochemical ablation are significant mechanisms in the laser ablation of polymers (i.e., plastics). Certain sites on polymer chains are optically opaque and thus provide absorption sites in the lattice at which to break molecular bonds (Sri93, Dre86). For example, Srinivasan observed a considerable yield of MMA (methylmethacrylate) polymer chains in the ablation plume following the 
ablation of PMMA (polymethylmethacrylate) (Sri93). Both mechanisms were used to explain the observed plume composition.

For the ablation of ceramic materials, however, photochemical ablation has been shown to be the dominant mechanism (Dre86, Dic90). Defects in the inorganic lattice are optically opaque, providing locations for the laser energy to break lattice bonds, and it has been shown that an increase in the surface defect concentration increases the ablative material expulsion for the same laser ablation conditions (Dic90).

The physical mechanisms removing material from the target surface depend not only upon the target material but also on the laser fluence, the laser pulselength, and the laser wavelength. If the laser fluence is high enough and/or the laser pulselength is long enough, the ablation plume forms a hot, dense plasma, causing the laser energy to be absorbed by the plasma electrons via inverse bremsstrahlung. The absorbed laser energy is then transmitted to the target by heat conduction. This third ablation process is called plasma etching, although it is not truly separate from the thermal ablation process (Dre91).

Finally, the laser wavelength makes a difference in the ablation process. Ultraviolet (UV) lasers have photon energies (4.9 eV for 248 $\mathrm{nm}$ ) that are on the order of molecular bond energies (e.g., $\approx 1.9 \mathrm{eV}$ for $\mathrm{Al}-\mathrm{Al}$ and $\approx 3.6 \mathrm{eV}$ for $\mathrm{C}-\mathrm{C}$ ), making photochemical ablation processes more efficient. Visible and infrared (IR) lasers have lower energies per photon and thus require multiple-photon-absorption for the photochemical ablation mechanism. This in turn means that more of the laser energy is thermally absorbed by the target, that the effectiveness of photochemical ablation is reduced, and that the effectiveness of thermal ablation is enhanced (Bra91, Con89). However, there are two main 
drawbacks of using visible and especially IR lasers (Bra91). First of all, the rapidly, thermally heated region of the target is not confined to the laser spot. This causes unwanted surface damage (cratering) to the target when attempting to ablate desired patterns in the target surface (laser machining). Secondly, large debris is ejected from the surface instead of small fragments or single atoms. Not only is the large debris undesirable for making thin films on substrates, it also has a tendency to land on the target just beyond the laser spot. Again, this is undesirable for laser machining, especially for electronics. Therefore, UV lasers are preferred for both laser machining and laser ablation deposition.

\section{Applications of Laser Ablation}

As indicated above, the first application of laser ablation is the micromachining of metals, plastics, and ceramics, and the second application of laser ablation is the deposition of thin films onto substrates. Laser ablation is being intensely studied and used in the computer chip industry for drilling printed circuit boards (IBM), as a method of etching circuit patterns in photoresist (lithography) in preparation for plasma etching, etching circuit patterns in the semiconductor materials as a replacement for RF-plasma etching, and depositing semiconductor and superconductor materials onto silicon wafers. Micromachining is also useful in surgery, most notably for kidney stones (lithotripsy) and for eyes (corneal and keratorefractive surgery).

Laser ablation with very intense laser energies is being investigated as a possible $\mathrm{X}$-ray source for $\mathrm{X}$-ray lithography, as a possible commercial source of electricity by inducing fusion reactions (Inertial 
Confinement Fusion), and as a possible laser-gain medium for $X$-ray lasers. The production of $\mathrm{X}$-ray lasers is especially appealing to biologists since the electromagnetic absorption by proteins at a wavelength around $4 \mathrm{~nm}$ is much greater than for water (Elt90), thus allowing holographic observation of living cells and tissue. This would certainly revolutionize biological investigations that presently rely upon diagnostics requiring non-living samples. Laser ablation with very low laser intensities is already being utilized for nondestructive materials testing and the labeling/marking of plastic, metal, ceramic and glass surfaces via laser-induced surface effects.

Table 1.1(a) and (b) show a selection of materials versus diagnostics for which laser ablation plume expansion has been investigated and Table 1.2 shows a selection of targets and substrates that have been studied using laser ablation deposition. The diagnostics in Table 1.1 will be discussed in the next section.

The targets have been chosen either because they have a strong resonant transition at a wavelength accessible by the then-current-day dye laser technology or because of their present or possible future use in making electronic circuits. Barium $(\mathrm{Ba})$ and potassium $(\mathrm{K})$ are examples of the former. As examples of the latter, aluminum ( $\mathrm{Al})$, copper $(\mathrm{Cu})$, silicon ( $\mathrm{Si})$, silicon compounds, metallic oxides, metallic fluorides, and YBCO ( $\mathrm{YBa}_{2} \mathrm{Cu}_{3} \mathrm{O}_{7-x}$, a superconducting ceramic) are of interest in making thin films. In addition, the ablation of plastics (such as lucite, mylar, PET, PMMA, polyimide and polystyrene) is studied to develop better spatial control over the removal of printed circuit board insulating layers and photoresists in the process of making of computer chips. 
$a$

\begin{tabular}{|c|c|c|c|c|c|c|c|c|c|c|c|c|c|c|c|c|}
\hline & Al & $\mathrm{Ba}$ & $\mathrm{Cu}$ & $\mathbf{S i}$ & graphite & AlO & BN & YBCO & \begin{tabular}{c|}
$\mathrm{CaF}$ \\
$\mathrm{LiF}$ \\
$\mathrm{MoO}$
\end{tabular} & $\begin{array}{c}\mathrm{NaO} \\
\mathrm{SiO}\end{array}$ & GaAs & $\begin{array}{c}\text { Polyi- } \\
\text { mide }\end{array}$ & PET & PMMA & lucite & $\begin{array}{l}\text { poly- } \\
\text { sty- } \\
\text { rene }\end{array}$ \\
\hline $\begin{array}{l}\text { Ch.Part. } \\
\text { Collector }\end{array}$ & & & & & Mea88 & & & & & Dic90| & & & & & & \\
\hline $\begin{array}{c}\text { Langmuir } \\
\text { Probe }\end{array}$ & & & Gut89 & & & & & & & & & & & & & \\
\hline $\begin{array}{c}\text { TOF Mass } \\
\text { Spectro. }\end{array}$ & & & & & Cro91 & & & Geo89 & & & Wan92 & & & & & Fel88 \\
\hline $\begin{array}{l}\text { Laser } \\
\text { Defl. }\end{array}$ & & & & & \begin{tabular}{|c|} 
En187 \\
Mea88 \\
\end{tabular} & & & & Pet88 & & & Ven92b & $\begin{array}{l}\text { Sel91 } \\
\text { Ven90 } \\
\end{array}$ & Ven90| & Ven92b & \\
\hline $\begin{array}{l}\text { Abs. } \\
\text { Spectr. }\end{array}$ & & & Sap91 & & & & & $\begin{array}{l}\text { Che91 } \\
\text { Geo89 }\end{array}$ & & & & & & & & \\
\hline $\begin{array}{l}\text { Hook } \\
\text { Spectr. }\end{array}$ & & & Sap93 & & & & & & & & & & & & & \\
\hline $\begin{array}{l}\text { Em. } \\
\text { Spectr. }\end{array}$ & Rot84b & & & Rot84a & \begin{tabular}{|l|} 
Bra87 \\
Mea88
\end{tabular} & $\begin{array}{l}\text { Rot84a } \\
\text { Rot84b }\end{array}$ & Geog3 & $\begin{array}{l}\text { Geo93 } \\
\text { Zhe89 }\end{array}$ & & & & & & & & \\
\hline LIF & Wan91 & & Dre91 & & & Dre86 & & Oka92 & & & & & & & & \\
\hline PLIF & & $m^{\circ}$ & Sap92 & & & & & & & & & & & & & \\
\hline
\end{tabular}

Table 1.1(a) Diagnostics versus ablation target materials studied; a selection of articles investigating laser plume expansion. 


\begin{tabular}{|c|c|c|c|c|c|c|c|c|c|c|c|c|c|}
\hline & Al & $\mathrm{Ba}$ & $\mathrm{Cu}$ & $\mathrm{Na}$ & $\begin{array}{c}\mathrm{Ge} \\
\mathrm{MgO} \\
\mathrm{Si}\end{array}$ & BN & $\underset{\mathrm{Li} / \mathrm{Pb}}{\mathrm{K}}$ & YBCO & mylar & PET & PMMA & $\begin{array}{l}\text { polyi- } \\
\text { mide }\end{array}$ & AlO \\
\hline $\begin{array}{l}\text { R.\&T. } \\
\text { Scatt. }\end{array}$ & & Mos88 & Sap91 [ & & & & & & & & & & \\
\hline $\begin{array}{l}\text { Em. } \\
\text { Photo. }\end{array}$ & & & & & & $\begin{array}{l}\text { Geo } \\
93 \\
\end{array}$ & & $\begin{array}{l}\text { Geo92 } \\
\text { Geo93 }\end{array}$ & & & & & \\
\hline TOFPRL & & & & Bak87 & & & & & & & & & \\
\hline $\begin{array}{l}\text { Shadow } \\
\text {-graphy }\end{array}$ & & & & & & & & Gup91 & & & Sri93 & & \\
\hline $\begin{array}{l}\text { Absorption } \\
\text { Photography }\end{array}$ & \begin{tabular}{|c|} 
Gil91 \\
Ven \\
$92 \mathrm{a}$ \\
\end{tabular} & \begin{tabular}{|c|} 
Jel81 \\
Mos88 \\
Sie77 \\
\end{tabular} & & & & & & & Ven92a & & & Ven92a & \\
\hline Schlieren & Ven92a & & & & & & & & Ven92a & Ven90 & Ven90 & Ven92a & \\
\hline $\begin{array}{l}\text { Resonant } \\
\text { Schlieren }\end{array}$ & \begin{tabular}{|c} 
Jel81 \\
Koo79 \\
Mos88 \\
Sie77 \\
\end{tabular} & & & & & & & & & & & & \\
\hline $\begin{array}{c}\text { Micheison } \\
\text { Interfer- } \\
\text { ometry } \\
\end{array}$ & Wal86 & & Wal86 & & Wal86 & & & & & & Wal86 & Wal86 & Wal86 \\
\hline $\begin{array}{c}\text { Resonant } \\
\text { Holography }\end{array}$ & $\operatorname{Lin} 93$ & Koo79 & & & & & Dre75 & & & & & & \\
\hline
\end{tabular}

Table 1.1(b) Diagnostics versus ablation target materials studied, continued; a selection of articles investigating laser plume expansion. 


\begin{tabular}{|c|c|c|}
\hline Ref. & Target & Substrate \\
\hline Col 92 & Diamond & $\mathrm{Ti}$ \\
\hline Eri & $\begin{array}{l}\text { AlN,Co } \\
\text { Dy }\end{array}$ & $\mathbf{S i}$ \\
\hline Fog 90 & $\mathrm{SiO}$ & $\mathrm{Si}$ \\
\hline Kar 92 & YBCO & SrTiOO \\
\hline Koi 91 & $\begin{array}{l}\mathrm{CeO} \\
\mathrm{SrtiO}\end{array}$ & $\begin{array}{l}\text { Si and } \\
\text { SrTiO }\end{array}$ \\
\hline Kre 93 & $\begin{array}{l}\mathrm{Cu}, \mathrm{Fe} \\
\text { and } \mathrm{Co} \\
\text { alloys, } \\
\mathrm{PdSi} \\
\mathrm{CrTi} \\
\mathrm{Sn}\end{array}$ & $\begin{array}{l}\text { Si and } \\
\text { AlO }\end{array}$ \\
\hline Kwo 88 & YBCO & $\begin{array}{l}\text { SrTiO, Zr } \\
\text { MgO, } \\
\text { Sapphire }\end{array}$ \\
\hline Liu 92 & $\mathrm{BaCaCuO}$ & $\mathrm{MgO}$ \\
\hline
\end{tabular}

\begin{tabular}{|c|c|c|}
\hline Ref. & Target & Substrate \\
\hline Niu 92 & $\mathrm{SrCuO}$ & SrTiO \\
\hline Nor 91 & AlN & Sapphire \\
\hline Qia 93 & $(\mathrm{cBN})$ & Si \\
\hline Raj 93 & CoLZnTe & Ga-compounds \\
\hline Roy 92 & BaTiO & $\mathrm{Pt}$ \\
\hline Sat 88 & Graphite & Si, Quartz \\
\hline Sch 69 & $\begin{array}{l}\text { Cr, W, C } \\
\text { Ti, ZnS } \\
\text { SrTio } \\
\text { SbSZnS }\end{array}$ & Glass \\
\hline $\operatorname{Tar} 93$ & InO & $\begin{array}{l}\text { In-compounds } \\
\text { MgO, YSZ }\end{array}$ \\
\hline Ven 88 & YBCO & $\mathrm{Si}$ \\
\hline Zhu 93 & $\begin{array}{l}\mathrm{BiSrCa}- \\
\mathrm{CuO} \\
\mathrm{BiSrCuO}\end{array}$ & $\mathrm{MgO}$ \\
\hline
\end{tabular}

Table 1.2 Target materials versus substrate materials; a selection of articles investigating laser ablation deposition. 


\section{The Study of Plume Expansion}

This dissertation focuses on taking resonant holograms of expanding plumes from the laser ablation of solid aluminum targets and the subsequent calculation of line-integrated-densities of the neutral aluminum atoms in the ground state within the ablation plumes from those holograms. Since many different diagnostics have been used to study laser ablation plume expansion, a summary of the diagnostics utilized and the parameters that each measure are presented here.

Table 1.3(a) and (b) show various diagnostics versus the parameters they measure for a selection of articles investigating the expansion of laser ablation plumes. The simplest diagnostic is Charged Particle Collection, where the ablation particle current from a DCvoltage biased collection plate is measured as a function of time and bias voltage (Dic90, Mea88). This gives the total number of charged particles collected, here called the ion yield, as well as the time of flight (TOF) most-probable expansion velocity of the variously charged plume components, as selected by the bias voltage.

When the collection surface is instead the tip of a very small wire, charged particle collection is a Langmuir probe measurement (Gut89). Again, the collected current is measured as a function of DC-bias voltage and time, but here Langmuir probe theory can be used to calculate the particle density and the elcctron temperature of the ablation plume. Although the total ion yield is no longer measurable, the current as a function of time still gives charged particle TOF, most-probable expansion velocities. 


\begin{tabular}{|c|c|c|c|c|c|c|c|c|c|c|c|c|}
\hline & $\begin{array}{l}\text { species } \\
\text { recog. }\end{array}$ & $\begin{array}{l}\text { fluid } \\
\text { dyn. }\end{array}$ & $\begin{array}{l}\text { atomic } \\
\text { dens. }\end{array}$ & $\begin{array}{l}\text { dens. } \\
\text { grad. }\end{array}$ & $\begin{array}{c}\text { expan. } \\
\text { vel. }\end{array}$ & $\begin{array}{c}\text { kinetic } \\
\text { temp.-, } \\
\text { f(v) }\end{array}$ & $\begin{array}{l}\text { inter. } \\
\text { temp. }\end{array}$ & $\begin{array}{l}\text { elec. } \\
\text { temp. }\end{array}$ & $\begin{array}{l}\text { part. } \\
\text { yield }\end{array}$ & $\begin{array}{l}\text { thresh. } \\
\text { fluence }\end{array}$ & $\underset{\#}{\operatorname{mach}}$ & \begin{tabular}{|} 
thermal \\
diff.
\end{tabular} \\
\hline $\begin{array}{l}\text { Chg.Part. } \\
\text { Collector }\end{array}$ & Dic90 & & & & $\begin{array}{c}\text { Dic90 } \\
\text { Mea88 }\end{array}$ & & & & $\begin{array}{l}\text { Dic90 } \\
\text { Mea88 }\end{array}$ & & & \\
\hline Lang.probe & Gut89 & & Gut89 & & Gut89 & & & Gut89 & & & & \\
\hline $\begin{array}{c}\text { TOF Mass } \\
\text { Spectro. }\end{array}$ & $\begin{array}{l}\text { Cro91 } \\
\text { Fel87 }\end{array}$ & & & & $\begin{array}{l}\text { Cro91 } \\
\text { Fel87 }\end{array}$ & $\begin{array}{l}\text { Cro91 } \\
\text { Fel87 } \\
\end{array}$ & & & $\begin{array}{l}\text { Cro91 } \\
\text { Fel87 } \\
\text { Wan92 }\end{array}$ & & & \\
\hline $\begin{array}{l}\text { Laser } \\
\text { Defl. }\end{array}$ & $\begin{array}{l}\text { En187 } \\
\text { Mea88 }\end{array}$ & & $\begin{array}{l}\text { En187 } \\
\text { Ven90 }\end{array}$ & $\begin{array}{c}\text { En187 } \\
\text { Mea88 } \\
\text { Pet88 } \\
\text { Se191 } \\
\text { Ven90 } \\
\text { Ven92b }\end{array}$ & $\begin{array}{c}\text { Enl87 } \\
\text { Mea88 }\end{array}$ & & & & $\begin{array}{l}\text { Enl87 } \\
\text { Mea88 }\end{array}$ & $\begin{array}{c}\text { Mea88 } \\
\text { Pet88 } \\
\text { Sel91 }\end{array}$ & & Se191 \\
\hline $\begin{array}{c}\text { Abs. } \\
\text { Spectro. }\end{array}$ & $\begin{array}{l}\text { Che91 } \\
\text { Geo89 } \\
\text { Sap91 }\end{array}$ & & $\begin{array}{l}\text { Che91 } \\
\text { Sap91 }\end{array}$ & & $\begin{array}{l}\text { Che91 } \\
\text { Geo89 }\end{array}$ & Geo89 & & & & & & \\
\hline $\begin{array}{c}\text { Hook } \\
\text { Spectro. }\end{array}$ & Sap93 & & Sap93 & & & & & & & & & \\
\hline Em. Spectr. & $\begin{array}{l}\text { Bra87 } \\
\text { Geo93 } \\
\text { Mea88 } \\
\text { Rot84b } \\
\text { Zhe89 } \\
\end{array}$ & & & & $\begin{array}{l}\text { Bra87 } \\
\text { Geo89 } \\
\text { Mea88 } \\
\text { Zhe89 } \\
\end{array}$ & Zhe89 & Geo93 & $\begin{array}{l}\text { Bra87 } \\
\text { Mea88 }\end{array}$ & & Rot84b & Zhe89 & \\
\hline LIF & $\begin{array}{l}\text { Dre86 } \\
\text { Dre91 } \\
\text { Sap91 } \\
\text { Wan91 }\end{array}$ & & & & $\begin{array}{l}\text { Dre86 } \\
\text { Dre91 } \\
\text { Sap91 } \\
\text { Wan91 }\end{array}$ & $\begin{array}{l}\text { Dre86 } \\
\text { Dre91 } \\
\text { Wan91 }\end{array}$ & $\begin{array}{l}\text { Dre86 } \\
\text { Sap91 } \\
\text { Wan91 }\end{array}$ & & & & & \\
\hline PLIF & $\begin{array}{l}\text { Cap90 } \\
\text { Sap92 }\end{array}$ & $\begin{array}{l}\text { Cap90 } \\
\text { Sap92 }\end{array}$ & $\begin{array}{l}\text { Cap90 } \\
\text { Sap92 }\end{array}$ & Cap90 & $\begin{array}{l}\text { Cap90 } \\
\text { Sap92 }\end{array}$ & & & & & & & \\
\hline
\end{tabular}

Table 1.3(a) Diagnostics versus parameters measured. a selection of articles investigating laser ablation plume expansion. 


\begin{tabular}{|c|c|c|c|c|c|c|c|}
\hline & $\begin{array}{r}\text { species } \\
\text { recog. }\end{array}$ & $\begin{array}{l}\text { fluid } \\
\text { dyn. }\end{array}$ & $\begin{array}{l}\text { atomic } \\
\text { dens. }\end{array}$ & $\begin{array}{l}\text { elec. } \\
\text { dens. }\end{array}$ & $\begin{array}{l}\text { expan. } \\
\text { vel. }\end{array}$ & $\begin{array}{l}\text { kinetic } \\
\text { temp. } \\
\mathrm{f}(\mathrm{v})\end{array}$ & $\begin{array}{l}\text { init. } \\
\text { target } \\
\text { temp. }\end{array}$ \\
\hline $\begin{array}{c}\text { R. \& T. } \\
\text { Scatt. }\end{array}$ & $\begin{array}{l}\text { Mos88 } \\
\text { Sap91 }\end{array}$ & & Mos88 & & Sap91 & & \\
\hline Em. Photo. & $\begin{array}{l}\text { Geo92 } \\
\text { Ge093 }\end{array}$ & $\begin{array}{l}\text { Geo92 } \\
\text { Geo93 } \\
\end{array}$ & & & \begin{tabular}{|l|} 
Geo92 \\
Geo93 \\
\end{tabular} & & \\
\hline TOFPRL & & & & & Bak87 & Bak87 & \\
\hline $\begin{array}{l}\text { Shadow- } \\
\text { graphy }\end{array}$ & & $\begin{array}{l}\text { Gup91 } \\
\text { Sri93 }\end{array}$ & & & $\begin{array}{l}\text { Gup91 } \\
\text { Sri93 }\end{array}$ & & Gup91 \\
\hline $\begin{array}{l}\text { Abs. } \\
\text { Photo. }\end{array}$ & \begin{tabular}{|c|} 
Gil91 \\
Jel81 \\
Mos88 \\
Sie77 \\
Ven92a \\
\end{tabular} & \begin{tabular}{|c|} 
Gil91 \\
Jel81 \\
Mos88 \\
Sie77 \\
Ven92a \\
\end{tabular} & & & \begin{tabular}{|c|} 
Gil91 \\
Jel81 \\
Mos88 \\
Sie77 \\
Ven92a \\
\end{tabular} & & \\
\hline Schl. & & $\begin{array}{l}\text { Ven90 } \\
\text { Ven92a }\end{array}$ & & & $\begin{array}{l}\text { Ven90 } \\
\text { Ven92a }\end{array}$ & & \\
\hline Res. Schl. & \begin{tabular}{c|} 
Jel81 \\
Koo79 \\
Mos88 \\
Sie77 \\
\end{tabular} & $\begin{array}{c}\text { Jel81 } \\
\text { Koo79 } \\
\text { Mos88 } \\
\text { Sie77 } \\
\end{array}$ & & & \begin{tabular}{|c|} 
Jel81 \\
Koo79 \\
Mos88 \\
Sie77 \\
\end{tabular} & & \\
\hline Mich. Int. & & Wal86 & Wal86 & Wal86 & Wal86 & & \\
\hline Res. Holo. & $\begin{array}{l}\text { Dre75 } \\
\text { Koo79 } \\
\text { Lin 93 }\end{array}$ & $\begin{array}{l}\text { Dre75 } \\
\text { Koo79 } \\
\text { Lin93 }\end{array}$ & \begin{tabular}{l|} 
Dre75 \\
Koo79 \\
Lin93 \\
\end{tabular} & $\begin{array}{l}\text { Dre75 } \\
\text { Koo79 }\end{array}$ & $\begin{array}{l}\text { Dre75 } \\
\text { Koc79 } \\
\text { Lin93 }\end{array}$ & & \\
\hline
\end{tabular}

Table 1.3(b) Diagnostics versus parameters measured, continued; a selection of articles investigating laser ablation plume expansion. 
A TOF Mass Spectrometer placed in the path of the expanding ablation plume measures the TOF particle flux as a function of mass and time (Cro91, Fel87, Wan92). This allows the TOF most-probable expansion velocity, the velocity distribution, and thus the kinetic temperature to be determined as a function of mass.

If an unexpanded laser beam is passed through an ablation plume with strong density gradients, the laser beam will be deflected (Laser Deflection) (Enl87, Mea88, Pet88, Se191, Ven90, Ven92b). The amount of deflection can be used to measure the path-integrated density-gradient of the ablation plume. When cylindrical symmetry is assumed for the ablation plume, the particle density can also be calculated (En187).

When the wavelength of the unexpanded, laser beam is tuned near an atomic or molecular resonance and passed through an ablation plume with weak density gradients, the transmission as a function of wavelength can be measured (Absorption Spectroscopy) (Che91, Ge089, Sap91). The line-width and line-shape of the transmission signal can provide information concerning the line-broadening collisional mechanisms occurring in the ablation plume. This allows the atomic density to be calculated from the transmission signal at peak absorption. Density versus time then gives the TOF, most-probable expansion velocity, the velocity distribution, and thus the kinetic temperature of the plume.

If the unexpanded, dye laser beam passing through the ablation plume (with weak density gradients) has a wide bandwidth, the relative phase shift of the laser light as a function of wavelength can be measured (Hook Spectroscopy) (Sap93). A peak in the phase shift occurs on either side of a resonance line, so the resonant line-density along the beam path 
can be calculated from the wavelength separation of the two peak phase shifts.

During the ablation process, atoms and molecules are given kinetic energy while molecules are given rotational and vibrational energy (internal energy) as well. In addition, electronic states within the atoms are excited and some electrons are removed from their atoms. As the electrons and ions recombine and the excited electronic states relax, observable light is emitted. Emission Spectroscopy collects and measures the light emitted from a single point in space as a function of wavelength (Bra87, Ge093, Mea88, Rot84b, Zhe89). Light emitted at known atomic and molecular resonance wavelengths provides species-recognition and molecular internal-temperatures. The intensity of light at the peak of a resonance as a function of time provides the TOF, most-probable expansion velocity, the velocity distribution, the fraction of the ionic species and thus the kinetic temperature of the plume.

When a dye laser is focused onto the same point in space described above and the wavelength is tuned to the resonance of a plume constituent, an upward electronic transition can be induced in a large fraction of the ablated atoms and the intensity at a known relaxation wavelength can be observed versus time. This is the principle of Laser Induced Fluorescence (LIF), and it normally utilizes certain transitions in given atoms and ions where a strong relaxation signal can be observed at a wavelength other than the induced excitation wavelength (Dre86, Dre91, Sap91, Wan91). The measured emission signal versus time gives the TOF most probable velocity, the velocity distribution, and thus the kinetic temperature of the plume. In addition, emission spectroscopy can be done simultaneously at other wavelengths to obtain molecular-intemal- 
temperatures and the velocity measurements for other atomic constituents.

If a dye laser beam tuned to an LIF excitation wavelength is focused into a sheet of light (a plane) and passed through the plume, a photograph of the LIF-relaxation light emitted from this plume can be taken perpendicular to the plane of light (Planar Laser Induced Fluorescence, PLIF) (Cap90, Sap92). This provides expansion fluid dynamics and leading edge expansion velocities for the resonantly excited plume constituent.

When the dye laser light passes through the ablation plume, there is a small portion of photons that are radiatively scattered by the electrons (Thomson Scattering), elastically scattered by the atoms and molecules (Rayleigh Scattering) (Mos88), and inelastically scattered by the atoms and molecules (Raman Scattering). Sappey and Gamble (Sap91) have measured Thompson and Rayleigh scattering signals on top of their LIF signal and utilized them to determine the location and velocity of the electrons and molecules, respectfully, in their ablation plumes.

When a camera is situated to view the expansion of the ablation plume away from the target, the plume image can be captured on film or electronically stored using a CCD camera (Emission Photography) (Ge092, Ge093). This provides progressive photos of the fluid dynamics of the emitting species as well as a leading edge plume expansion velocity. It is especially useful for hot particulate matter from laser ablation.

Photo-Multiplier Tubes (PMT's) which measure the overall intensity of emitted light can be linearly arrayed with increasing distance from the target surface, while situated to view the ever-expanding plume in succession (TOF Plasma Recombination Light diagnostic) (Bak87). 
The total light signal from each PMT versus time can then be used to estimate the "center of mass velocity" away from the target and the kinetic temperature of the expanding plume in the center of mass reference frame.

The plume can also be photographed by using an expanded and collimated dye laser beam passing through the ablation plume and then imaged directly onto the filrn (Shadowgraphy) (Gup91, Sri93). Variations of dark and light on the film denote refraction by a shock wave, an ablation plume density gradient, or nonresonant absorption by dense plume particulate. When the dye laser is tuned to an atomic or molecular resonance, darkness on the film denotes the presence of resonant plume constituents as well. This has been called Absorption Photography (Mos88), Resonant Shadowgraphy (Sie77, Je181), or DyeLaser-Resonance-Absorption-Photography (DLRAP) (Gi191, Ven92).

The observation of the light refracted by the ablation plume can be enhanced by blocking the refracted light from reaching the film. For nonresonant laser wavelengths, this is called Schlieren Photography (Ven90, Ven92a), and for resonant laser wavelengths this is called Resonant Schlieren Photography (Je181, Koo'79, Mos88, Sie77).

Finally, interferometric methods may be utilized to observe ablation plumes. This entails splitting the laser beam into two components, expanding and collimating them both, passing one beam through the ablation plume (the object beam), and recombining the two beams to form an interference pattern. The total phase shift of the laser light passing through the ablation plume is measured as a function of location on the recorded interferogram. By taking three interferograms at three different wavelengths, one near a resonant transition and two 
away from the resonance, the source of the phase shift can be determined and thus give: 1) the electron line-density, 2) the nonresonant atomic or molecular line-density, and 3) the resonant constituent atomic (or ionic) line-density. Of course, the photographic images of the plume expansion provide information concerning the fluid dynamics and the expansion velocities of the three, measurable components.

There are three types of interferometry that can be used to study plume expansion. If the object beam passes through the ablation plume once and the two beams are recombined to be collinear, it is called MachZehnder Interferometry. If the object beam passes through the ablation plume twice by reflection off a mirror and the two beams are recombined to be collinear, it is called Michelson Interferometry. If, 1) the object beam passes through the plume once, 2 ) the two beams are recombined on a photographic emulsion, and 3) the laser is pulsed once with the ablation plume present, and once without the plume, it is called Holographic Interferometry. As of this writing, one laboratory is known to have utilized nonresonant Michelson interferometry (Wal86) to study laser ablation plume expansion, and two other laboratories are known to have utilized resonant holographic interferometry (Dre75, Koo79).

A derivation and calculation of an extension to the theory of resonant holographic interferometry, for use in the interpretation of resonant holograms at wavelengths very near an atomic resonance, will be presented in Chapter 2. The experimental configuration will be described in Chapter 3. The resonant holograms of the expanding ablation plumes and their interpretation will be presented in Chapter 4, and the overall conclusions of this work will be presented in Chapter 5. 


\section{CHAPTER 2}

\section{THEORY}

\section{Holographic Interferometry}

When light is incident upon a neutral gas, it will interact with the gas with some combination of absorption, reflection, refraction, and phase shift. If the absorption is due to a resonant interaction with a quantum mechanical transition in an atomic or molecular component of the gas, then the light will be re-emitted in a random direction with respect to that gas particle. If the gas is relatively transparent, the lineintegrated-density of the gas can be determined by measuring the phase shift of a laser beam passing through the gas. With a tunable dye laser, the laser wavelength can be tuned very near a resonant transition of one species of the gas and, if resonant absorption is not excessive, the lineintegrated-density of the aforementioned gas species can be measured with several orders of magnitude more sensitivity than with a nonresonant wavelength.

Holographic interferometry provides an accurate, photographic method to measure the incurred phase shift of the laser beam after it has passed through a transparent medium. There are a number of good textbooks that present detailed discussions of holographic interferometry, including Vest (Ves85) and Hecht (Hec79). Figure 2.1 shows a schematic of a holographic interferometry setup. The laser beam is split into two 


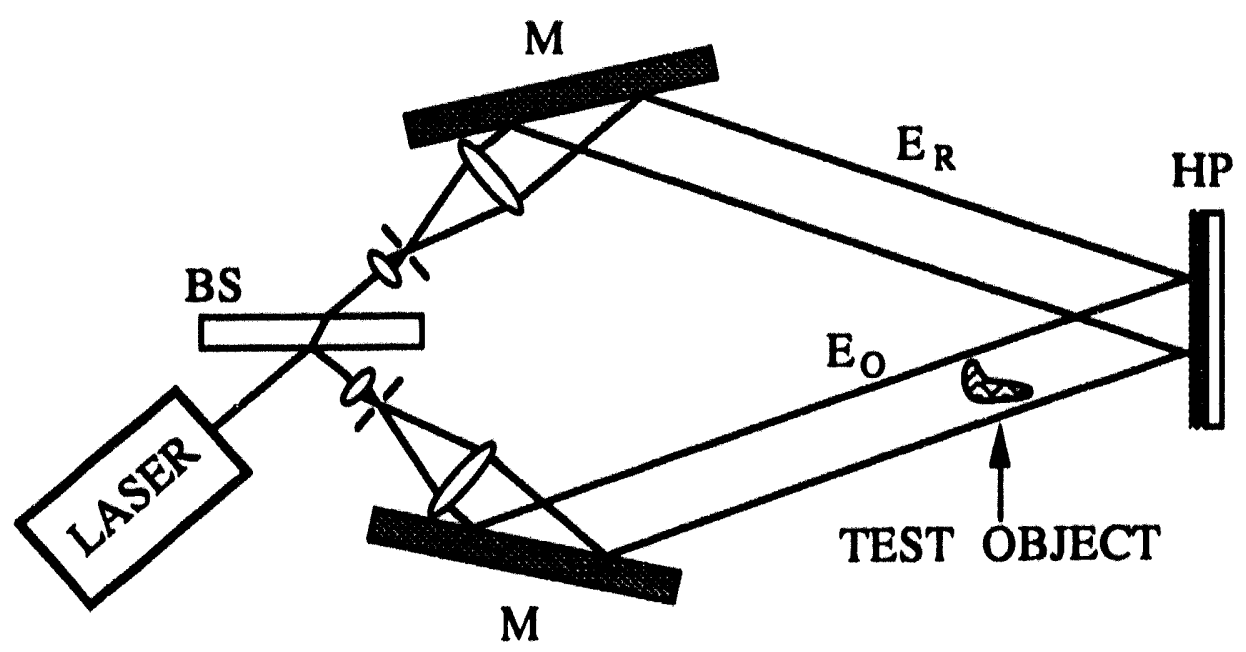

Figure 2.1 Schematic For Recording The Hologram. BS=Beam Splitter, M=Mirror, and HP=Holographic Plate.

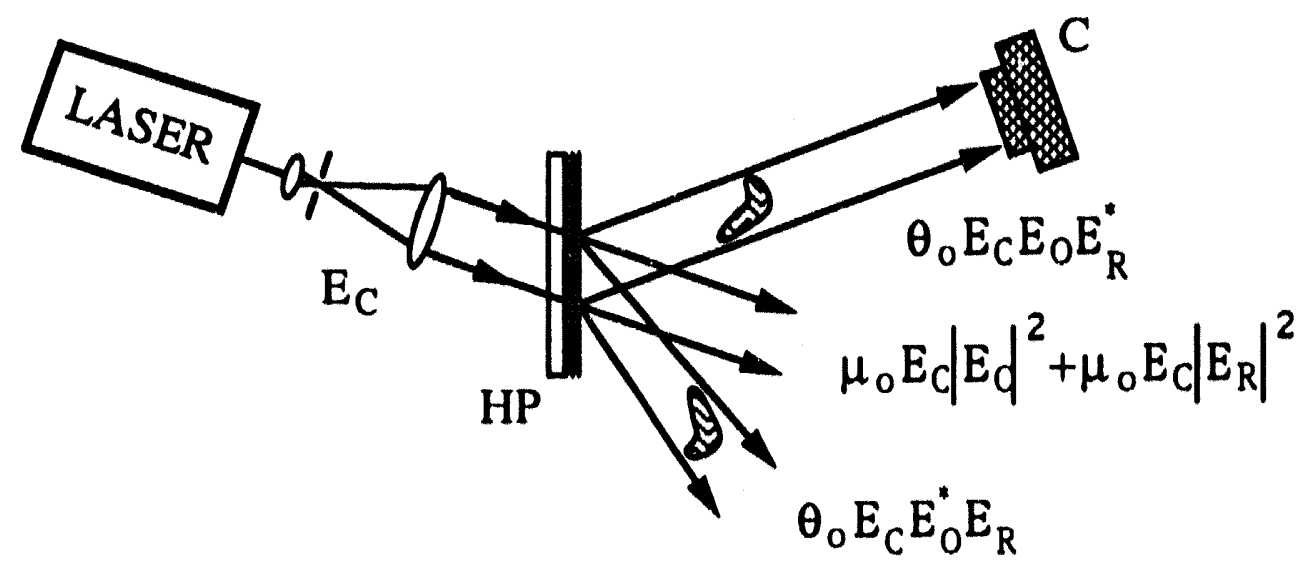

Figure 2.2 Schematic For Reconstructing The Hologram. $\mathrm{HP}=$ Holographic Plate, $\mathrm{C}=$ Camera. 
parts by a beam splitter. Both beams are expanded with a pair of convex lenses and cleaned up with an iris at the focal point. One beam passes through the object of interest before both beams are incident upon the photographic emulsion of a holographic plate. The laser is then pulsed a second time without the test object present. The holographic plate is developed appropriately and then illuminated only by the reference beam, as shown in Figure 2.2. The laser-exposed-and-then-developed portion of the holographic plate forms a diffraction pattern that causes three images to emerge from the holographic plate. The undeflected beam represents the zeroeth order wave and it contains no phase information about the test object. The other two beams are the first order deflections of the holographic diffraction pattern which contain both phase and amplitude information concerning the test object. One of the beams is the image of the test object and the other beam is its conjugate image.

Mathematically, the electric field of the laser beam can be written

$$
E\left(x, y, z, \omega, t_{L}\right)=S\left(x, y, t_{L}\right) \exp [i \omega t-i \Phi(x, y, z, \omega)],
$$

where $(x, y)$ are the spatial coordinates on the holographic plate, $(z)$ is the direction of propagation of the laser beam, the term $S\left(x, y, t_{L}\right)$ includes the temporal and spatial pulse shape of the laser, and where (dropping the $(x, y)$ notation)

$$
\begin{aligned}
\Phi(\omega, z) & =\frac{\omega}{c} \int_{0}^{z} \tilde{n}\left(\omega, z^{\prime}\right) d z^{\prime}=\frac{\omega z}{c}+\frac{\omega}{c} \int_{0}^{z}\left[\tilde{n}\left(\omega, z^{\prime}\right)-1\right] d z^{\prime} \\
& =\frac{\omega z}{c}+\phi(\omega, z)-i K(\omega, z)
\end{aligned}
$$

where $\phi$ and $K$ are real, and thus

$$
\underline{E}=S \exp \left[i \omega\left(t-\frac{z}{c}\right)-i \phi-K\right] \text {. }
$$


The intensity of light incident onto the holographic plate during a single laser pulse is

$$
\begin{aligned}
I=\left|\underline{E}_{O}+\underline{E}_{R}\right|^{2} & =S_{O}^{2} \exp \left(-2 K_{O}\right)+S_{R}^{2} \exp \left(-2 K_{R}\right) \\
& +S_{O} S_{R} \exp \left(-K_{O}-K_{R}-i \phi_{R}+i \phi_{O}\right) \\
& +S_{O} S_{R} \exp \left(-K_{O}-K_{R}+i \phi_{R}-i \phi_{O}\right) \\
& =S_{O}^{2} \exp \left(-2 K_{O}\right)+S_{R}^{2} \exp \left(-2 K_{R}\right) \\
& +2 S_{O} S_{R} \exp \left(-K_{O}-K_{R}\right) \cos \left(\phi_{R}-\phi_{O}\right),
\end{aligned}
$$

where the subscripts $O$ and $R$ stand for the object and reference beams, respectively. When this single-pulsed hologram is reconstructed, the transmission of light immediately on the right side of the holographic plate in Figure 2.2 is

$$
\underline{t}=\mu_{0} \underline{E}_{C}\left(\left|\underline{E_{O}}\right|^{2}+\left|\underline{E_{R}}\right|^{2}\right)+\theta_{0} \underline{E_{C}} \underline{E_{O}^{*}} \underline{E_{R}}+\theta_{0} \underline{E_{C}} \underline{E_{O}} \underline{E_{R}^{*}},
$$

where $\mu_{0}$ is an amplitude transmission constant with respect to the photographic developing process for the zeroeth order term and $\theta_{0}$ is a similar photographic constant for the first order terms. A photograph of the third term in Equation 2.6 does not record any information about the phase of the test object, since the laser was only pulsed once. When the dye laser is pulsed twice, once with the test object present and once without, the two reconstructions of the test object propagate collinearly and the irradiance on the film of the camera in Fig. 2.2 is

$$
I_{c}=\left|\theta_{0} \underline{E}_{C}\left(\underline{E}_{01} E_{R 1}^{*}+\underline{E}_{02} E_{R 2}^{*}\right)\right|^{2} \text {, }
$$

which gives 


$$
\begin{aligned}
I_{C}=\theta_{o}^{2}\left|E_{C}\right|^{2} S_{O}^{2} S_{R}^{2}\left\{\exp \left[-2 K_{R 1}-2 K_{O 1}\right]+\exp \left[-2 K_{R 2}-2 K_{O 2}\right]\right. \\
\left.+2 \exp \left[-K_{R 1}-K_{O 1}-K_{R 2}-K_{02}\right] \cos \left(\phi_{O 1}-\phi_{O 2}-\phi_{R 1}+\phi_{R 2}\right)\right\} .
\end{aligned}
$$

If the phase front of the reference beam is fairly constant over the width of the expanded laser beam and if absorption is minimal, the $\cos \left(\phi_{O 1}-\phi_{O 2}-\phi_{R_{1}}+\phi_{R 2}\right)$ term dominates the black and white pattern on the film. Also, any imperfections in the system optics are canceled out in the cosine term. If the mirror in the reference beam is turned slightly in between laser pulses, straight fringes appear on the reconstruction and any deviation from straight is then due to the test object. The amount of deviation is used to determine the absolute-line-integrated-density of the gas species being investigated.

\section{Resonant Holographic Interferometry}

For wavelengths very close to a resonant atomic or molecular transition of a component gas species, the real part of the index of refraction is written as (Gri64, Dre75, Ost75, Ost85)

$$
\operatorname{Re}[\tilde{n}(\omega, z)-1]=\frac{e^{2} N_{i}(z) f_{0}}{4 m \omega_{0} \varepsilon_{0}}\left[\frac{\omega_{0}-\omega}{\left(\omega-\omega_{0}\right)^{2}+\frac{\gamma^{2}}{4}}\right]
$$

where $\mathrm{N}_{\mathrm{i}}(\mathrm{z})$ is the resonant particle density, $\mathrm{f}_{0}$ is the oscillator strength of the transition with resonant frequency $\omega_{0}$, and $\gamma$ is the dissipative damping constant. This allows the total resonant phase shift to be calculated using the Equation

$$
\left(\frac{\Delta s}{s}\right)=\frac{\Delta \phi_{\text {resonant }}}{2 \pi}=\frac{\omega_{0}}{2 \pi c} \int_{0}^{z} \operatorname{Re}\left[\tilde{n}\left(\omega, z^{\prime}\right)-1\right] d z^{\prime}
$$


Equation 2.9 assumes $\left|\omega-\omega_{0}\right|$ is at least several times larger than $\gamma / 2$ and that Doppler broadening effects can be ignored. For $\left|\omega-\omega_{0}\right| » \gamma / 2$, Equation 2.9 can be written as (Dre71, Dre73, Bac80, Bow85, Rub91)

$$
\operatorname{Re}[\tilde{n}(\omega, z)-1]=\frac{e^{2} N_{i}(z) f_{0}}{4 m \omega_{0} \varepsilon_{0}}\left[\frac{1}{\omega_{0}-\omega}\right],
$$

which is called Sellmeier's Equation.

Equation 2.9 can be derived in one of two ways. The first method begins with the Kramers-Kronig Equations (Gri64, Koo78, Cra86, Mos88)

$$
\operatorname{Re}[\tilde{\mathrm{n}}-1]=\frac{1}{\pi} \operatorname{PV} \int_{-\infty}^{\infty} \frac{\operatorname{Im}[\tilde{\mathrm{n}}-1]}{\omega^{\prime}-\omega} \mathrm{d} \omega^{\prime}
$$

and

$$
\operatorname{Im}[\tilde{\mathrm{n}}-1]=\frac{1}{\pi} \operatorname{PV} \int_{-\infty}^{\infty} \frac{\operatorname{Re}[\tilde{\mathrm{n}}-1]}{\omega^{\prime}-\omega} \mathrm{d} \omega^{\prime}
$$

where PV stands for Cauchy's principle value, and then assumes that

$$
\operatorname{Im}[\tilde{n}-1] \equiv \frac{e^{2} N f_{0}}{4 m \omega_{0} \varepsilon_{0}}\left[\frac{\gamma / 2}{\left(\omega-\omega_{0}\right)^{2}+\frac{\gamma^{2}}{4}}\right] .
$$

Evaluating Equation 2.12 with Equation 2.14 then gives Equation 2.9. Together, n-1 can then be written (Gri64)

$$
\tilde{n}-1=\frac{e^{2} N_{i} f_{0}}{4 m \omega_{0} \varepsilon_{0}}\left[\frac{-1}{\omega-\omega_{0}+i \frac{\gamma}{2}}\right] \text {. }
$$

The second method to derive Equation 2.9 is to assume that the transparent test section can be treated as a classical dielectric medium, thus giving (Mar67, Mea70, Ber71, Sie77, Koo79, Ode80, Jel81) 


$$
\frac{\tilde{n}^{2}-1}{\tilde{n}^{2}+2}=\frac{1}{3 \varepsilon_{0}} \sum_{1} \tilde{\alpha}_{i} N_{i}
$$

and then use the quantum mechanical value of the complex polarizability

$$
\tilde{\alpha}_{i}=\frac{\left(e^{2} / m\right) f_{i k}}{\omega_{i k}^{2}-\omega^{2}+i \gamma_{k j} \omega} .
$$

Equation 2.9 (a3 well as Equation 2.15) follows when assuming that only one resonance transition is significant. For a more detailed derivation of the second method, see Appendix 1.

When $\left|\omega-\omega_{0}\right|-\gamma / 2$ and when Doppler broadening is significant in comparison to natural line broadening (and other broadening mechanisms), Equation 2.9 is modified by using the Doppler shifted frequency (Mar67, Mea70, Jel81, Kug83, Cra86, Bon86, Woi91, Woi92)

$$
\omega^{\prime} \equiv \omega\left(1+\frac{v_{x}}{c}\right),
$$

where $v_{x}$ is the velocity component in the direction of the laser beam, and by using a Maxwell-Boltzmann distribution of velocities for the gaseous test section

$$
N_{i}^{\prime}=N_{i}\left(\frac{m}{2 \pi k_{B} T}\right)^{1 / 2} \int_{-\infty}^{\infty} d v_{x} \exp \left(\frac{-m v_{x}^{2}}{2 k_{B} T}\right) .
$$

Using Measures' notation, this leads to (see Appendix 1)

$$
\tilde{n}-1 \cong\left\{\frac{c}{\omega_{0}}\right\}(-i A) N_{i} W(-u+i \alpha)
$$

or when line-integrating along the path of the laser beam

$$
\phi-i k=-i A \overline{N_{i} L} W(-u+i \alpha) \text {, }
$$

where 


$$
\beta=\frac{\omega_{0}}{c} \sqrt{\frac{2 k_{B} T}{m}} \quad, \quad \alpha=\frac{\gamma_{i k}}{2 \beta}, \quad u=\frac{\omega-\omega_{0}}{\beta}, \quad A=\left(\frac{\pi^{3 / 2} r_{0} c f_{i k}}{\beta}\right) \text {, }
$$

$\overline{N_{1} \mathrm{~L}}$ is the line-integrated-density, $r_{0}=e^{2} /\left(4 \pi \varepsilon_{0} \mathrm{mc}^{2}\right)=2.818 \times 10^{-13} \mathrm{~cm}$ is the classical electron radius and

$$
W(z)=\exp \left(-z^{2}\right) x(1+\operatorname{erf}\{i z\})
$$

where the real and imaginary parts of $W(z)$ for $\operatorname{Im}[z]=0$ are shown in Figure 2.3. Noting that for $|z| \gg 2$ (Abr72)

$$
W(z) \rightarrow \frac{i z}{\sqrt{\pi}|z|^{2}},
$$

equation 2.15 is recovered.

It is notable that the method for accounting for Doppler broadening used above is also called the Voigt profile method since it begins with a

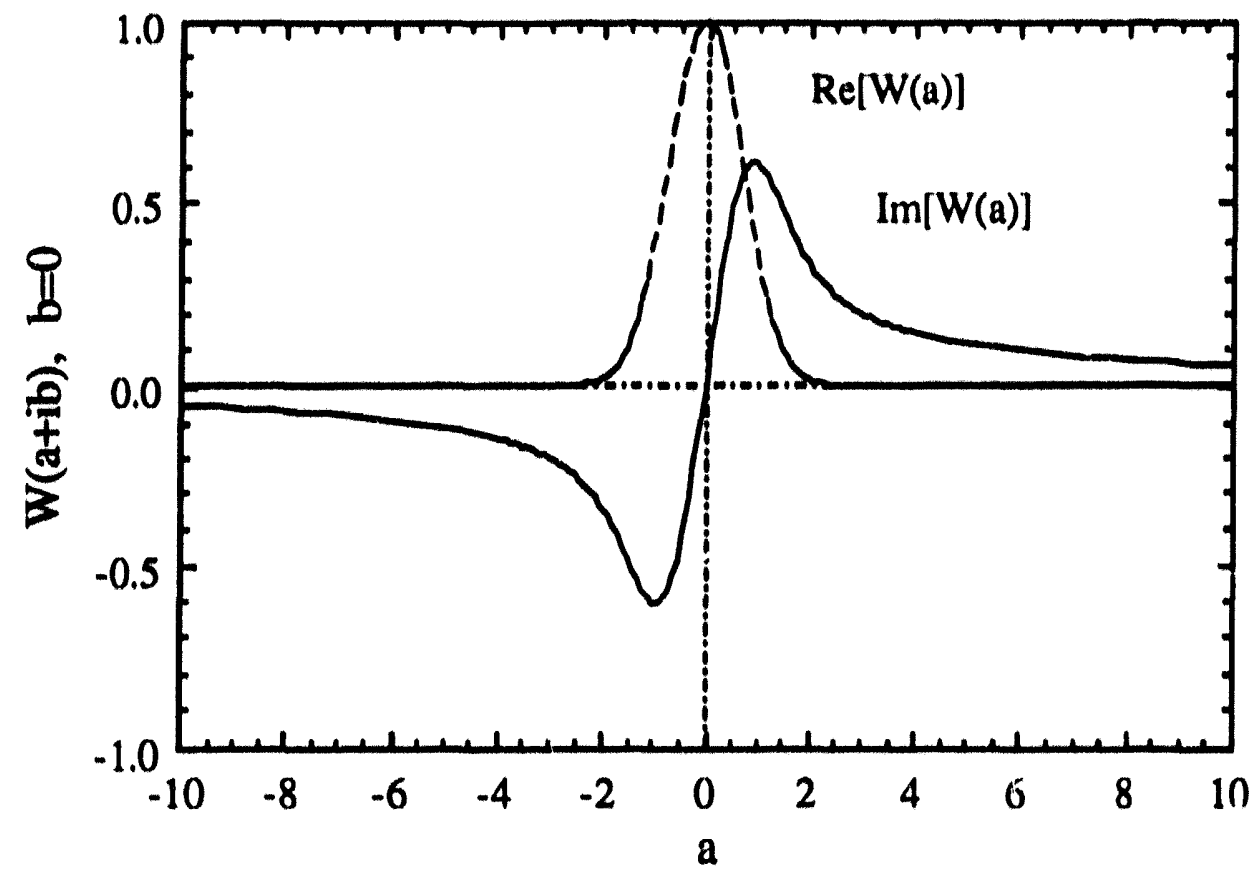

Fig. 2.3 The basic response curve for $W(z)$, and thus for resonant interferometry when accounting for Doppler Shift. 
Lorentzian-shaped natural broadening, Equation 2.15, and convolutes it with a Gaussian-shaped velocity distribution/line broadening mechanism, Equation 2.19.

Another notable item is the polarity of $\operatorname{Im}[\tilde{n}-1]$ since there is some discrepancy in the open literature on this point. In Chapter 4, experimental e idence is presented to agree with (Gri64, Mar67, Mea70, Dre71, Ber71, Dre73, Dre75, Ost75, Sie77, Koo79, Koo78, Jel81, Ost85, Bow85, Cra86, Mos88, Sta88) that

$$
\operatorname{sgn}\{\operatorname{Im}[\tilde{\mathbf{n}}-1]\}=-\operatorname{sgn}\left\{\omega-\omega_{0}\right\}
$$

is correct (as in Fig. 2.3), where $\operatorname{sgn}\{x\}$ is the function

$$
\operatorname{sgn}(x)=\left\{\begin{array}{rl}
1 & x>0 \\
0 & x=0 \\
-1 & x<0
\end{array}\right\}
$$

as opposed to the polarity

$$
\operatorname{sgn}\{\operatorname{Im}[\tilde{\mathbf{n}}-1]\}=+\operatorname{sgn}\left\{\omega-\omega_{0}\right\}
$$

given by (Küg83, Sei86, Woi91, Woi92). In addition, Odenthal, et al. (Ode80) and Rubin, et al. (Rub91) used Equations following Eqn. 2.25 but presented graphs following Eqn. 2.27.

\section{Accounting For Finite Laser Bandwidth}

Dreiden, et al. (Dre75) accounted for finite laser bandwidth by ignoring Doppler broadening (and thus using Eqn. 2.9), assuming that the line-integrated-density of the test section was near the lower detectability limit, and by doing a simple average of the calculated fringe shift over the FWHM of the laser line, 


$$
\begin{aligned}
& \overline{\left(\frac{\Delta s}{s}\right)}=\frac{\overline{\mathrm{NL}} \mathrm{r}_{\mathrm{o}} \mathrm{cf}}{2 \delta \omega} \int_{\omega-(\delta \omega / 2)}^{\omega+(\delta \omega / 2)} \mathrm{d} \omega_{1} \frac{\omega_{0}-\omega_{1}}{\left(\omega_{1}-\omega_{0}\right)^{2}+(\gamma / 2)^{2}} \\
& =\frac{\overline{\mathrm{NL}} r_{0} c f_{0}}{2 \delta \omega} \frac{1}{2} \ln \left(\frac{1+\mathrm{x}}{1-\mathrm{x}}\right)=\frac{\overline{\mathrm{NL}} \mathrm{r}_{0} \mathrm{cf} \mathrm{o}_{0}}{2 \delta \omega} \operatorname{arctanh}(\mathrm{x})
\end{aligned}
$$

where

$$
x=\frac{\left(\omega_{0}-\omega_{1}\right) \delta \omega}{\left(\omega_{1}-\omega_{0}\right)^{2}+(\gamma / 2)^{2}+(\delta \omega / 2)^{2}}
$$

and $\delta \omega$ is the FWHM bandwidth of the laser intensity. With this analysis, Dreiden determined that an increase in laser bandwidth causes a decrease in the sensitivity of the diagnostic and a contraction of the range of measurable line-densities using resonant interferometry techniques.

To more fully account for the interaction between the Gaussian shape of the laser bandwidth used in this work and the Voigt profile obtained for the region $\left|\omega-\omega_{0}\right| \geq \approx(\gamma / 2)$ when accounting for Doppler broadening, and to account for the interference effects induced at the holographic plate by a finite bandwidth light source, the temporalcoherence discussions of Goodman for interferometry are utilized (Goo85). Here, the laser is simplistically modeled as producing one photon at a time with a random frequency, $\omega$, which has a Gaussian probability distribution about a center frequency, $\varpi$. It is then sufficient to time-average the random frequency of the laser. By convention, this is written

$$
\underline{\Gamma}(\tau)=\left\langle\underline{E}(t+\tau) \underline{E}^{*}(t)\right\rangle=\lim _{T \rightarrow \infty} \frac{1}{T} \int_{-T / 2}^{+T / 2} \underline{E}(t+\tau) \underline{E}^{*}(t) d t,
$$

where $\underline{\Gamma}(\tau)$ is the complex autocorrelation function, $\underline{E}(t)$ is the complex electric field of the laser, and $<>$ denotes a time average. The electric 
field of the laser is considered ergodic when the time average of $\underline{E}(t+\tau) \underline{E}^{*}(t)$ is equal to the probability average of $\underline{E}(t+\tau) \underline{E}^{*}(t)$; i.e., when

$$
\left\langle\underline{E}(t+\tau) \underline{E}^{*}(t)\right\rangle=\bar{E}(t+\tau) \underline{E}^{*}(t),
$$

where

$$
\overline{\underline{E}(t+\tau) \underline{E}^{*}(t)}=\iint_{-\infty}^{\infty} \underline{E}(t+\tau) \underline{E}^{*}(t) p\left(\underline{E}(t+\tau), \underline{E}^{*}(t) ; t, t+\tau\right) d \underline{E} d \underline{E}^{*}
$$

and $p\left(\underline{E}(t+\tau), \underline{E}^{*}(t) ; t, t+\tau\right)$ is the probability distribution of the electric fields. If the electric field of the laser is ergodic, then the WienerKhinchin Theorem can be used, which states

$$
\Gamma(\tau)=\int_{-\infty}^{\infty} G(\omega) \exp (-i \omega \tau) d \omega
$$

where $G(\omega)$ is the laser frequency probability

$$
G(\omega)=\lim _{T \rightarrow \infty} \frac{\overline{\varepsilon(\omega) \underline{\varepsilon}^{*}(\omega)}}{T}
$$

and $\underline{\varepsilon}(\omega)$ is the Fourier transform of the complex electric field

$$
\underline{\varepsilon}(\omega)=\int_{-\infty}^{\infty} \underline{E}(t) \exp (-i \omega t) d t
$$

Given

$$
\underline{\Gamma}(\tau=0)=\int_{-\infty}^{\infty} G(\omega) d \omega=s^{2}
$$

and

$$
\hat{\mathbf{G}}(\omega)=\frac{\mathrm{G}(\omega)}{\mathrm{S}^{2}},
$$

the laser frequency probability with a Gaussian frequency distribution is then written as 


$$
\hat{G}(\omega) \cong \frac{2 \sqrt{\ln 2}}{\sigma \sqrt{\pi}} \exp \left[-\left(2 \sqrt{\ln 2} \frac{\omega-\varpi}{\sigma}\right)^{2}\right],
$$

where $\sigma$ is the Full Width at Half Maximum (FWHM) of the laser intensity. This means that the time average of the electric fields on the holographic plate can be written as

$$
\Gamma(\tau)=\frac{2 S^{2} \sqrt{\ln 2}}{\sigma \sqrt{\pi}} \int_{-\infty}^{\infty} \exp \left[-4 \ln 2\left(\frac{\omega-\Phi}{\sigma}\right)^{2}-i \omega \tau\right] d \omega
$$

Note that Eqn. 2.39 only includes temporal coherence (two beams delayed in time) and not spatial coherence (two beams shifted in space). For this work, it is assumed that the spatial coherence of the laser is sufficient so that the relevant mathematics may be neglected.

Equation 2.4 can now be rewritten

$$
\langle\mathrm{I}\rangle=\Gamma_{\mathrm{OO}}+\Gamma_{\mathrm{RR}}+\Gamma_{\mathrm{OR}}+\Gamma_{\mathrm{OR}}^{*}
$$

and Eqn. 2.7 can be rewritten as

$$
\left\langle I_{c}\right\rangle=\left|\theta_{0} E_{c}\left(\Gamma_{O R 1}+\Gamma_{O R 2}\right)\right|^{2}
$$

where "1" and "2" refer to the first and second dye laser pulses, respectively.

\section{The Solution for Doppler Broadening and Finite Bandwidth}

Recalling the notation for the electric fields given in Equations 2.12.3, the two laser beams for both laser pulses can be written

$$
\Phi_{O 1}=\frac{\omega z}{c}+\omega \tau_{O}-i K_{O}
$$




$$
\begin{aligned}
& \Phi_{R 1}=\frac{\omega z}{c}+\omega \tau_{R}-i K_{R}, \\
& \Phi_{O 2}=\frac{\omega z}{c}+\omega \tau_{O}-i K_{O}-i A \overline{N_{i} L} W(-u+i \alpha),
\end{aligned}
$$

and

$$
\Phi_{\mathrm{R} 2}=\frac{\omega \mathrm{z}}{\mathrm{c}}+\omega \tau_{\mathrm{R}}-\mathrm{iK_{ \textrm {R } }}+\omega \mathrm{y} \delta \text {, }
$$

where $\mathrm{K}_{\mathrm{O}}$ and $\mathrm{K}_{\mathrm{R}}$ represent any absorption incurred outside the test section by the object and reference beams, respectively, and to and $t_{R}$ refer to the travel-time of the object and reference beams, respectively, as they travel between the incident side of the $f$ and the holographic plate (see Fig. 2.1).

The Doppler broadened test section $\left(-i \mathrm{~A} \overline{N_{i} L} W(-u+i \alpha)\right)$ is now included in $\Phi_{\mathrm{O} 2}$ and the reference fringes caused by turning a mirror in the reference beam between laser pulses, $\omega y \delta$, is accounted for in $\Phi_{R 2}$. This notation for reference fringes means that the black and white stripes are in the $\hat{x}$ direction with the variation in the $\hat{y}$ direction whose spacing is determined by the constant $\delta$. Recall also that each of these variables is a function of $(x, y)$, the spatial coordinates on the holographic plate; their notation is omitted here for simplicity.

In anticipation of using Eqns. 2.42-2.45 in Eqn. 2.39, one writes

$$
\begin{aligned}
& i \omega \tau_{\mathrm{OO1}}=\mathrm{i}\left(\Phi_{\mathrm{O} 1}-\Phi_{\mathrm{O} 1}^{*}\right)=2 \mathrm{~K}_{\mathrm{O}}, \\
& \mathrm{i} \omega \tau_{\mathrm{RR} 1}=\mathrm{i}\left(\Phi_{\mathrm{R} 1}-\Phi_{\mathrm{R} 1}^{*}\right)=2 \mathrm{~K}_{\mathrm{R}}, \\
& \mathrm{i} \omega \tau_{\mathrm{OR} 1}=\mathrm{i}\left(\Phi_{\mathrm{O} 1}-\Phi_{\mathrm{R} 1}^{*}\right)=\mathrm{i} \omega\left(\tau_{\mathrm{O}}-\tau_{\mathrm{R}}\right)+\mathrm{K}_{\mathrm{O}}+\mathrm{K}_{\mathrm{R}}, \\
& \mathrm{i} \omega \tau_{\mathrm{O} 2}=\mathrm{i}\left(\Phi_{\mathrm{O} 2}-\Phi_{\mathrm{O} 2}^{*}\right)
\end{aligned}
$$




$$
=2 \mathrm{~K}_{\mathrm{o}}+\mathrm{A} \overline{\mathrm{N}_{\mathrm{i}} \mathrm{L}}[\mathrm{W}(-\mathrm{u}+\mathrm{i} \alpha)+\mathrm{W}(+\mathrm{u}+\mathrm{i} \alpha)]
$$

(utilizing the relation (Abr72)

$$
\begin{aligned}
& {[W(z)]^{*}=W\left(-[z]^{*}\right),,} \\
& i \omega \tau_{R R 2}=i\left(\Phi_{R 2}-\Phi_{R 2}^{*}\right)=2 K_{R},
\end{aligned}
$$

and

$$
\begin{aligned}
& \mathrm{i} \omega \tau_{\mathrm{OR} 2}=\mathrm{i}\left(\Phi_{\mathrm{O} 2}-\Phi_{\mathrm{R} 2}^{*}\right) \\
& =\mathrm{i} \omega\left(\tau_{\mathrm{O}}-\tau_{\mathrm{R}}-\delta \mathrm{y}\right)+\mathrm{K}_{\mathrm{O}}+\mathrm{K}_{\mathrm{R}}+\mathrm{A} \overline{\mathrm{N}_{\mathrm{i}} \mathrm{L}} \mathrm{W}(-\mathrm{u}+\mathrm{i} \alpha) .
\end{aligned}
$$

Now inserting Equations 2.46, 2.47, 2.48, and 2.51 into Equation 2.39, one obtains

$$
\begin{aligned}
& \Gamma_{O O 1}=\Gamma\left(\tau_{O O 1}\right)=S_{O}^{2} \exp \left[-2 K_{O}\right], \\
& \Gamma_{R R 1}=S_{R}^{2} \exp \left[-2 K_{R}\right], \\
& \Gamma_{O R 1}=S_{O} S_{R} \exp \left[-K_{O}-K_{R}-\frac{\sigma^{2}\left(\tau_{O}-\tau_{R}\right)^{2}}{16 \ln 2}-i \varpi\left(\tau_{O}-\tau_{R}\right)\right],
\end{aligned}
$$

and

$$
\Gamma_{\mathrm{RR} 2}=\mathrm{S}_{\mathrm{R}}^{2} \exp \left[-2 \mathrm{~K}_{\mathrm{R}}\right] \text {. }
$$

The $\sigma^{2}\left(\tau_{O}-\tau_{R}\right)^{2} /(16 \ln 2)$ term in Equation 2.55 is the temporal coherence term. If the time-travel difference between the object and reference beams becomes much larger than the coherence-time of the laser, $(2 \pi / \sigma)$, then $\Gamma_{\mathrm{OR} 1} \rightarrow 0$ and no fringes are observed at all.

$\Gamma_{\mathrm{OO2}}$ and $\Gamma_{\mathrm{OR} 2}$ are not analytically solvable using Equation 2.39 because of the presence of $W(z)$ in the exponential, so their solutions are approximated in this work using the Saddle Point Theorem (Arf85), where

$$
\int_{-\infty}^{\infty} d x \exp [f(x)] \cong \int_{-\infty}^{\infty} d x \exp \left[f\left(x_{s}\right)+\frac{1}{2} f^{\prime \prime}\left(x_{s}\right)\left\{x-x_{s}\right\}^{2}\right]
$$




$$
\cong \eta \exp \left[\mathrm{f}\left(\mathrm{x}_{\mathbf{s}}\right)\right] \sqrt{\frac{1}{\mathrm{f}^{\prime \prime}\left(\mathrm{x}_{\mathbf{8}}\right)}}
$$

where $\eta$ is a constant and $x_{8}$ is a solution of $f^{\prime}\left(x_{8}\right)=0$.

Inserting Equation 2.49 into Equation 2.39 gives

$$
\begin{array}{r}
\Gamma_{\infty 22}=\frac{2 S_{0}^{2} \sqrt{\ln 2}}{\sigma \sqrt{\pi}} \int_{-\infty}^{\infty} d \omega \exp \left[-4 \ln 2\left(\frac{\omega-\Phi}{\sigma}\right)^{2}-2 K_{O}\right. \\
\left.-A \overline{N_{i} L}[W(-u+i \alpha)+W(+u+i \alpha)]\right] .
\end{array}
$$

Letting $\omega^{\prime}=\omega-\bar{\sigma}, \Delta=\left(\Phi-\omega_{0}\right) / \beta, \Lambda=\omega^{\prime} / \beta$, and $u=\Delta+\Lambda$, Equation 2.58 becomes

$$
\Gamma_{\infty 22}=\frac{2 S_{0}^{2} \beta \sqrt{\ln 2}}{\sigma \sqrt{\pi}} \int_{-\infty}^{\infty} \mathrm{d} \Lambda \exp [\mathrm{h}(\Lambda)]
$$

where

$$
h(\Lambda)=-4 \ln 2\left(\frac{\beta}{\sigma}\right)^{2} \Lambda^{2}-2 K_{0}-A \overline{N_{i} L}[W(\psi)+W(\chi)],
$$

$\psi=-\Delta-\Lambda+i \alpha$, and $\chi=+\Delta+\Lambda+i \alpha$. (Note that $\operatorname{Im}[W(\psi)+W(\chi)]=0$ ) This in turn leads to

$$
h^{\prime}(\Lambda)=-8 \ln 2\left(\frac{\beta}{\sigma}\right)^{2} \Lambda-2 A \overline{N_{i} L}[\psi W(\psi)+\chi W(\chi)]
$$

and

$$
\begin{aligned}
h^{\prime \prime}(\Lambda)= & -8 \ln 2\left(\frac{\beta}{\sigma}\right)^{2}-2 A \overline{N_{i} L}\left\{W(\psi)\left[2 \psi^{2}-1\right],\right. \\
& \left.-W(\chi)\left[2 \chi^{2}-1\right]-\frac{4 i}{\sqrt{\pi}}(\Delta+\Lambda)\right\},
\end{aligned}
$$

where Equation 2.61 is solved for the complex solution $\Lambda_{s}$ from $h^{\prime}\left(\Lambda_{s}\right) \equiv 0$ using Newton's Method in Mathematica (see Appendix 2). Note that as 
$\sigma \rightarrow 0$ in Eqn. 2.62 and thus $\Lambda_{3} \rightarrow 0$ in the solution to $h^{\prime}\left(\underline{\Lambda_{3}}\right)=0$, Eqn. 2.58 becomes solvable analytically, giving

$$
\Gamma_{\infty 02} \rightarrow s_{0}^{2} \exp \left[-2 K_{0}-A \overline{N_{i} L}\{W(\psi)+W(\chi)\}\right] .
$$

Therefore for small $\Lambda_{b}$,

$$
\eta_{h}=\sqrt{\frac{\pi}{4 \ln 2}}\left(\frac{\sigma}{\beta}\right) \sqrt{h^{\prime \prime}(0)}
$$

or

$$
\Sigma_{\mathrm{OO} 2} \cong S_{0}^{-} \sqrt{\frac{h^{\prime \prime}(0)}{h^{\prime \prime}\left(\Lambda_{s}\right)}} \exp \left[h\left(\Lambda_{s}\right)\right]
$$

Although $h^{\prime \prime}(\Lambda)$ is complex-valued, it was numerically determined here that $\left(h^{\prime \prime}(0) / h^{\prime \prime}(\Lambda)\right) \sim\left|h^{\prime \prime}(0) / h^{\prime \prime}(\Lambda)\right|$ and thus justifying the use of the absolute value in Eqn. 2.65 .

Finally, Equation 2.52 and Equation 2.39 combine to make

$$
\begin{gathered}
\Gamma_{\mathrm{OR} 2}=\frac{2 S_{O} S_{R} \beta \sqrt{\ln 2}}{\sigma \sqrt{\pi}} \int_{-\infty}^{\infty} \mathrm{d} \omega \exp \left[-4 \ln 2\left(\frac{\omega-\sigma}{\sigma}\right)^{2}-K_{O}-K_{R}\right. \\
\left.-i \omega\left(\tau_{O}-\tau_{R}-\delta y\right)-A \overline{N_{i} L} W(-u+i \alpha)\right] .
\end{gathered}
$$

Letting $\omega^{\prime}=\omega-\bar{\varpi}, \Delta=\left(\varpi-\omega_{0}\right) / \beta, \varepsilon=\omega^{\prime} / \beta, u=\Delta+\varepsilon$, and $\Theta=-\Delta-\varepsilon+i \alpha$, Equation 2.66 becomes

$$
\underline{\Gamma}_{\mathrm{OR} 2}=\frac{2 S_{\mathrm{O}} S_{R} \beta \sqrt{\ln 2}}{\sigma \sqrt{\pi}} \int_{-\infty}^{\infty} \mathrm{d} \varepsilon \exp [g(\varepsilon)]
$$

where

$$
\begin{aligned}
& g(\varepsilon)=-4 \ln 2\left(\frac{\beta}{\sigma}\right)^{2} \varepsilon^{2}-K_{O}-K_{R}-i \sigma\left(\tau_{O}-\tau_{R}-\delta y\right)-A \overline{N_{i} L} W(\Theta) \\
& g^{\prime}(\varepsilon)=-8 \ln 2\left(\frac{\beta}{\sigma}\right)^{2} \varepsilon-2 A \overline{N_{i} L} \Theta W(\Theta)+i\left(\frac{2}{\sqrt{\pi}}\right) A \overline{N_{i} L}
\end{aligned}
$$


and

$$
g^{\prime \prime}(\varepsilon)=-8 \ln 2\left(\frac{\beta}{\sigma}\right)^{2}-2 A \overline{N_{i} L} W(\Theta)\left\{2 \Theta^{2}-1\right\}+i\left(\frac{4}{\sqrt{\pi}}\right) A \overline{N_{i} L} \theta
$$

The $\omega^{\prime}\left(\tau_{0}-\tau_{R}-\delta y\right)$ term has been dropped to make the integration possible, thereby assuming $\omega^{\prime}$ « $\omega$ for the values of $\omega^{\prime}$ where the $4 \ln 2(\omega / \sigma)^{2}$ term does not dominate in the exponential of Eqn. 2.66. Equation 2.69 is solved for the complex solution $\varepsilon_{g}$ from $g^{\prime}\left(\varepsilon_{g}\right)=0$ by rearranging it to the form

$$
W\left(-\Delta-\varepsilon_{s}+i \alpha\right)=\frac{-\left(\frac{4 \ln 2 \beta^{2}}{A \overline{N_{i} L} \sigma^{2}}\right) \varepsilon_{s}+i\left(\frac{1}{\sqrt{\pi}}\right)}{\left(-\Delta-\varepsilon_{s}+i \alpha\right)}
$$

and then solving for $\varepsilon_{\text {a }}$ using Newton's Method in Mathematica (see Appendix 2). Again noting that as $\sigma \rightarrow 0$ in Eqn. 2.66 and thus $\varepsilon_{g} \rightarrow 0$ in the solution to $g^{\prime}(\underline{\varepsilon})=0$, Eqn. 2.66 becomes solvable analytically, giving

$$
\begin{aligned}
\Gamma_{\mathrm{OR} 2} & \rightarrow S_{O} S_{R} \exp \left[-K_{O}-K_{R}-\frac{\sigma^{2}\left(\tau_{O}-\tau_{R}-\delta y\right)^{2}}{16 \ln 2}\right. \\
& \left.-i \Phi\left(\tau_{O}-\tau_{R}-\delta y\right)-A \overline{N_{i} L} W(-\Delta+i \alpha)\right]
\end{aligned}
$$

Therefore for small $\underline{\varepsilon_{\mathbf{s}}}$,

$$
\eta_{g}=\sqrt{\frac{\pi}{4 \ln 2}}\left(\frac{\sigma}{\beta}\right) \sqrt{g^{\prime \prime}(0)} \exp \left[-\frac{\sigma^{2}\left(\tau_{O}-\tau_{R}-\delta y\right)^{2}}{16 \ln 2}\right]
$$

or

$$
\left.\underline{\Gamma}_{\mathrm{OR} 2} \cong S_{\mathrm{O}} S_{R} \sqrt{\frac{g^{\prime \prime}(0)}{g^{\prime \prime}\left(\varepsilon_{s}\right)}}\right] \exp \left[-\frac{\sigma^{2}\left(\tau_{0}-\tau_{R}-\delta y\right)^{2}}{16 \ln 2}+g\left(\varepsilon_{s}\right)\right]
$$

Two roots were found for Eqn. 2.71. One of the roots changed continuously as $\Delta$ and NL were varied, while the other root was discontinuous for the same parameter regions. Therefore the first root was used in the solutions that follow. 
Equation 2.41 gives the irradiance onto the reconstruction camera film. Assuming the temporal coherence requirement is easily met,

$$
\frac{\sigma^{2}\left(\tau_{O}-\tau_{R}-\delta y\right)^{2}}{16 \ln 2} \approx \frac{\sigma^{2}\left(\tau_{O}-\tau_{R}\right)^{2}}{16 \ln 2} \sim \text { very small, }
$$

Equation 2.41 can now be written

$$
\left\langle I_{c}\right\rangle=M \exp [-N]\{1+P+Q \cos [T]\}
$$

where

$$
\begin{aligned}
& M=\theta_{0}^{2}\left|E_{C}\right|^{2} S_{O}^{2} S_{R}^{2} \\
& N=2 K_{O}+2 K_{R}+\frac{\sigma^{2}\left(\tau_{O}-\tau_{R}\right)^{2}}{8 \ln 2} \\
& P=\Omega_{g}^{2} \exp \left[2 R\left(\varepsilon_{g}\right)\right] \\
& Q=2 \Omega_{g} \exp \left[R\left(\underline{\varepsilon_{g}}\right)\right]=2 \sqrt{P} \\
& T=\varpi y \delta+Y\left(\varepsilon_{g}\right)
\end{aligned}
$$

and where

$$
\begin{aligned}
& \Omega_{g}=\sqrt{\frac{g^{\prime \prime}(0)}{g^{\prime \prime}\left(\underline{\varepsilon_{s}}\right)} \mid} \\
& R\left(\underline{\varepsilon_{s}}\right)=\operatorname{Re}\left[-4 \ln 2\left(\frac{\beta}{\sigma}\right)^{2} \underline{\varepsilon}_{s}^{2}-A \overline{N_{i} L} W\left(-\Delta-\underline{\varepsilon_{g}}+i \alpha\right)\right] \\
& Y\left(\underline{\varepsilon_{s}}\right)=\operatorname{lm}\left[-4 \ln 2\left(\frac{\beta}{\sigma}\right)^{2} \underline{\varepsilon}_{s}^{2}-A \overline{N_{i} L} W\left(-\Delta-\underline{\varepsilon_{g}}+i \alpha\right)\right] .
\end{aligned}
$$

Since the reference fringes, reconstructed holograms are analyzed, that term may be dropped here and thus the fringe shift can be interpreted as 


$$
\left(\frac{\Delta s}{s}\right)=\frac{T}{2 \pi}=\frac{Y\left(\varepsilon_{s}\right)}{2 \pi}
$$

Notice that as $\sigma \rightarrow 0$ in Eqn. 2.66 and thus $\varepsilon_{s} \rightarrow 0$ in the solution to $g^{\prime}\left(\underline{\varepsilon_{s}}\right)=0$, Measures' answer is obtained (Mea70)

$$
\left(\frac{\Delta s}{s}\right) \rightarrow \operatorname{Im}\left[-\frac{A \overline{N_{i} L}}{2 \pi} W(-\Delta+i \alpha)\right] .
$$

The ablation plumes in this work are assumed to have a Maxwellian parallel velocity distribution (i.e., as seen by the dye laser beam) in the range $0.1 \mathrm{eV}-1.0 \mathrm{eV}$ (Dre86, Dre91). As an example of fringe shift interpretation for holographic interferometry, Measures' theory (Eqn. 2.86) is graphed in Fig. 2.4 using the temperatures $0.1 \mathrm{eV}, 0.3 \mathrm{eV}$, and $1.0 \mathrm{eV}$ for the $A l(I), 394.401 \mathrm{~nm}$, resonance line $\left(f_{0}=0.115\right)$. Although the diagnostic is most sensitive for $|\Delta \lambda|=0.0025 \mathrm{~nm}$, that is also the region of most uncertainty due to the plume temperature. However for $|\Delta \lambda|>0.010$ $\mathrm{nm}$, the fringe shift interpretation is no longer dependent upon temperature. Since the temperatures of the ablation plumes in this work were not measured, a temperature of $0.3 \mathrm{eV}$ was assumed for the interpretation of all holograms.

Figure 2.5 compares the Gaussian dye laser bandwidth to Measures' interpretation curves for four temperatures. Note that as the temperature increases, the maximum amount of observable fringe shift decreases, the wavelength of the peak fringe shift increases, and the effective bandwidth of the dye laser narrows.

Whereas line-density versus the fringe shift is a linear relationship at each $\Delta \lambda$ in Fig. 2.4 and Fig. 2.5, this is not the case for the theory developed here. Table 2.1 shows example values of the parameters used in this work, and Figure 2.6 shows a sample hologram interpretation graph at 


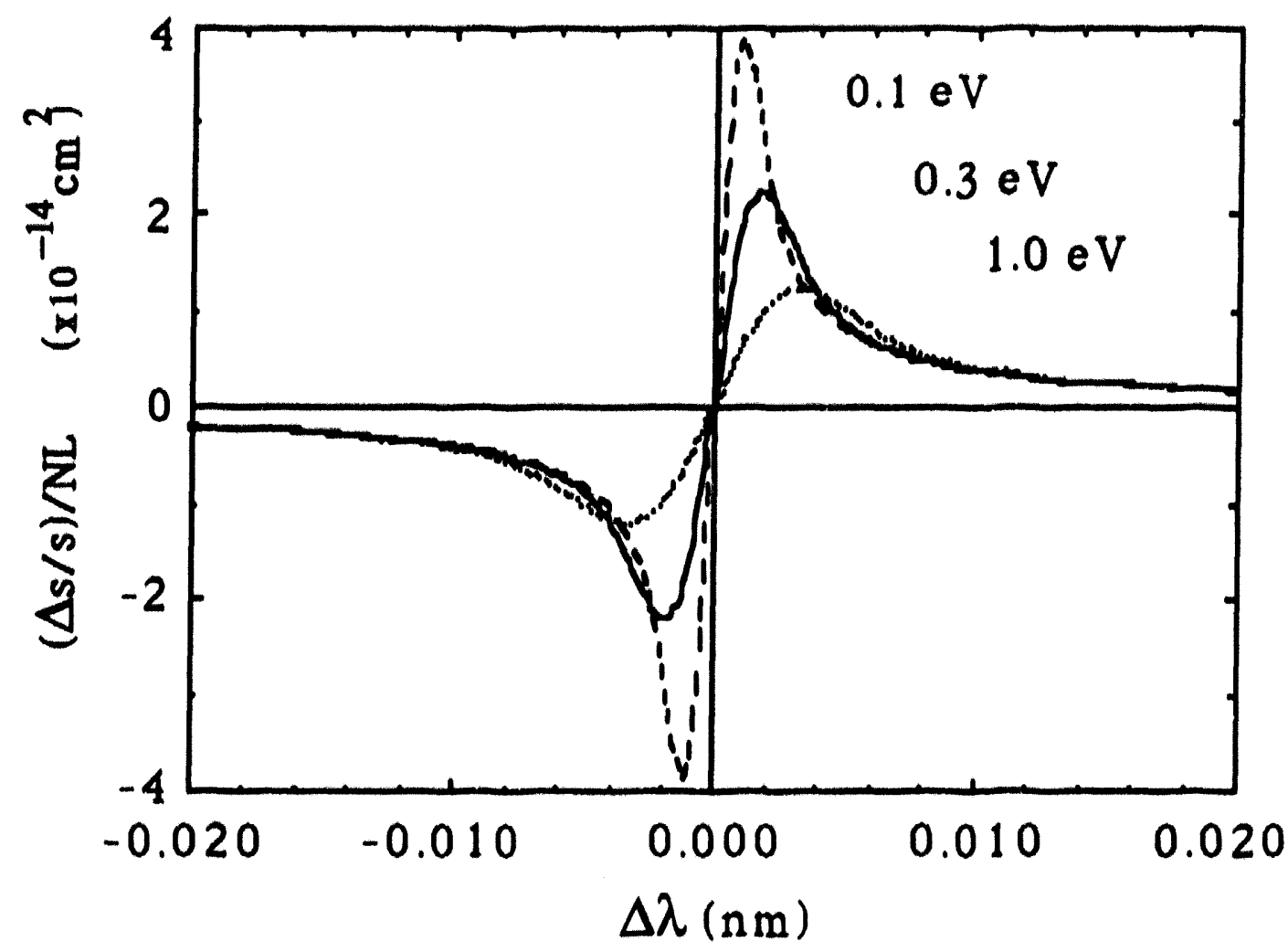

Fig. 2.4 Fringe shift per line density, $(\Delta s / s) / N L$, as a function of $\Delta \lambda=\left(\lambda-\lambda_{0}\right)$ for the neutral aluminum line $\lambda_{0}-394.401 \mathrm{~nm}$. Calculations made used Measures' theory and thus takes Doppler shift into account but assumes an infinitesimal laser bandwidth (Mea70).

$|\Delta \lambda|=0.003 \mathrm{~nm}$ as calculated by Griem (Eqn. 2.9), Measures (Eqn. 2.86), Dreiden (Eqn. 2.28), and this work (Eqn. 2.85) $(\delta \lambda=0.0031 \mathrm{~nm}$ for dye laser). The fringe shift interpretation curves for a selection of other $|\Delta \lambda|$ 's is given in Appendix 3. Notice that the value of Eqn. 2.85 is the same as Measures' value for lower fringe shifts, while it increases to $77 \%$ larger than Measures' value at 8 fringe shifts. For $|\Delta \lambda|=0.010 \mathrm{~nm}$ though, the difference in the four interpretation values at 8 fringe shifts is less than $3 \%$, making any of the equations valid for $0.3 \mathrm{eV}$. 


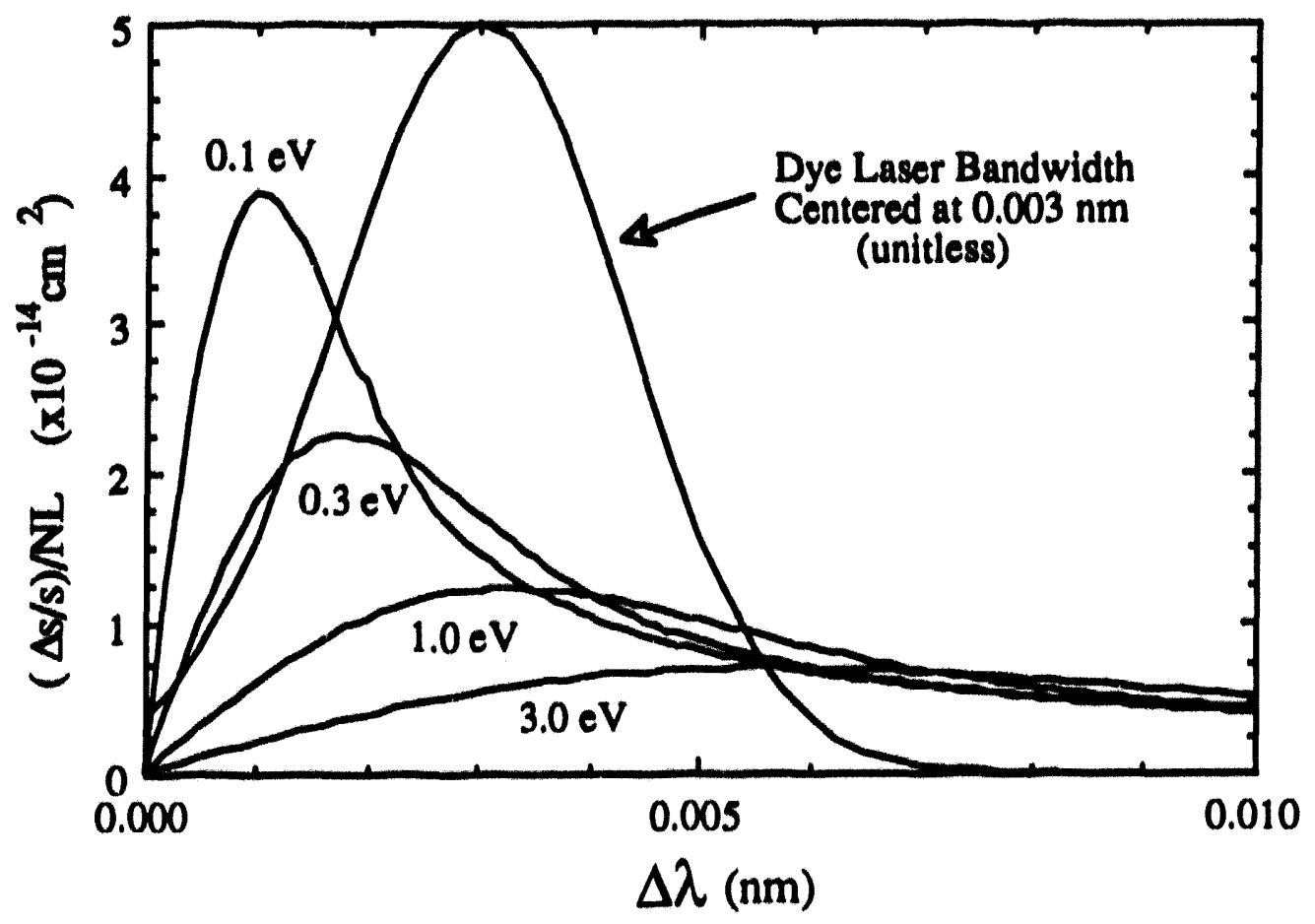

Fig. 2.5 Comparison of the Dye Laser Bandwidth and the Resonant Hologram Interpretation Curves

For the dye laser used in this work, the intensity of the laser beam has a Gaussian-shaped wavelength spread with a FWHM of $0.0031 \mathrm{~nm}$, however there are theoretically at least a few photons of all wavelengths. The higher the line-density, the more absorption of photons with wavelengths near the center of the resonance, and thus the average $|\Delta \lambda|$ that passes through the plume increases with line-density. In turn, a larger $|\Delta \lambda|$ means a smaller fringe shift as observed on the hologram.

Countering this effect is the Doppler shift caused by the energetic plume, as seen by the dye laser beam. Doppler shift causes the laser bandwidth to effectively broaden in the reference frame of the ablated particles. This more heavily weights the small number of photons with 


\section{Table 2.1 Example Parameter Values}

\section{Input:}

$$
\begin{aligned}
& \text { Al(I), } 394.401 \mathrm{~nm} \\
& f=0.115 \\
& \gamma=1.47 \times 10^{8} \mathrm{~Hz} \\
& \Delta \lambda=0.003 \mathrm{~nm} \\
& T=0.3 \mathrm{eV} \\
& \sigma=1.60 \times 10^{10} \mathrm{rad} / \mathrm{sec} \\
& (\delta \lambda=0.0031 \mathrm{~nm}) \\
& \mathrm{NL}=4 \times 10^{14} \mathrm{~cm}^{-2}
\end{aligned}
$$

\section{Qutput:}

$$
\begin{aligned}
& \Delta=1.554 \\
& \alpha=0.00314 \\
& \beta=2.34 \times 10^{10} \mathrm{rad} / \mathrm{sec} \\
& A=2.314 \times 10^{-13} \mathrm{~cm}^{-2} \\
& \varepsilon=0.711-0.658 * \mathrm{I} \\
& \text { F.S. }=4.43
\end{aligned}
$$

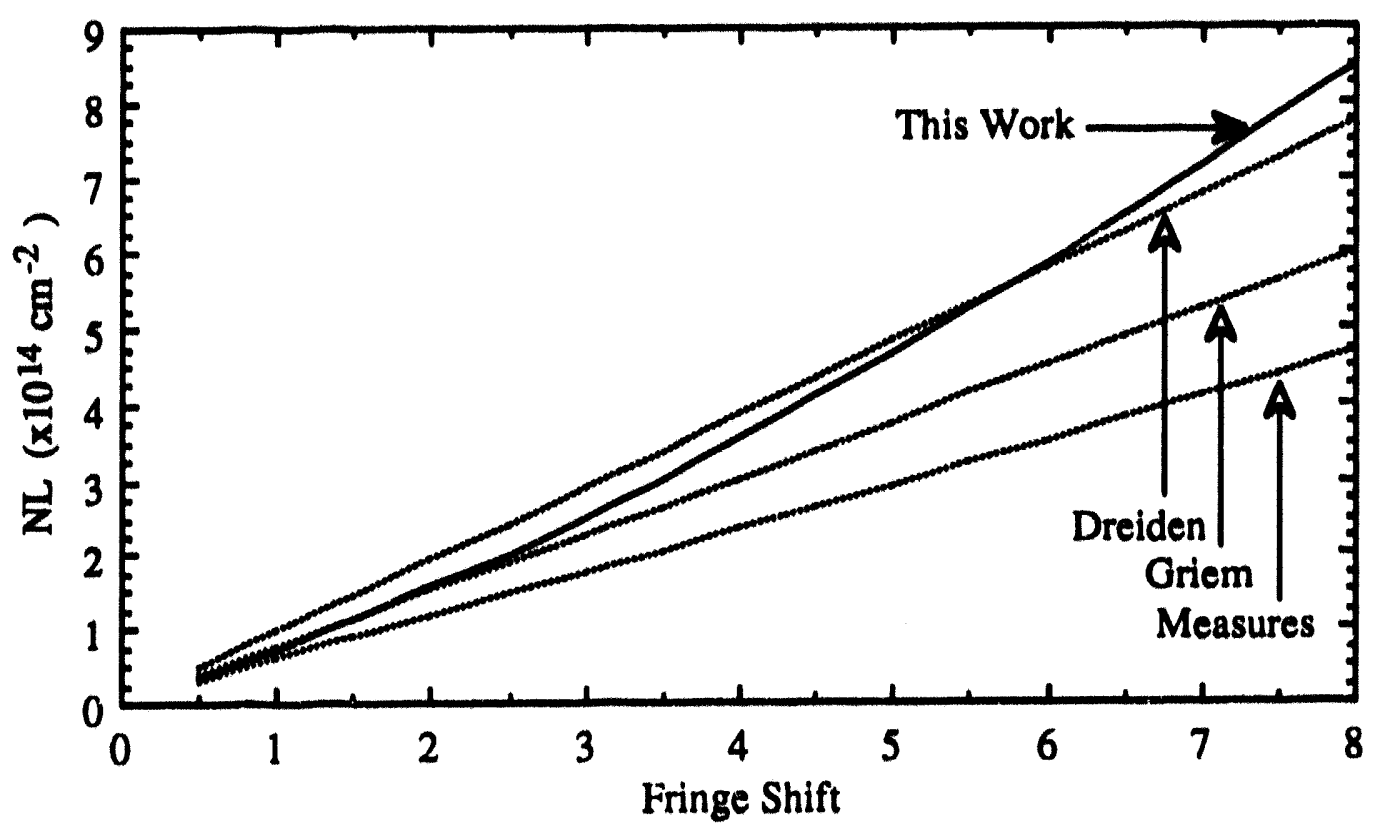

Fig. 2.6 Fringe shift interpretation at $0.003 \mathrm{~nm}$ for the $\mathrm{Al}(\mathrm{I})$, $394.401 \mathrm{~nm}$ line assuming a plume temperature of $0.3 \mathrm{eV}$. 
wavelengths near the peak of the curve in Fig. 2.5, and thus the observed fringe shift increases (when ignoring absorption).

For example, notice in Fig. $2.6(|\Delta \lambda|=0.003 \mathrm{~nm})$ that for a line density of $4 \times 10^{14} \mathrm{~cm}^{-2}$, Griem predicts an observed fringe shift (FS) of $\approx 5.3$. Measures predicts a higher value, $\approx 7.0 \mathrm{FS}$, when accounting for the laser bandwidth broadening caused by Doppler shift while assuming an infinitesimal laser bandwidth to begin with and ignoring absorption. On the other hand, Dreiden predicts a lower value, $\approx 4.3 \mathrm{FS}$, when accounting for the experimental laser bandwidth but ignoring absorption and Doppler shift; i.e., he assumed the plume was cold. Fig. 2.5 implies that a cold plume $(\mathrm{T}<0.1 \mathrm{eV})$ would give less fringe shift at $|\Delta \lambda|=0.003 \mathrm{~nm}$ than a $0.3 \mathrm{eV}$ plume because the peak sensitivity of the diagnostic would be at $|\Delta \lambda|<0.001 \mathrm{~nm}$. Finally, this work predicts a value of $\approx 4.4 \mathrm{FS}$, which takes into account the experimental laser bandwidth, the Doppler shift effects of a hot plume, and the selective absorption of the laser beam as a function of wavelength. In light of this, Fig. 2.6 shows that when considering the ablation plume to be hot, Doppler shift effects dominate at lower line densities and selective absorption effects dominate at higher line-densities.

To understand the temperature dependance of the hologram interpretation calculations, Measures' interpretation is graphed in Fig. 2.7(a) for four temperatures and $|\Delta \lambda|=0.003 \mathrm{~nm}$, and this work's interpretation is similarly graphed in Fig. 2.7(b). (Similar graphs for other $|\Delta \lambda|$ 's are shown in Appendix 3.) The temperature dependence is more pronounced in Measures' calculation because the laser bandwidth is infinitesimal, while the temperature dependence is averaged out between $0.1 \mathrm{eV}$ and $1.0 \mathrm{eV}$ due to the laser bandwidth in this work's calculation. 


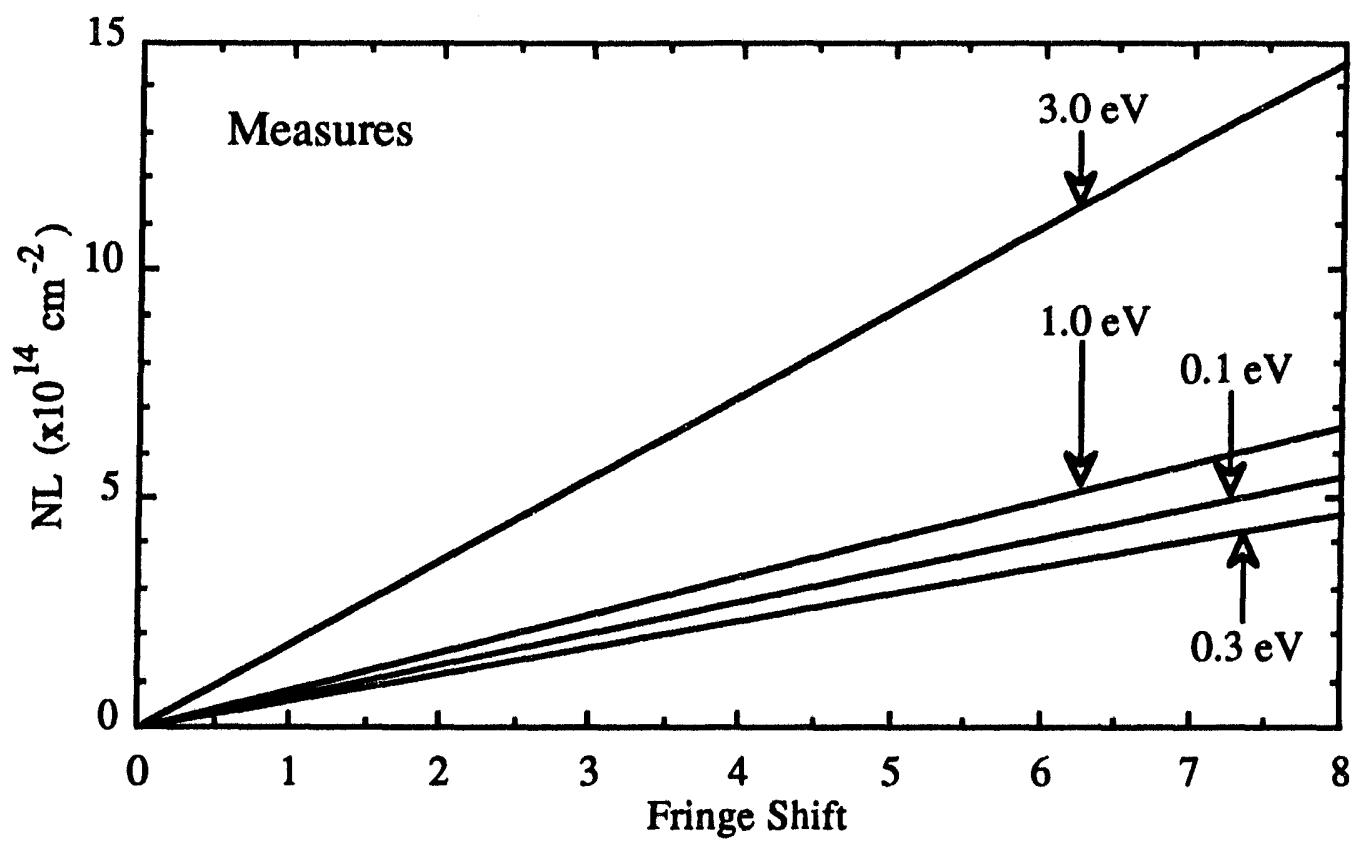

Fig. 2.7(a) Measures' calculation at $0.003 \mathrm{~nm}$ for several ablation plume temperatures.

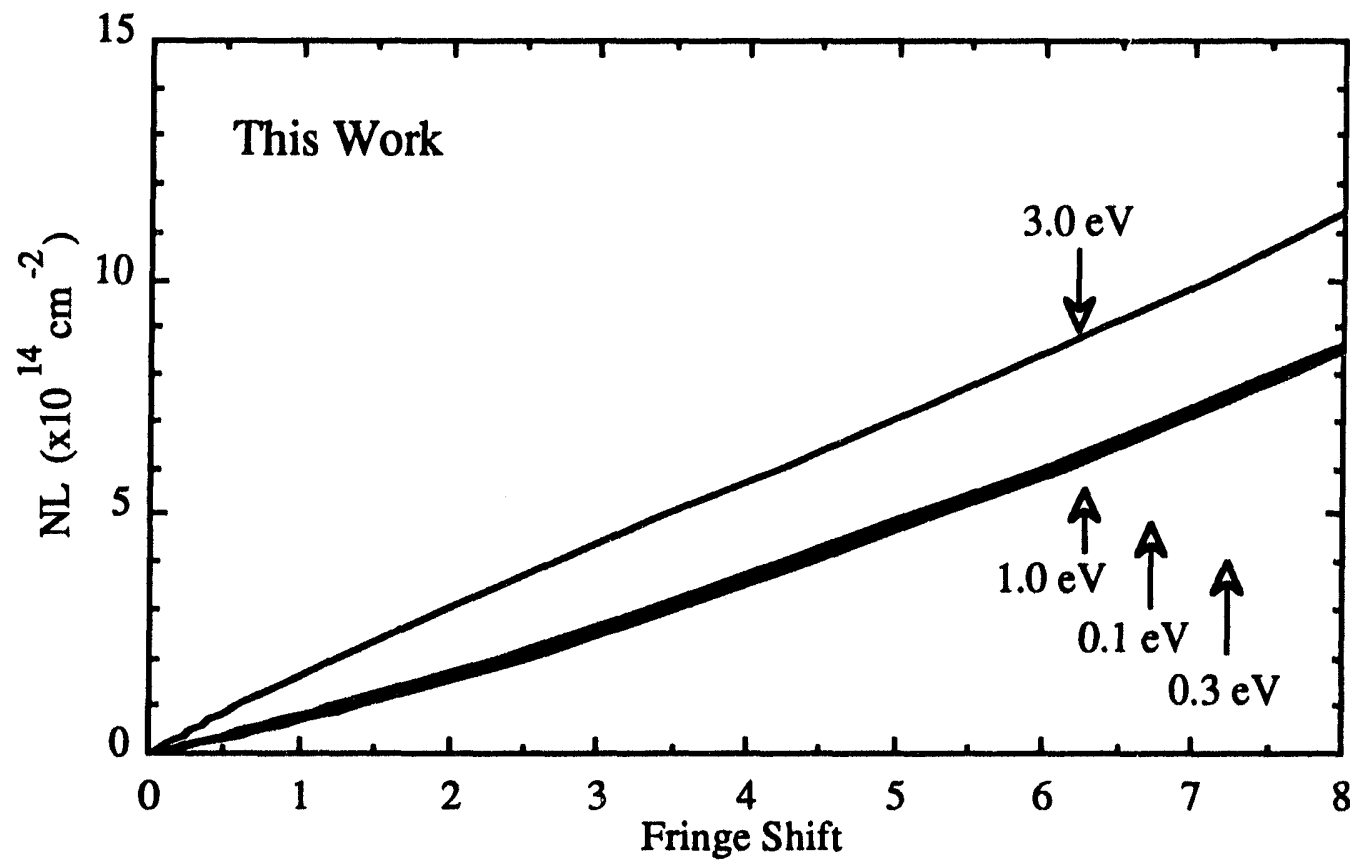

Fig.2.7(b) This work's calculation at $0.003 \mathrm{~nm}$ for several ablation plume temperatures. 
Therefore, the fringe shift interpretation for the plumes in this work is not very sensitive to temperature sirice they are assumed to have parallel expansion velocities in the temperature range $0.1 \mathrm{eV}-1.0 \mathrm{eV}$ (Dre86, Dre91). 


\section{CHAPTER 3 \\ EXPERIMENTAL CONFIGURATION}

\section{The Main Optical Table}

Figure 3.1(a) shows the basic experimental configuration for the optics table utilized for Resonant Holographic Interferometry (RHI) and Dye Laser Resonant Absorption Photography (DLRAP). A Lumonics Excimer Laser (model TE-292-K, $<0.8 \mathrm{~J}, 40 \mathrm{~ns}, 0.5 \mathrm{~Hz}$ max.) filled with a premixed $\mathrm{KrF}$ laser mixture (248 $\mathrm{nm}$ output wavelength) is focused onto a solid aluminum target inside a glass, six-way, vacuum chamber, thus producing an ablation plume. To probe the ablation plume, a Lambda Physik XeCl excimer-pumped dye laser (excimer model LPX105 , dye laser model FL-3002E, $20 \mathrm{~ns}, \approx 5 \mathrm{~mJ}, 50 \mathrm{~Hz}$ max.) is tuned near the $394.401 \mathrm{~nm} \mathrm{Al}(\mathrm{I})$ resonant transition, expanded and collimated, and passed through the ablation plume.

To make an interferogram of the ablation plume, the dye laser beam is first split into two beams by an uncoated, quartz interferometry flat turned $70^{\circ}$ from normal incidence. Due to internal reflections and the dimensions of the quartz flat, there are actually four beams leaving the flat. The primary transmitted beam and the primary reflected beam are utilized for the ablation photography in this work and the ratio of their energies is approximately $4: 1$. The primary transmitted beam is enlarged 28X with a Galilean telescope (FL=17 $\mathrm{mm} \& \mathrm{FL}=480 \mathrm{~mm}$ ) and 


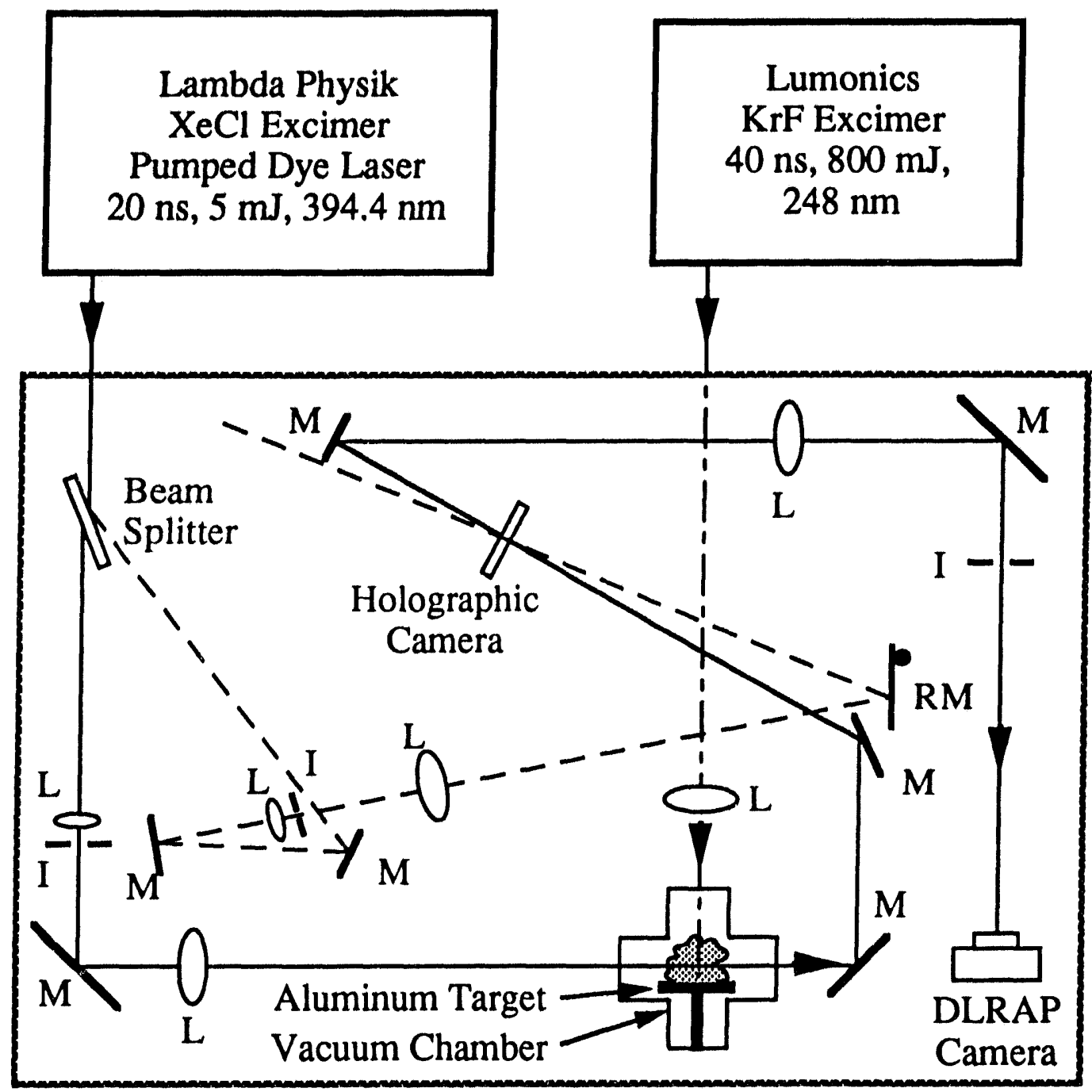

Fig. 3.1(a) Experimental configuration of the main optics table. $\mathrm{I}=$ Iris, $\mathrm{L}=$ Convex Lens, $\mathrm{M}=$ Mirror, $\mathrm{RM}=$ Rotatable Mirror, and BS=Beam Splitter. 


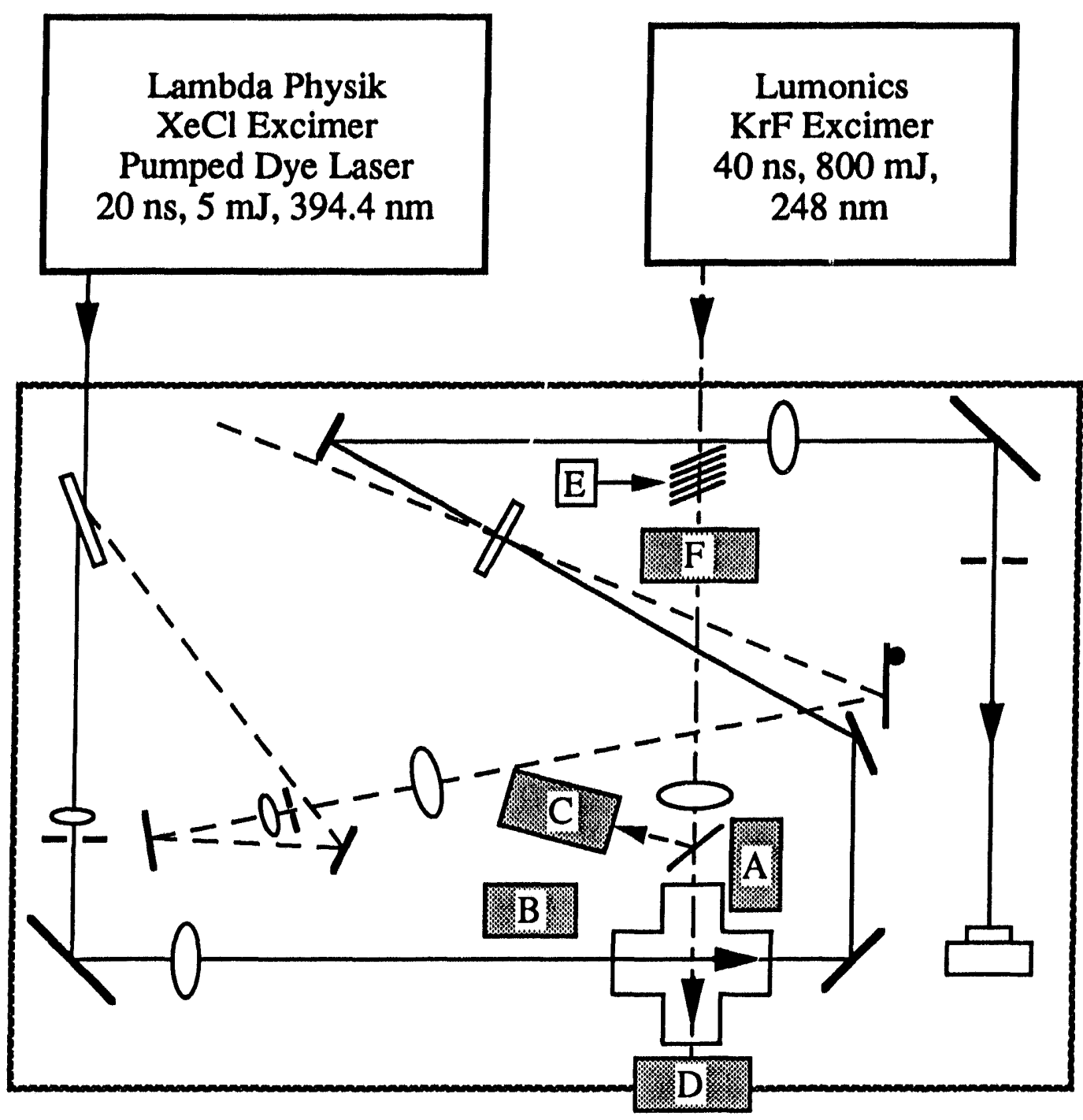

Fig. 3.1(b) Experimental configuration of the main optics table, continued. A silicon photo-diode at (A) measures the timing of the $\mathrm{KrF}$ laser and a p-i-n diode at (B) measures the timing of the dye laser. A thin, quartz flat splits part of the $\mathrm{KrF}$ off onto a calorimeter $(8.8 \mathrm{~V} / \mathrm{J})$ at $(\mathrm{C})$ in order to monitor the shot-to-shot energy of the Lumonics laser. The calorimeter is calibrated after an experiment using a second calorimeter $(0.39 \mathrm{~V} / \mathrm{J})$ at $(\mathrm{D})$ and varying the impinging $\mathrm{KrF}$ laser energy by placing thin quartz flats in the beam at (E). The $\mathrm{KrF}$ laser energy is also monitored during an experimental run by placing the second calorimeter at $(F)$ both before and after the exposure of each holographic plate. 
used as the object beam in the interferometer, passing through the ablation plume in the vacuum chamber. The primary reflected beam is enlarged 16X with a Galilean telescope (FL=17 $\mathrm{mm} \& \mathrm{FL}=280 \mathrm{~mm}$ ) and used as the reference beam. Both beams have an iris located at the focal point in the telescope to remove uncollimated laser light from the photographing process. Mirrors are oriented to direct the collimated beams onto the holographic plate (Agfa-Gavert 8E56, sensitive to $350 \mathrm{~nm}$ - $560 \mathrm{~nm}$ light) and to create approximately equal traveling distances between the beam splitter and the holographic plate. In order for an interference pattern to form at the holographic plate, it is necessary that the difference in these pathlengths be less than coherence length of the laser beam, which is estimated to be about $5 \mathrm{~cm}$. A $5.1 \times 5.1 \mathrm{~cm}$ blue glass filter with peak transmission at $400 \mathrm{~nm}$ is mounted on the $4.4 \times 4.4 \mathrm{~cm}$ entrance window of the holographic camera to keep out excess room light during the experiment.

The experimental procedure is as follows. First, the dye laser is pulsed without the Lumonics excimer laser firing (thus no ablation plume) to expose the holographic plate with a without-the-plume interference pattern. Next, the rotatable mirror in the reference beam is turned very slightly to move the reference image $\approx 1 \mathrm{~mm}$ on the holographic plate. Then the $\mathrm{KrF}$ and dye lasers are pulsed to form a second interference pattern on the holographic plate, this pattern containing the ablation plume information. Four such double-pulsed holographic interferograms are taken on eàch $4 \times 5$ inch holographic plate before the holographic camera is reloaded.

Careful attention is given to the temperature of the dye laser grating during an experimental run. The wavelength output calibration 
(the dye laser control unit readout versus the actual output wavelength) changes $0.0006 \mathrm{~nm} /{ }^{\circ} \mathrm{C}$, which is $20 \%$ of the $0.0031 \mathrm{~nm}$ FWHM laser intensity bandwidth, so the grating temperature and the room temperature were monitored to the nearest $0.1{ }^{\circ} \mathrm{C}$. For the resonant holograms presented here, the dye laser output was recalibrated (see Chapter 4) if the grating temperature changed by $\approx 1{ }^{\circ} \mathrm{C}$ from the time of the initial output calibration.

For Dye-Laser-Resonance-Absorption-Photography (DLRAP), the holographic camera light-tight-box is removed from the camera holder and the object beam then passes through the blue glass plate, neutral density filters (not shown), and into the DLRAP camera, which uses Polaroid Type 47 film.

Figure 3.1(b) shows the location of the p-i-n photo-diodes and the calorimeters on the optics table. The UV enhanced silicon photo-diode for the Lumonics, (A), and the p-i-n photo-diode for the dye laser, (B), are permanently fixed to the optical table. Their rise-times to the laser pulses are on the same order as the length of the laser pulses, thus allowing the time difference between the peaks of the laser pulses to be measured on an oscilloscope. (For a description of the timing circuitry and an example of the timing signals, see Appendix 4.)

During the experiment, the Lumonics laser energy decreases with time and use, dropping nearly $50 \%$ after 30 - 45 minutes of intermediate use. Usually, $33 \%$ of the Lumonics $\mathrm{KrF}$ gas fill was changed after the exposure of each holographic plate; i.e., about every 10 - 20 minutes. Therefore, the Lumonics energy was usually allowed to drop $\approx 25 \%$ during the course of exposing the four, double-pulse holograms for each holographic plate. To monitor the shot-to-shot Lumonics laser energy, a 
portion of the beam is split off with a thin quartz flat onto a sensitive calorimeter $(8.8 \mathrm{~V} / \mathrm{J})$, located at position $(\mathrm{C})$. The peak of the calorimeter signal is then proportional to the total energy impinging onto the aluminum target.

To calibrate calorimeter (C) after each experiment, the target and the back flange of the vacuum chamber were removed from the Lumonics beam path and a second, large-area calorimeter $(0.39 \mathrm{~V} / \mathrm{J})$ was placed behind the vacuum chamber at position (D). The signals on both calorimeters were recorded as a function of the number of thin, quartz flats placed in the beam path at position $(E)$, and thus as a function of laser energy. (For a description of the calorimetry circuitry, see Appendix 4. For an example of the calorimetry signals and of a calibration curve, see Appendix 5.) To provide a day-to-day monitoring of the Lumonics energy changes, the second calorimeter $(0.39 \mathrm{~V} / \mathrm{J})$ was placed at position (F) both before and after the exposure of each holographic plate.

\section{The Lumonics Laser Spot Shape}

Figure 3.2 shows a photograph of a selection of laser ablated, solid aluminum targets ( $99.8 \%$ pure). Spot \#2 was the result of $>300$ laser pulses in October 1992 and was used for about half of the holographic data presented in this work. The laser intensity profile at that time was fairly uniform. Visually, the ablation spot size appears to grow with the number of incident ablation pulses. While the center of the beam etches the target noticeably on the first pulse, the etching due to the wings of the ablating laser takes more pulses to visually notice. (e.g., spot \#1 


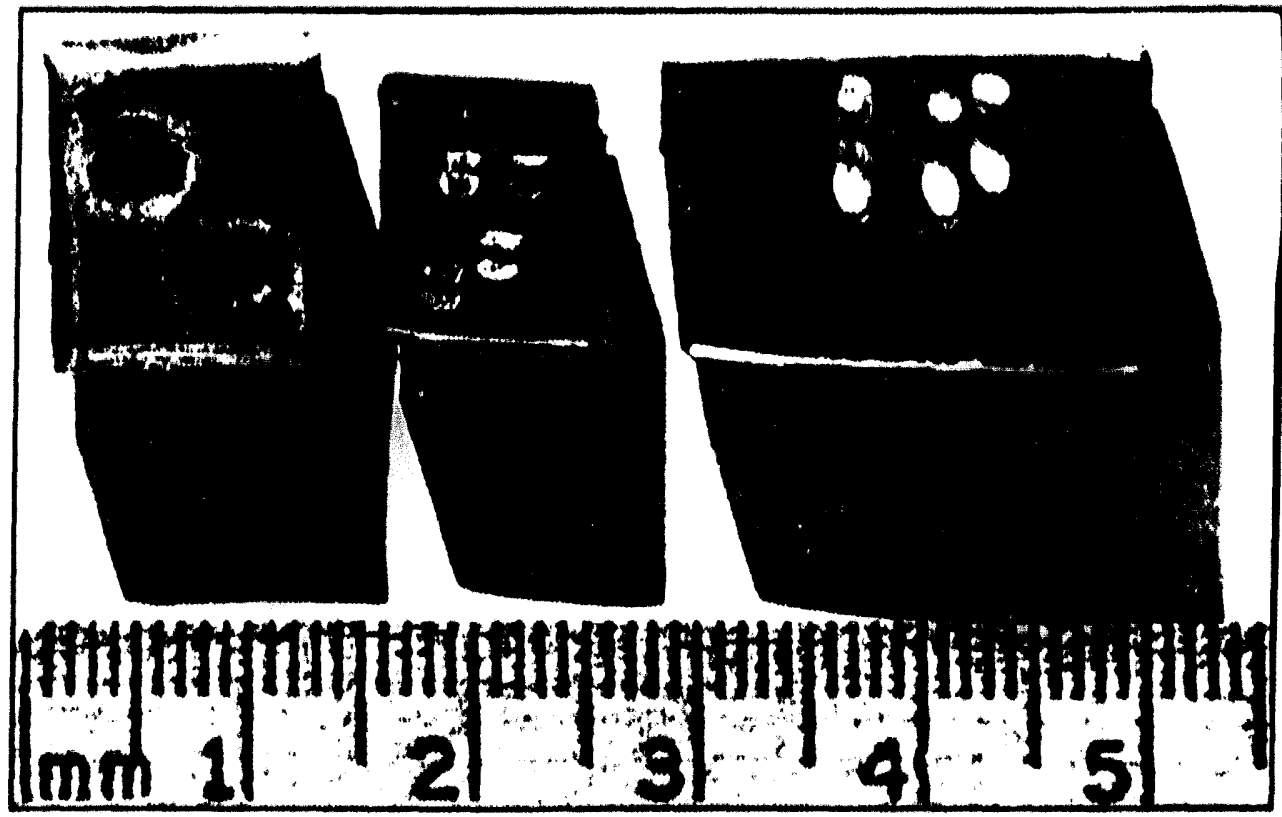

(a)

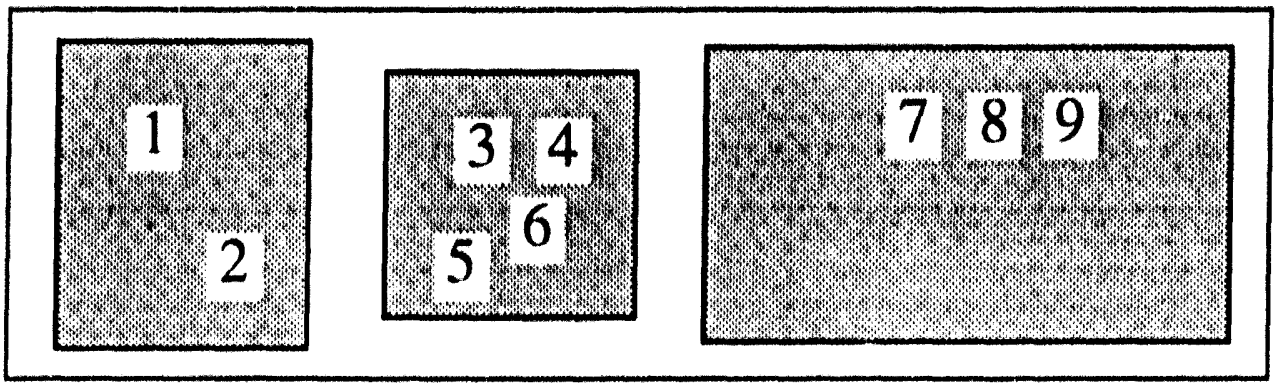

(b)

Fig. 3.2 (a) is a photograph of three, $99.8 \%$ pure aluminum targets used in KrF laser ablation experiments and (b) labels each of the ablation spots. Spots \#1 - \#2 were taken in October 1992 and are representative of laser spots from the period July through December, 1992. Spot \#2 was etched with more than 300 ablation pulses and a spot size of $0.10 \mathrm{~cm}^{2}$ was measured for it, slightly smaller than the visible area showing because the apparent spot size increases with the number of ablation pulses. Spots \#3 - \#6 were taken in March 1993 and are representative of laser spots from the period January through June, 1993. Note the double hot spot in the KrF laser intensity profile. Spot \#3 was etched with about 50 ablation pulses and a spot size of $0.065 \mathrm{~cm}^{2}$ was measured for it. Spots $\$ 7$. \#9 were taken in June 1993 with the aluminum target inclined 69 " 10 the $\mathrm{KrF}$ laser beam. Spot \#9 was etched with 55 ablation pulses and a spot size of $0.049 \mathrm{~cm}^{2}$ was measured for it. 
appears smaller than spot \#2). Therefore a spot size slightly smaller than the actual spot (\#2) was used for the fluence calculations for the October 1992 data $\left(0.10 \mathrm{~cm}^{2}\right)$.

In about January 1993, the laser intensity profile of the Lumonics laser changed and remained so through the rest of the data taken for this work. It exhibited two distinct hot spots of unequal intensity, as in spots \#3-\#6. Attempts to prevent one of the two spots from hitting the target while still obtaining a detectable fringe shift using RHI were unsuccessful, so the laser spot was used as is. Although noni of the spots on the center target correspond to particular holograms shown in this work, it is the most clear example of ablated target spots for the data taken between January and June, 1993. Much of the holographic data taken in this time period is not presented in this work because there were often two, unequal plumes expanding away from the target; thus the holograms presented from that time period are the least affected by the double-spot laser intensity profile.

Again the appearance of the ablation spot changes with the number of pulses. Spot \#5 is due to only several pulses and shows only the beginning of material removal from the surface. Spots \#3 and \#6 are due to more shots, and they show the double craters that form with that particular laser intensity profile. Spot \#4, which was made with the most ablation pulses of the four spots, shows a ring of soot around the spot due to redeposition of ablated material. For the purpose of fluence calibrations, the size ablation spot was measured to be the size of a rectangle just encompassing the two laser spots (e.g., $0.065 \mathrm{~cm}^{2}$ for spot $\# 3$ for $\approx 50$ ablation pulses). 
The Lumonics twin-spot was exaggerated for the deposition experiment because the target was tilted 690 to allow the Lumonics laser beam access to the target. The silicon wafer was placed $1.5 \mathrm{~cm}$ away from the ablation target and 55 ablation pulses formed spot \#9, which was used for both the deposition holograms presented here as well as the forming of the aluminum thin film on the wafer. The wafer is $\langle 100\rangle$ crystalline silicon coated with $115 \mathrm{~nm}$ of $\mathrm{SiO}_{2}$ followed by $493 \mathrm{~nm}$ of amorphous silicon. The aluminum thin film was deposited on the amorphous silicon layer. Here, the spot size for the fluence calibration was determined to be the sum of the two separate ablation areas for spot $\# 9\left(0.049 \mathrm{~cm}^{2}\right)$.

\section{Developing And Reconstructing The Holograms}

Once the holographic plates were exposed, they were developed in a darkroom using the procedure given in Table 3.1. They were then reconstructed with an expanded HeNe laser using the setup shown in

Table 3.1

Photographic developing process for the holographic plates.

1) $3.6 \mathrm{~min}$.

2) $30 \mathrm{sec}$.

3) $4 \mathrm{~min}$.

4) $10 \mathrm{sec}$.

5) $4 \mathrm{~min}$.

6) $30 \mathrm{sec}$.

7) $30 \mathrm{sec}$.
D-19 Photographic Developer

(visually verify proper development using darkroom lighting)

Stop Bath

Rapid Fixer

Water (continue using regular

Hypoclear lighting)

Water

Photoflo 


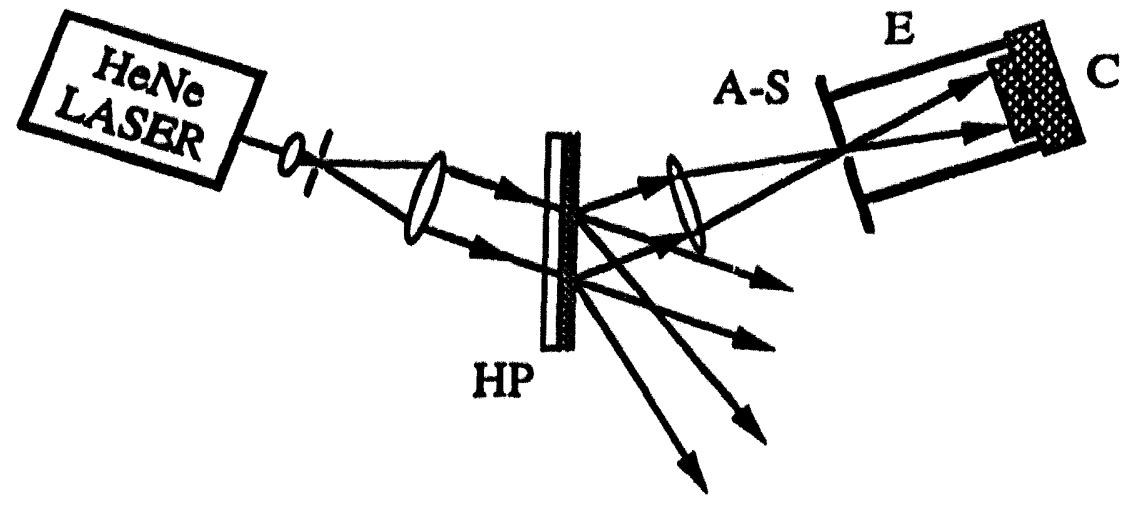

Figure 3.3 Schematic For Reconstructing The Hologram (see Fig. 2.2). HP=Holographic Plate, $\mathrm{C}=$ Camera, $\mathrm{E}=$ Light Tight Enclosure, and A-S=Aperture and Shutter.

Fig. 3.3. The holographic plate orientation with respect to the laser is reversed from the direction of exposure to utilize a more intense reconstructed image of the plume. Reconstruction with a laser wavelength other than the exposure wavelength does not change the image (for a transmission hologram), just the image location and magnification (Ves79). The reconstructed image was focused through a camera shutter (which doubled as an iris) and into a camera using Polaroid 667 film was placed in the path of the reconstructed image. The shutter speed was adjusted to vary the exposure (and thus the contrast) of the image on the film.

\section{Plume Expansion In RF/Plasma Environments}

Figure 3.4 shows the experimental setup to produce a steady-state (for $<10 \mathrm{sec}$ ) RF-plasma in the region of plume expansion in order to 
observe the effects of an RF-plasma on plume expansion. Since this is the focal point of Chi Hong Ching's forthcoming dissertation, Fig. 3.4 is shown here as an example of an application of RHI and its explanation will be brief.

A $7 \mathrm{MHz}, \approx 50 \mathrm{~W}$, RF generator was connected to a pair of $8.4 \mathrm{~cm}$ diameter, brass, parallel plates separated by $4.8 \mathrm{~cm}$ inside the the vacuum chamber. The aluminum target face was positioned so that it was just slightly outside the parallel plates. This allowed the plume to expand between the parallel plates while not letting the target significantly perturb the electric field between the plates.

The turbopump port valve was closed $99 \%$ and argon gas was leaked onto the vacuum chamber through a leak valve (not shown) to obtain a constant pressure of 1 Torr. The RF voltage was turned on for about 3 seconds before the ablating laser was fired, thus allowing the RF circuitry and the plasma sufficient time to equilibrate. 


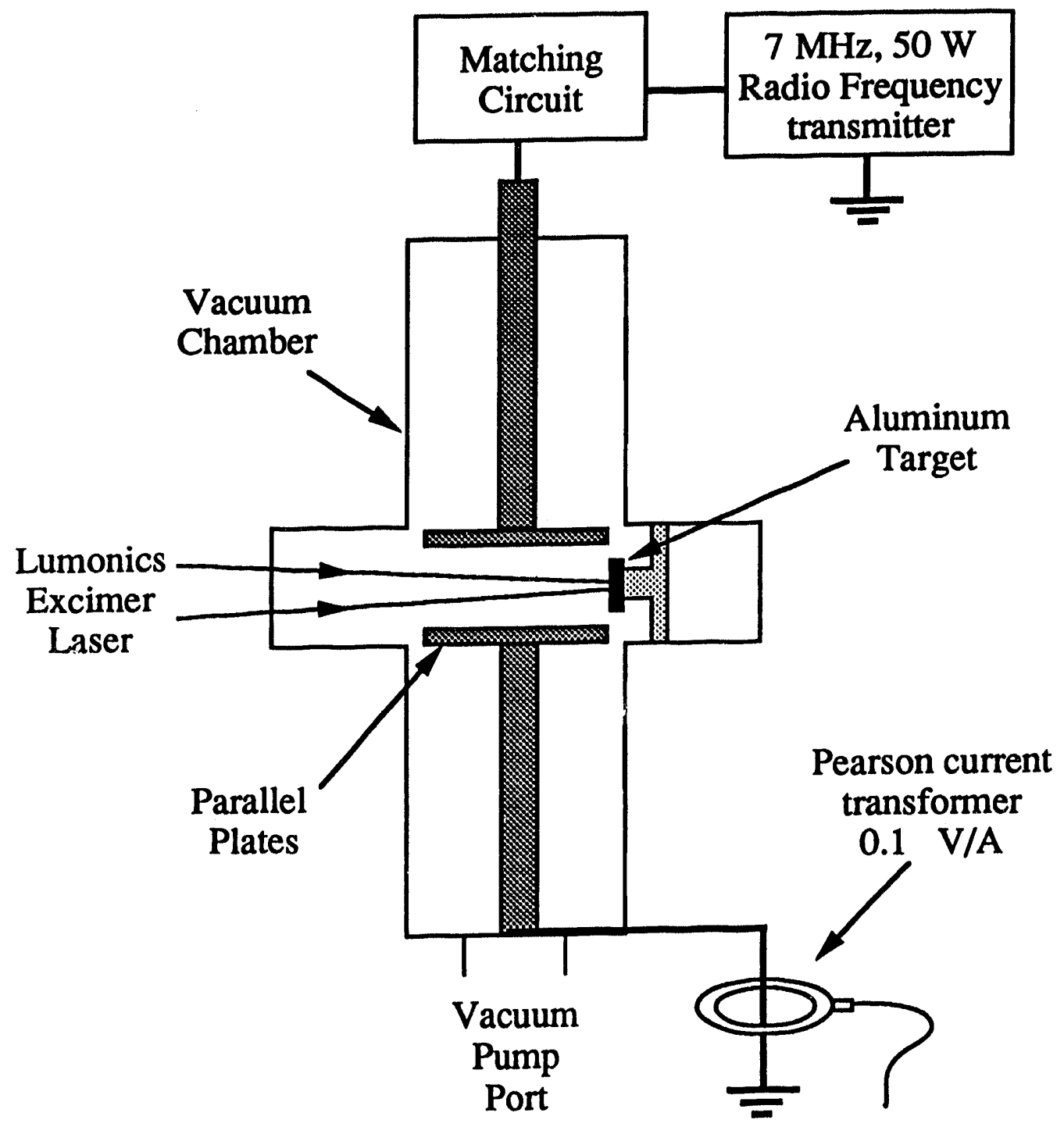

Fig. 3.4 Schematic of the RF-Argon-Plasma setup. The brass, parallel plates have a diameter of $8.4 \mathrm{~cm}$ and are separated by $4.8 \mathrm{~cm}$. The plasma current is measured using the Pearson transformer. 


\section{CHAPTER 4 \\ EXPERIMENTAL RESULTS AND DISCUSSION}

\section{Determining $\lambda_{0}$}

To determine the center of the atomic resonance with respect to the dye laser wavelength control unit, a piece of paper is placed in the object beam path while the wavelength is scanned near the resonance center during $\mathrm{KrF}$ laser ablation of an aluminum target. The absorption image of the plume on the paper is observed to determine the wavelength of most absorption and then four holograms (one holographic plate) are taken across the anticipated line center using the smallest wavelength increment available on the grating control unit $(0.001 \mathrm{~nm}-0.002 \mathrm{~nm})$. The reference beam mirror is turned in the same direction for these four holograms for ease of interpretation.

Figure 4.1 shows four sample holograms in a 1.0 Torr argon background, at $2.5 \mu \mathrm{s}$, and with an ablation laser fluence of $\approx 2.3 \mathrm{~J} / \mathrm{cm}^{2}$. First of all, holograms 4.1(b) and 4.1(c) show fringe shift toward the target while hologram 4.1(d) shows fringe shift away from the target. This confirms the change in the index of refraction from $n<1$ to $n>1$ when crossing from one side to the other side of the line center (see Fig 2.3). (The polarity of the change will be experimentally determined in the next section.) Secondly, hologram 4.1(c) has the greatest amount of absorption. Therefore, hologram $4.1(\mathrm{c})$ is chosen as $\Delta \lambda=0.000 \mathrm{~nm}$ for 


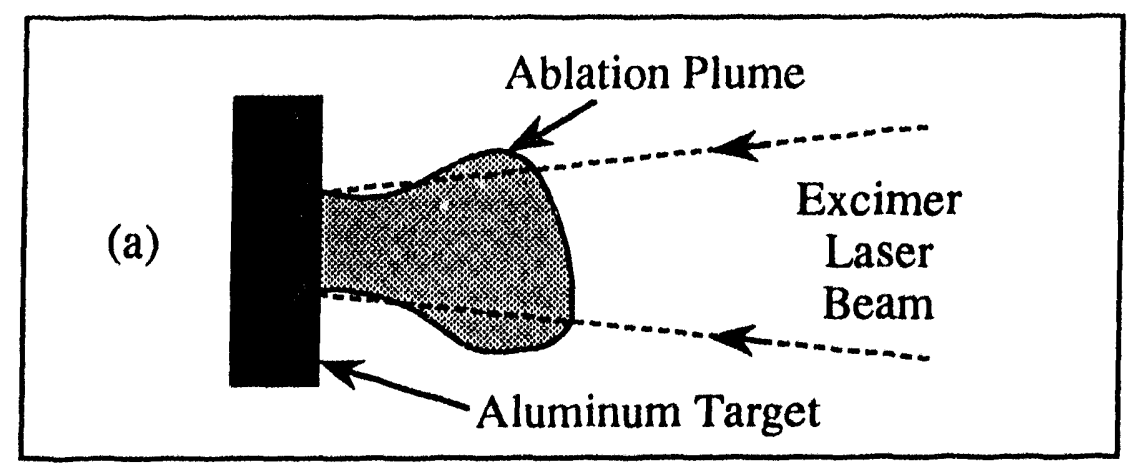

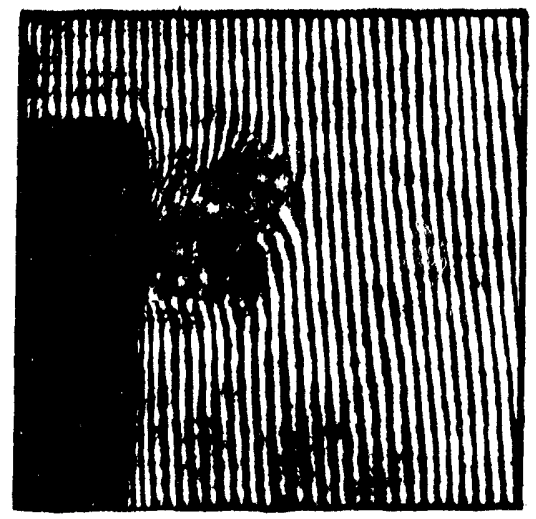

(b)

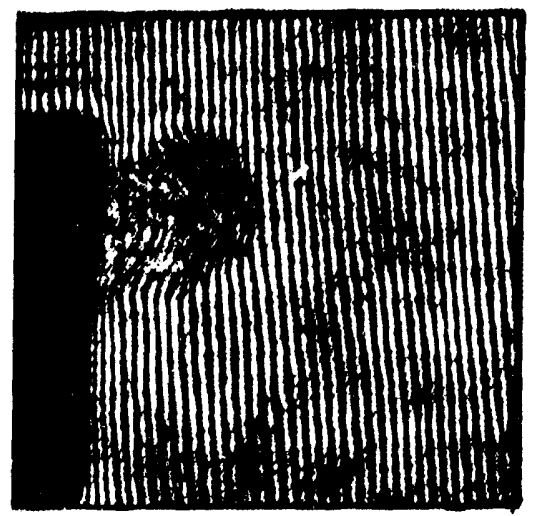

(d)

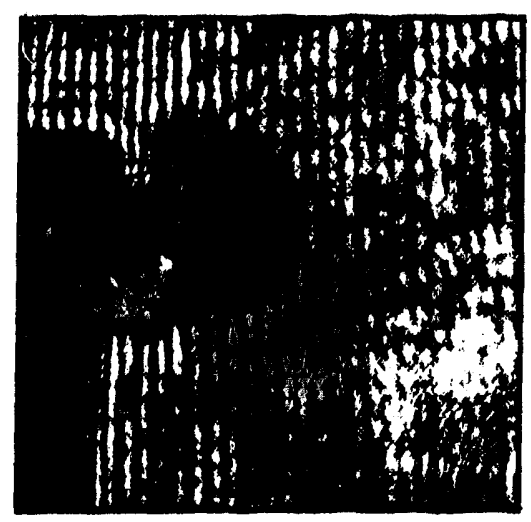

(c)

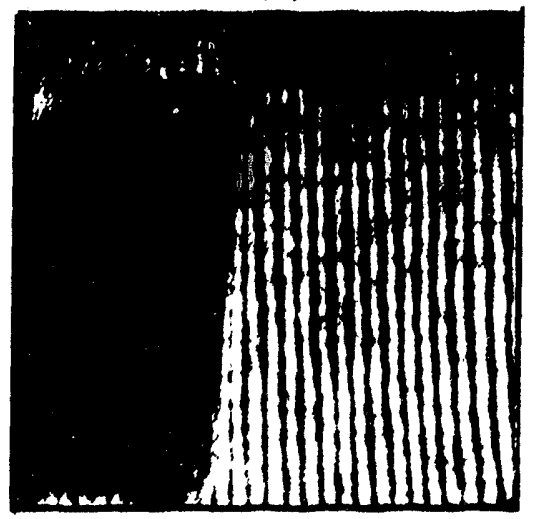

(e)

Fig. 4.1 Calibrating the dye laser to the aluminum line at $394.401 \mathrm{~nm}$. (a) Photographic orientation; holograms (b), (c), (d) and (e) were taken in 1 Torr Argon gas at $\left(2.50 \mu \mathrm{s}, \approx 2.3 \mathrm{~J} / \mathrm{cm}^{2}\right)$. (b), (c) and (d) were taken successively with a wavelength increment of $0.001 \mathrm{~nm}$. Since (c) has the most absorption and the fringe shift direction changes between (c) and (d), hologram (c) was chosen to be line center, $\Delta \lambda=+0.000 \mathrm{~nm}$. Holograms (b) and (d) thus have calibrated wavelengths of $-0.001 \mathrm{~nm}$ and $+0.001 \mathrm{~nm}$, respectively. Hologram (e) shows no fringe shift at $\Delta \lambda=+0.493 \mathrm{~nm}$ and thus all fringe shift in (b), (c) and (d) is due to resonant effects. (From laser spot \#2 of Fig. 3.2(b).) 
the purposes of interpreting holograms within this data run. Hologram 4.1(e) has a large $\Delta \lambda,+0.493 \mathrm{~nm}$, and no fringe shift, thus showing that the fringe shift seen in holograms 4.1(b)-4.1(d) are due only to resonant effects.

For better fringe shift interpretation, it was chosen to turn the reference beam mirror in such a direction as to have the fringes shift away from the target. Therefore, for $\Delta \lambda<0$ the mirror was turned in one direction while for $\Delta \lambda>0$ the mirror was turned in the other direction.

\section{Resonant Refraction Effects}

Figure 4.2 shows the resonant refraction effects on holograms for operating on both sides of the line center. These effects are important because, while some light is passing through the ablation plume unrefracted, the light passing through the more dense regions of the plume may be refracted. The refracted and nonrefracted light can then interact and alter the fringe appearance of the holograms.

Figure 4.2(a) shows that for $n>1$, the laser ablation plume acts similar to a convex glass lens and focuses the light toward the center of the plume. Thus, if the most dense region of the ablation plume has separated from the target surface, the edges of the plume will appear unaltered while the center of the plume will appear to be a confusing pattern of black and white, as in Fig. 4.2(c).

Figure 4.2(b) shows that for $n<1$, the laser ablation plume acts similar to a concave glass lens (or a convex plasma) and defocuses the light away the center of the plume. Thus, if the most dense region of the ablation plume has separated from the target surface, the center of the 

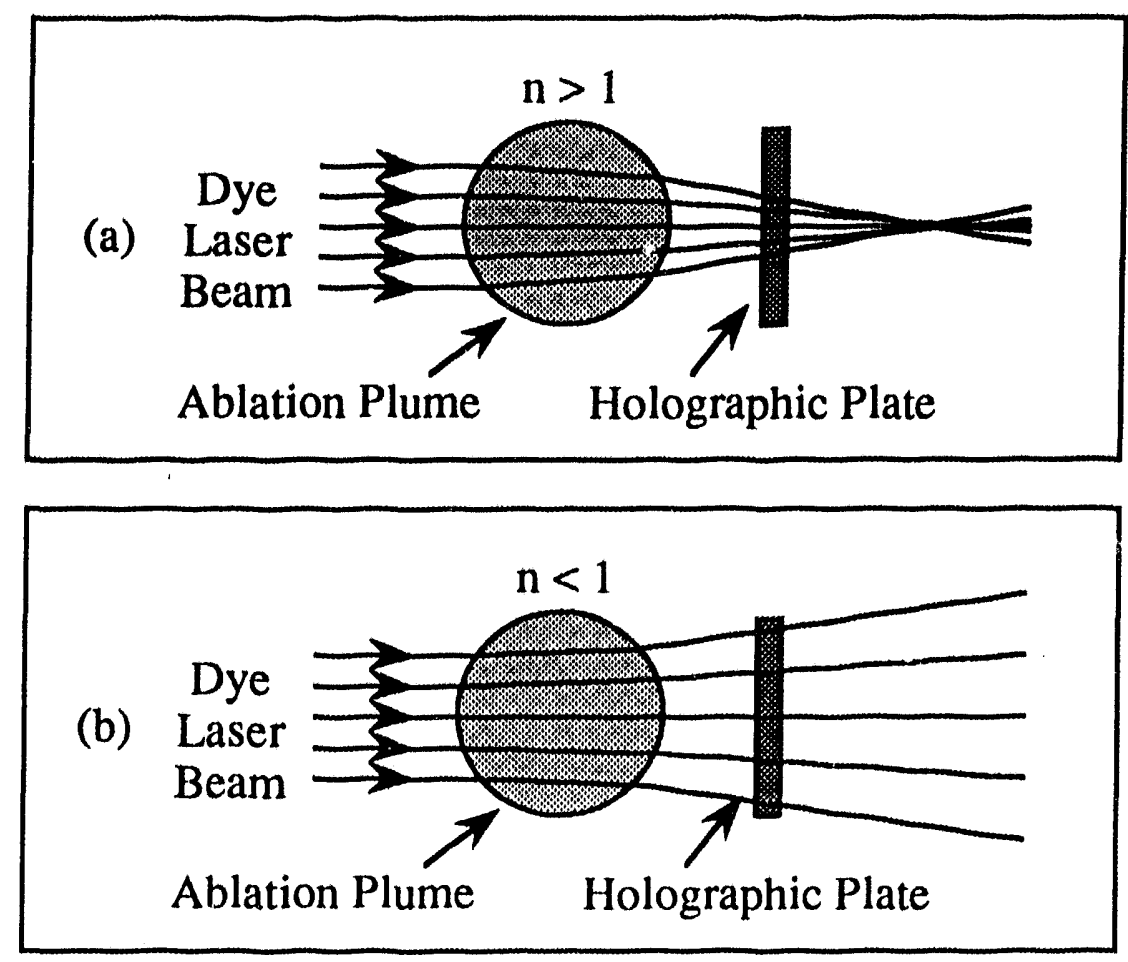

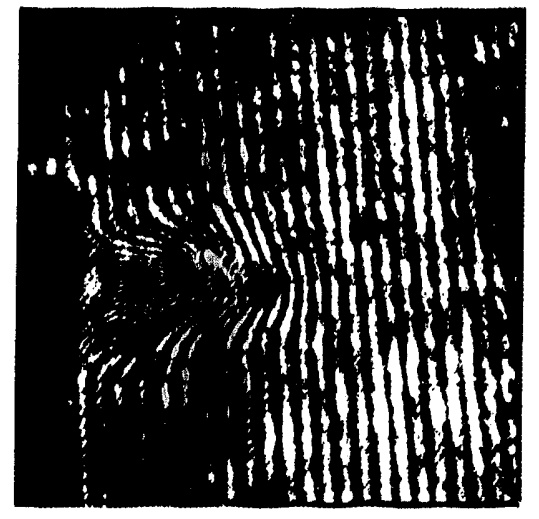

(c)

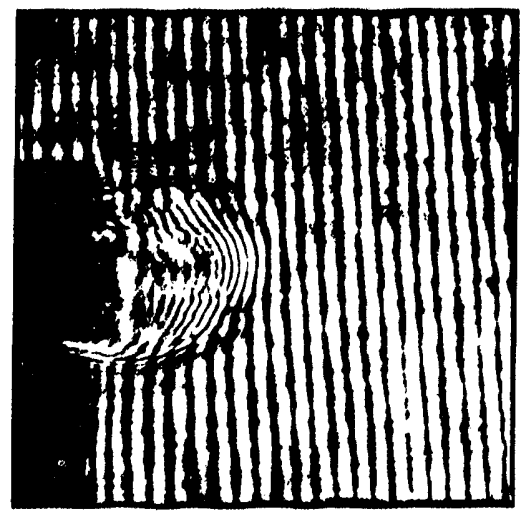

(d)<smiles>CCCCC(C)(C)C</smiles>

Fig. 4.2 (a) and (b) show refraction effects of the dye laser beam encountering an ablation plume with $(n>1)$ and $(n<1)$, respectively (Ves79). Refraction effects are enhanced near resonance as evidenced by holograms (c) and (d), taken in vacuum at $0.50 \mu \mathrm{s}$ and $\approx 2.4 \mathrm{~J} / \mathrm{cm}^{2}$. Hologram (c) was taken with $+0.004 \mathrm{~nm}$ and exhibits crisscrossing fringes at its center, thus the ablation plume had $(n>1)$. Hologram (d) was taken with $-0.003 \mathrm{~nm}$ and exhibits non-fringe lines encircling the plume and light located on the target in the hologram, thus the ablation plume had $(n<1)$. (From laser spot \#2 of Fig. 3.2(b).) 
plume will appear unaltered (or dimly lit) while the edges of the plume will appear to be altered, as in Fig. 4.2(d). Note the light that appears on the target in the hologram at the left and right sides of the plume which can only come from refraction away from the plume center. Given the calibrated wavelengths of Figs. 4.2(c) and (d) are $+0.004 \mathrm{~nm}$ and -0.003 $\mathrm{nm}$, respectively, the polarity of the phase shift response curve is then experimentally confirmed to be

$$
\operatorname{sgn}\{\operatorname{Re}[\tilde{\mathbf{n}}-1]\}=-\operatorname{sgn}\left\{\omega-\omega_{0}\right\}
$$

in agreement with Eqn. 2.25 and as shown in Fig. 2.3.

For the purposes of better fringe shift interpretation, a large enough $|\Delta \lambda|$ was chosen to attempt to minimize resonant refraction effects while retaining the resonant particle sensitivity of smaller $|\Delta \lambda|$ 's. Also, it is possible that the resonant refraction effects could be decreased by properly imaging the ablation plume during the reconstruction process, assuming that the refracting region in the ablation plume was small (i.e., on the order of the depth of focus for the imaging setup).

\section{Fringe Shift Interpretation}

Figure 4.3 shows the basic method for interpreting holographic interferometry fringe shifts. The holograms are enlarged $200 \%$ using a photocopier and the fringes are traced by hand onto a clean sheet of paper placed on a light board. Non-fringe features of the hologram, such as refraction, diffraction, absorption and uninterpretable or distracting features, are thereby removed from the working image. In the region of the ablation plume, the unshifted fringes are also drawn onto the fringe tracing, as shown by the dashed lines in Fig. 4.3(a). 


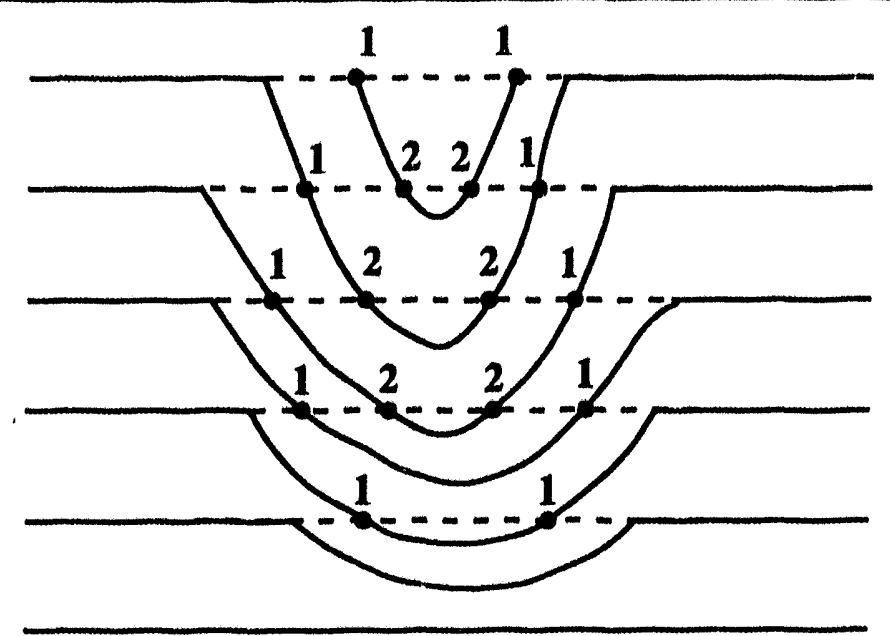

(a)

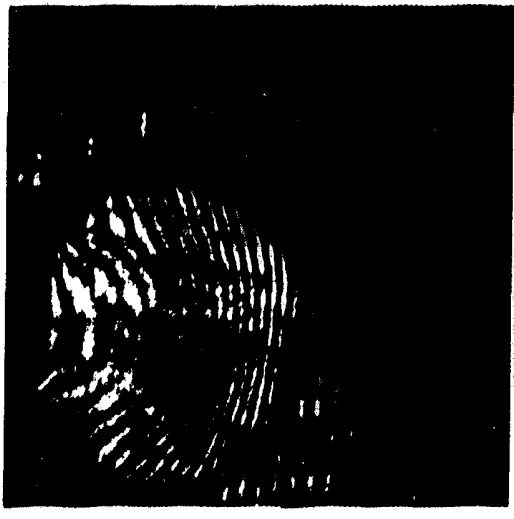

(b)

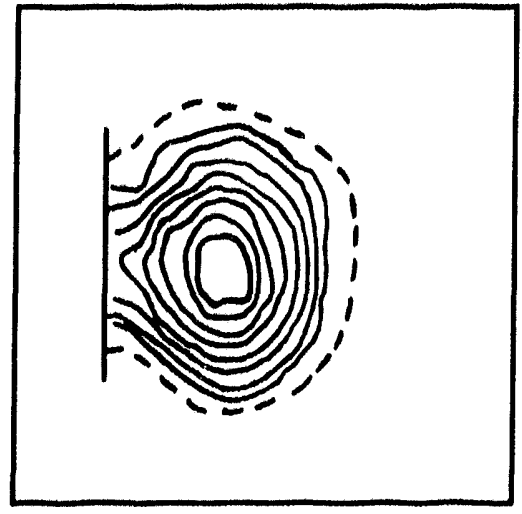

(c)

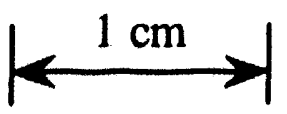

Fig. 4.3 (a) shows the method of fringe shift interpretation (Ves79). The points of incremental fringe shift are determined along the unshifted, dotted lines by their intersection with other shifted fringes and then, not shown here, the equi-fringe shift points are connected with a smooth line to form equi-fringe shift contours. Hologram (b) was taken in 1 Torr Argon gas at $\left(4.00 \mu \mathrm{s},-0.006 \mathrm{~nm}, \approx 2.4 \mathrm{~J} / \mathrm{cm}^{2}\right)$. (c) is an equicontour plot of (b) where the dashed line is a shock wave and the solid lines correspond to increasing fringe shifts; from outer to inner line: (0.5 FS $\left.=7.0 \times 10^{13} \mathrm{~cm}^{-2}\right),\left(1.0 \mathrm{FS}=1.4 \times 10^{14} \mathrm{~cm}^{-2}\right),\left(1.5 \mathrm{FS}=2.1 \times 10^{14} \mathrm{~cm}^{-2}\right),(2.0 \mathrm{FS}$ $\left.=2.9 \times 10^{14} \mathrm{~cm}^{-2}\right),\left(2.5 \mathrm{FS}=3.6 \times 10^{14} \mathrm{~cm}^{-2}\right),\left(3.0 \mathrm{FS}=4.4 \times 10^{14} \mathrm{~cm}^{-2}\right)$, $\left(3.5 \mathrm{FS}=5.2 \times 10^{14} \mathrm{~cm}^{-2}\right)$, and $\left(4.0 \mathrm{FS}=6.0 \times 10^{14} \mathrm{~cm}^{-2}\right.$ ). (From laser spot \#2 of Fig. 3.2(b).) 
Next, the photocopy of the hologram is removed from the light board and a new sheet of paper is placed over the first tracing. The points of equal phase shift are determined and recorded on the new sheet, and then these points are connected with a smooth curve. In Fig. 4.3(a) for example, all of the points labeled " 2 " are recorded on the overlaying sheet of paper and then connected with an oval passing though each of the six points. To determine half-fringe shifts, the points where the fringe shifts halfway between two reference fringes (dashed lines) is used.

Figure 4.3(b) and (c) show an example of a hologram at $4.00 \mu \mathrm{s}$ in 1 Torr argon gas, and its equi-line-density-contour plot. The dashed line represents the shock wave and the solid lines represent incremental 0.5 fringe shifts, to a maximum of 4.0 fringe shifts at the center of the plume, which corresponds to a maximum aluminum neutral line-density of $6.0 \times 10^{14} \mathrm{~cm}^{-2}$ for this hologram. Again, all of the aluminum neutral line-densities presented in this work assume a constant kinetic temperature of $0.3 \mathrm{eV}$ in a Maxwellian ablation plume in quasiequilibrium.

Unfortunately, there are several points where uncertainties are introduced in the interpretation process. First of all, uncertainties are introduced in the process of interpreting the black and white pattern on the hologram to determine what are fringes versus what are refraction and diffraction effects. Areas near the center of ablation plumes sometimes have a certain amount of absorption or resonant refraction effects that brought uncertainty into the location of the shifted fringes, including what would appear to be additional fringe lines in the center of the ablation plume. Either these areas were not interpreted at all or human discernment was used to interpret the recorded information. 
Secondly, uncertainties are introduced in the process of choosing the location of the unshifted reference fringes (dashed lines). The reference fringes are actually slightly curved due to the dye laser beam wavefront not being of constant phase over the entire expanded beam, so an uncertainty of up to 0.25 fringe shift is introduced in this step. Third, uncertainties are introduced in the process of accurately tracing the points of equal fringe shift and, finally, in the process of interpretively drawing a smooth curve between the points. Digital fringe shift recording systems (such as CCD cameras) may be able to reduce uncertainty in the "tracing" process but complex fringe shift interpretation programs are needed to distinguish between fringe shift, refraction, and diffraction. For this reason, the holograms in this work were interpreted by tracing.

\section{Shot-To-Shot Consistency}

Figure 4.4 shows a set of ten holograms taken in vacuum at the same time, $0.51-0.53 \mu$ s, with approximately the same ablating $\mathrm{KrF}$ laser fluence, $2.2-2.6 \mathrm{~J} / \mathrm{cm}^{2}$, and with the same wavelength, $\Delta \lambda=-0.013 \mathrm{~nm}$. This set of tracings shows that there is a wide range of variability in the shape of the ablation plume even when the experimental conditions are supposedly the same. Fig. 4.4 also demonstrates that the size of the ablation plume is somewhat dependent on fluence. The tracings are in the order of fluence and are, in general, larger for higher fluences. The differences between the holograms are most likely due to the changing laser intensity profile from shot-to-shot and to some degree the changes in the ablation surface caused by previous ablation pulses. It is also possible that the plumes had different temperatures, which would 


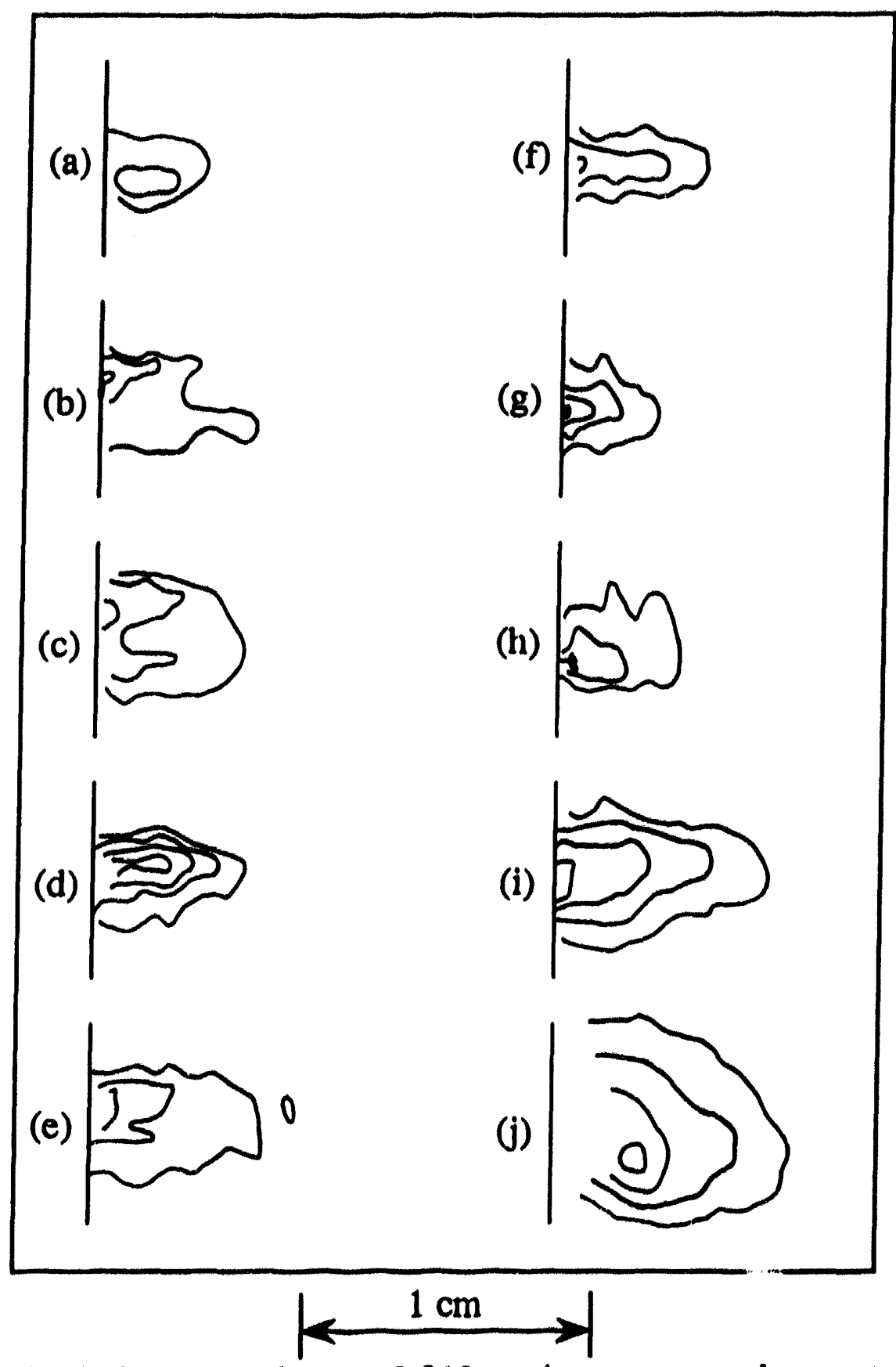

Fig. 4.4 Ten holograms taken at $-0.013 \mathrm{~nm}$ in vacuum to demonstrate shot-to-shot consistency. Lines correspond to increasing fringe shifts; from outer to inner: $\left(0.5 \mathrm{FS}=1.6 \times 10^{14} \mathrm{~cm}^{-2}\right),\left(1.0 \mathrm{FS}=3.2 \times 10^{14} \mathrm{~cm}^{-2}\right)$,

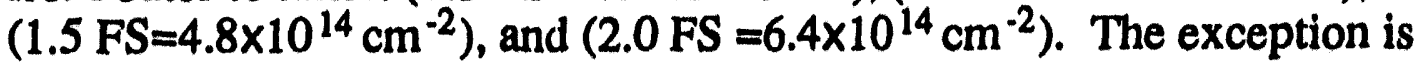
(a) where both solid lines correspond to $0.5 \mathrm{FS}$ and the line-density is nearly zero in the center of the ablation plume. (a) and (b) were at $\approx 2.2$ $\mathrm{J} / \mathrm{cm}^{2}$, (c), (d), (e), (f), (g) and (h) were at $\approx 2.3 \mathrm{~J} / \mathrm{cm}^{2}$, (i) was at $\approx 2.5$ $\mathrm{J} / \mathrm{cm}^{2}$, and (j) was at $\approx 2.6 \mathrm{~J} / \mathrm{cm}^{2}$. Note that $(\mathrm{b})$, (c) and (e) show possible effects of the double focal spot of the $\mathrm{KrF}$ laser. (From similar to laser spots \#3-\#6 of Fig. 3.2(b).) 


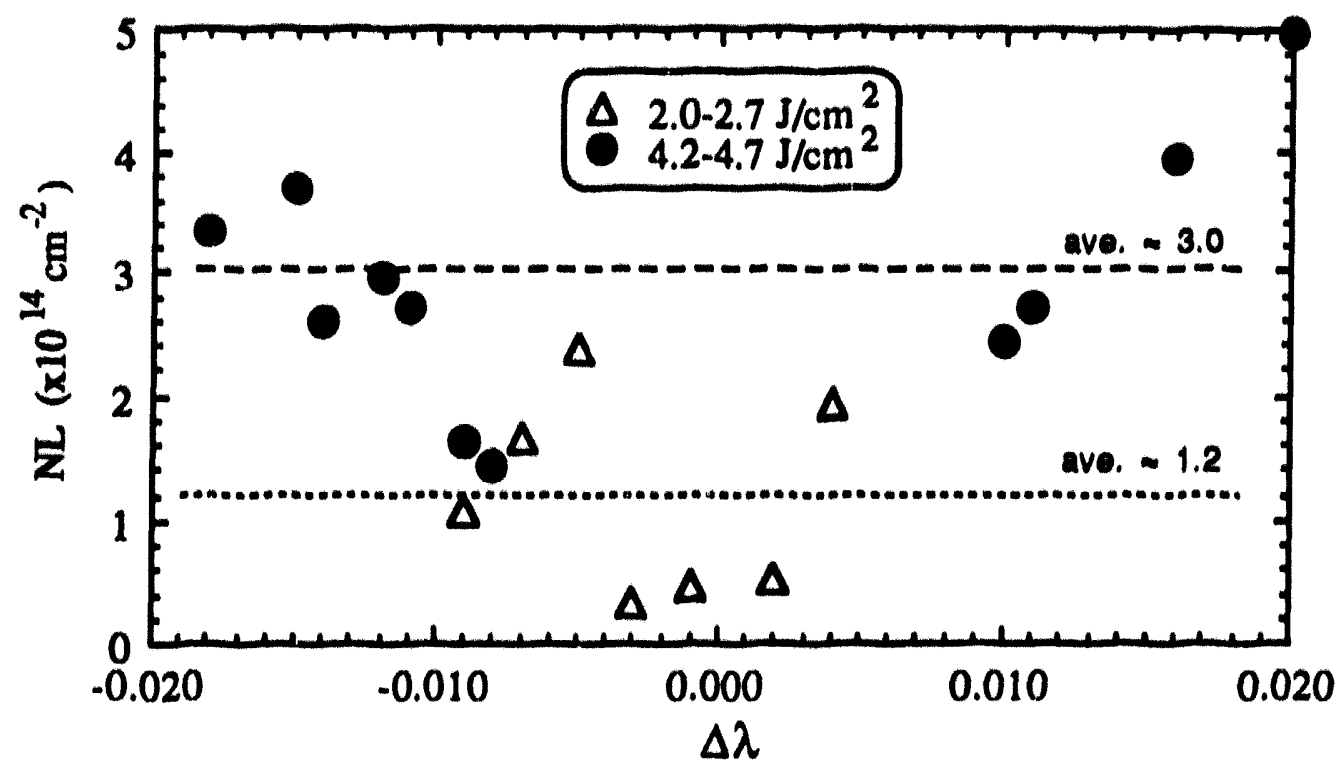

Fig. 4.5 Line-density versus wavelength at $0.5 \mu$ s for two fluences to show consistency of line-density measurement as a function of wavelength. (lower fluence: from spot \#2 of Fig. 3.2(b); higher fluence: from similar to spots \#1 - \#2 of Fig3.2(b))

produce some variation in the observed fringe shift. The variations shown in Fig. 4.4 mean that hologram interpretation of a set of holograms where one parameter is varied can only be approximate at best.

Two series of holograms were taken at $0.5 \mu \mathrm{s}$ at various wavelengths in order to determine the consistency of measuring the linedensity for an ablation plume over a range of dye laser wavelengths. The first set of holograms used a fluence of $2.0-2.7 \mathrm{~J} / \mathrm{cm}^{2}$ and the second set used a fluence of $4.2-4.7 \mathrm{~J} / \mathrm{cm}^{2}$. Figure 4.5 shows the results as a function of wavelength for the two scans at a point $0.7 \mathrm{~cm}$ away from the target along the line of symmetry of the ablation plume.

First of all, note that absorption prevented the measurement of fringe shift at $0.7 \mathrm{~cm}$ on the hologram for $|\Delta \lambda|<0.009 \mathrm{~nm}$ for the higher 
laser fluence case. Secondly, note that the measured aluminum neutral line density seemingly increases with increasing $|\Delta \lambda|$. This is because the measured fringe shifts at this point on each of the holograms were around 1.0 fringe shift and were measured to the nearest 0.25 fringe shift. Thus, Fig. 4.5 looks similar to a theoretical curve showing the aluminum neutral line-density interpretation as a function of wavelength for a constant 1.0 fringe shift. Third, note that the ratio of the line-density averages for the two fluences is $\mathbf{2} .5$, which is comparable to, yet larger than, the ratio of the average laser fluences, $\sim 1.9$. This is consistent in principle with the findings of Dreyfus (Dre91), where the LIF signal (and thus the particle density) for copper ablation was experimentally determined to be proportional to $\exp (-1 / \mathrm{F})$ for fluences identical to this work, where $\mathrm{F}$ is the laser fluence, although Dreyfus measured a gain of $\approx 25 \mathrm{X}$ in the LIF signal for the same fluence increase as presented in Fig. 4.5.

It is suggested that if the fringe shifts at an identical point on a series of holograms were measured with greater certainty (such as $5.00 \pm$ 0.25 fringe shifts instead of $1.00 \pm 0.25$ fringe shifts), then the graph of the measured line density versus wavelength would be more constant as a function of wavelength for each fluence. It is also entirely possible that the temperatures in the plumes at the two different fluence ranges could be different, and thus affecting the outcome of Fig. 4.5. A temperature of $0.3 \mathrm{eV}$ was assumed for both fluence ranges since the individual plume temperatures could not be determined at that time. 


\section{Laser Ablation In Vacuum \\ And Low Pressure, Inert, Background Gas}

Resonant holographic interferometry time scans of laser ablation plume expansion, within the fluence range of $2-6 \mathrm{~J} / \mathrm{cm}^{2}$, were taken in vacuum ( $0.2 \mathrm{~m}$ Tnrr air) and in argon gas backgrounds for the pressures 14 mTorr, 52 mTorr, 210 mTorr, 1 Torr, and 35 Torr. In addition, plume expansion in 1 Torr argon plasma environment was explored. The plume expansions as a function of time were very similar for the background gas pressures up to 210 mTorr, so their plume expansion interpretations will be handled together and compared to the laser ablation plume expansion-in-vacuum theory used by Utterback, et al. (Utt76), Talents (Tal83), and Kools, et al. (Koo92). Then the 1 Torr and 35 Torr data will be presented and, finally, the summaries of the data will be presented.

Figure 4.6 shows a time scan in a series of holograms in vacuum. The ablation plume begins close to the surface and expands away from the target at later times. It is assumed that the aluminum neutral atoms experience collisions and that almost all of the electrons have recombined with the atoms at very early times $(<\approx 0.1 \mu \mathrm{s})$. The peak aluminum neutral line-density on each hologram $\left(>5 \times 10^{14} \mathrm{~cm}^{-2}\right)$ is located at the surface at early times $(0.29 \mu \mathrm{s}$ and $0.50 \mu \mathrm{s})$ and then separates from the surface at later times $(0.70 \mu \mathrm{s}, 0.90 \mu \mathrm{s}$, and $1.11 \mu \mathrm{s})$. This corresponds to inferred particle densities of up to $1 \times 10^{15} \mathrm{~cm}^{-3}$. Also, the peak aluminum neutral line density decreases at later times to $\approx 1 \times 10^{14} \mathrm{~cm}^{-2}$ (at $1.11 \mu \mathrm{s})$. 


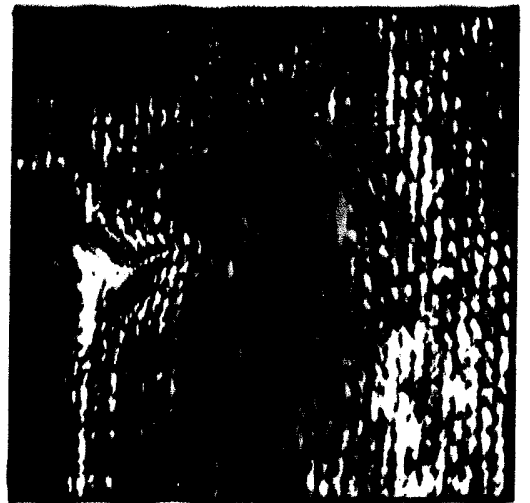

(a)

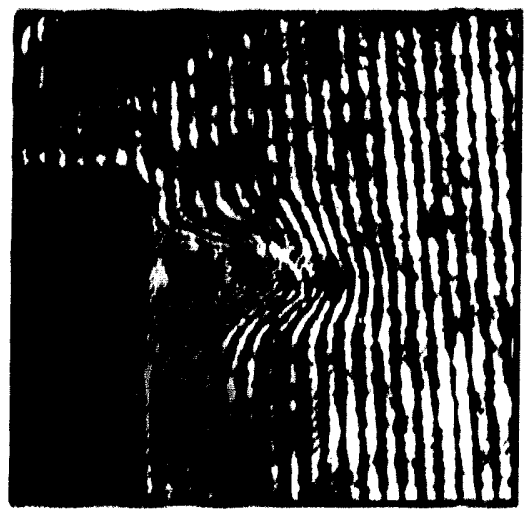

(b)

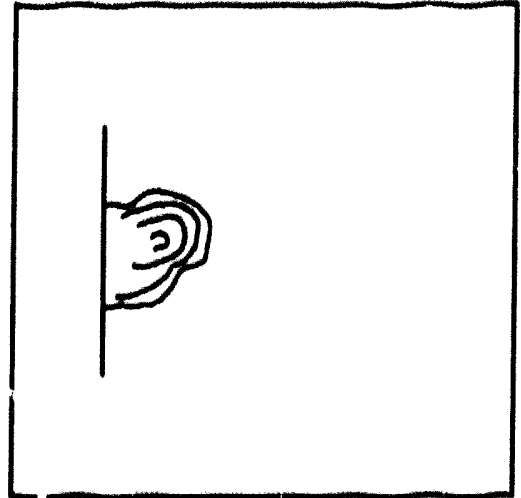

(c)

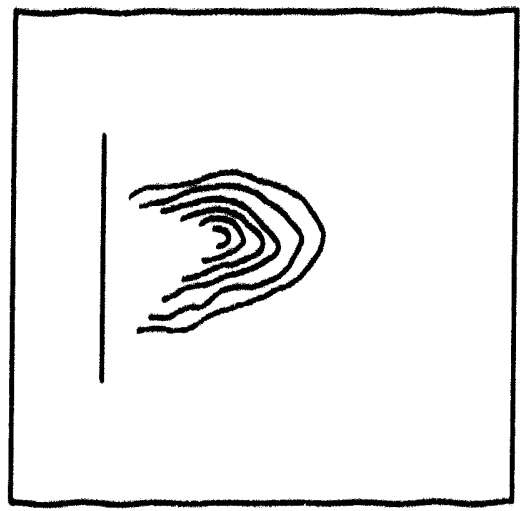

(d)

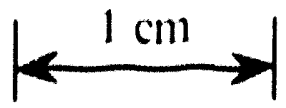

Fig. 4.6 'Time scan in vacuum. Hologram (a) taken at $(0.29 \mu \mathrm{s}$, $\left.+0.004 \mathrm{~nm}, \approx 2.4 \mathrm{~J} / \mathrm{cm}^{2}\right)$ and hologram (b) taken at $(0.50 \mu \mathrm{s},+0.004$ $\mathrm{nm}, \approx 2.4 \mathrm{~J} / \mathrm{cm}^{2}$ ). (c) is an equicontour plot of (a), lines correspond to increasing fringe shifts, from outer to inner line: $\left(0.5 \mathrm{FS}=4.3 \times 10^{13}\right.$ $\left.\mathrm{cm}^{-2}\right),\left(1.0 \mathrm{FS}=9.0 \times 10^{13} \mathrm{~cm}^{-2}\right),\left(2.0 \mathrm{FS}=1.9 \times 10^{14} \mathrm{~cm}^{-2}\right)$, and $(3.0 \mathrm{FS}$ $\left.=3.0 \times 10^{14} \mathrm{~cm}^{-2}\right)$; (d) is an equicontour plot of (b), lines correspond to increasing fringe shifts, from outer to inner line: $\left(0.5 \mathrm{FS}=4.3 \times 10^{13}\right.$ $\left.\mathrm{cm}^{-2}\right),\left(1.0 \mathrm{FS}=9.0 \times 10^{13} \mathrm{~cm}^{-2}\right),\left(2.0 \mathrm{FS}=1.9 \times 10^{14} \mathrm{~cm}^{-2}\right),(3.0 \mathrm{FS}$ $\left.=3.0 \times 10^{14} \mathrm{~cm}^{-2}\right),\left(4.0 \mathrm{FS}=4.2 \times 10^{14} \mathrm{~cm}^{-2}\right)$, and $\left(5.0 \mathrm{FS}=5.5 \times 10^{14} \mathrm{~cm}^{-2}\right)$. (From laser spot \#2 of Fig. 3.2(b).) 


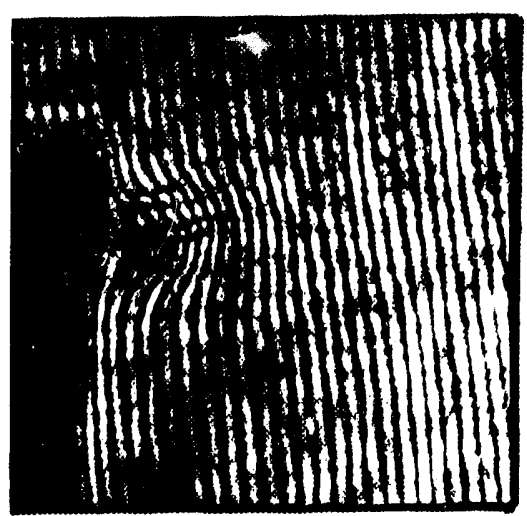

(e)

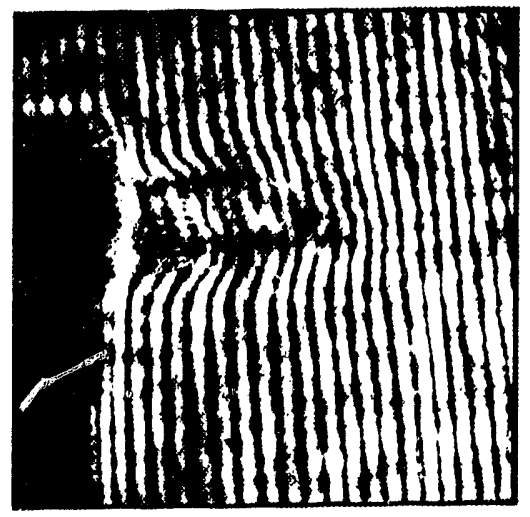

(f)

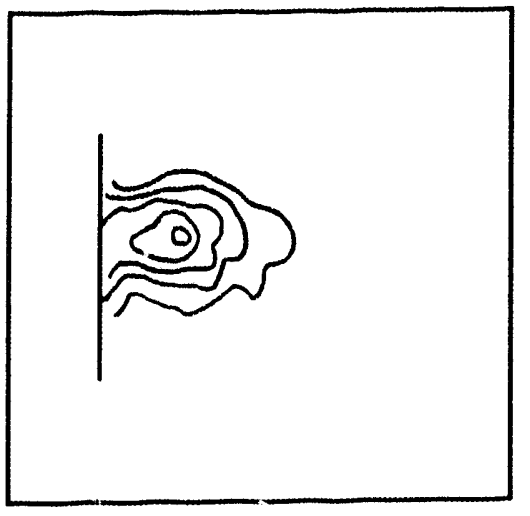

(g)

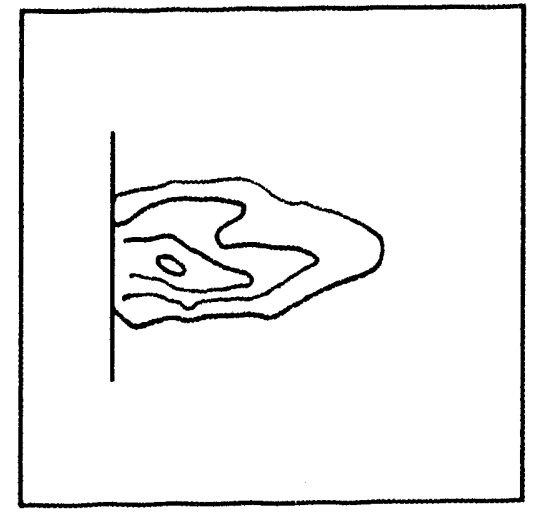

(h)

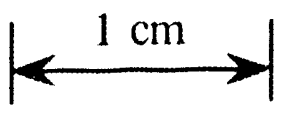

Fig. 4.6 Time scan in vacuum, continued. Hologram (e) taken at $\left(C .70 \mu \mathrm{s},+0.004 \mathrm{~nm}, \approx 2.4 \mathrm{~J} / \mathrm{cm}^{2}\right)$ and hologram (f) taken at $(0.90 \mu \mathrm{s}$, $+\left(1.004 \mathrm{~nm}, \approx 2.4 \mathrm{~J} / \mathrm{cm}^{2}\right)$. $(\mathrm{g})$ is an equicontour plot of $(\mathrm{e})$, lines cc respond to increasing fringe shifts, from outer to inner line: $(0.5 \mathrm{FS}$ $\left.=4.3 \times 10^{13} \mathrm{~cm}^{-2}\right),\left(1.0 \mathrm{FS}=9.0 \times 10^{13} \mathrm{~cm}^{-2}\right),\left(1.5 \mathrm{FS}=1.4 \times 10^{14} \mathrm{~cm}^{-2}\right)$, $\left(2.0 \mathrm{FS}=1.9 \times 10^{14} \mathrm{~cm}^{-2}\right)$, and $\left(2.5 \mathrm{FS}=2.5 \times 10^{14} \mathrm{~cm}^{-2}\right)$; $(\mathrm{h})$ is an equicontour plot of (f), lines correspond to increasing fringe shifts, from outer to inner line: $\left(0.5 \mathrm{FS}=4.3 \times 10^{13} \mathrm{~cm}^{-2}\right),\left(1.0 \mathrm{FS}=9.0 \times 10^{13}\right.$ $\left.\mathrm{cm}^{-2}\right),\left(1.5 \mathrm{FS}=1.4 \times 10^{14} \mathrm{~cm}^{-2}\right)$, and $\left(2.0 \mathrm{FS}=1.9 \times 10^{14} \mathrm{~cm}^{-2}\right.$ ). (From laser spot \#2 of Fig. 3.2(b).) 


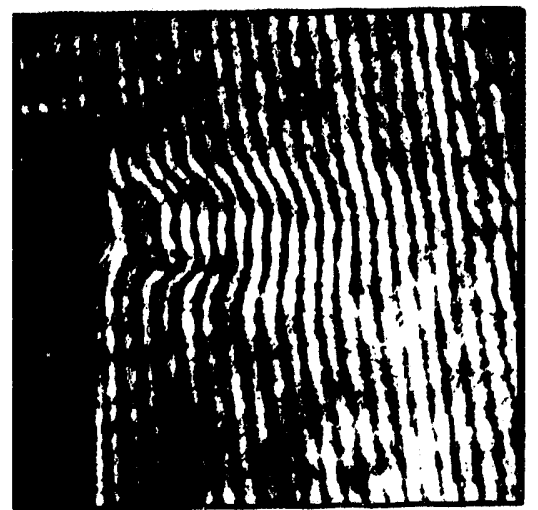

(i)

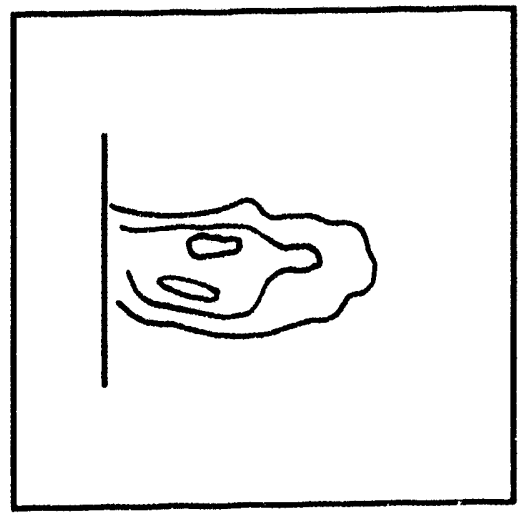

(j)

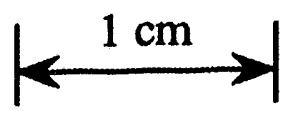

Fig. 4.6 Time scan in vacuum, continued. Hologram (i) taken at $\left(1.11 \mu \mathrm{s},+0.004 \mathrm{~nm}, \approx 2.4 \mathrm{~J} / \mathrm{cm}^{2}\right)$. (j) is an equicontour plot of (i), lines correspond to increasing fringe shifts, from outer to inner line: $\left(0.5 \mathrm{FS}=4.3 \times 10^{13} \mathrm{~cm}^{-2}\right),\left(1.0 \mathrm{FS}=9.0 \times 10^{13} \mathrm{~cm}^{-2}\right)$, and $(1.5 \mathrm{FS}$ $=1.4 \times 10^{14} \mathrm{~cm}^{-2}$ ). (From laser spot \#2 of Fig. 3.2(b).) 


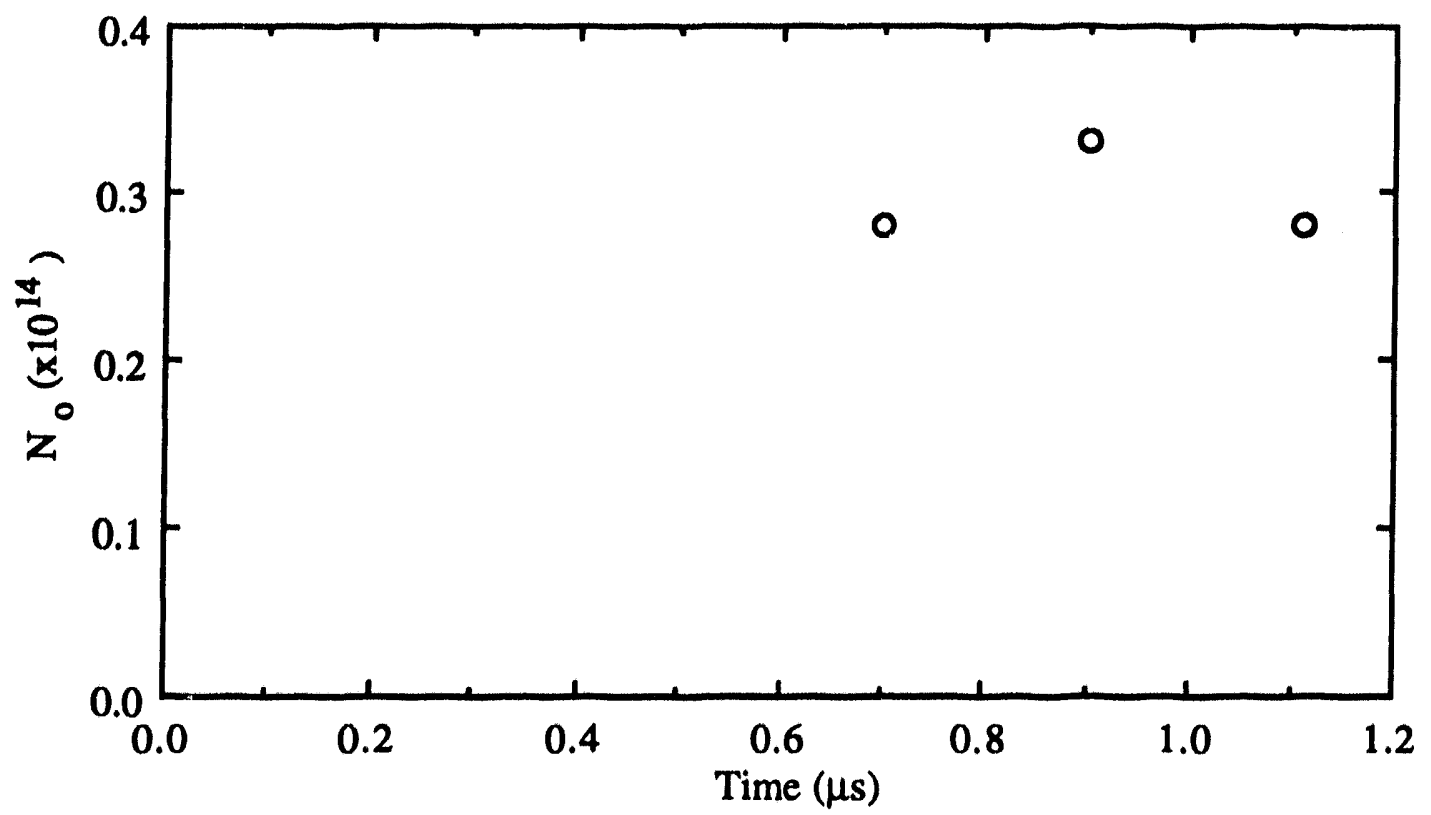

Fig. 4.7(a) Total number of alumunim neutral atoms, $N_{0}$, versus time in vacuum.

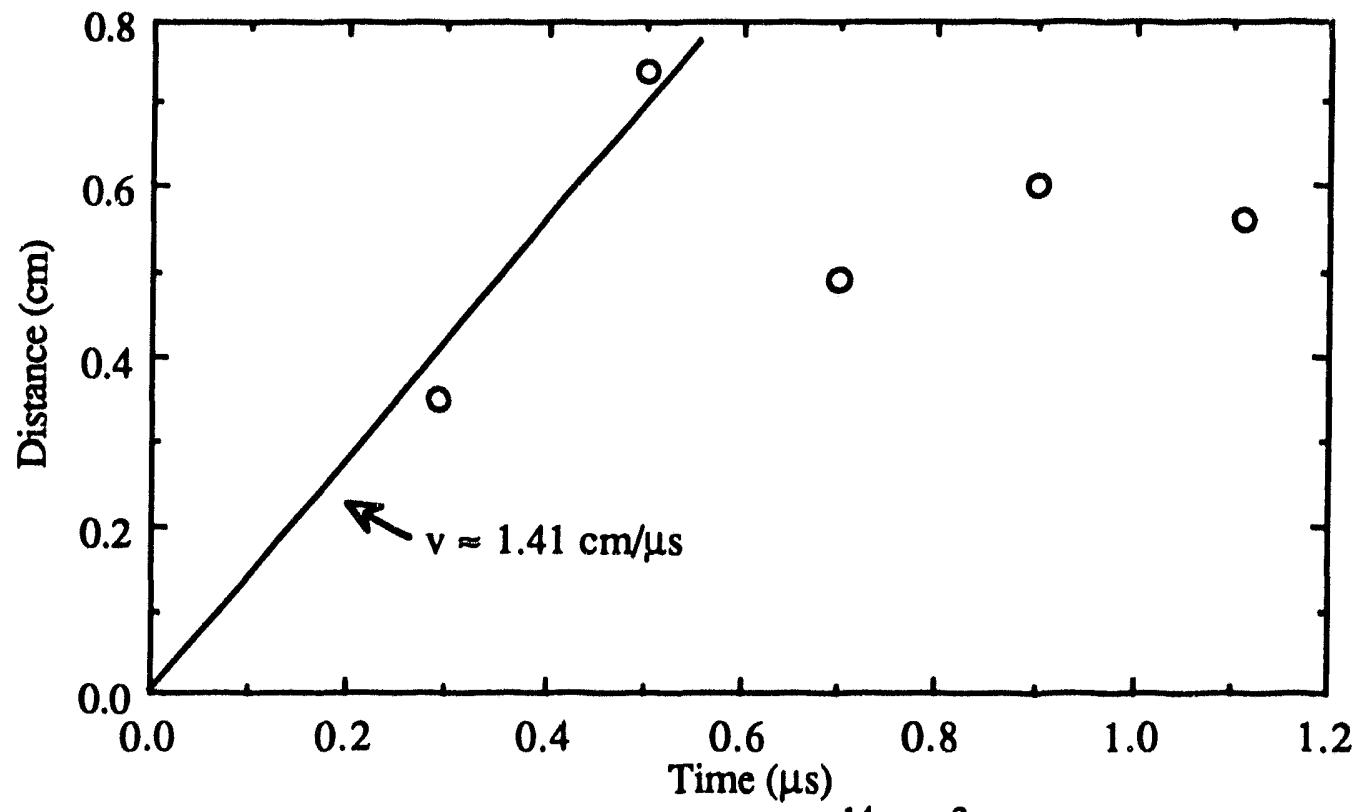

Fig. 4.7(b) Expansion of the $1 \times 10^{14} \mathrm{~cm}^{-2}$ equicontour line in vacuum. 
Figure 4.7(a) shows the total number of aluminum neutral particles on the hologram, $\mathrm{N}_{0}$, as a function of time for the vacuum time scan tracings presented in Fig. 4.6. The parameter $\mathrm{N}_{\mathrm{O}}$ is determined by tracing the holograms onto fine graph paper and counting the squares in each region. For example, the number of squares between 0.5 fringe shift and 1.0 fringe shift is counted, converted into an area in $\mathrm{cm}^{2}$, and multiplied by the calculated aluminum neutral line-density for the 0.5 fringe shift equicontour aluminum neutral line to obtain an estimate of the total number of particles represented between the 0.5 and 1.0 fringe shifts. This process is repeated for each section of the hologram and summed to approximate $\mathrm{N}_{0}$. The value $\mathrm{N}_{0}$ determined for the holograms at $0.70 \mu \mathrm{s}, 0.90 \mu \mathrm{s}$, and $1.11 \mu \mathrm{s}$ is essentially the same, $3 \times 10^{13}$, and shows consistency with time even when the peak line density decreases from $2.5 \times 10^{14} \mathrm{~cm}^{-2}$ to $1.4 \times 10^{14} \mathrm{~cm}^{-2}$.

Figure 4.7(b) plots the farthest extent of the $1 \times 10^{14} \mathrm{~cm}^{-2}$ equicontour line as a function of time for the holograms in Fig. 4.6. The expansion velocity, $v$, was determined by calculating the best fit to a line passing through the first four points as well as the origin. The expansion velocity of $1.41 \mathrm{~cm} / \mu \mathrm{s}$ is consistent with other recent measurements of the TOF most probable expansion velocity (Geo89, Wan91, Zhe89) and the leading edge expansion velocity (Dre91, Ven92a, Wal86) of laser ablation expansion in vacuum.

Figure 4.8 (a) through (1) shows another series of holograms in vacuum ( $0.2 \mathrm{mT}$ air), except this time for a laser ablative deposition configuration in which the laser is incident on the aluminum target with an angle of $\approx 690$ and an amorphous-silicon-coated silicon wafer has been placed $1.5 \mathrm{~cm}$ away from the aluminum target. The expanded dye laser 


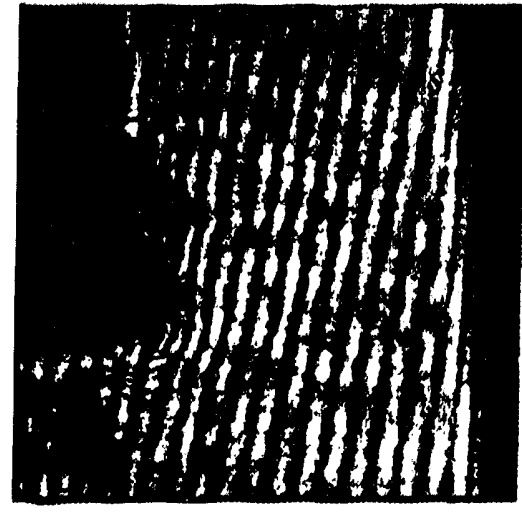

(a)

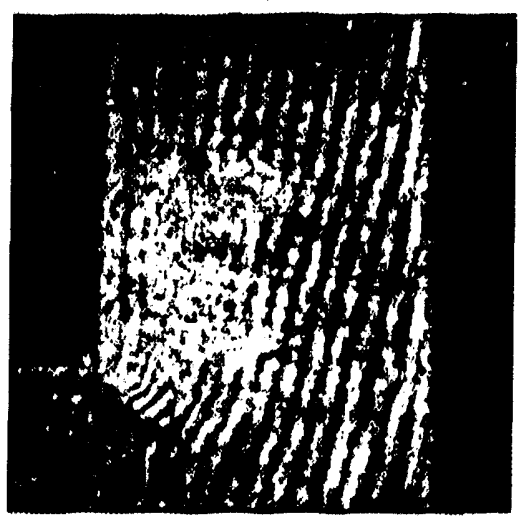

(b)

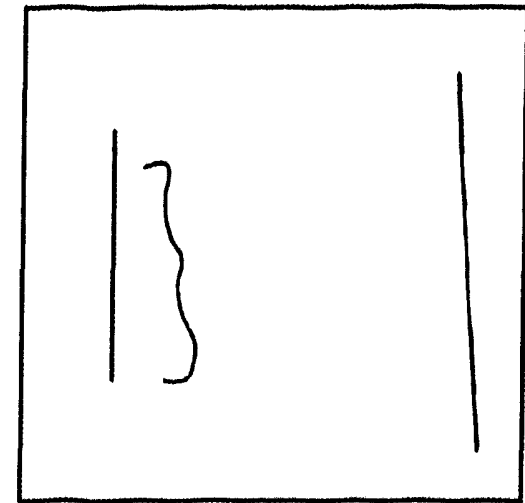

(c)

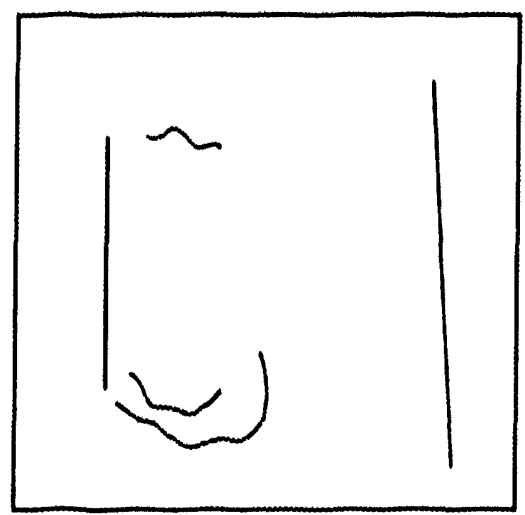

(d)

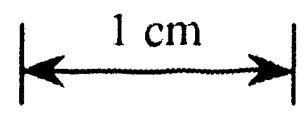

Fig. 4.8 Deposition time scan in vacuum. Hologram (a) taken at $\left(0.25 \mu_{3},-0.016 \mathrm{~nm}, \approx 5.4 \mathrm{~J} / \mathrm{cm}^{2}\right)$ and hologram (b) taken at $(0.52 \mu \mathrm{s}$, $-0.009 \mathrm{~nm}, \approx 6.2 \mathrm{~J} / \mathrm{cm}^{2}$ ). An amorphous silicon wafer at room temperature is mounted $1.5 \mathrm{~cm}$ away from the aluminum target and is on the right side in the above holograms. (c) is an equicontour plot of (a), line corresponds to $\left(0.5 \mathrm{FS}=2.0 \times 10^{14} \mathrm{~cm}^{-2}\right)$; (d) is an equicontour plot of (b), lines correspond to increasing fringe shifts, from outer to inner line: $\left(0.5 \mathrm{FS}=1.1 \mathrm{x}^{-14} \mathrm{~cm}^{-2}\right)$ and $\left(1.0 \mathrm{FS}=2.2 \times 10^{14}\right.$ $\mathrm{cm}^{-2}$ ). (From laser spot \#9 of Fig. 3.2( ,.) 


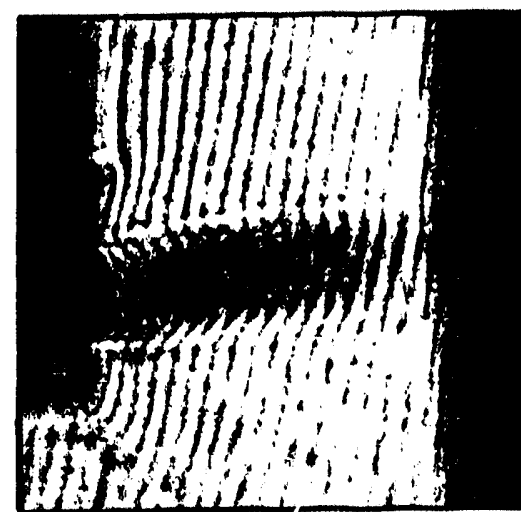

(e)

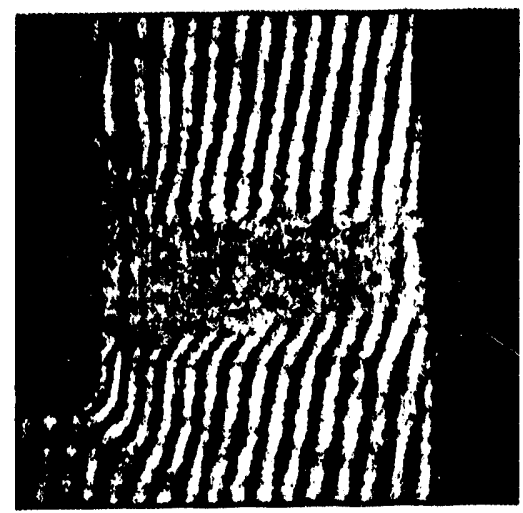

(f)

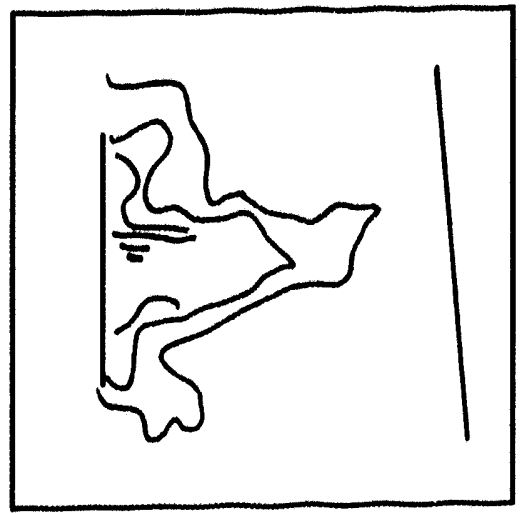

(g)

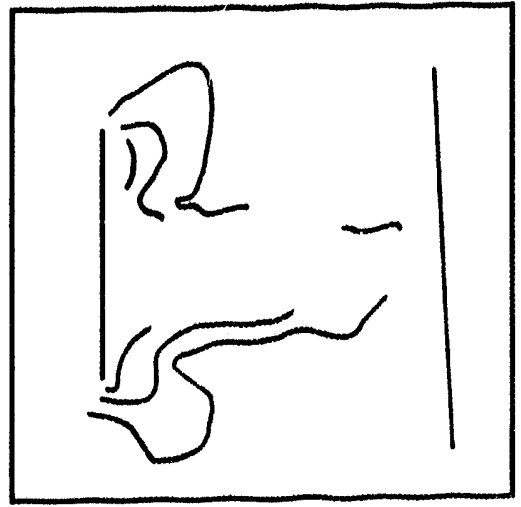

(h)

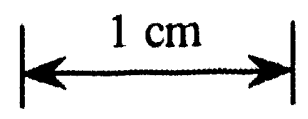

Fig. 4.8 Deposition time scan in vacuum, continued. Hologram (e) taken at $\left(1.01 \mu \mathrm{s},-0.009 \mathrm{~nm}, \approx 5.6 \mathrm{~J} / \mathrm{cm}^{2}\right)$ and hologram (f) taken at $\left(1.21 \mu \mathrm{s},-0.016 \mathrm{~nm}, \approx 6.2 \mathrm{~J} / \mathrm{cm}^{2}\right)$. An amorphous silicon wafer at room temperature is mounted $1.5 \mathrm{~cm}$ away from the aluminum target and is on the right side in the above holograms. $(\mathrm{g})$ is an equicontour plot of (e), lines correspond to increasing fringe shifts, from outer to inner line: $\left(0.5 \mathrm{FS}=1.1 \times 10^{14} \mathrm{~cm}^{-2}\right),\left(1.0 \mathrm{FS}=2.2 \times 10^{14} \mathrm{~cm}^{-2}\right),(1.5 \mathrm{FS}$ $\left.=3.3 \times 10^{14} \mathrm{~cm}^{-2}\right),\left(2.0 \mathrm{FS}=4.4 \times 10^{14} \mathrm{~cm}^{-2}\right),\left(2.5 \mathrm{FS}=5.5 \times 10^{14} \mathrm{~cm}^{-2}\right)$, and $\left(3.0 \mathrm{FS}=\right.$ and $\left.6.6 \times 10^{14} \mathrm{~cm}^{-2}\right)$; (h) is an equicontour plot of (f), lines correspond to increasing fringe shifts, from outer to inner line: $\left(0.5 \mathrm{FS}=2.0 \times 10^{14} \mathrm{~cm}^{-2}\right),\left(1.0 \mathrm{FS}=4.0 \times 10^{14} \mathrm{~cm}^{-2}\right)$, and $(1.5 \mathrm{FS}$ $=5.9 \times 10^{14} \mathrm{~cm}^{-2}$ ). (From laser spot \#9 in Fig. 3.2(b).) 


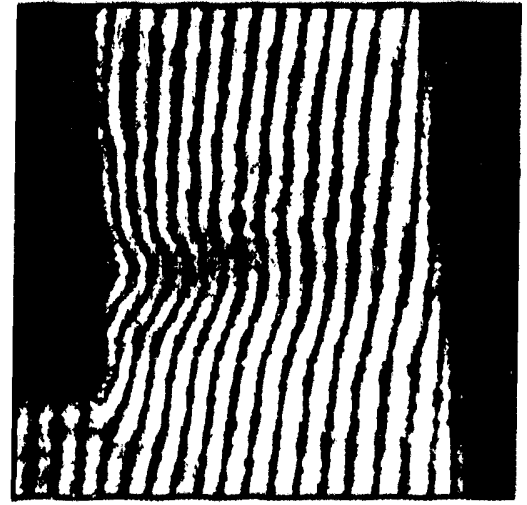

(i)

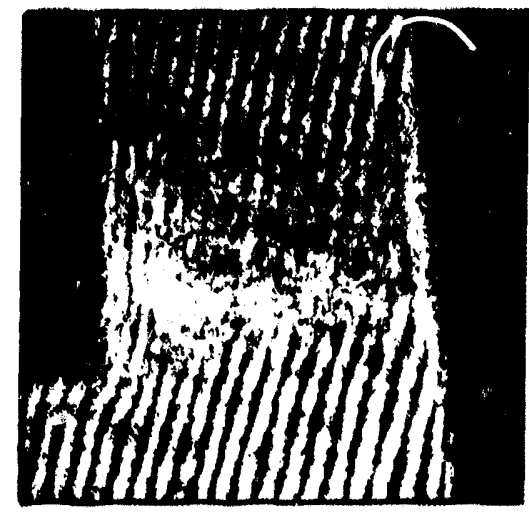

(j)

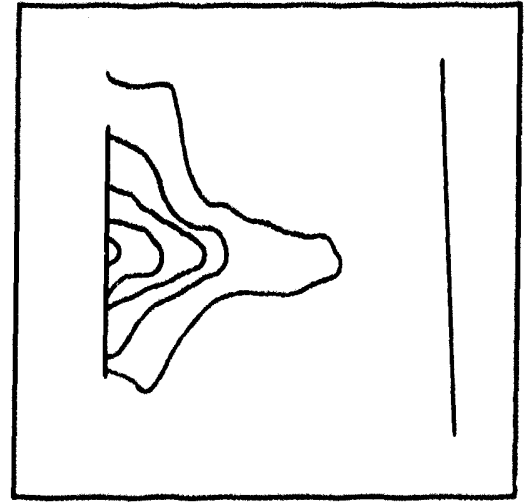

(k)

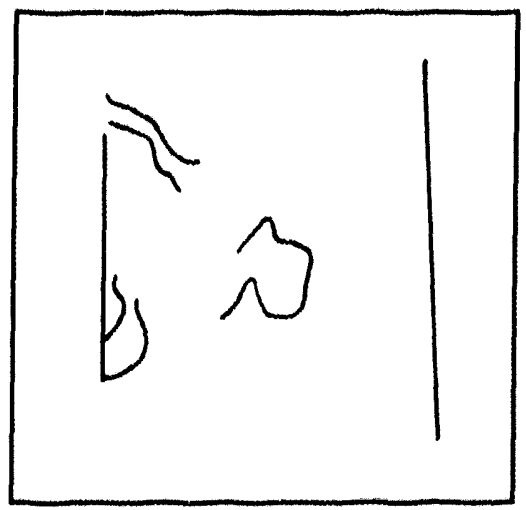

(1)

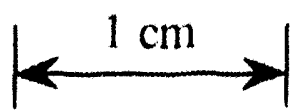

Fig. 4.8 Deposition time scan in vacuum, continued. Hologram (i) taken at $\left(1.69 \mu \mathrm{s},-0.009 \mathrm{~nm}, \approx 6.2 \mathrm{~J} / \mathrm{cm}^{2}\right)$ and hologram (j) taken at $\left(2.03 \mu \mathrm{s},-0.003 \mathrm{~nm}, \approx 6.2 \mathrm{~J} / \mathrm{cm}^{2}\right)$. An amorphous silicon wafer at room temperature is mounted $1.5 \mathrm{~cm}$ away from the aluminum target and is on the right side in the above holograms. (k) is an equicontour plot of (i), lines correspond to increasing fringe shifts, from outer to inner line: $\left(0.5 \mathrm{FS}=1.1 \times 10^{14} \mathrm{~cm}^{-2}\right),\left(1.0 \mathrm{FS}=2.2 \times 10^{14} \mathrm{~cm}^{-2}\right),(1.5 \mathrm{FS}$ $\left.=3.3 \times 10^{14} \mathrm{~cm}^{-2}\right),\left(2.0 \mathrm{FS}=4.4 \times 10^{14} \mathrm{~cm}^{-2}\right)$, and $\left(2.5 \mathrm{FS}=5.5 \times 10^{14}\right.$ $\left.\mathrm{cm}^{-2}\right)$; (l) is an equicontour plot of $(\mathrm{j})$, lines correspond to increasing fringe shifts, from outer to inner line: $\left(0.5 \mathrm{FS}=3.2 \times 10^{13} \mathrm{~cm}^{-2}\right)$ and $\left(1.0 \mathrm{FS}=6.9 \times 10^{13} \mathrm{~cm}^{-2}\right.$ ). (From laser spot \#9 of Fig. 3.2(b).) 


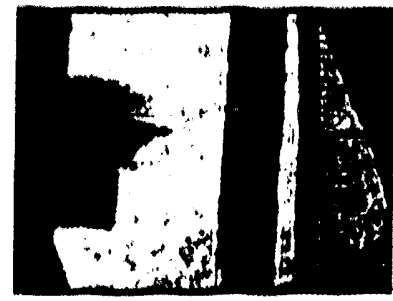

(m)

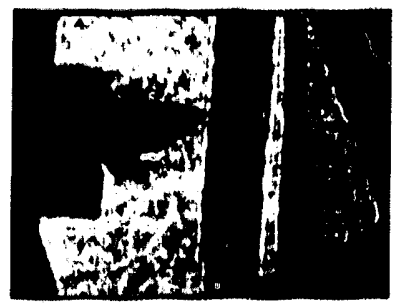

(n)

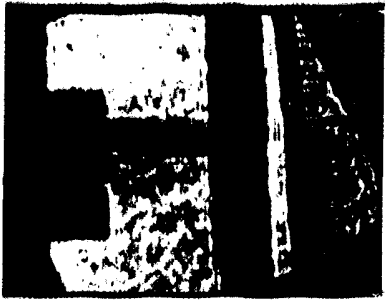

(o)

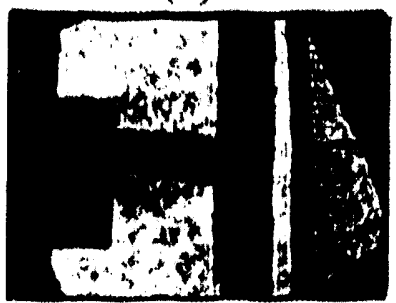

(p)

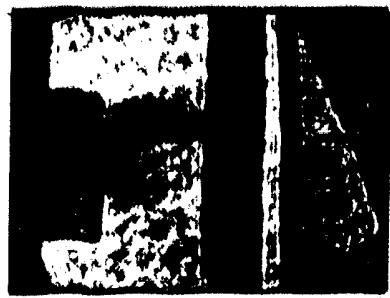

(q)

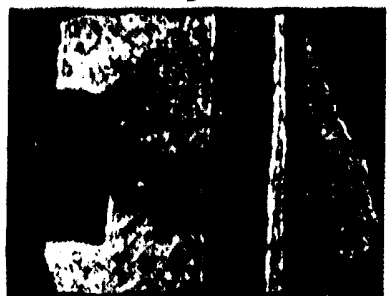

(r)

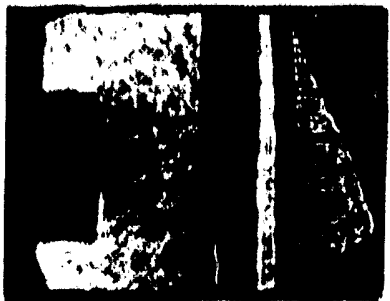

(s)

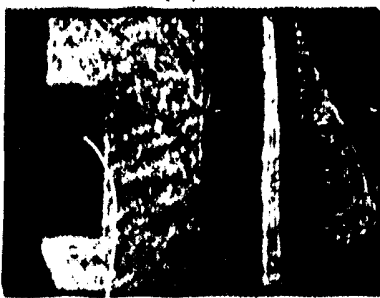

(t)

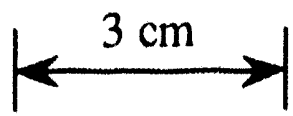

Fig. 4.8 Deposition time scan in vacuum, continued. Dye Laser Resonant Absorption Photographs (DLRAP's) that correspond to, but are not simultaneous to, holograms (a), (b), (e), (f), (i) and (j), plus two additional DLRAP's at later times: $[(\mathrm{m}), 0.21 \mu \mathrm{s},-0.016 \mathrm{~nm}, \approx 5.4$ $\left.\mathrm{J} / \mathrm{cm}^{2}\right],\left[(\mathrm{n}), 0.50 \mu \mathrm{s},-0.009 \mathrm{~nm}, \approx 5.9 \mathrm{~J} / \mathrm{cm}^{2}\right],[(0), 1.00 \mu \mathrm{s},-0.009 \mathrm{~nm}$, $\left.\approx 5.8 \mathrm{~J} / \mathrm{cm}^{2}\right],\left[(\mathrm{p}), 1.21 \mu \mathrm{s},-0.009 \mathrm{~nm}, \approx 5.4 \mathrm{~J} / \mathrm{cm}^{2}\right.$ ], [(q), $1.61 \mu \mathrm{s},-0.009$ $\left.\mathrm{nm}, \approx 5.4 \mathrm{~J} / \mathrm{cm}^{2}\right],\left[(\mathrm{r}), 2.00 \mu \mathrm{s},-0.003 \mathrm{~nm}, \approx 5.4 \mathrm{~J} / \mathrm{cm}^{2}\right],[(\mathrm{s}), 4.02 \mu \mathrm{s}$, $\left.-0.003 \mathrm{~nm}, \approx 5.8 \mathrm{~J} / \mathrm{cm}^{2}\right]$, and [(t), $\left.5.02 \mu \mathrm{s},-0.003 \mathrm{~nm}, \approx 4.8 \mathrm{~J} / \mathrm{cm}^{2}\right]$. Note the transparent ruler to the right side of the silicon wafer and the wafe, holder, and that the DLRAP image is inverted with respect to the holograms. (From laser spot \#9 in Fig. 3.2(b).) 
beam was not perfectly parallel to the target and wafer surfaces for this experimental run, so the separation distance appears to be only $\approx 1.25 \mathrm{~cm}$.

The ablation laser energy was higher for this run, so the fluence is higher for Fig. 4.8 than for Fig. 4.6, even though the target is tilted and the spot size is larger. The ablation plume begins clcse to the surface and expands toward the silicon wafer at earlier times $(0.25 \mu \mathrm{s}, 0.52 \mu \mathrm{s}$, and $1.00 \mu \mathrm{s})$. By $1.21 \mu \mathrm{s}$, the ablation plume makes contact with the wafer and then by $1.69 \mu \mathrm{s}$, the plume is observed to begin receding back to the aluminum surface. This does not mean that the aluminum neutrals flow toward the wafer and then recede back as an ocean wave advances and recedes on a beach, rather the aluminum neutrals are continually expanding outward and the RHI-detectable portion of the aluminum neutral atoms appears to extend from and recede back to the aluminum target surface. The peak line-density $\left(>6 \times 10^{14} \mathrm{~cm}^{-2}\right)$ appears to remain at the target surface, although the holograms are greatly obscured by absorption until $1.69 \mu \mathrm{s}$. The inferred particle density is estimated to be $>5 \times 10^{14} \mathrm{~cm}^{-3}$.

Figure $4.8(\mathrm{~m})$ through $(\mathrm{t})$ shows a series of Dye-Laser-ResonanceAbsorption-Photographs (DLRAP's) that correspond to the holograms in Fig. 4.8. During the experimental run, four holograms were taken and then the corresponding four DLRAP's were taken under the same conditions. Here also, the ablation plume is seen to expand toward the wafer at earlier times $(0.21 \mu \mathrm{s})$, make contact with the wafer at intermediate times $(0.50 \mu \mathrm{s}, 1.00 \mu \mathrm{s}, 1.21 \mu \mathrm{s}$, and $1.61 \mu \mathrm{s})$, and recede from the wafer at later times $(2.00 \mu \mathrm{s}$, and $4.02 \mu \mathrm{s})$. By $5.02 \mu \mathrm{s}$, the ablation plume is no longer observable. Note that DLRAP is more 
sensitive than RHI, and thus the presence of the ablation plume was detected as late as $4.02 \mu \mathrm{s}$ with DLRAP.

The total number of aluminum neutral atoms at $1.69 \mu \mathrm{s}$ was $8.3 \times 10^{13} \mathrm{~cm}^{-2}$; the parameter $N_{0}$ could only be determined for one hologram (Fig. 4.8(i)) due to the absorption observed in this time scan. Since this hologram, at $1.69 \mu \mathrm{s}$, was taken after the plume appeared to begin to recede back to the target surface, its measurement of $N_{0}$ is perhaps four times lower than the true number of aluminum neutral particles leaving the target surface (as estimated from Fig. $4.8(\mathrm{~g})$, which makes $\approx 3 \times 10^{14}$ aluminum atoms total). When compared with the first time scan in vacuum, the plume expansion in Fig. 4.8 is much more directional, and thus a greater number of particles ( $>3$ times) expanding in a more forward direction produce absorption for a greater distance away from the target, even for larger $|\Delta \lambda|$ 's. The variation in plume expansion shape has been observed to depend upon experimental parameters such as fluence magnitude, fluence uniformity, background pressure, and the distance from the laser focal lens to the target (Sap92, Lin93). Therefore, the plume shapes presented here vary from run-torun as well as from shot-to-shot, as shown in Fig. 4.4.

Figure 4.9 shows the expansion of the $1 \times 10^{14} \mathrm{~cm}^{-2}$ equicontour line as a function of time for the vacuum-deposition holograms. The leading edge expansion velocity of $\approx 1.05 \mathrm{~cm} / \mu \mathrm{s}$ is about the same as the first time scan in vacuum, $\approx 1.41 \mathrm{~cm} / \mu \mathrm{s}$.

Figure 4.10 shows a photograph of the amorphous-silicon-coated silicon wafer with the deposited aluminum thin film after 55 ablation pulses on spot \#9 of Fig. 3.2(b), and Figure 4.11 shows a rendition of the deposition spot with an overlay of the laser ablation spot. Note the 


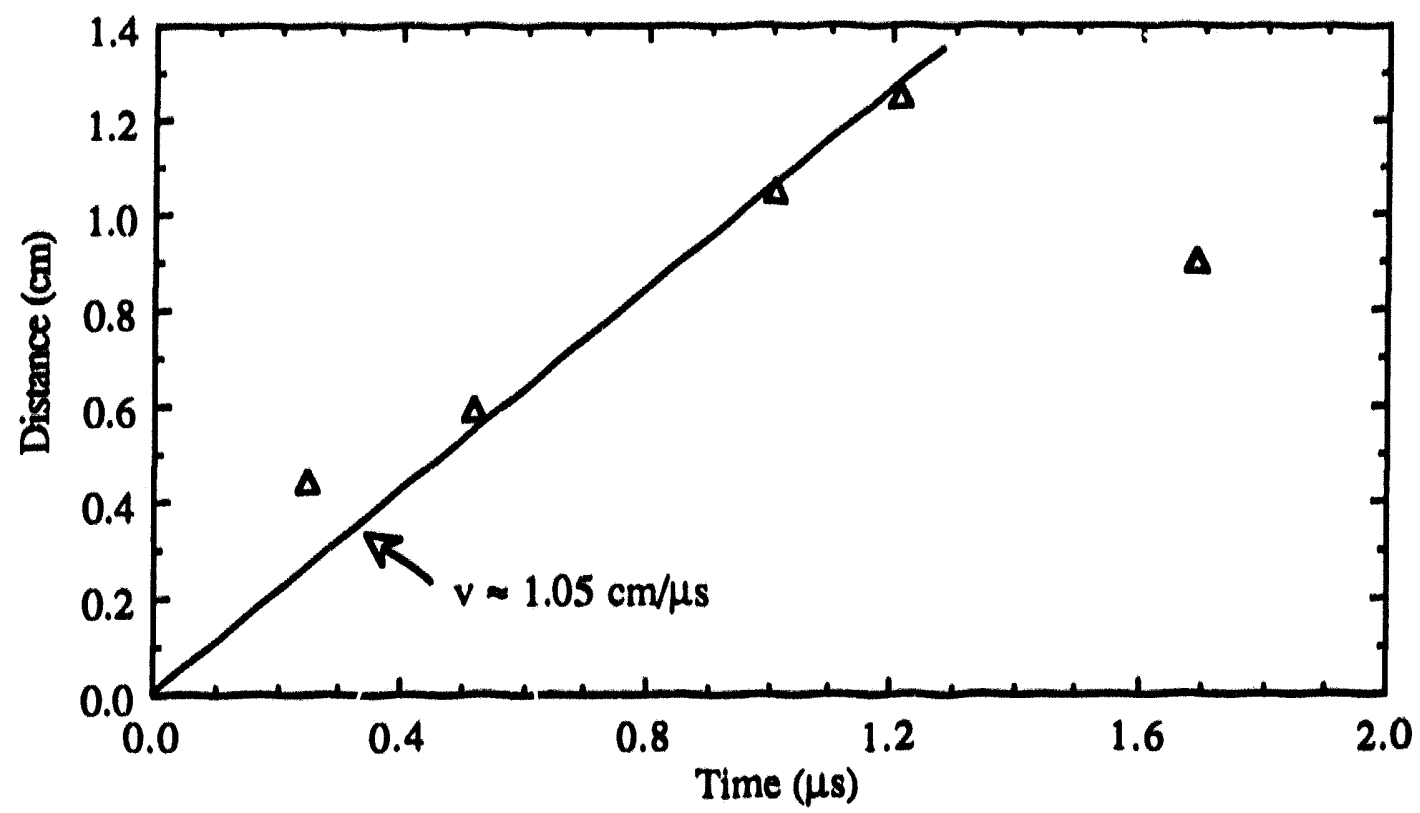

Fig. 4.9 Expansion of the $1 \times 10^{14} \mathrm{~cm}^{-2}$ equicontour line in vacuum for the deposition case.

photographic orientations: up in Fig. 4.11 is to the right in Fig. 4.10, upward in the DLRAP's of Fig. 4.8 and downward in the holograms of Fig. 4.8. Figure 4.11 shows clearly that the ablation plume expanded differentially in the $x$ - and $y$-direction ( $z$ defined as perpendicular to the target and substrate surfaces), and that it expanded more quickly in the direction corresponding to the narrow width of the ablation spot. This sideways motion has been previously observed in atmospheric pressure air and modeled using hydrodynamic equations (Mio92); i.e., the expansion of the ablation plume particles in the sideways direction is governed primarily by self-collision and thus self-diffusion mechanisms $\Gamma=-D \nabla n$, especially for expansion into vacuum and lower pressure background gases. For an initially $(<0.1 \mu \mathrm{s})$ uniform particle density, the more narrow dimension of the laser spot corresponds to a higher density gradient and thus a higher expansion velocity. Thus a circular ablation 


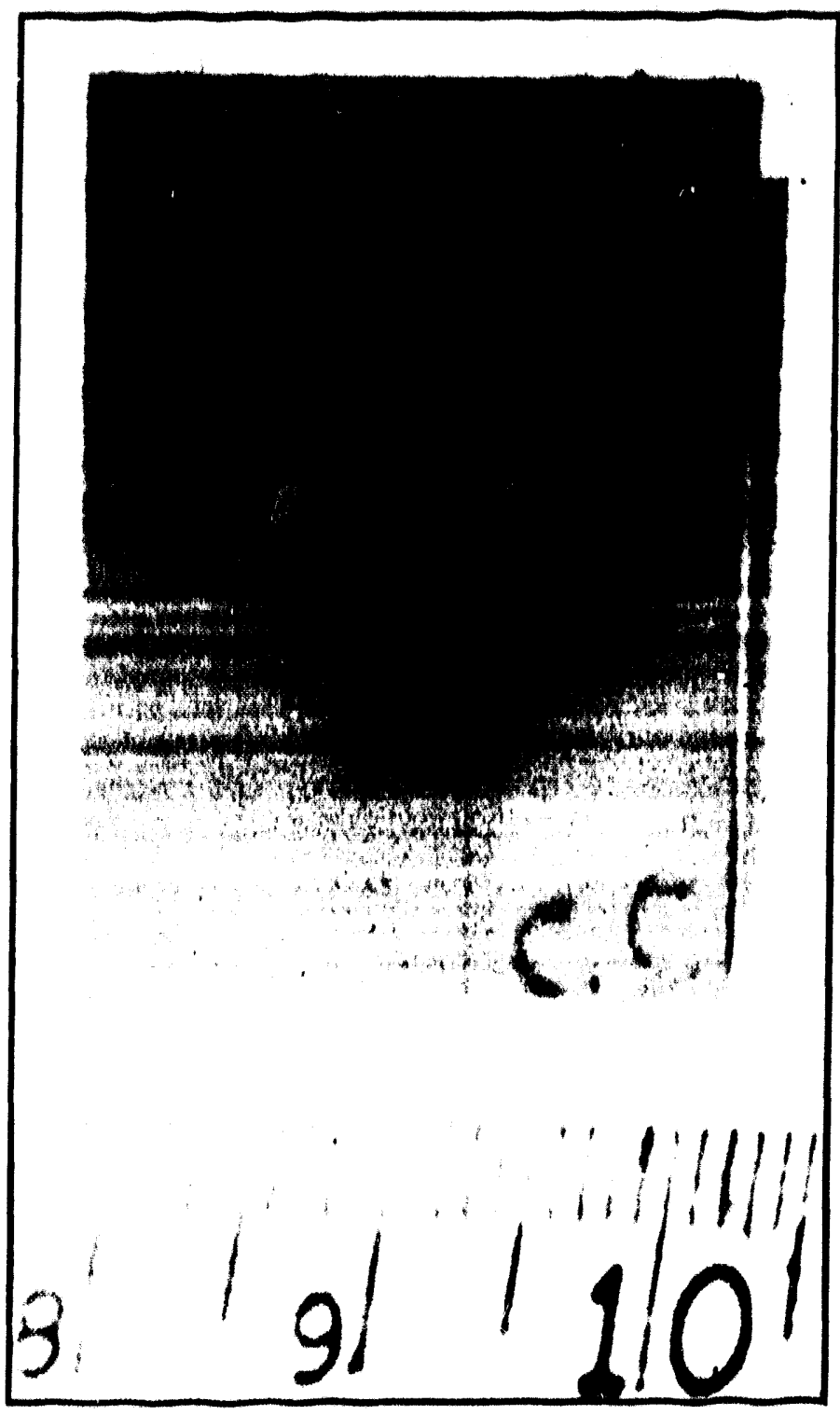

Tig. 4.10 Laser ablation deposition substrate (amorphous silicon) after $55 \mathrm{KrF}$ ablation laser pulses on a $99.8 \%$ pure aluminum target (spot \#9 of Fig. 3.2(b)). Target-substrate separation distance was $1.5 \mathrm{~cm}$; substrate was at room temperature. 


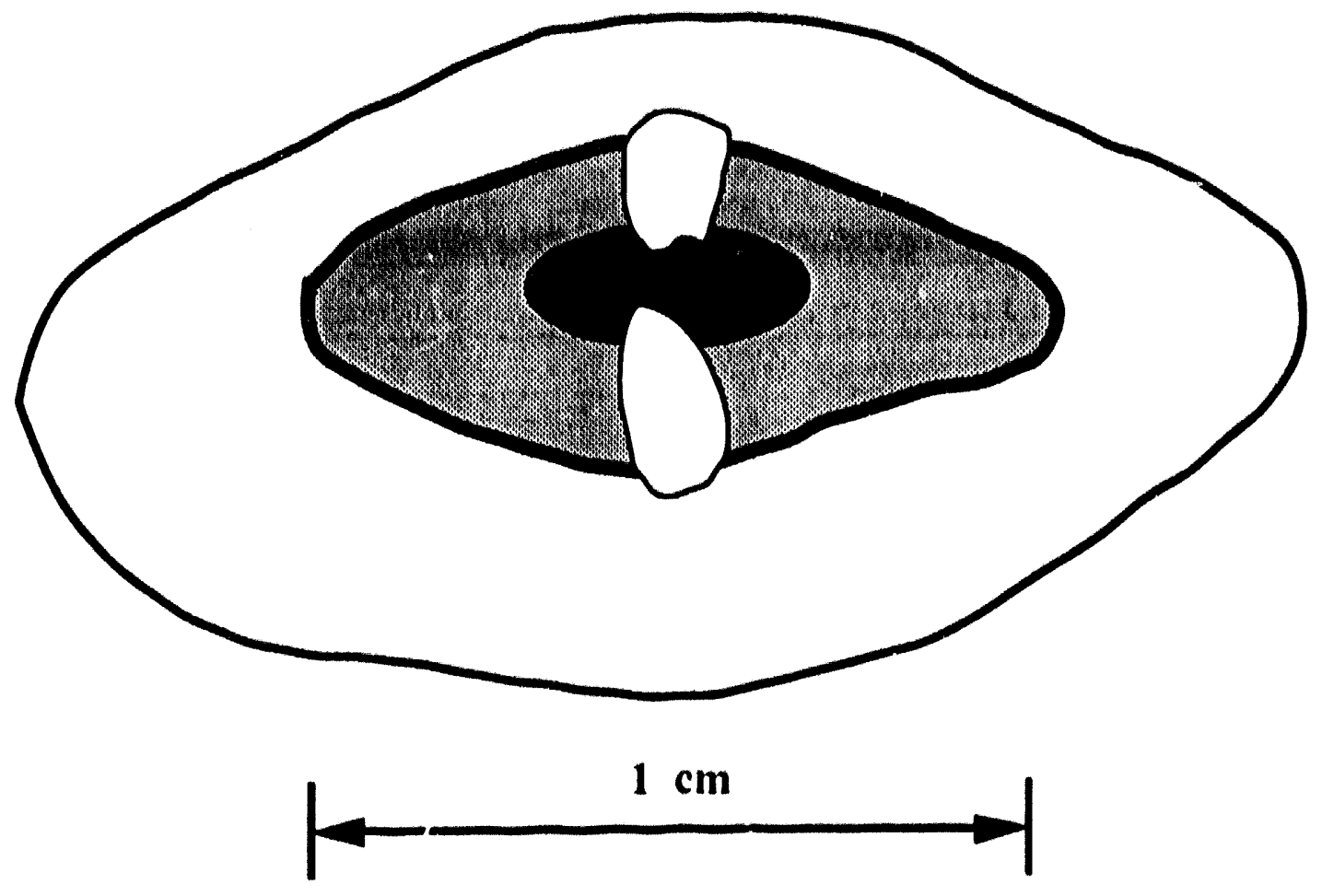

Fig. 4.11 The laser ablation spot from the target (spot \#9 of Fig. 3.2(b), here shown as white) overlaying a depiction of the aluminum thin film deposited on the amorphous silicon substrate (from Fig. 4.10, here shown as various lines and shades) in the original orientation. Note that the direction of greater lateral plume expansion corresponds to the narrower dimension of the ablation spot.

spot would produce a circular thin film (in vacuum and lower background pressures) while a (double spot) rectangular ablation spot, as shown here, produces an oval thin film with the largei dimension turned $90^{\circ}$ with respect to the longer ablation spot dimension (Mio92).

The shape of the thin film in Fig. 4.11 is therefore an indication that aluminum-aluminum collisions play a significant role in the expansion of the ablation plumes studied here. It is also a confirmation of the previously utilized assumption that the ablation plume can be 
considered to be Maxwellian in distribution from the collisions at early times, and in quasi-equilibrium over the duration of the dye laser pulse (20 ns). This is not to say that a significant percentage of ablated particles are experiencing collisions at the same time the hologram is taken (e.g., $0.2-2.0 \mu \mathrm{s}$ ), but rather the collisions the ablated particles experience at very early times, when the ablation plume has a high enough particle density, determine and "fix" the velocity distribution that is observed at later times when collisions are no longer significant.

It has been calculated using a Monte Carlo simulation that a quasiMaxwelliam velocity distribution is obtained for a laser ablation plume if the average number of collisions per ablated particle is greater than $m 3$, which is based on the ablative removal of 0.8 monolayers per pulse for the specific case investigated (Noo87). It has therefore been suggested that it would be difficult to produce an ablation plume whose particles experimence less than 3 collisions on average and thus not have a quasiMaxwellian velocity distribution (Kel88).

The width of the central, two thin film regions in Fig. 4.11 (inside the thick line), as viewed by the dye laser (left to right), is approximately the same width as the ablation plume in Fig. 4.8(e). The thickness of this region can be approximately calculated by assuming that most of the deposited particles are in this region, that the film has a diamond-basedpyramid shape, and by assuming that the total number of aluminum atoms is $\approx 3 \times 10^{14}$ (from above). Since the density of solid aluminum is $6.02 \times 10^{22} \mathrm{~cm}^{-3}$ and since the thin film was made with 55 ablation pulses, the estimated maximum heigh of the thin-film-pyramid is calculated to be $\approx 35 \mathrm{~nm}$, assuming $100 \%$ of the $\mathrm{Al}$ neutrals impinge upon the surface and stick. 


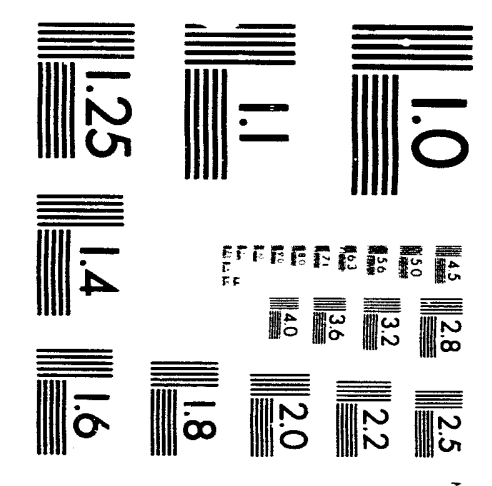



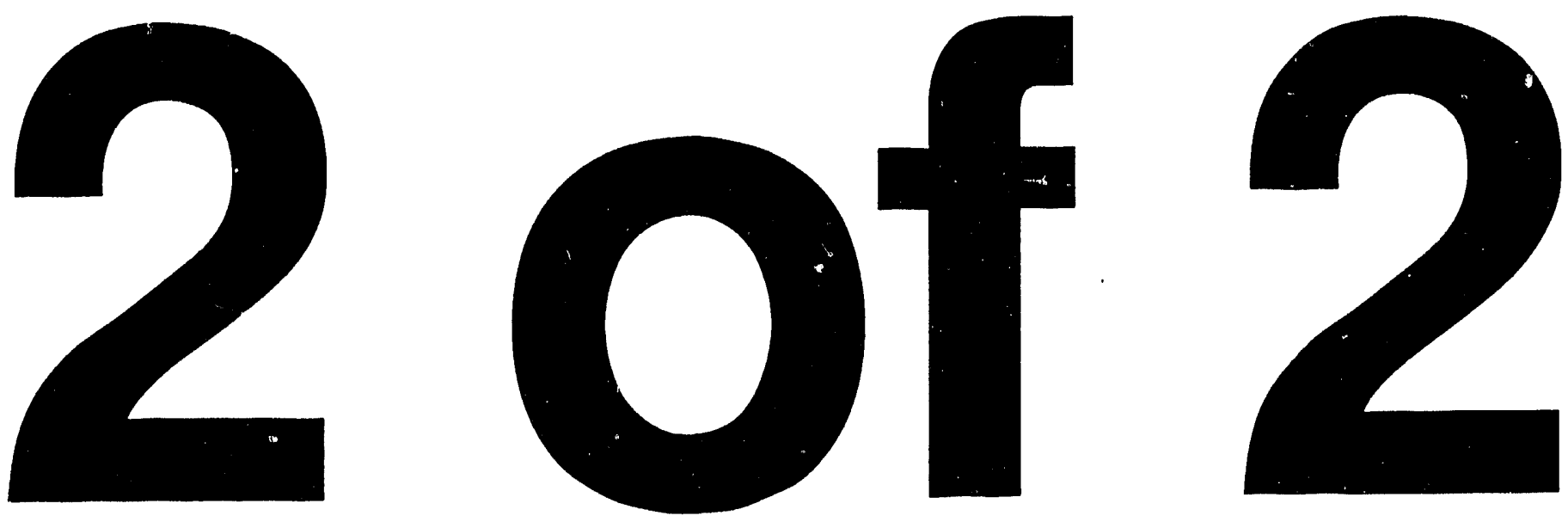
The wafer that was used in this experiment was crystalline silicon $\left(\approx 500 \mu \mathrm{m}\right.$ thick) over which a $115 \mathrm{~nm}$ coating of $\mathrm{SiO}_{2}$ was deposited, followed by a $493 \mathrm{~nm}$ layer of amorphous silicon (Pen93). The aluminum thin film was deposited onto the amorphous silicon layer. Attempts to measure the aluminum thin film thickness using the four point resistance probe method (Pen93) were unsatisfactory. The thin film appeared to be non-conductive, perhaps indicating an oxide layer had formed on the thin film. Also, the morphology of the thin film was unknown, making it difficult to use a tabulated value of resistivity in order to calculate the thin film thickness.

A zeroeth order estimate of the target ablation rate can be made using the measured spot size of $0.049 \mathrm{~cm}^{2}$ for the ablation spot on Fig. 4.11. The ablation rate of $3 \times 10^{14}$ aluminum atoms per pulse corresponds to a surface etching rate of $1 \mathrm{~nm} / \mathrm{pulse}$, which is close to the etching rate of $5-10 \mathrm{~nm} /$ pulse measured by Kelly and Rothenburg for $2.3 \mathrm{~J} / \mathrm{cm}^{2}$ ablation of aluminum (Kel85). Again, nearly half of the ablated atoms may be redeposited on the target.

A zeroeth order estimate of the amount of energy absorbed by the target per ablated particle can be made. With up to $300 \mathrm{~mJ}$ reaching the target surface (for Fig. 4.8) and a reflectivity of $92.5 \%$, the aluminum target absorbed $22.5 \mathrm{~mJ}$ total energy to thermally ablate (expel) $\approx 3 \times 10^{14}$ particles, which is $\approx 466 \mathrm{eV}$ absorbed by the target per ablated particle. Since the kinetic energy of each particle is on the order of $1 \mathrm{eV}$, almost all of the energy absorbed by the target remains in the target and is thermally dissipated.

Figures $4.12,4.14$, and 4.16 show the holograms and tracings for time scans in 14 mTorr, 52 mTorr, and 210 mTorr argon background 
gas, while Figures $4.13,4.15$, and 4.17 show $\mathrm{N}_{\mathrm{O}}$ and the expansion of the $1 \times 10^{14} \mathrm{~cm}^{-2}$ equicontour for each of the holograms. As in the vacuum cases, the ablation plume begins near the target at earlier times and the peak line-density recedes back to the surface at later times. Likewise, $\mathrm{N}_{\mathrm{O}}$ and the expansion velocity are close to the values measured for the two vacuum cases. The only exception to this is for 52 mTorr argon gas, where in Fig. 4.17 (h) the minimum detectable fringe shift (0.5 FS) has completely separated itself from the target surface.

Figure 4.18 compiles the information about the five time scans presented thus far. The values of $\mathrm{N}_{\mathrm{O}}$ in Fig. 4.18(a) for the most part range from $\approx 1 \times 10^{13}$ aluminum neutral atoms to $1 \times 10^{14}$ aluminum neutral atoms. These are actually low estimates of the number of ablated particles because: 1) the step method for approximating the number of particles between each of the fringe shift equicontour lines gives low approximations, 2) the earlier-time holograms which would better represent the total number of particles often had too much absorption to make counting possible, and 3 ) only one type of particle was counted. It is again suggested that the total number of aluminum atoms ablated from the target surface (as neutrals, ions, and molecules) is in the range of $1 \times 10^{14}-1 \times 10^{15}$ for the range of fluences used here.

Figure 4.18(b) compiles the expansions of the $1 \times 10^{14} \mathrm{~cm}^{-2}$ equicontours for the five time scans. Their expansion velocities are relatively similar and have the range $1.05 \mathrm{~cm} / \mu \mathrm{s}-1.41 \mathrm{~cm} / \mu \mathrm{s}$. These expansion velocities can be used to calculate the initial temperature of the collisional ablation plume using the relation (Ven91) 


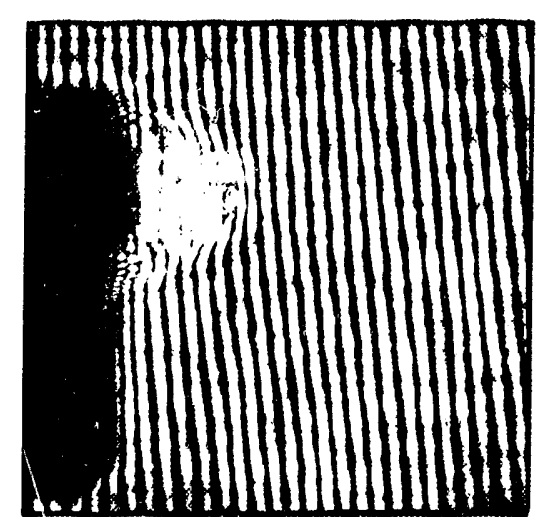

(a)

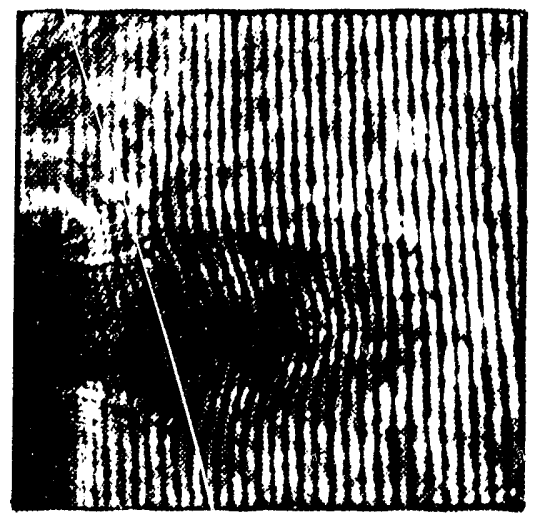

(b)

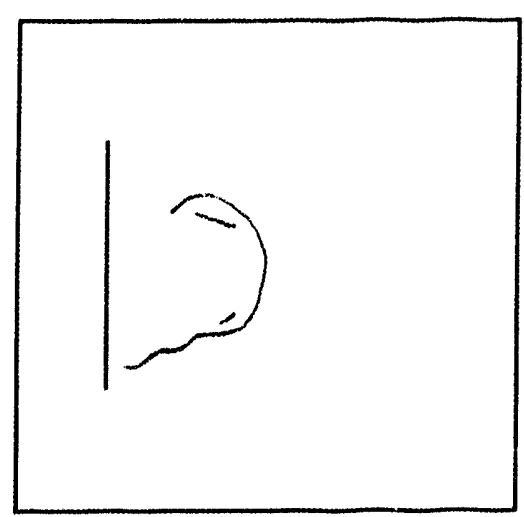

(c)

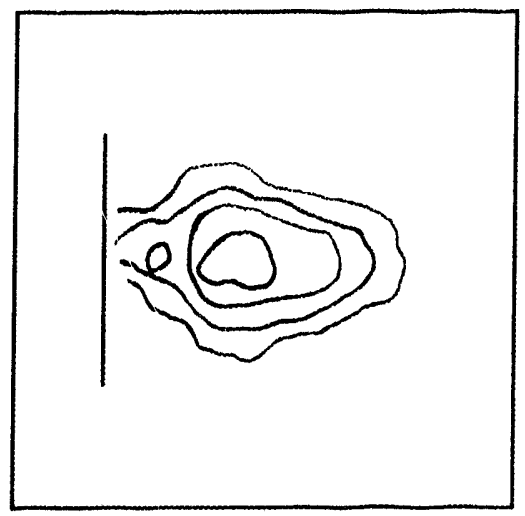

(d)

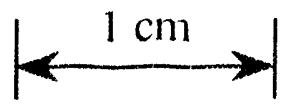

Fig. 4.12 Time scan in $14 \mathrm{~m}$ Torr argon gas. Hologram (a) taken at $\left(0.40 \mu \mathrm{s},-0.006 \mathrm{~nm}, \approx 2.4 \mathrm{~J} / \mathrm{cm}^{2}\right)$ and hologram (b) taken at $(1.00 \mu \mathrm{s}$, $-0.006 \mathrm{~nm}, \approx 2.4 \mathrm{~J} / \mathrm{cm}^{2}$ ). (c) is an equicontour plot of (a), lines correspond to increasing fringe shifts, from outer to inner line: $(0.5$ $\mathrm{FS}=7.0 \times 10^{13} \mathrm{~cm}^{-2}$ ) and $\left(1.0 \mathrm{FS}=1.4 \times 10^{14} \mathrm{~cm}^{-2}\right)$; (d) is an equicontour plot of (b), lines correspond to increasing fringe shifts, from outer to inner line: $\left(0.5 \mathrm{FS}=7.0 \times 10^{13} \mathrm{~cm}^{-2}\right),\left(1.0 \mathrm{FS}=1.4 \times 10^{14} \mathrm{~cm}^{-2}\right),(1.5 \mathrm{FS}$ $=2.1 \times 10^{14} \mathrm{~cm}^{-2}$ ), and $\left(2.0 \mathrm{FS}=2.9 \times 10^{14} \mathrm{~cm}^{-2}\right.$ ). (From laser spot \#2 of Fig. 3.2(b).) 


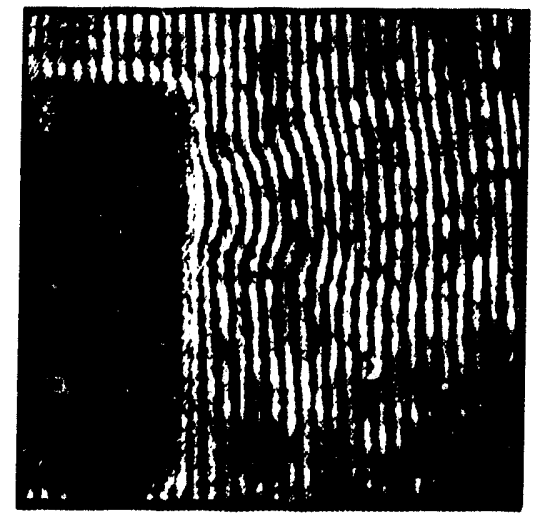

(e)

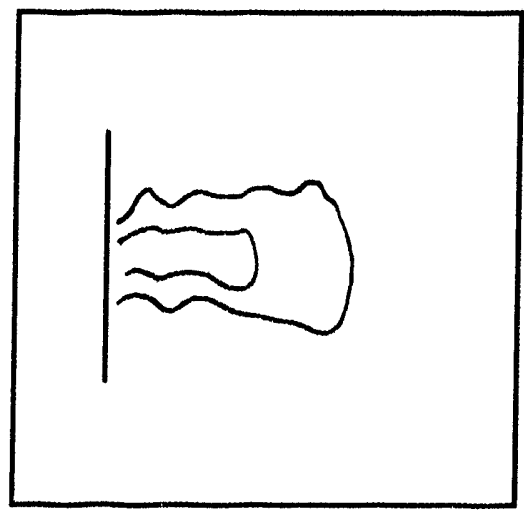

(f)

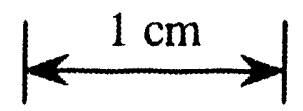

Fig. 4.12 Time scan in 14 mTorr argon gas, continued.

Hologram (e) was taken at $\left(2.00 \mu \mathrm{s},-0.006 \mathrm{~nm}, \approx 2.4 \mathrm{~J} / \mathrm{cm}^{2}\right)$. (f) is an equicontour plot of (e), lines correspond to increasing fringe shifts, from outer to inner line: $\left(0.5 \mathrm{FS}=7.0 \times 10^{13} \mathrm{~cm}^{-2}\right)$ and $\left(1.0 \mathrm{FS}=1.4 \times 10^{14} \mathrm{~cm}^{-2}\right.$ ). (From laser spot \#2 of Fig. 3.2(b).) 


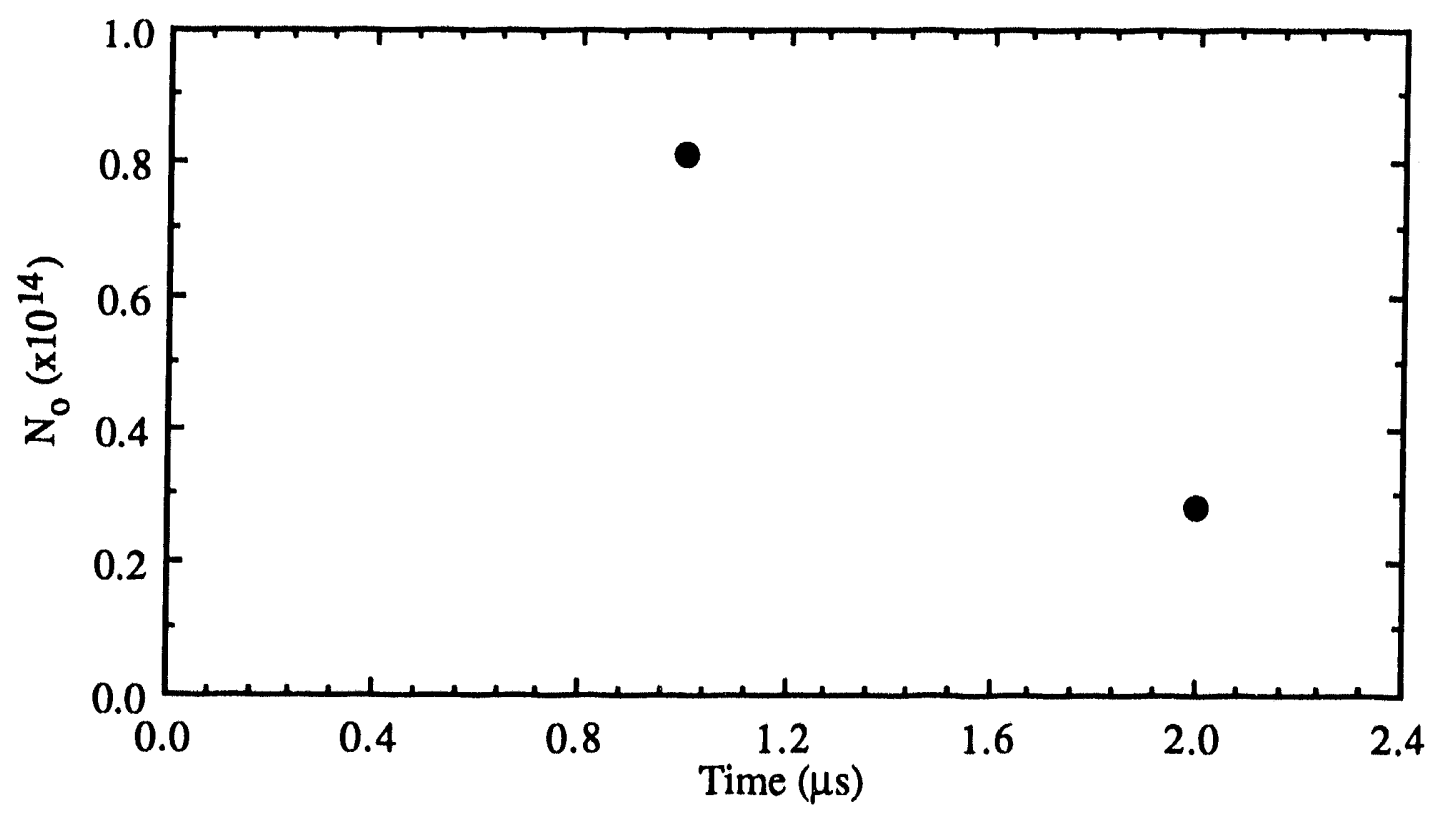

Fig. 4.13(a) Total number of aluminum neutral atoms, $N_{0}$, versus time in 14 mTorr argon gas.

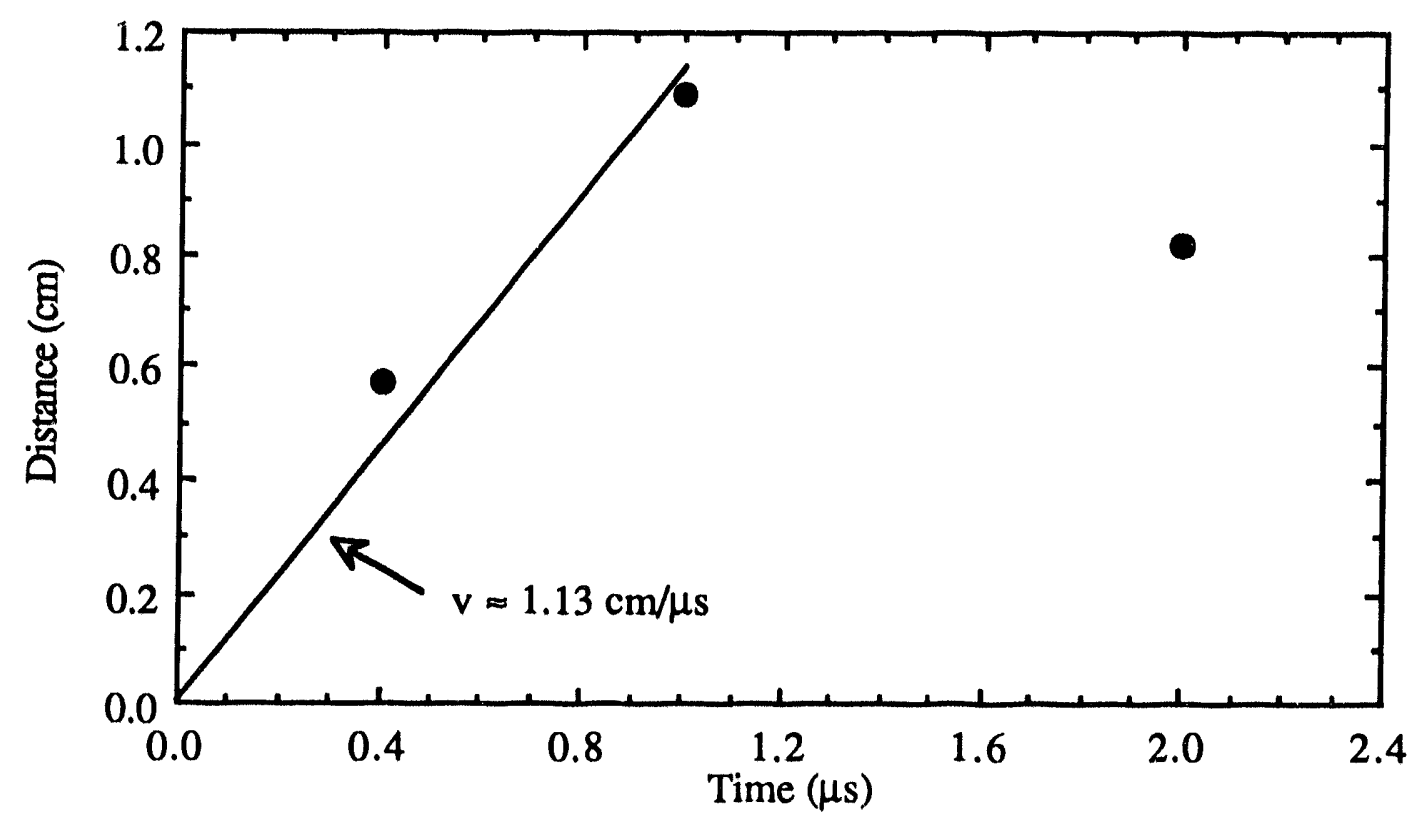

Fig. 4.13(b) Expansion of the $1 \times 10^{14} \mathrm{~cm}^{-2}$ equicontour line in $14 \mathrm{mTorr}$ argon gas. 


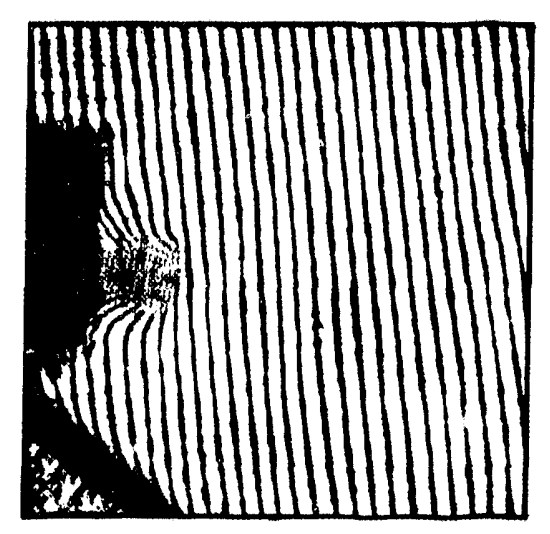

(a)

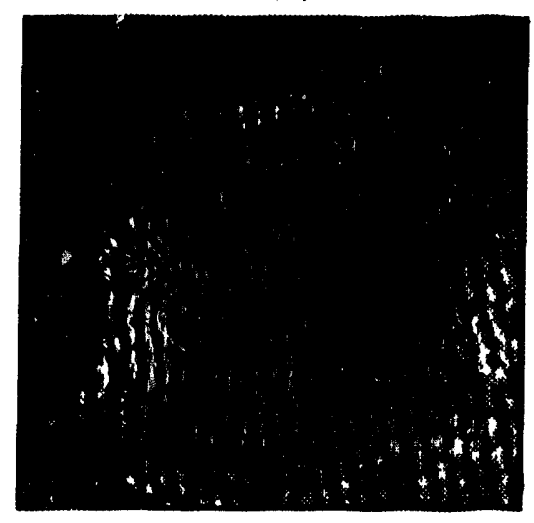

(b)

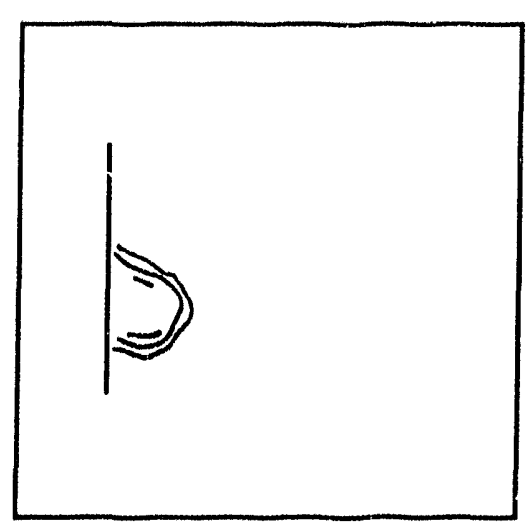

(c)

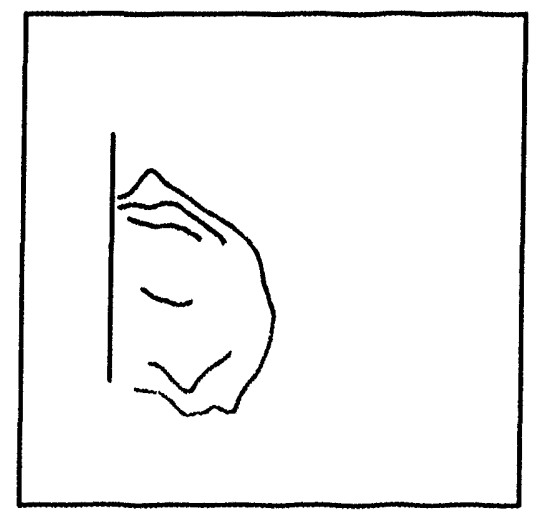

(d)

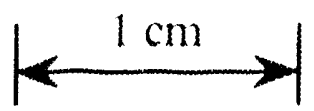

Fig. 4.14 Time scan in $52 \mathrm{~m}$ Torr argon gas. Hologram (a) taken at $\left(0.20 \mu \mathrm{s},+0.008 \mathrm{~nm}, \approx 3.9 \mathrm{~J} / \mathrm{cm}^{2}\right)$ and hologram (b) taken at $(0.50 \mu \mathrm{s}$, $+0.008 \mathrm{~nm}, \approx 3.7 \mathrm{~J} / \mathrm{cm}^{2}$ ). (c) is an equicontour plot of (a), lines correspond to increasing fringe shifts, from outer to inner line: $(0.5 \mathrm{FS}$ $\left.=9.7 \times 10^{13} \mathrm{~cm}^{-2}\right),\left(1.0 \mathrm{FS}=1.9 \times 10^{14} \mathrm{~cm}^{-2}\right)$, and $\left(1.5 \mathrm{FS}=2.9 \times 10^{14}\right.$ $\mathrm{cm}^{-2}$ ); (d) is an equicontour plot of (b), lines correspond to increasing fringe shifts, from outer to inner line: $\left(0.5 \mathrm{FS}=9.7 \times 10^{13} \mathrm{~cm}^{-2}\right),(1.0 \mathrm{FS}$ $=1.9 \times 10^{14} \mathrm{~cm}^{-2}$ ), and $\left(1.5 \mathrm{FS}=2.9 \times 10^{14} \mathrm{~cm}^{-2}\right.$ ). (From laser spots similar to \#3 - \#6 of Fig. 3.2(b).) 


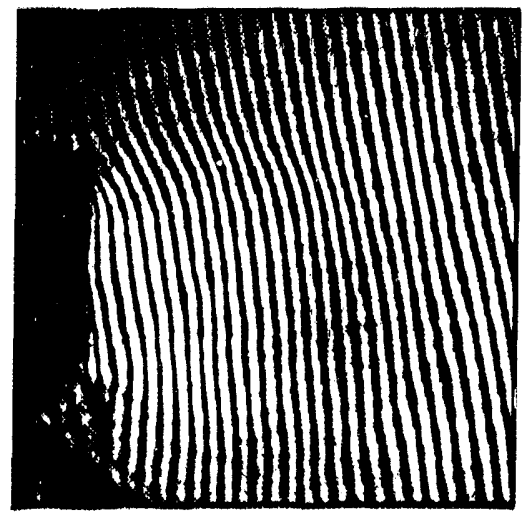

(e)

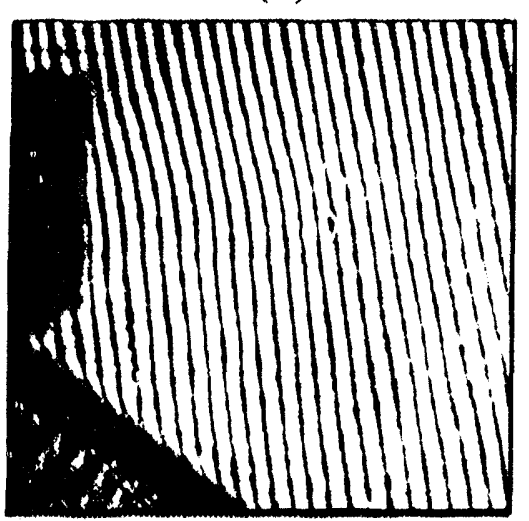

(f)

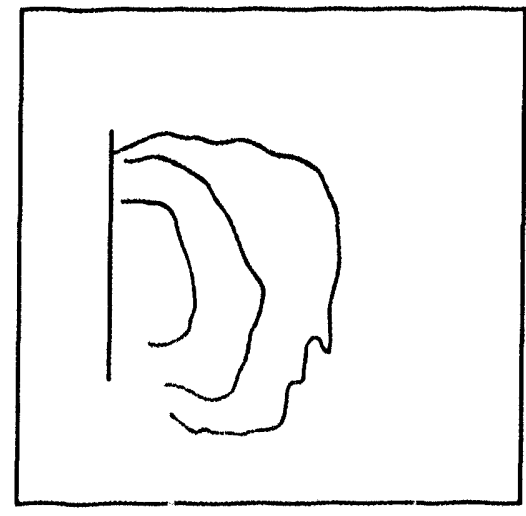

(g)

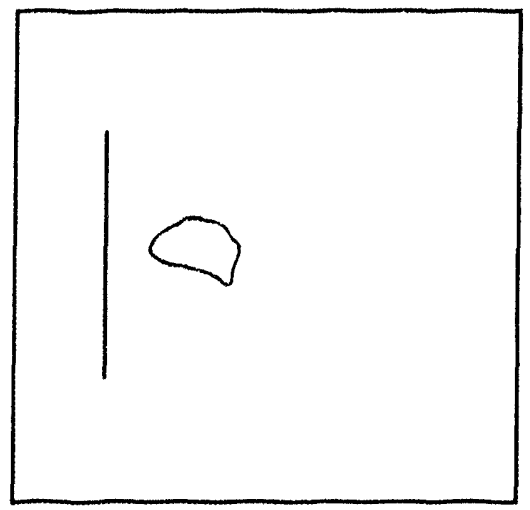

(h)

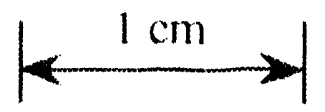

Fig. 4.14 Time scan in $52 \mathrm{~m}$ Torr, continued. Hologram (e) taken at $\left(0.80 \mu \mathrm{s},+0.008 \mathrm{~nm}, \approx 3.6 \mathrm{~J} / \mathrm{cm}^{2}\right)$ and hologram (f) taken at $(1.20 \mu \mathrm{s}$, $\left.+0.008 \mathrm{~nm}, \approx 3.5 \mathrm{~J} / \mathrm{cm}^{2}\right)$. (g) is an equicontour plot of (e), lines corre spond to increasing fringe shifts, from outer to inner line:

$\left(0.5 \mathrm{FS}=9.7 \times 10^{13} \mathrm{~cm}^{-2}\right),\left(1.0 \mathrm{FS}=1.9 \times 10^{14} \mathrm{~cm}^{-2}\right)$, and $\left(1.5 \mathrm{FS}=2.9 \times 10^{14}\right.$ $\left.\mathrm{cm}^{-2}\right)$; (h) is an equicontour plot of (f), line corresponds to (0.5 FS $=9.7 \times 10^{13} \mathrm{~cm}^{-2}$ ). (From laser spots similar to \#3-\#6 of Fig. 3.2(b).) 


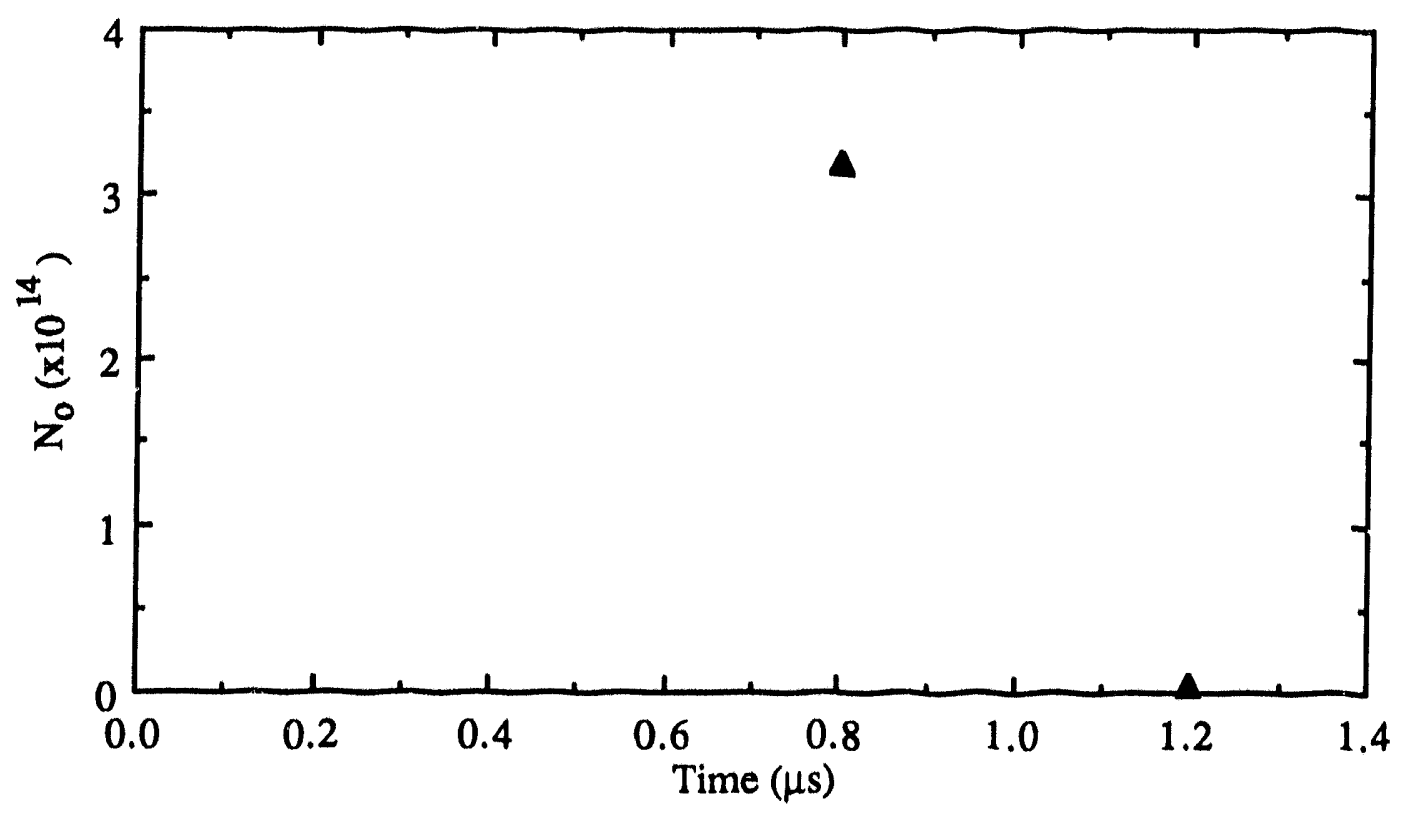

Fig. 4.15(a) Total number of aluminum neutral atoms, $\mathrm{N}_{\mathrm{o}}$, versus time in 52 mTorr argon gas.

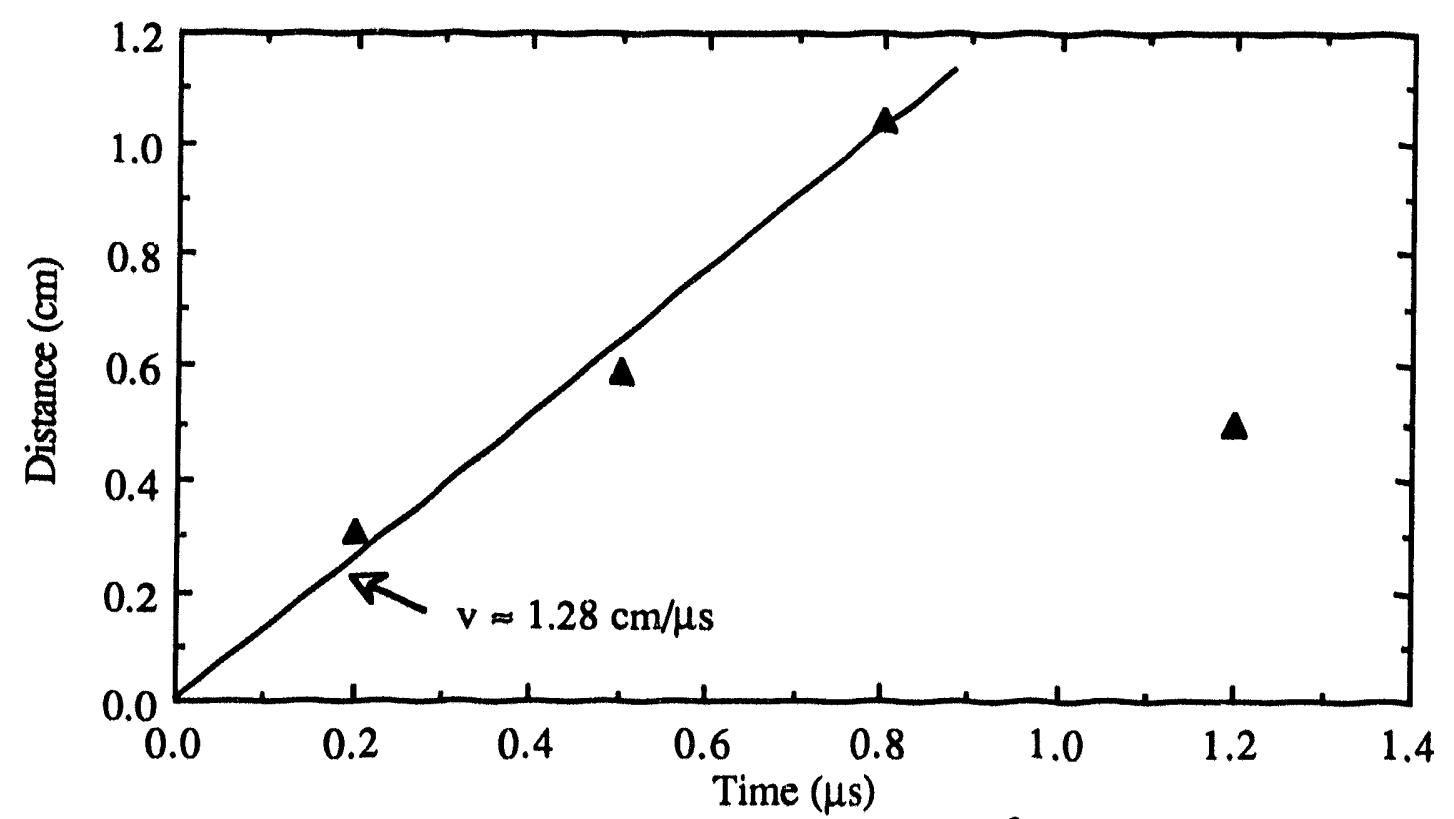

Fig 4.15(b) Expansion of the $1 \times 10^{14} \mathrm{~cm}^{-2}$ equicontour line in $52 \mathrm{~m}$ Torr argon gas. 


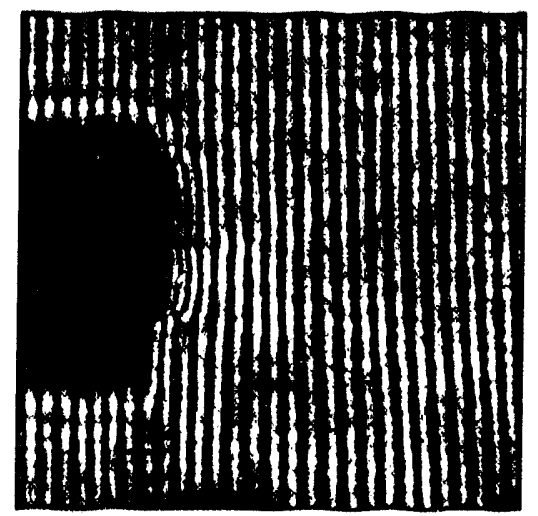

(a)

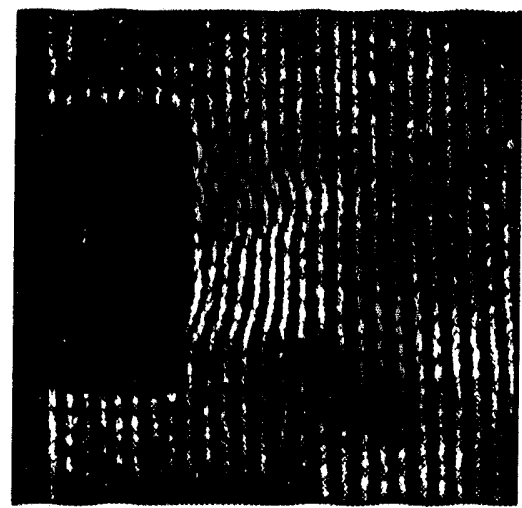

(b)

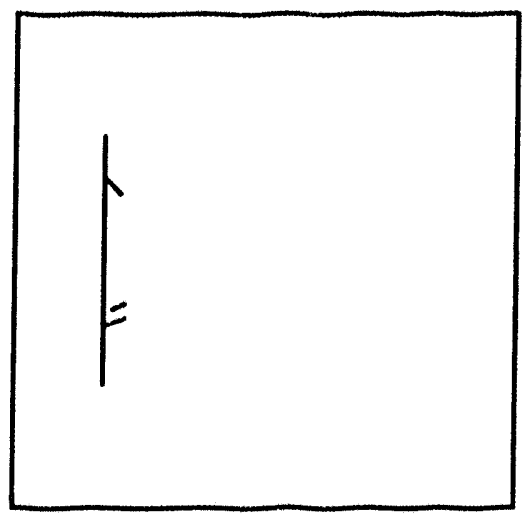

(c)

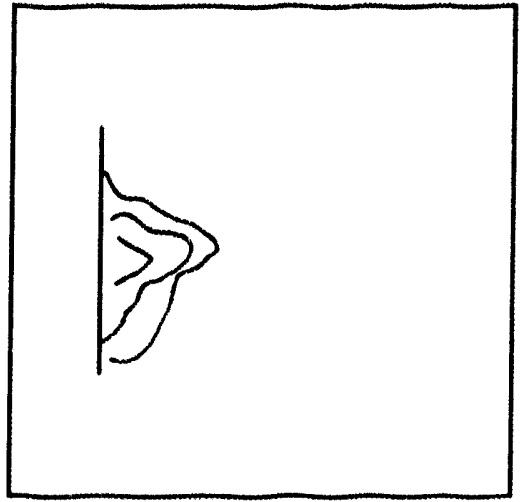

(d)

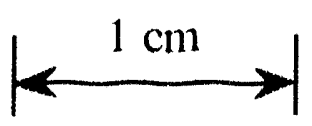

Fig. 4.16 Time scan in 210 m Torr argon gas. Hologram (a) taken at $\left(0.23 \mu \mathrm{s},-0.015 \mathrm{~nm}, \approx 4.1 \mathrm{~J} / \mathrm{cm}^{2}\right)$ and hologram (b) taken at $(0.40 \mu \mathrm{s}$, $-0.015 \mathrm{~nm}, \approx 4.7 \mathrm{~J} / \mathrm{cm}^{2}$ ). (c) is an equicontour plot of (a), lines correspond to increasing fringe shifts, from outer to inner line: $(0.5 \mathrm{FS}$ $\left.=1.9 \times 10^{14} \mathrm{~cm}^{-2}\right)$ and $\left(1.0 \mathrm{FS}=3.7 \times 10^{14} \mathrm{~cm}^{-2}\right)$; (d) is an equicontour plot of (b), lines correspond to increasing fringe shifts, from outer to inner line: $\left(0.5 \mathrm{FS}=1.9 \times 10^{14} \mathrm{~cm}^{-2}\right),\left(1.0 \mathrm{FS}=3.7 \times 10^{14} \mathrm{~cm}^{-2}\right)$, and $\left(1.5 \mathrm{FS}=5.6 \times 10^{14} \mathrm{~cm}^{-2}\right)$. (From laser spots similar to \#3 - \#6 in Fig. 3.2(b).) 


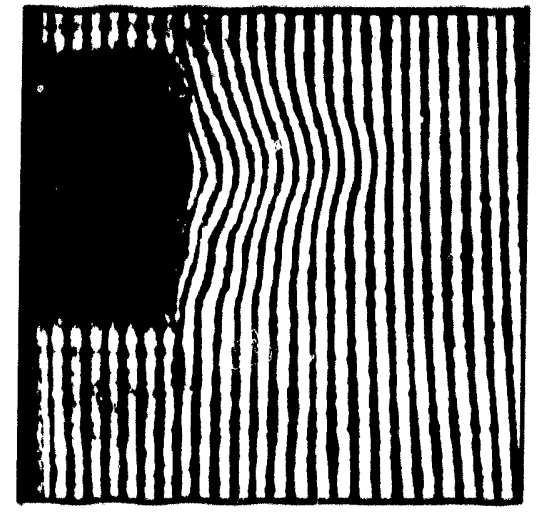

(e)

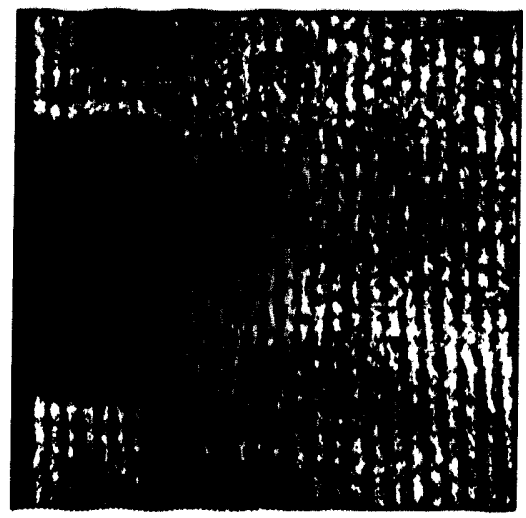

(f)

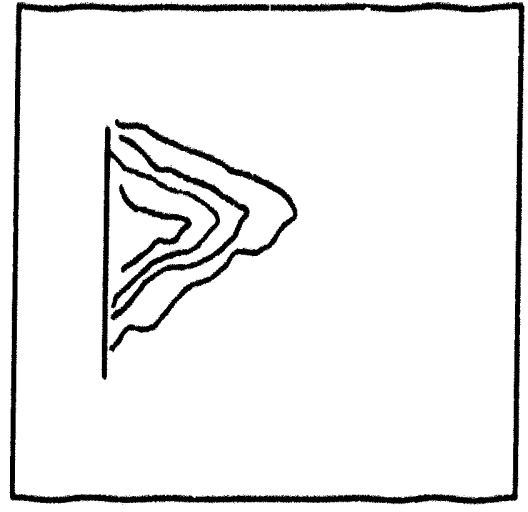

(g)

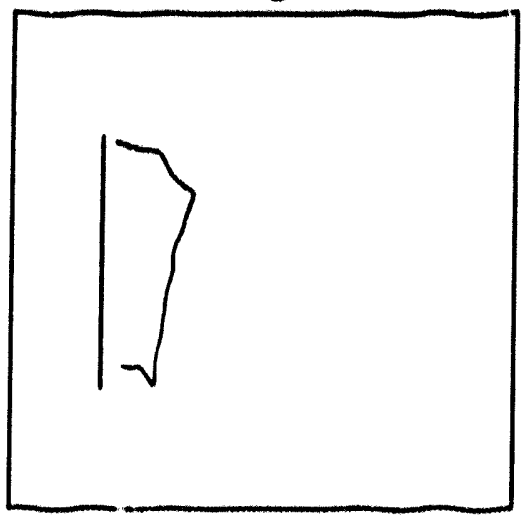

(h)

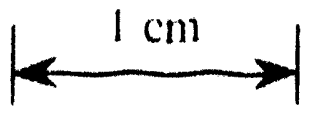

Fig. 4.16 Time scan in $210 \mathrm{mTorr}$, continued. Hologram (e) taken at $\left(0.51 \mu \mathrm{s},-0.015 \mathrm{~nm}, \approx 4.8 \mathrm{~J} / \mathrm{cm}^{2}\right)$ and hologram (f) takell at $(0.74 \mu \mathrm{s}$, $\left.-0.015 \mathrm{~nm}, \approx 4.8 \mathrm{~J} / \mathrm{cm}^{2}\right)$. (g) is an equicontour plot of (e), lines correspond to increasing fringe shifts, from outer tc inner line: $\left(0.5 \mathrm{FS}=1.9 \times 10^{14} \mathrm{~cm}^{-2}\right),\left(1.0 \mathrm{FS}=3.7 \times 10^{14} \mathrm{~cm}^{-2}\right),\left(1.5 \mathrm{FS}=5.6 \times 10^{14}\right.$ $\left.\mathrm{cm}^{-2}\right)$, and $\left(2.0 \mathrm{FS}=7.4 \times 10^{14} \mathrm{~cm}^{-2}\right) ;(\mathrm{h})$ is an equicontour plot of $(\mathrm{f})$, line corresponds to $\left(0.5 \mathrm{FS}=1.9 \times 10^{14} \mathrm{~cm}^{-2}\right)$. (From laser spots similar to \#3 - \#6 of Fig, 3.2(b).) 


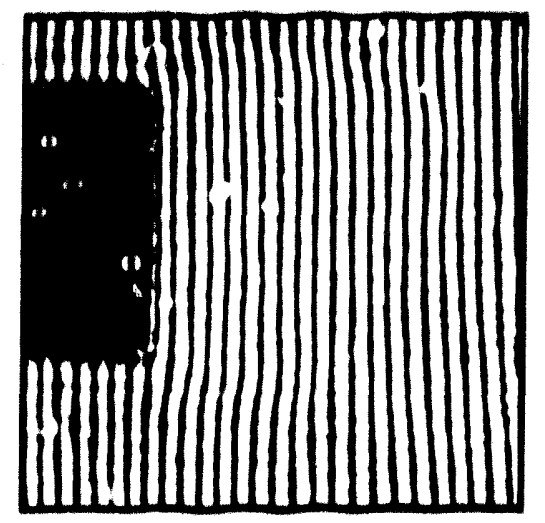

(i)

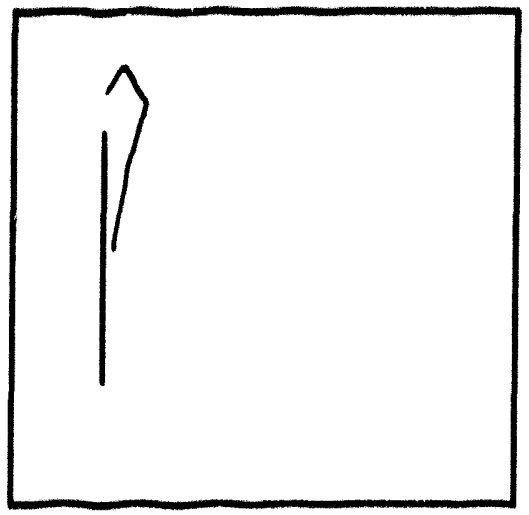

(j)

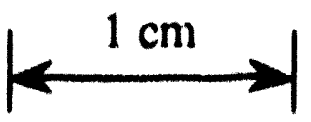

Fig. 4.16 Time scan in 210 mTorr, continued. Hologram (i) taken at $\left(1.24 \mu \mathrm{s},-0.015 \mathrm{~nm}, \approx 4.9 \mathrm{~J} / \mathrm{cm}^{2}\right)$. (j) is an equicontour plot of (i), line corresponds to $\left(0.5 \mathrm{FS}=1.9 \times 10^{14} \mathrm{~cm}^{-2}\right)$. (From laser spots similar to \#3 - \#6 of Fig. 3.2(b).) 


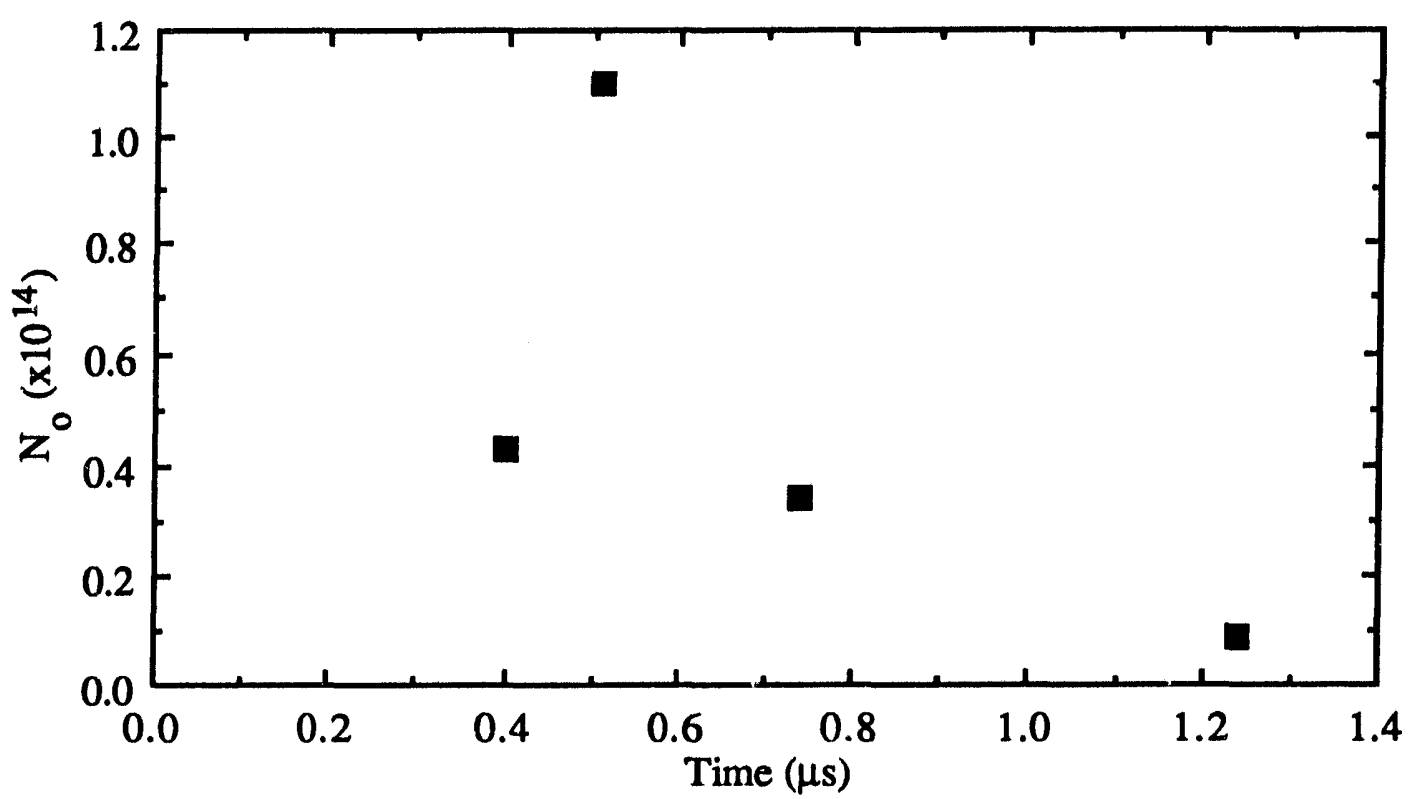

Fig. 4.17(a) Total number of aluminum neutral atoms, $\mathrm{N}_{\mathrm{o}}$, versus time in $210 \mathrm{mTorr}$ argon gas.

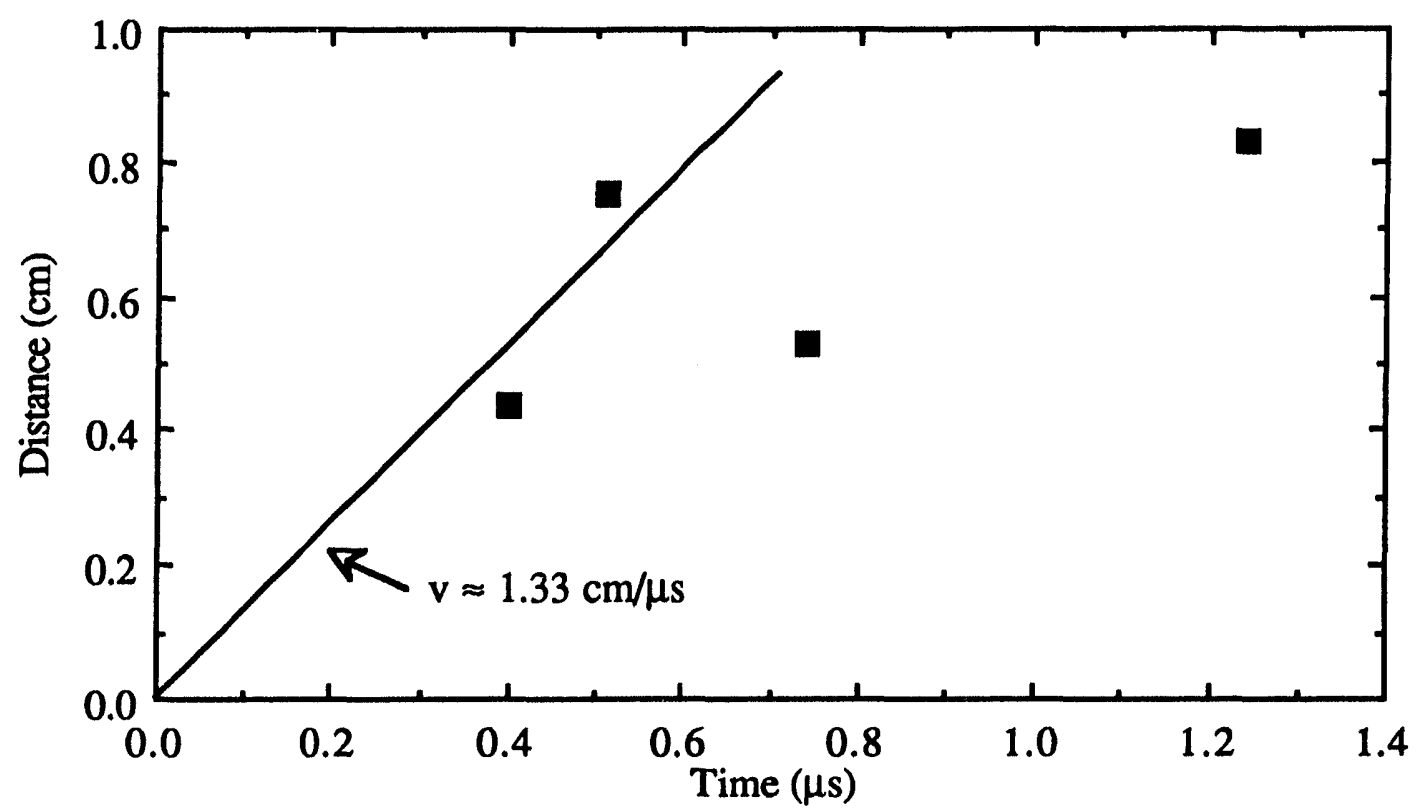

Fig. 4.17(b) Expansion of the $1 \times 10^{14} \mathrm{~cm}^{-2}$ equicontour line in $210 \mathrm{mTorr}$ argon gas. 


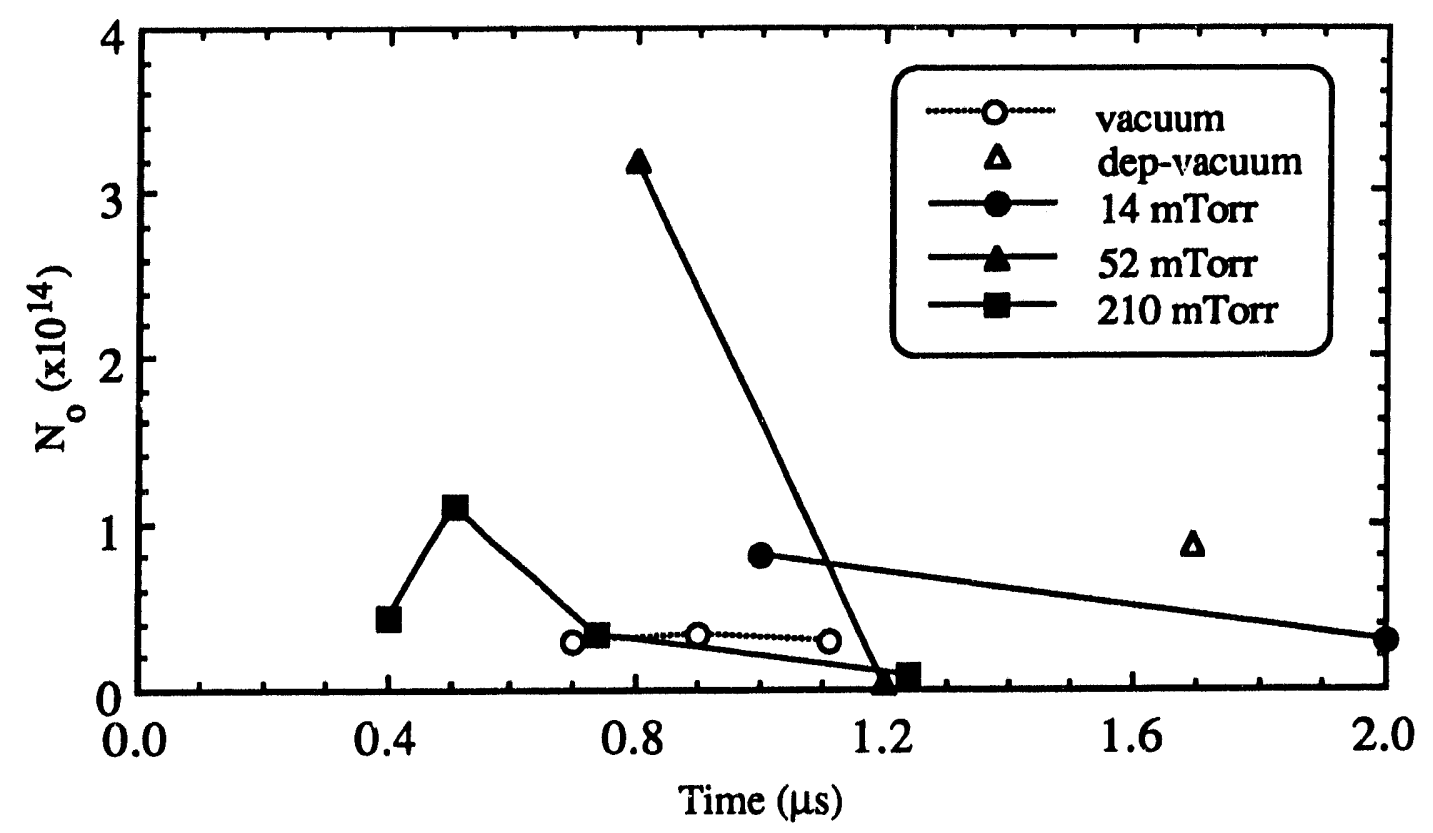

Fig. 4.18(a) Total number of neutral aluminum atoms, $N_{0}$, versus time in vacuum and lower pressure argon gas backgrounds.

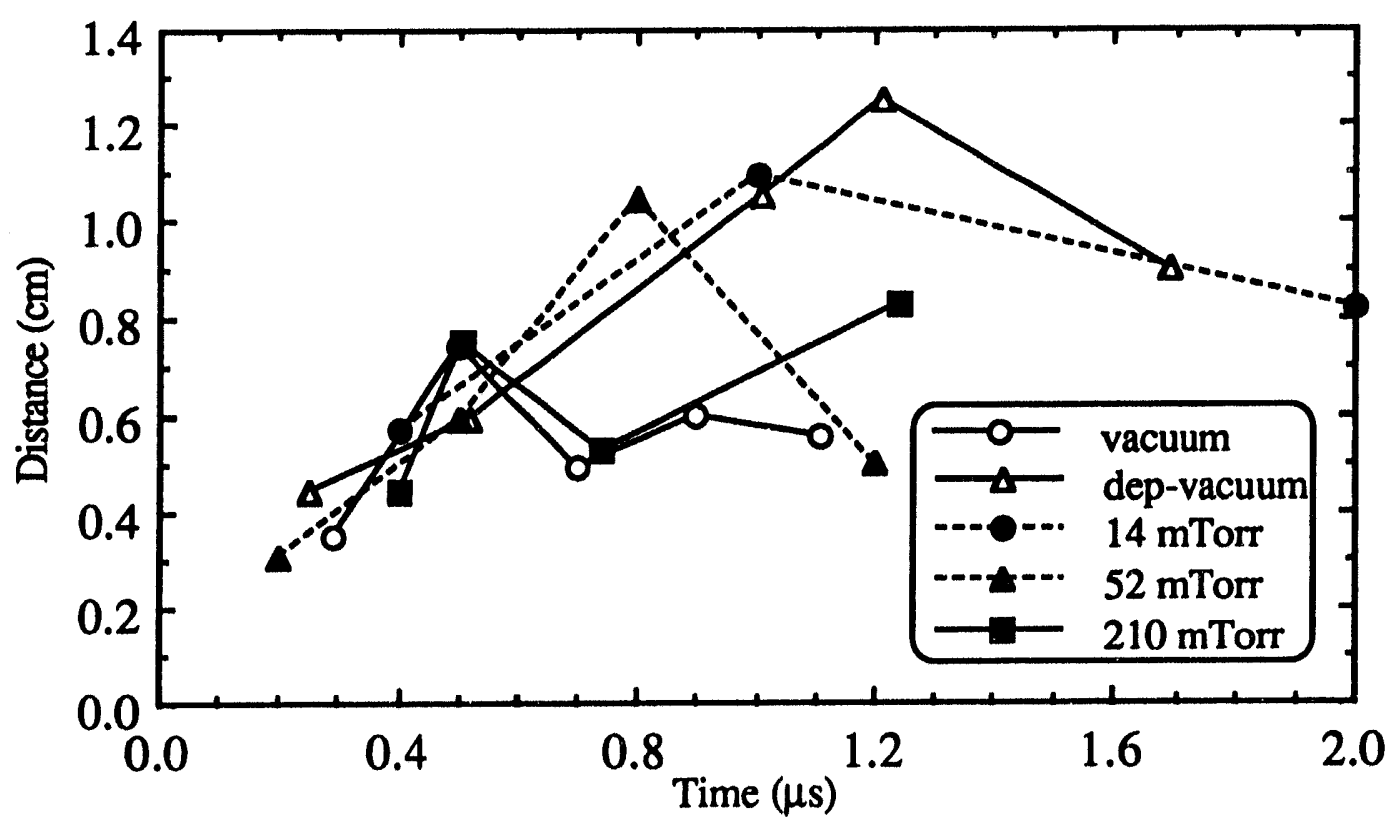

Fig. 4.18(b) Expansion of the $1 \times 10^{14} \mathrm{~cm}^{-2}$ equicontour line in vacuum and lower pressure argon gas backgrounds. 


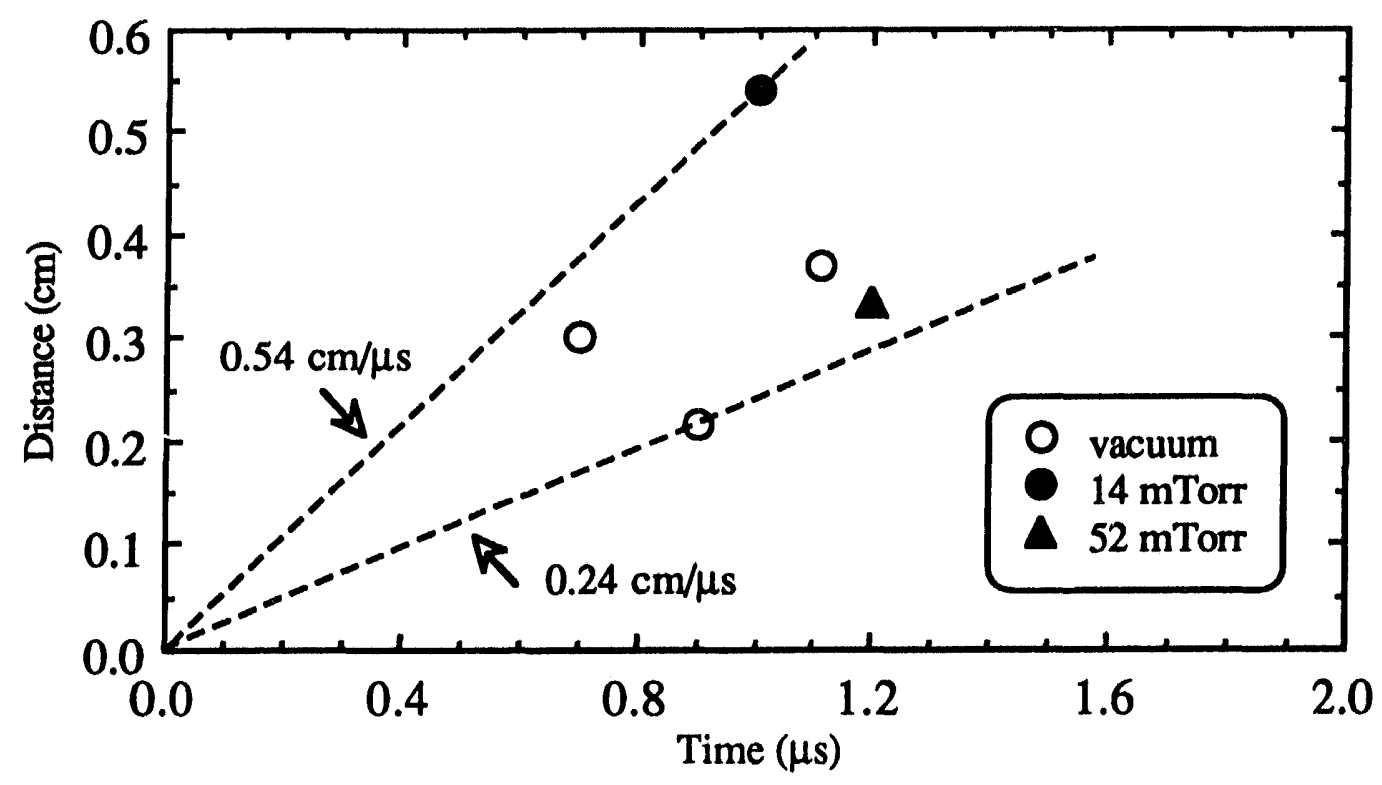

Fig. 4.19 Expansion of the ablation plume center in vacuum and lower pressure argon gas backgrounds.

$$
\mathrm{k}_{\mathrm{B}} \mathrm{T}=\frac{(\gamma-1)^{2}}{2 \gamma} * \frac{\mathrm{m} \mathrm{u}_{\max }^{2}}{2}
$$

where $\gamma=1.667$ for an ideal, monoatomic gas and $u_{\max }$ is the maximum expansion velocity measured for the laser ablation plume after the plume has become non-collisional. The velocities $1.05 \mathrm{~cm} / \mu \mathrm{s}$ and $1.41 \mathrm{~cm} / \mu \mathrm{s}$ then relate to initial plume temperatures $2.1 \mathrm{eV}$ and $3.7 \mathrm{eV}$, respectively. This falls within the temperature range measured by Dreyfus using LIF on copper ablation for similar fluences: $\approx 0.26 \mathrm{eV}$ (Dre91) to $\approx 4 \mathrm{eV}$ (Dre86).

As another indicator of the rate of plume expansion, Figure 4.19 shows the expansion of the peak aluminum neutral line density (after it has separated from the target surface) as a function of time. The points on the graph all fall within the average velocity range of $0.24 \mathrm{~cm} / \mu \mathrm{s}$ - 
$0.54 \mathrm{~cm} / \mu \mathrm{s}$, which is only somewhat slower than the expansion of the $1 \times 10^{14} \mathrm{~cm}^{-2}$ equicontour line.

It is important to clarify again that the perpendicular expansion velocities $\left(\mathbf{v}_{\mathbf{z}}\right)$ are larger than the parallel expansion velocities $\left(\mathbf{v}_{\mathbf{x}}, \mathbf{v}_{\mathbf{y}}\right)$, as seen in the holograms, and that even the two parallel expansion velocities $\left(v_{x}, v_{y}\right)$ are different from each other, as seen by the shape of the deposited thin film. Since the dye laser passes parallel to the target surface, it is the ensemble, parallel expansion velocity distribution that is desired for the fringe shift interpretation calculations.

Dreyfus measured the perpendicular expansion temperature (corresponding to $\mathrm{v}_{\mathrm{z}}$ ) to be in the range $0.20 \mathrm{eV}-4.0 \mathrm{eV}$ (Dre86, Dre91). The perpendicular expansion temperature range measured here, $2.1 \mathrm{eV}-3.7 \mathrm{eV}$, is consistent with Dreyfus' measurements, and the parallel expansion temperature for the ablation plumes in this work is presumed to be in the range $0.1 \mathrm{eV}-1.0 \mathrm{eV}$. Since the plume temperature was not measured directly for the ablation plumes in this work, Dreyfus' lower value, $0.3 \mathrm{eV}$, was chosen as the value to use in the fringe shift interpretation calculations. Finally, it was shown in Chapter 2 that the resonant hologram interpretation calculation is insensitive to temperature for temperatures within the range $0.1 \mathrm{eV}-1.0 \mathrm{eV}$.

\section{Theoretically Modeling Plume Expansion In Vacuum And Low Pressure, Inert, Background Gas}

Laser ablation plume expansion in vacuum (or collisionless transport of the plume through the background gas) has been modeled 
theoretically by Utterback, et al. (Utt76), Talents (Tal83), and Kools, et al. (Koo92) to have the form

$$
n(x, y, z)=\frac{N_{o}\left(\frac{m}{2 \pi k_{B}}\right)^{3 / 2}}{\left(T_{x} T_{y} T_{z}\right)^{1 / 2}\left(t^{3}\right)} \operatorname{Exp}\left\{-\frac{m}{2 k_{B} t^{2}}\left[\frac{x^{2}}{T_{x}}+\frac{y^{2}}{T_{y}}+\frac{(z-u t)^{2}}{T_{z}}\right]\right\}
$$

where $\mathrm{n}$ is the particle density, $t$ is time, $T_{X}, T_{y}$, and $T_{Z}$ are the expansion temperatures in the center of mass frame of reference, and $u$ is the center of mass velocity. Integration over the $\mathrm{x}$-direction (the direction of travel for the dye laser beam) gives

$$
\overline{n l}(y, z)=\frac{N_{o}\left(\frac{m}{2 \pi k_{B}}\right)}{\left(T_{y} T_{z}\right)^{1 / 2}\left(t^{2}\right)} \operatorname{Exp}\left\{-\frac{m}{2 k_{B} t^{2}}\left[\frac{y^{2}}{T_{y}}+\frac{(z-u t)^{2}}{T_{z}}\right]\right\}
$$

where $\overline{\mathrm{n}}$ is the line-integrated-particle-density. The plume expansion in Figures $4.6,4.8,4.12,4.14$ and 4.16 can be fit to this equation by assuming $y=0$ along the line of symmetry of the plume, rearranging the terms and taking the natural logarithm to obtain the form

$$
\ln \left[\overline{\mathrm{nl}} \mathrm{t}^{2}\right]=A\left(\frac{z}{\mathrm{t}}\right)^{2}+B\left(\frac{z}{t}\right)+C
$$

where $\mathrm{z}$ is the maximum expansion distance of the equicontour with a line density $\bar{n}$, 


$$
A=\frac{-m}{2 k_{B} T_{z}} \quad B=-2 A u \quad \text { and } \quad C=\ln \left[\frac{m N_{o}}{2 \pi k_{B} \sqrt{T_{y} T_{z}}}\right]+A u^{2}
$$

When $\ln \left[\bar{n}{ }^{*} t^{2}\right]$ vs $(z / t)$ is graphed for a given hologram, the points are binomially fit for the parameters A, B and C, from which the parameters $T_{z}, u$, and $N d \sqrt{T_{y}}$ can be calculated. While the graphs of $\ln \left[\bar{n}^{*} t^{2}\right]$ vs $(\mathrm{z} / \mathrm{t})$ have been relegated to Appendix 6, the latter three parameters are graphed versus time in Figures 4.20, 4.21 and 4.22.

First of all, it is necessary to have at least three equicontour lines for each hologram in order to fit a binomial curve to its graph of $\ln \left[\bar{n} 1 * t^{2}\right]$ vs $(z / t)$. Three of the five time scans only had one hologram that met this requirement and so these time scans only have one point each in Figures 4.20, 4.21 and 4.22 .

Secondly, it is important to remember that the parameters presented in these figures are not directly measured, they are only inferred from the analysis described above and could be used to recreate a theoretical surface plot with a similar appearance to the presented hologram tracings. Third, the expansion model assumes a point source, while the source of ablated particles in this experiment is about 0.05 $0.10 \mathrm{~cm}^{2}$.

Fourth, notice that the inferred center of mass velocity decreases quickly in time and is even negative for two holograms, while the inferred plume expansion temperature, $T_{z}$, increases rapidly in time. These results conflict with the previously made assumptions that the plume is collisionless with the background gas (and thus the center of 


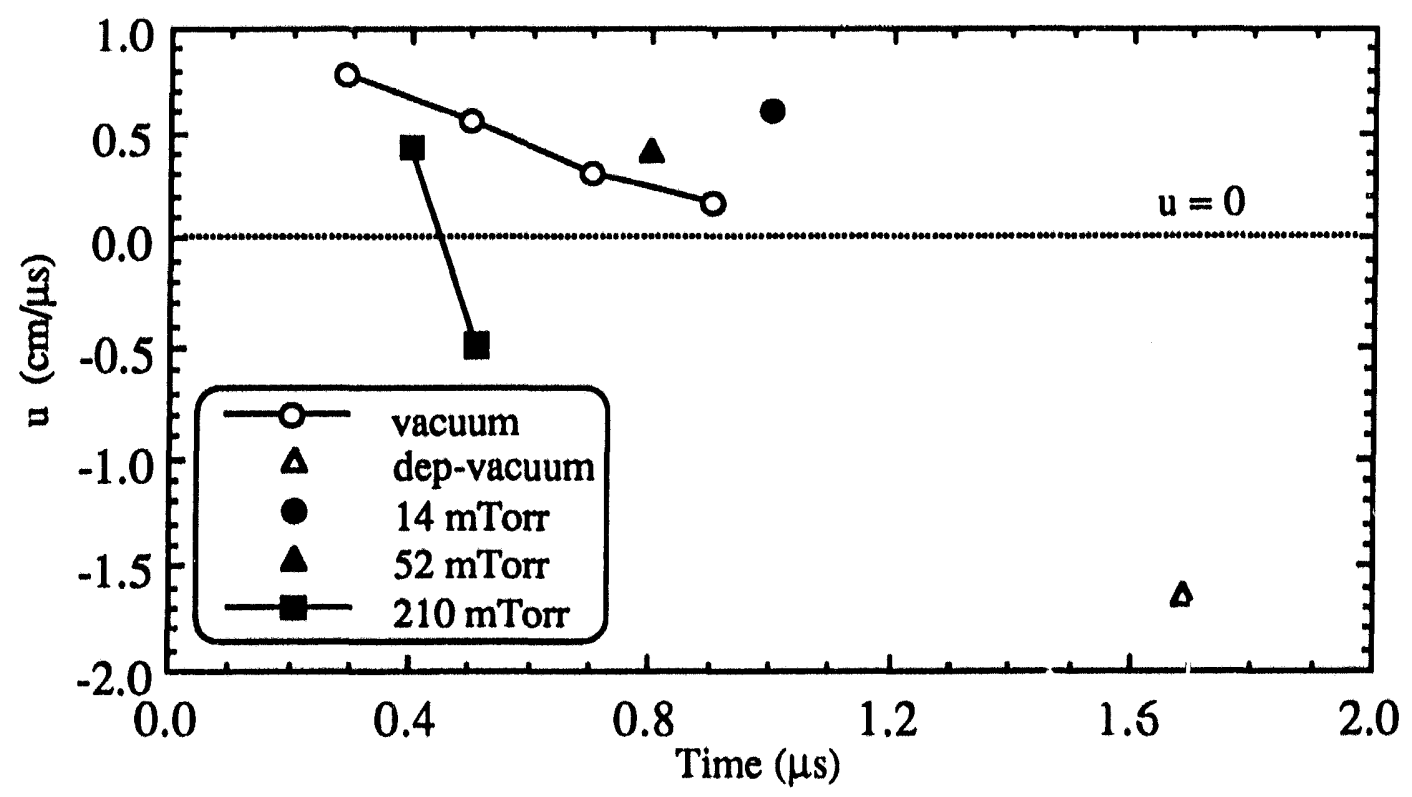

Fig. 4.20 Inferred center of mass velocity, $u$, versus time for vacuum and lower pressure argon gas backgrounds.

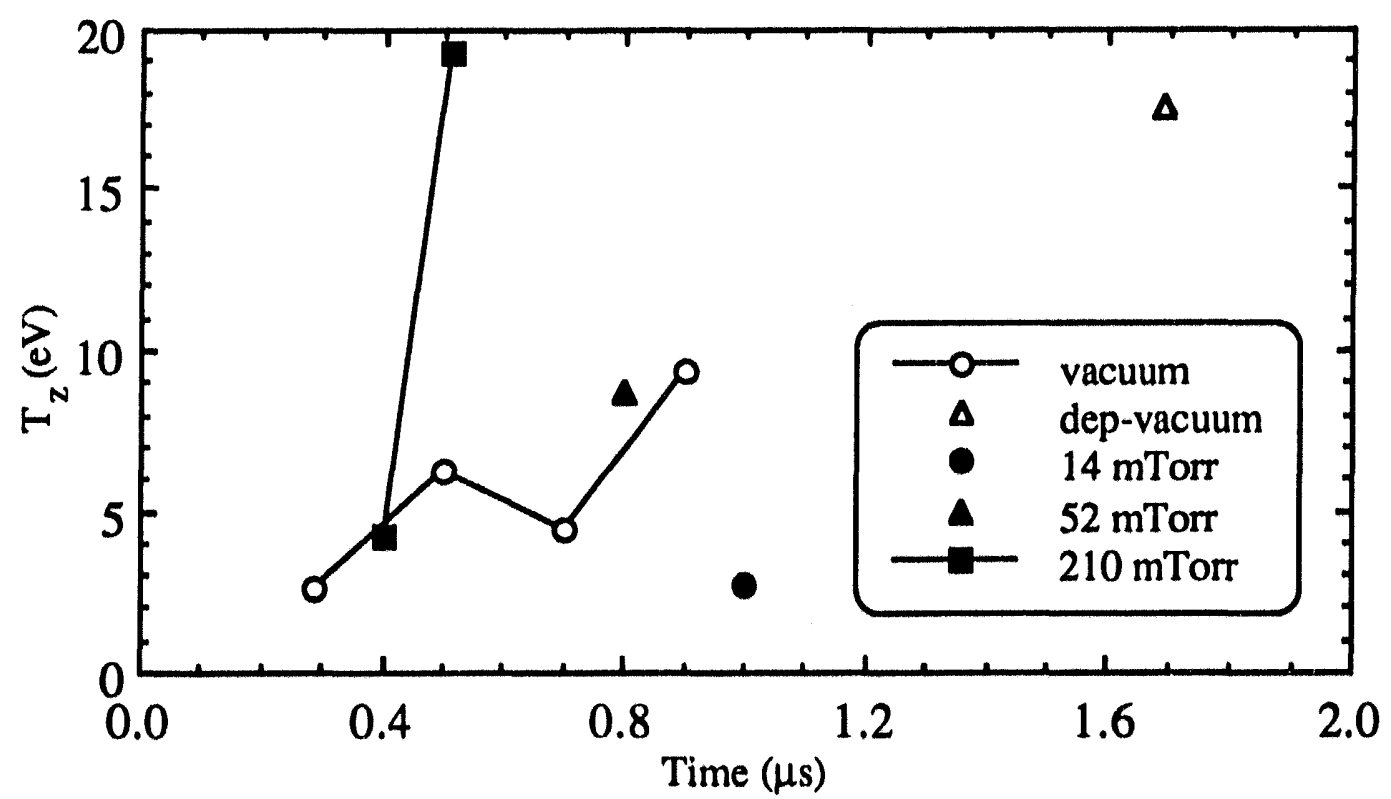

Fig. 4.21 Inferred, perpendicular plume expansion temperature, $T_{z}$, versus time in vacuum and lower pressure argon gas backgrounds. 


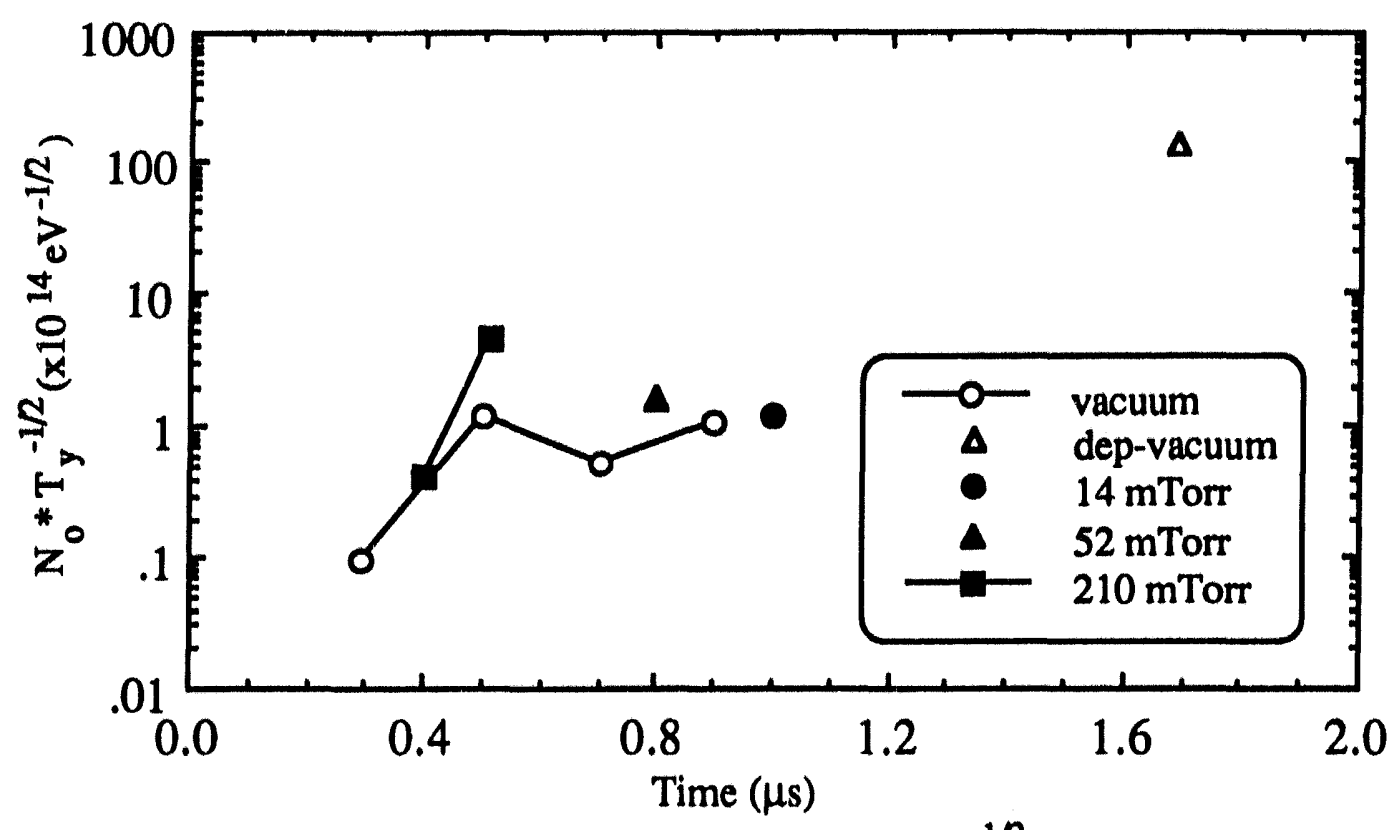

Fig. 4.22 The inferred parameter, $\mathrm{N}_{\mathrm{o}} \times \mathrm{T}_{y}{ }^{-1 / 2}$, versus time in vacuum and lower pressure argon gas backgrounds.

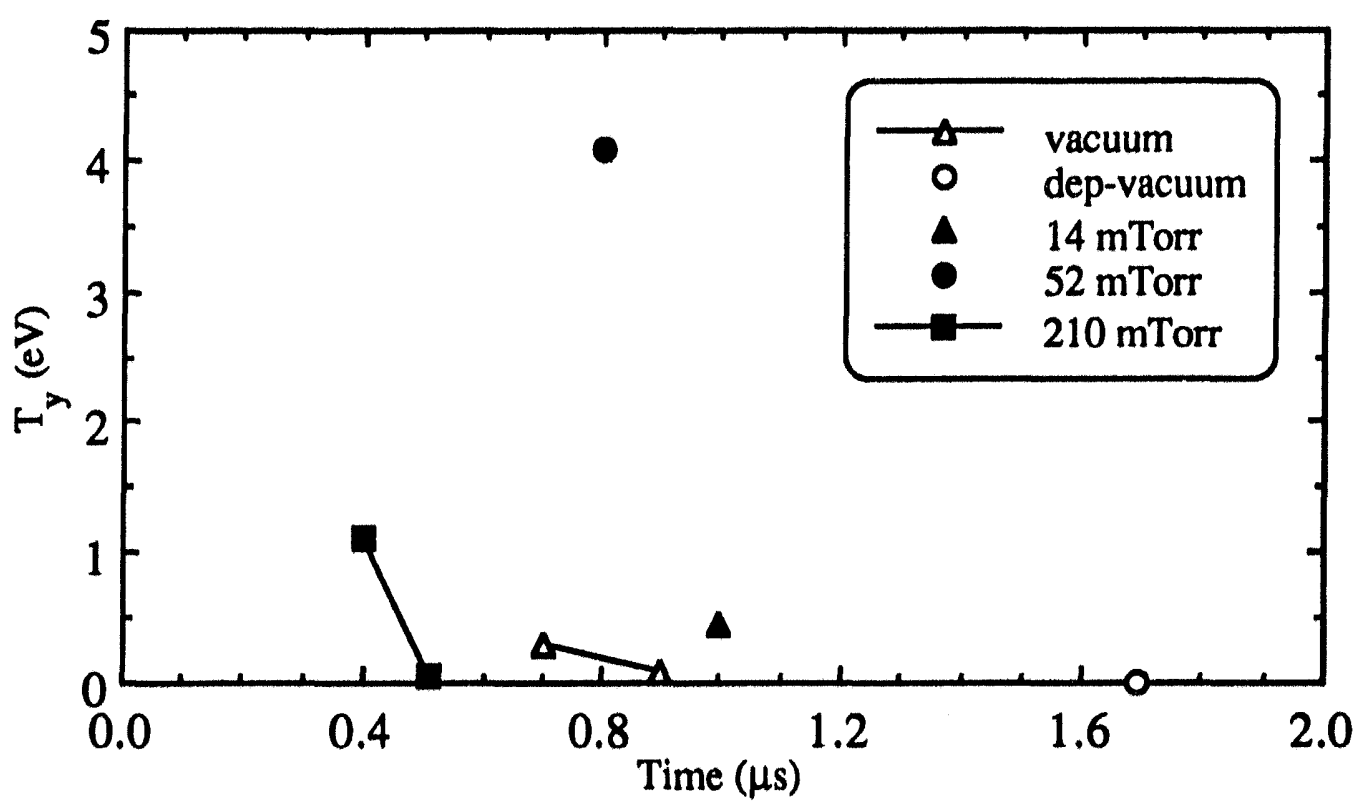

Fig. 4.23 Inferred plume expansion temperature, $\mathrm{T}_{\mathrm{y}}$, versus time in vacuum and lower pressure argon gas backgrounds. (Combination of Fig. 4.18(a) and Fig. 4.22.) 
mass velocity does not decrease rapidly) and that the plume temperature (velocity distribution) is not changing with time because, again, the plume is collisionless during the time of investigation. Instead, these graphs are an indication that the plume expansion model does not fit the holographic data well. In practice, there probably exist two groups of particles (components) in the ablation plume and the above model only describes the motion of one of those components.

One group of particles (ions and charge exchange neutrals accelerated by the ambipolar potential) energetically moves away from the target and undergoes self-similar expansion according to Equation 4.5 while the other group of particles stays very close (within a few $\mathrm{mm}$ ) to the target surface. Hereafter, these will be called the expanding plume and the residual plume for simplicity.

The expanding plume and the residual plume have been observed and discussed for laser ablation in vacuum by Geohegan using emission spectroscopy (Geo92, Geo93) and by Bakos, et al. using TOFPRL (Bak87). The phenomena is also visible in the PLIF data discussed by Cappelli, et al. (Cap90). However, this should not be confused with discussions distinguishing between species within the expanding plume such as Saenger, who discussed the differential expansion of the four different kinds of atoms in the ablation of $\mathrm{YBaCuO}$ (Sae91), or Kools, et al., who observed a $\mathrm{CuCl}$ expanding plume and a slower, $\mathrm{Cu}_{3} \mathrm{Cl}_{3}$ expanding plume following the ablation of copper in the presence of chlorine gas (Koo92).

Therefore, the holograms in Figures 4.6, 4.8, 4.12, 4.14 and 4.16 represent the RHI-detectable portion of the sum of the expanding plume and the residual plume. In most cases, however, the expanding plume is 
not detectable after separation from the residual plume. For example, Fig. 4.12(d) clearly shows a dominant, expanding plume at $1.00 \mu \mathrm{s}$, while at $2.00 \mu \mathrm{s}$ in Fig. 4.12(f), the expanding plume is no longer distinct and the most dense region is next to the target. The single exception to this in the data presented thus far is Fig. 4.14(h), where the residual plume is no longer detectable and a detectable expanding plume has separated from the target at $1.20 \mu \mathrm{s}$.

For completeness, the parameter $\mathrm{N}_{\mathrm{o}} / \sqrt{\mathrm{T}_{\mathrm{y}}}$ is graphed versus time in Fig. 4.22 and $\mathrm{T}_{\mathrm{y}}$ is then calculated using the values of $\mathrm{N}_{0}$ presented in Fig. 4.18(a) and graphed versus time in Fig. 4.23. As presented above, caution should be used when interpreting these numbers, especially because $\mathrm{N}_{\mathrm{d}} / \sqrt{\mathrm{T}_{\mathrm{y}}}$ is proportional to $\operatorname{Exp}(\mathrm{C})$ as given in Eqn. 4.7.

\section{Laser Ablation In Higher Pressure, Inert, Background Gas}

Figure 4.24 shows a RHI time scan in 1 Torr argon gas. The first thing to notice is that with a high enough background pressure, a shock wave was observed at $1.02 \mu \mathrm{s}$ and $4.00 \mu \mathrm{s}$. Previously, DL.RAP studies have only detected shock waves down to pressures of $\approx 5$ Torr (Ven92). It is presumed that a shock wave is present at the other times, but that for some reason it was not detected as clearly as in Figs. 4.24(a) and (f). Secondly, notice that the plume expansion has slowed considerably with respect to the previous time scans at lower pressures.

Third, note the mushroom shape of the ablation plume. Sappey and Gamble attribute this shape for copper ablation plumes to the exothermic 


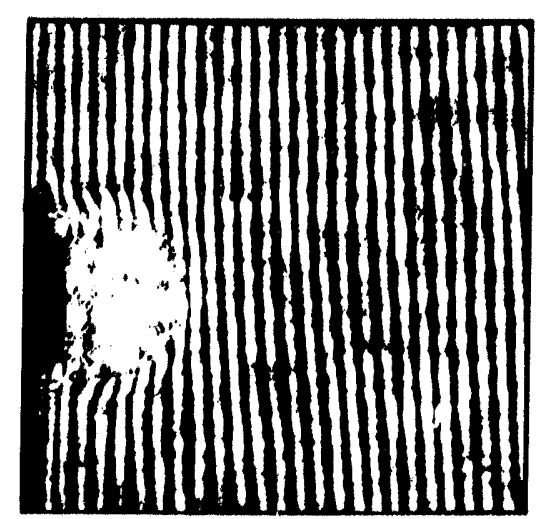

(a)

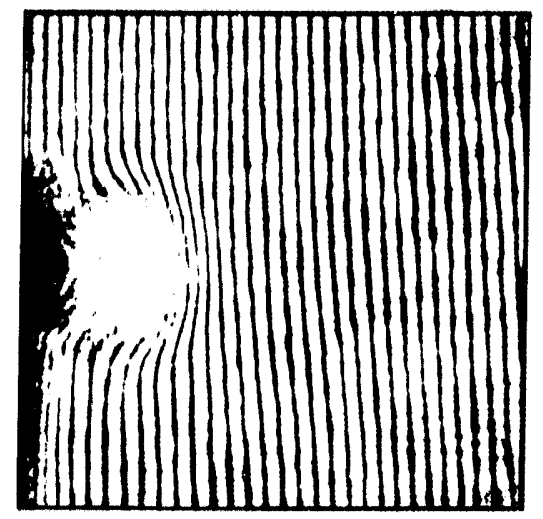

(b)

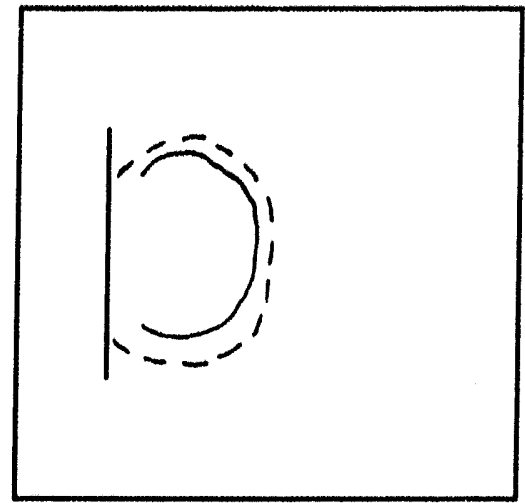

(c)

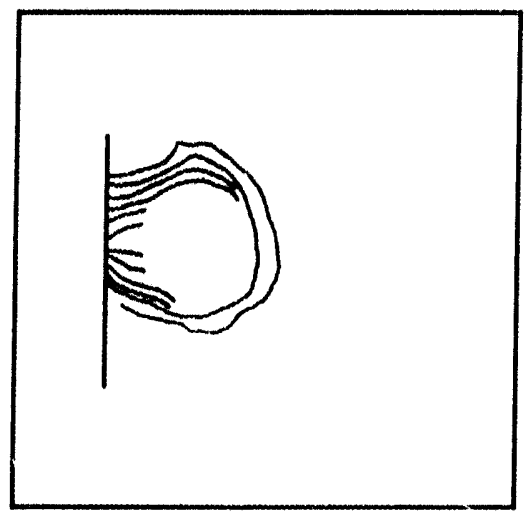

(d)

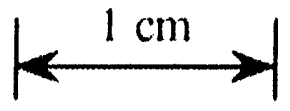

Fig. 4.24 Time scan in 1 Torr argon gas. Hologram (a) taken at $\left(1.02 \mu \mathrm{s},-0.006 \mathrm{~nm}, \approx 2.3 \mathrm{~J} / \mathrm{cm}^{2}\right)$ and hologram (b) taken at $(2.02 \mu \mathrm{s}$, $-0.006 \mathrm{~nm}, \approx 2.4 \mathrm{~J} / \mathrm{cm}^{2}$ ). (c) is an equicontour plot of (a), dashed line corresponds to a shock wave and solid line corresponds to (0.5 FS $\left.=7.0 \times 10^{13} \mathrm{~cm}^{-2}\right)$; (d) is an equicontour plot of (b), lines correspond to increasing fringe shifts, from outer to inner line: $\left(0.5 \mathrm{FS}=7.0 \times 10^{13}\right.$ $\left.\mathrm{cm}^{-2}\right),\left(1.0 \mathrm{FS}=1.4 \times 10^{14} \mathrm{~cm}^{-2}\right),\left(1.5 \mathrm{FS}=2.1 \times 10^{14} \mathrm{~cm}^{-2}\right),(2.0 \mathrm{FS}$ $\left.=2.9 \times 10^{14} \mathrm{~cm}^{-2}\right),\left(2.5 \mathrm{FS}=3.6 \times 10^{14} \mathrm{~cm}^{-2}\right)$, and $\left(3.0 \mathrm{FS}=4.4 \times 10^{14}\right.$ $\mathrm{cm}^{-2}$ ). (From laser spot \#2 of Fig. 3.2(b).) 


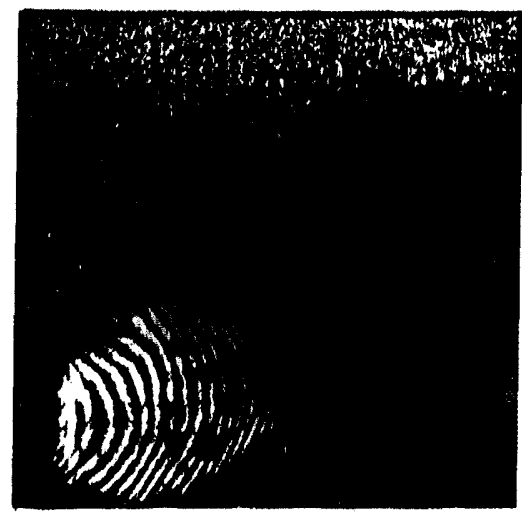

(e)

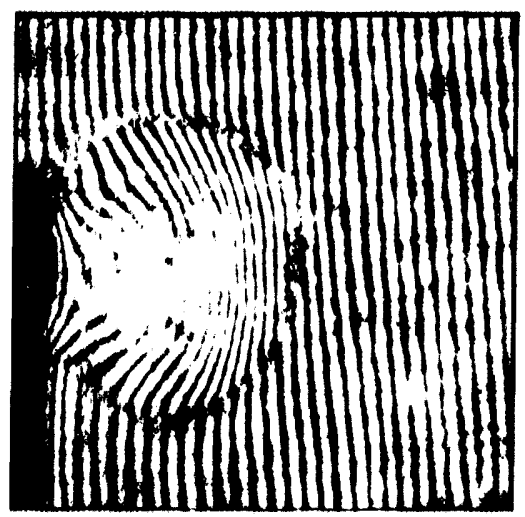

(f)

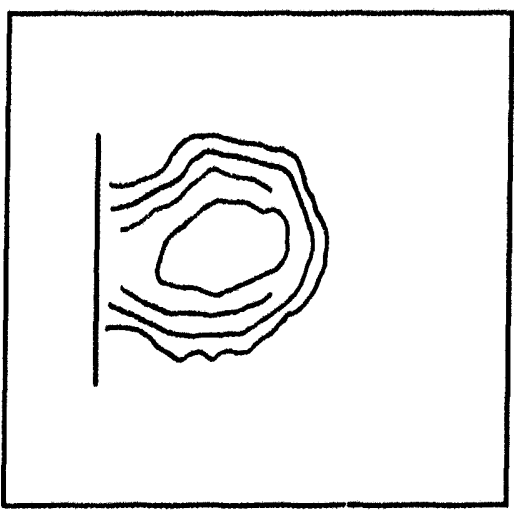

(g)

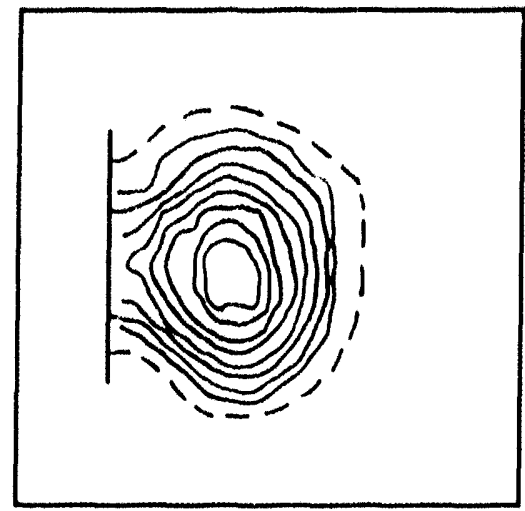

(h)

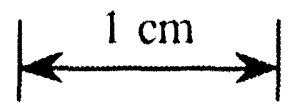

Fig. 4.24 Time scan in 1 Torr argon gas, continued. Hologram (e) taken at $\left(2.50 \mu \mathrm{s},-0.006 \mathrm{~nm}, \approx 2.1 \mathrm{~J} / \mathrm{cm}^{2}\right)$ and hologram (f) taken at $\left(4.00 \mu \mathrm{s},-0.006 \mathrm{~nm}, \approx 2.4 \mathrm{~J} / \mathrm{cm}^{2}\right)$. $(\mathrm{g})$ is an equicontour plot of (e); lines correspond to increasing fringe shifts, from outer to inner line: $\left(1.0 \mathrm{FS}=1.4 \times 10^{14} \mathrm{~cm}^{-2}\right),\left(2.0 \mathrm{FS}=2.9 \times 10^{14} \mathrm{~cm}^{-2}\right),\left(3.0 \mathrm{FS}=4.4 \times 10^{14}\right.$ $\left.\mathrm{cm}^{-2}\right)$, and $\left(4.0 \mathrm{FS}=6.0 \times 10^{14} \mathrm{~cm}^{-2}\right)$. (h) is an equicontour plot of $(\mathrm{f})$; dashed line corresponds to a shock wave and solid lines correspond to increasing fringe shifts, from outer to inner line: $\left(0.5 \mathrm{FS}=7.0 \times 10^{13}\right.$ $\left.\mathrm{cm}^{-2}\right),\left(1.0 \mathrm{FS}=1.4 \times 10^{14} \mathrm{~cm}^{-2}\right),\left(1.5 \mathrm{FS}=2.1 \times 10^{14} \mathrm{~cm}^{-2}\right),(2.0 \mathrm{FS}$ $\left.=2.9 \times 10^{14} \mathrm{~cm}^{-2}\right),\left(2.5 \mathrm{FS}=3.6 \times 10^{14} \mathrm{~cm}^{-2}\right),\left(3.0 \mathrm{FS}=4.4 \times 10^{14} \mathrm{~cm}^{-2}\right)$, $\left(3.5 \mathrm{FS}=5.2 \times 10^{14} \mathrm{~cm}^{-2}\right)$ and $\left(4.0 \mathrm{FS}=6.0 \times 10^{14} \mathrm{~cm}^{-2}\right.$ ). (From laser spot \#2 of Fig. 3.2(b).) 


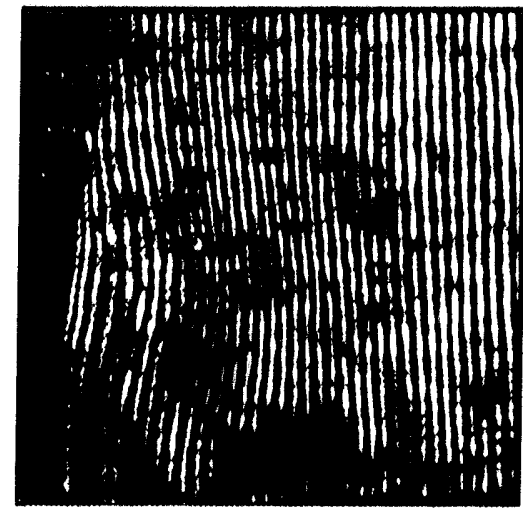

(i)

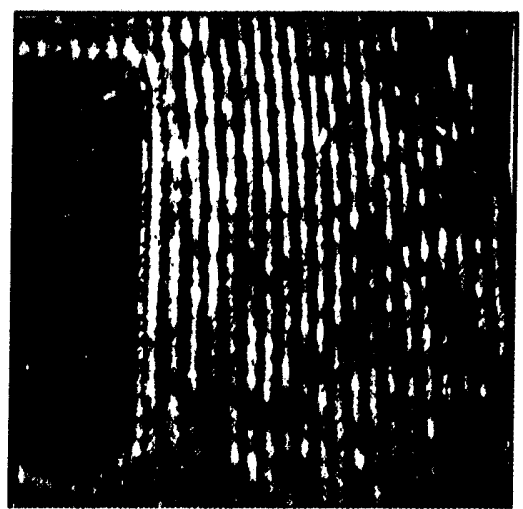

(j)

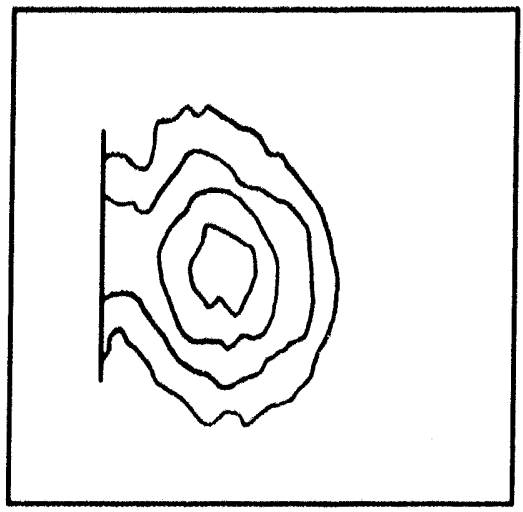

(k)

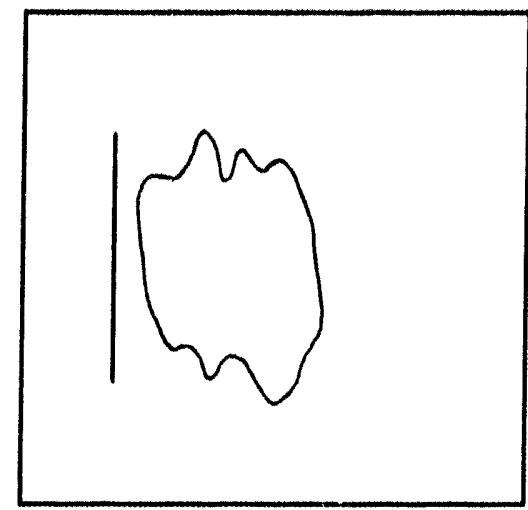

(1)

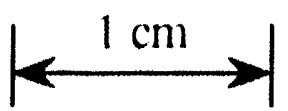

Fig. 4.24 Time scan in 1 Torr argon gas, continued. Hologram (i) laken at $\left(10.0 \mu \mathrm{s},-0.006 \mathrm{~nm}, \approx 2.4 \mathrm{~J} / \mathrm{cm}^{2}\right)$ and hologram $(\mathrm{j})$ taken at $\left(20.0 \mu \mathrm{s},-0.006 \mathrm{~nm}, \approx 2.4 \mathrm{~J} / \mathrm{cm}^{2}\right)$. (k) is an equicontour plot of (i), lines correspond to increasing fringe shifts, from outer to inner line: $\left(0.5 \mathrm{FS}=7.0 \times 10^{13} \mathrm{~cm}^{-2}\right),\left(1.0 \mathrm{FS}=1.4 \times 10^{14} \mathrm{~cm}^{-2}\right),\left(1.5 \mathrm{FS}=2.1 \times 10^{14}\right.$ $\left.\mathrm{cm}^{-2}\right)$, and $\left(2.0 \mathrm{FS}=2.9 \times 10^{14} \mathrm{~cm}^{-2}\right)$. (l) is an equicontour plot of $(\mathrm{j})$; line corresponds to $\left(0.5 \mathrm{FS}=7.0 \times 10^{13} \mathrm{~cm}^{-2}\right)$. (From laser spot \#2 of Fig. 3.2(b).) 


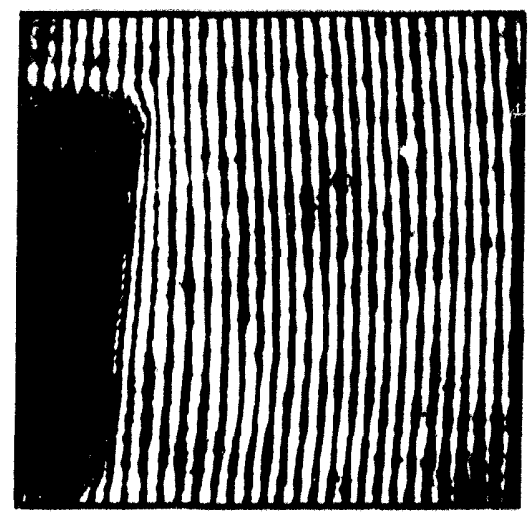

(m)

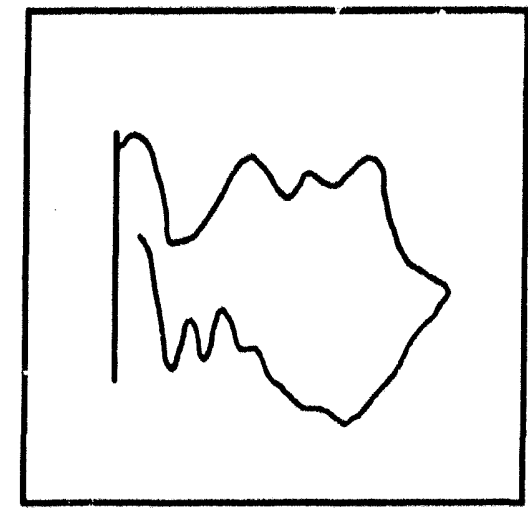

(n)

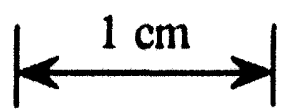

Fig. 4.24 Time scan in 1 Torr argon gas, continued. Hologram (m) taken at $\left(50.0 \mu \mathrm{s},-0.006 \mathrm{~nm}, \approx 2.4 \mathrm{~J} / \mathrm{cm}^{2}\right)$. (n) is an equicontour plot of $(\mathrm{m})$, line corresponds to $\left(0.5 \mathrm{FS}=7.0 \times 10^{13} \mathrm{~cm}^{-2}\right)$. (From laser spot \#2 of Fig. 3.2(b).j 


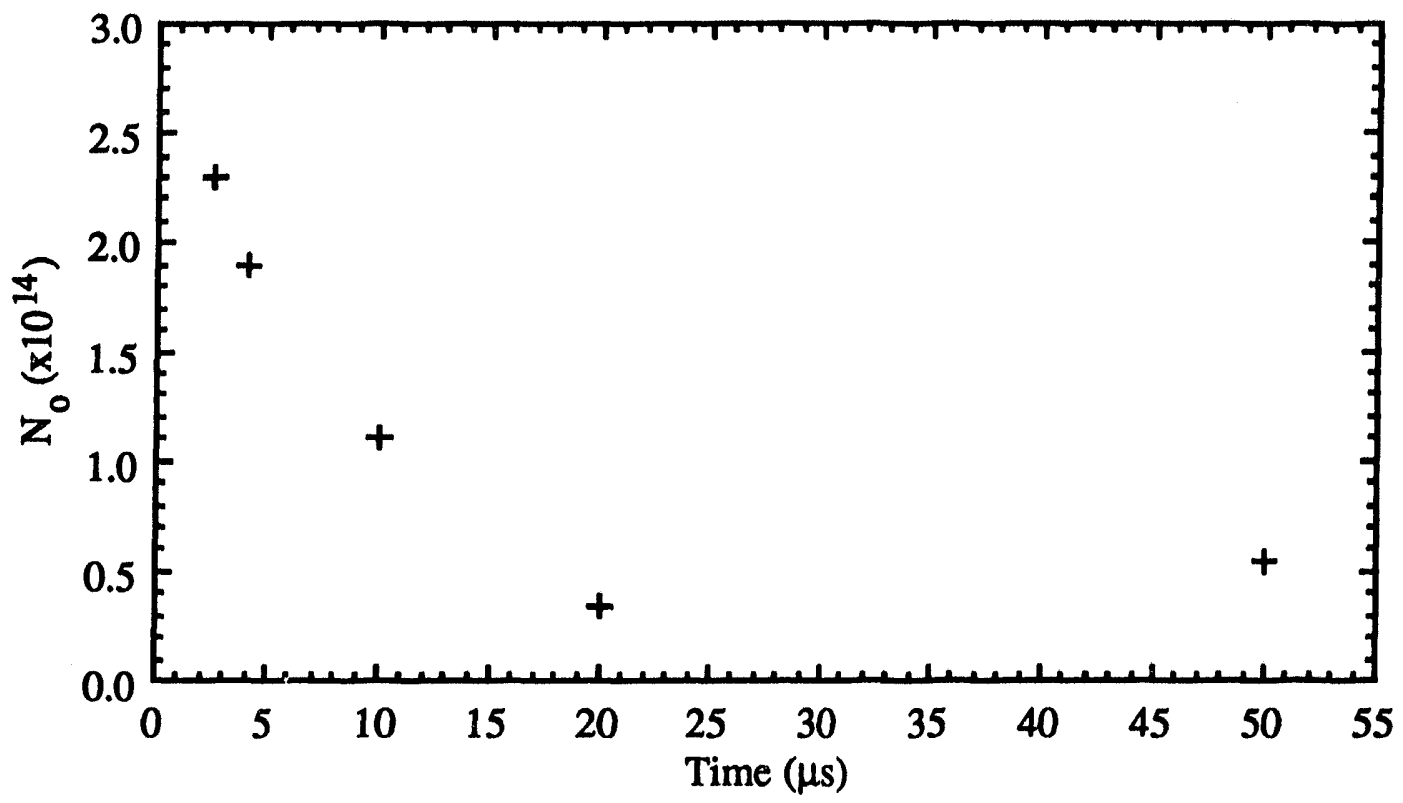

Fig. 4.25(a) Total number of aluminum neutral atoms, $\mathrm{N}_{\mathrm{o}}$, versus time in 1 Torr argon gas.

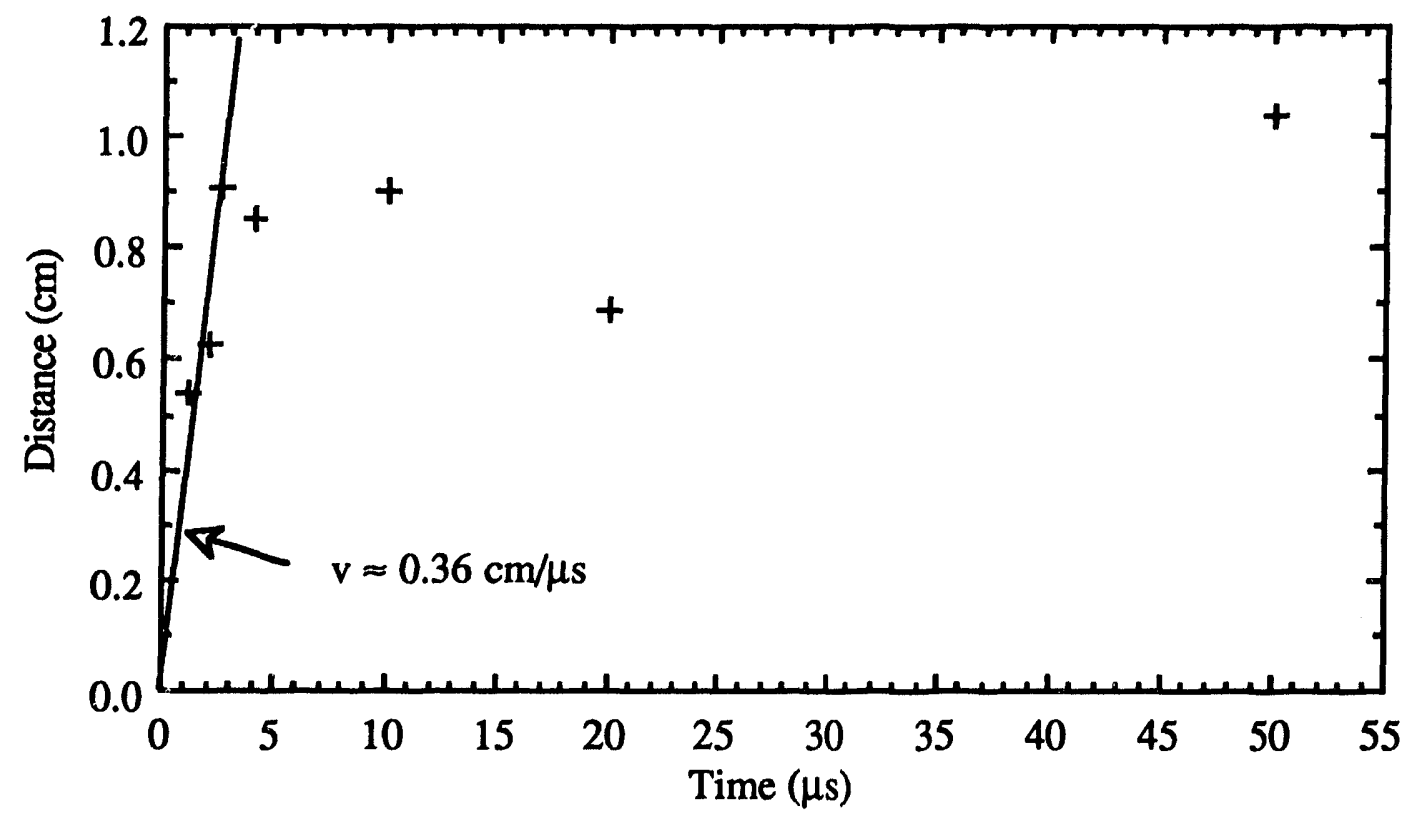

Fig. 4.25(b) Expansion of the $1 \times 10^{14} \mathrm{~cm}^{-2}$ equicontour line in 1 Torr argon gas. 
condensation of the atoms to dimers and to the fluid dynamics of one directionally expanding fluid in another (Sap92). Fourth, notice that the plume separates from the target by $20 \mu \mathrm{s}$. Finally, notice that the plume is observed as late as $50.0 \mu$ s with RHI at 1 Torr, while the latest time an ablation plume was observed in the previous vacuum data was $4.0 \mu \mathrm{s}$ in a DLRAP photo, which is more sensitive than RHI. Not only does this indicate collisions with the argon gas are holding back the expanding ablation plume, but also that the conditions are such in the plume to prevent significant condensation of the aluminum neutral atoms after that amount of time $(50 \mu \mathrm{s})$.

As with the holographic time scans at lower pressures, the peak line-density in the earliest holograms is obscured by absorption. The peak line-density at $2.50 \mu \mathrm{s}$ and $4.00 \mu \mathrm{s}$ is $6.0 \times 10^{14} \mathrm{~cm}^{-2}$, and it decreases to the detectability limit at this $|\Delta \lambda|, 7 \times 10^{13} \mathrm{~cm}^{-2}$, by $20.0 \mu \mathrm{s}$. These correspond to approximate aluminujm neutral densities of $\approx 7 \times 10^{14} \mathrm{~cm}^{-3}$ and $7 \times 10^{13} \mathrm{~cm}^{-3}$ for the earlier and later times, respectively.

Figure 4.25(a) shows the total number of aluminum neutral atoms counted for each hologram versus time. The value of $N_{0}, \approx 3 \times 10^{13}$ to $\approx 3 \times 10^{14}$, is similar to the values counted for the other time scans and the value clearly decreases with time as the aluminum atoms expand outward beyond the range of detection using RHI. Figure 4.25(b) shows the expansion of the $1 \times 10^{14} \mathrm{~cm}^{-2}$ equicontour as a function of time. The expansion velocity of $0.36 \mathrm{~cm} / \mu \mathrm{s}$ is nearly half the slowest expansion velocity for the vacuum and lower pressure time scans $(0.77 \mathrm{~cm} / \mu \mathrm{s})$. The expansion of the $1 \times 10^{14} \mathrm{~cm}^{-2}$ equicontour then appears to more or less stabilize at about $1 \mathrm{~cm}$ between $5 \mu$ s and $50 \mu \mathrm{s}$. 
Figure 4.26 shows a RHI time scan in 1 Torr argon RF-plasma. The RF-plasma was produced between two parallel plates enclosing the region of plume expansion, as described in Chapter 3. The argon plasma is assumed to have a maximum electron density of $1 \times 10^{10} \mathrm{~cm}^{-3}$ and a maximum kinetic temperature of $2 \mathrm{eV}$ (Bro93). The Stark broadening introduced into the aluminum neutral line-density calculations due to the RF-plasma is minimal for the given electron density and temperature. Therefore, the calculations in Chapter 2 assuming no Stark broadening are used here for the plasma case as well.

The first thing to notice is that the plume shapes look very similar to the non-plasma case. Secondly, note the separation of the expanding plume and the residual plume at $20.0 \mu \mathrm{s}$ in Fig. 4.26(1) and only the expanding plume at $50.0 \mu \mathrm{s}$ in Fig. 4.26(n). Third, the counted number of aluminum atoms in the plume is slightly less for the RF-plasma case than for the non-plasma case, as shown in Fig. 4.27(a). Fourth, the $1 \times 10^{14} \mathrm{~cm}^{-2}$ equicontour line appears to expand farther away from the target in the RF-plasma case than in the gas case.

At this time, it is unknown whether the observed differences are: 1) just shot-to-shot, statistical variations of the ablation plumes; 2) caused by the RF electric field directly affecting the ablated aluminum ions at very early times $(<0.1 \mu \mathrm{s})$; or 3 ) caused by collisions between the ablation plume and the energetic electrons and argon ions during the entire expansion time. For example, energetic collisions of the argon plasma with the ablation plume could cause either ionization or condensation of the aluminum atoms, thus causing the observed reduction in the number of neutral atoms. Since this issue is the focal point of Chi Hong Ching's dissertation, which is forthcoming, Fig. 4.26 is shown here as an example 


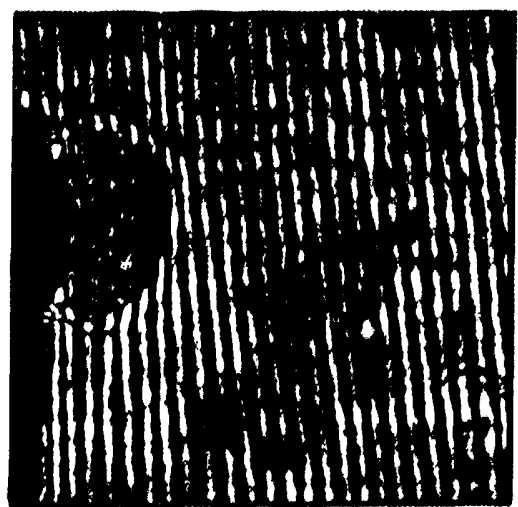

(a)

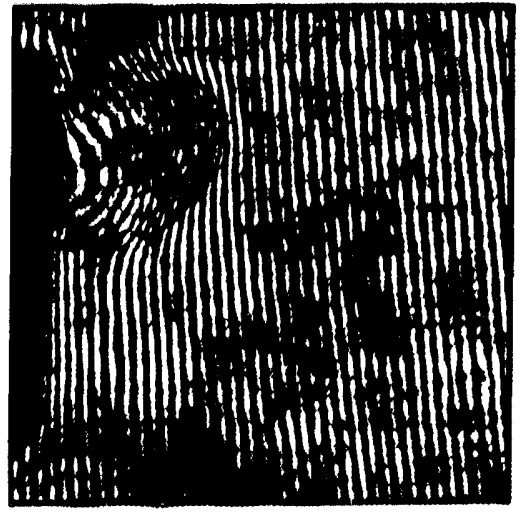

(b)

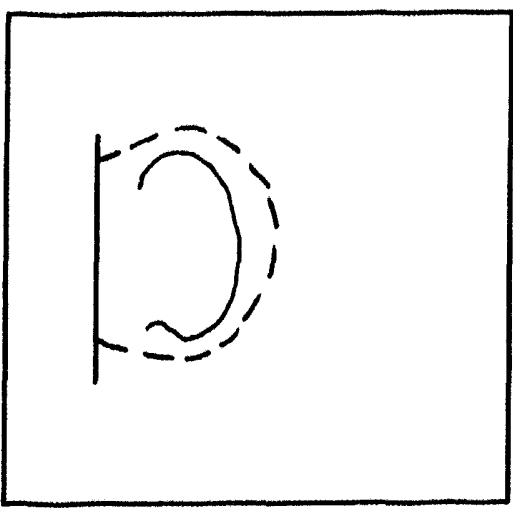

(c)

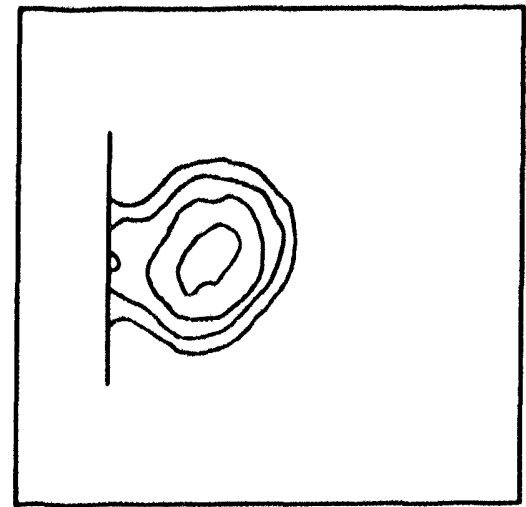

(d)

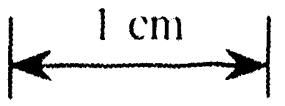

Fig. 4.26 Time scan in 1 Torr argon RF/plasma. Hologram (a) taken at $\left(1.00 \mu \mathrm{s},-0.006 \mathrm{~nm}, \approx 2.5 \mathrm{~J} / \mathrm{cm}^{2}\right)$ and hologram (b) taken at $(2.00$ $\mu \mathrm{s},-0.006 \mathrm{~nm}, \approx 2.3 \mathrm{~J} / \mathrm{cm}^{2}$ ). (c) is an equicontour plot of (a), dashed line corresponds to a shock wave and solid line corresponds to (0.5 FS $\left.=7.0 \times 10^{13} \mathrm{~cm}^{-2}\right)$; (d) is an equicontour plot of (b), lines co. respond to increasing fringe shifts, from outer to inner line: $\left(1.0 \mathrm{FS}=1.4 \times 10^{14}\right.$ $\left.\mathrm{cm}^{-2}\right),,\left(2.0 \mathrm{FS}=2.9 \times 10^{14} \mathrm{~cm}^{-2}\right),\left(3.0 \mathrm{FS}=4.4 \times 10^{14} \mathrm{~cm}^{-2}\right)$, and (4.0 FS $=6.0 \times 10^{14} \mathrm{~cm}^{-2}$ ). (From laser spot \#2 of Fig. 3.2(b).) 


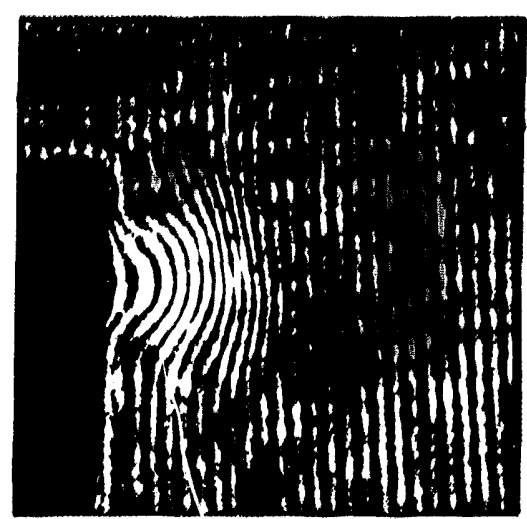

(e)

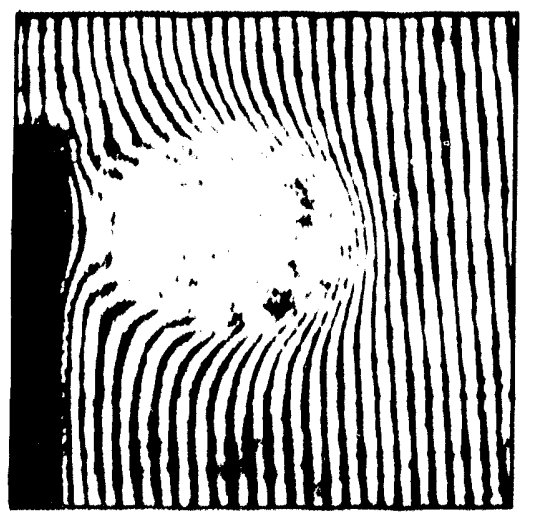

(f)

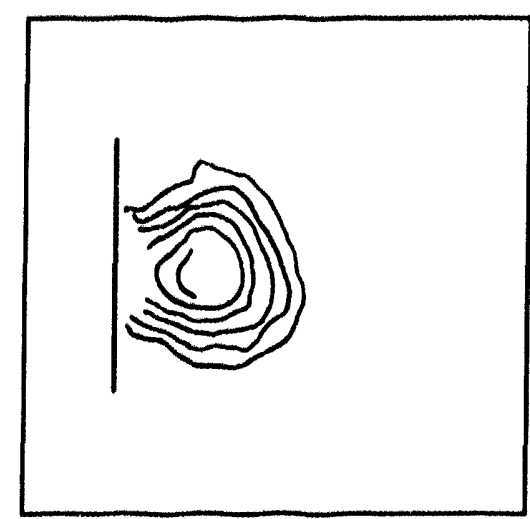

(g)

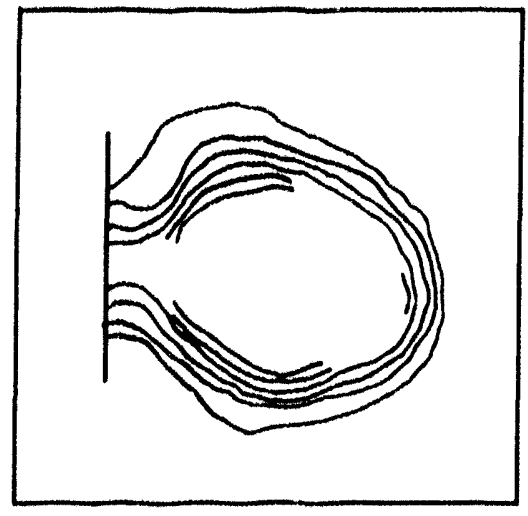

(h)

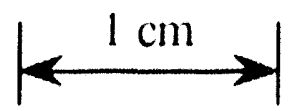

Fig. 4.26 Time scan in 1 Torr argon RF/plasma, continued.

Hologram (e) taken at $\left(2.50 \mu \mathrm{s},-0.006 \mathrm{~nm}, \approx 2.1 \mathrm{~J} / \mathrm{cm}^{2}\right)$ and hologram (f) taken at $\left(4.02 \mu \mathrm{s},-0.006 \mathrm{~nm}, \approx 2.4 \mathrm{~J} / \mathrm{cm}^{2}\right)$. (g) is an equicontour plot of (e), and (h) is an equicontour plot of (f); lines correspond to increasing fringe shifts, from outer to inner line: $\left(0.5 \mathrm{FS}=7.0 \times 10^{13}\right.$ $\left.\mathrm{cm}^{-2}\right),\left(1.0 \mathrm{FS}=1.4 \times 10^{14} \mathrm{~cm}^{-2}\right),\left(1.5 \mathrm{FS}=2.1 \times 10^{14} \mathrm{~cm}^{-2}\right),(2.0 \mathrm{FS}$ $\left.=2.9 \times 10^{14} \mathrm{~cm}^{-2}\right),\left(2.5 \mathrm{FS}=3.6 \times 10^{14} \mathrm{~cm}^{-2}\right)$ and $\left(3.0 \mathrm{FS}=4.4 \times 10^{14} \mathrm{~cm}^{-2}\right)$. (From laser spot \#2 of Fig. 3.2(b).) 


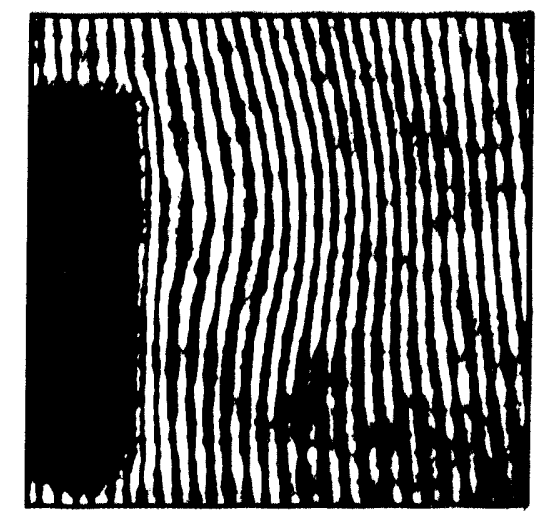

(i)

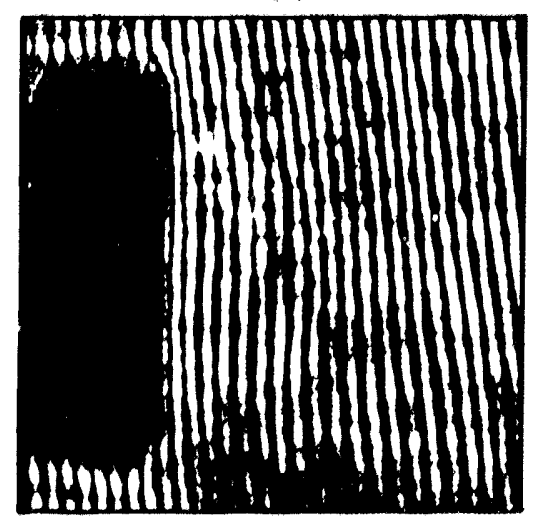

(j)

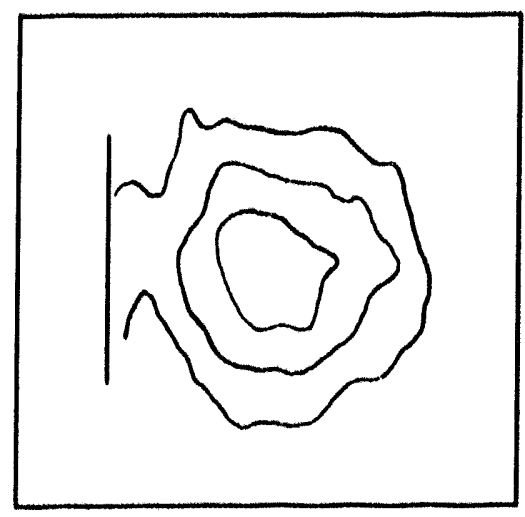

(k)

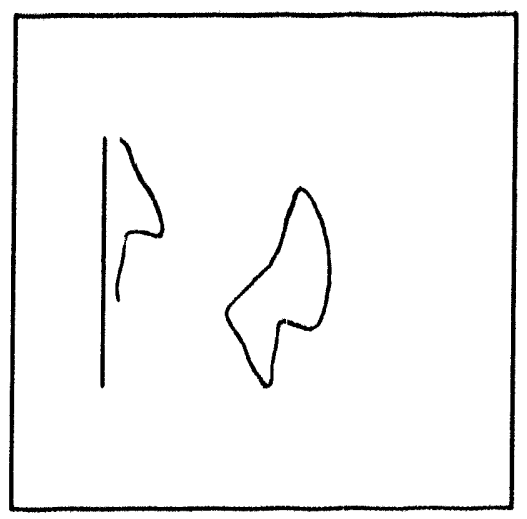

(1)

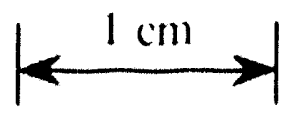

Fig. 4.26 Time scan in 1 Torr argon Rl/plasma, continued.

Hologram (i) taken at $\left(10.0 \mu \mathrm{s},-\left(0.006 \mathrm{~nm}, \approx 2.4 \mathrm{~J} / \mathrm{cm}^{2}\right)\right.$ and hologram (j) taken at $\left(20.0 \mu \mathrm{s},-\left(0.006 \mathrm{~nm}, \approx 2.4 \mathrm{~J} / \mathrm{cm}^{2}\right)\right.$. (k) is an equicontour plot of (i), lines correspond to increasing fringe shifts, from outer to inner line: $\left(0.5 \mathrm{FS}=7.0 \times 10^{13} \mathrm{~cm}^{-2}\right),\left(1.0 \mathrm{FS}=1.4 \times 10^{14} \mathrm{~cm}^{-2}\right)$, and $(1.5 \mathrm{FS}$ $\left.=2.1 \times 10^{14} \mathrm{~cm}^{-2}\right)$. (l) is an equicontour plot of $(\mathrm{j})$; line corresponds to $\left(0.5 \mathrm{FS}=7.0 \times 10^{13} \mathrm{~cm}^{-2}\right.$ ). (From laser spot $\# 2$ of $\mathrm{ligg} .3 .2(\mathrm{~b})$.) 


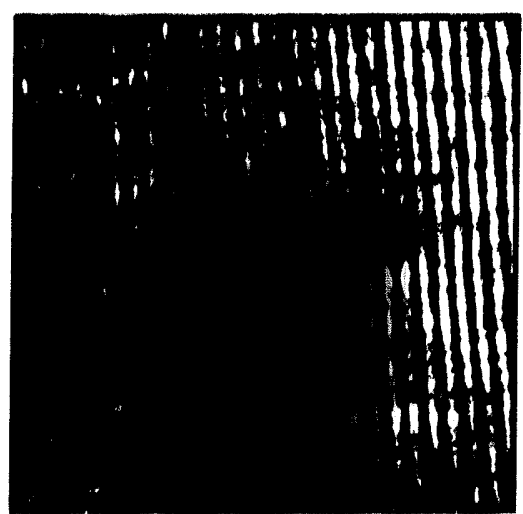

(m)

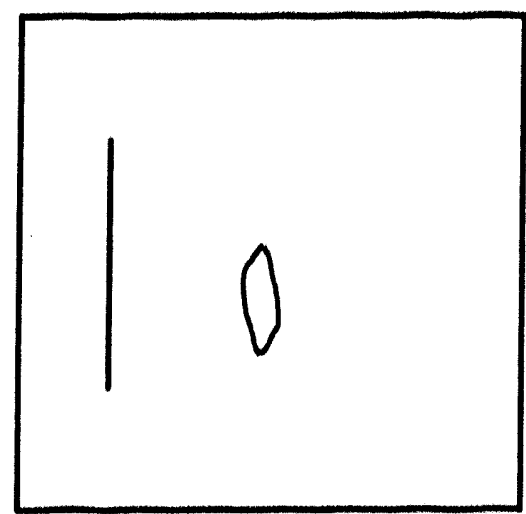

(n)

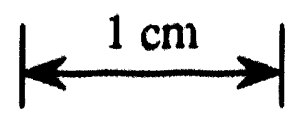

Fig. 4.26 Time scan in 1 Torr argon RF/plasma, continued. Hologram $(\mathrm{m})$ taken at $\left(50.0 \mu \mathrm{s},-0.006 \mathrm{~nm}, \approx 2.4 \mathrm{~J} / \mathrm{cm}^{2}\right)$. (n) is an equicontour plot of $(\mathrm{m})$, line corresponds to $\left(0.5 \mathrm{FS}=7.0 \times 10^{13} \mathrm{~cm}^{-2}\right)$. (From laser spot \#2 of Fig. 3.2(b).) 


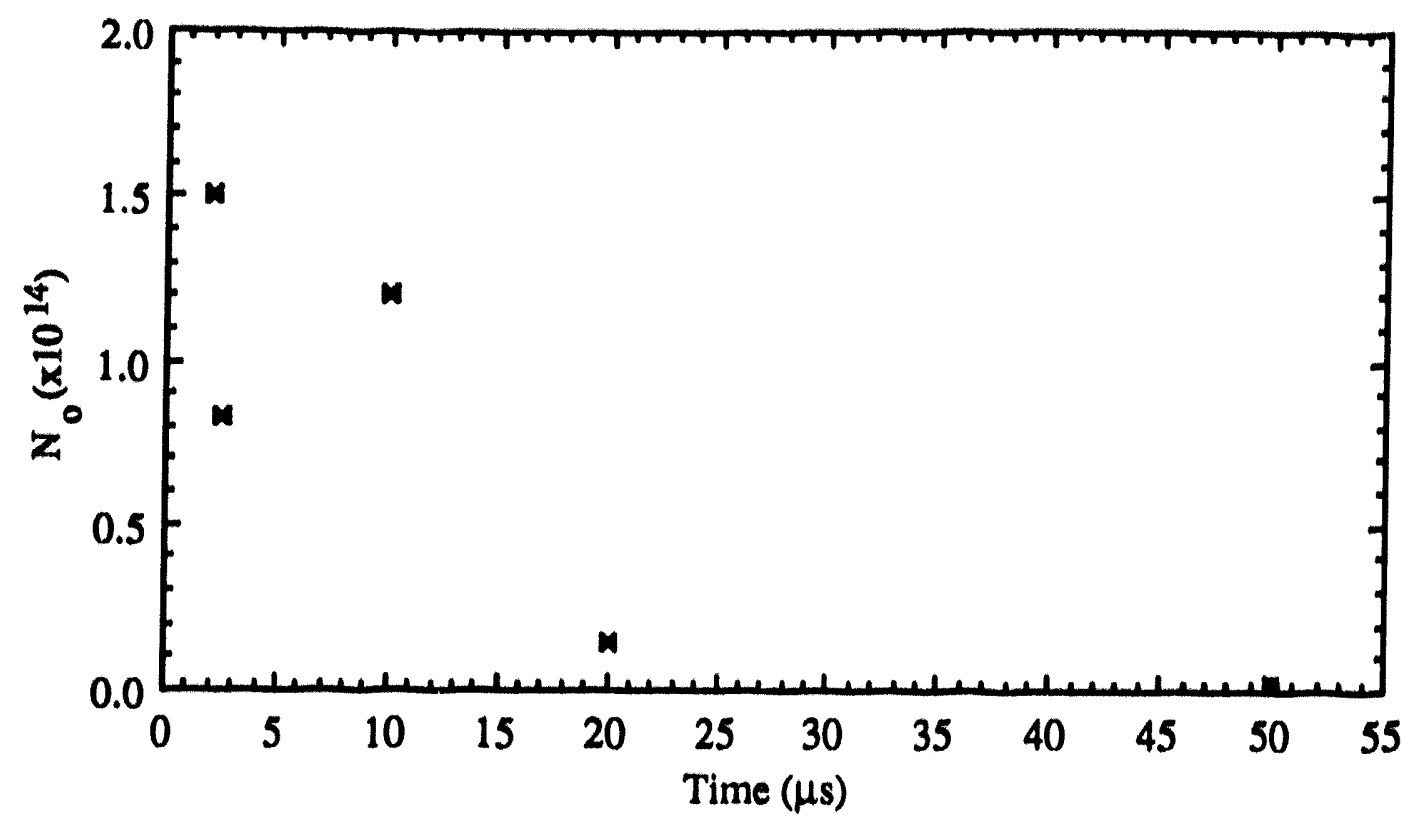

Fig. 4.27(a) Total number of aluminum neutral atoms, $N_{0}$, versus time in 1 Torr argon RF/plasma.

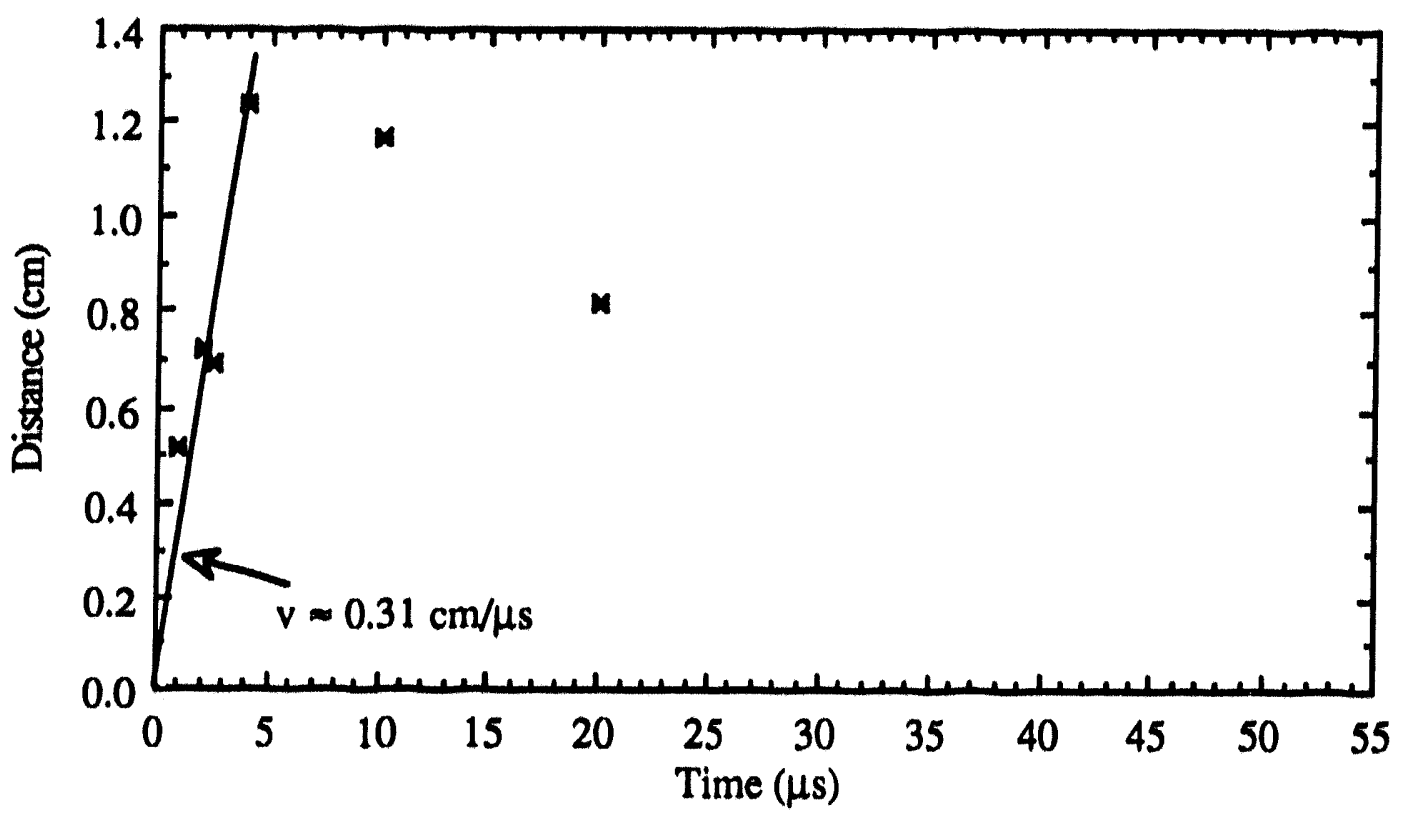

Fig. 4.27(b) Expansion of the $1 \times 10^{14} \mathrm{~cm}^{-2}$ equicontour line in 1 Torr argon RF/plasma. 
of the copious laser-ablation-plume-expansion-in-plasma-environments data by which this author has demonstrated the applicability of RHI.

Figure 4.28 shows an early time scan at a background argon gas pressure of 35 Torr. Although a complete time scan at this pressure is not presented, the holograms show two interesting effects. First of all, this is the only pressure at which non-resonant fringe shift was observed and secondly, the plume expansion is even slower than at 1 Torr. Although the Lorentz broadening mechanisms become on the order of the natural broadening for a background pressure of 35 Torr, the effect of the overall broadening induced onto the calculation of line-densities is minimal. Therefore, the calculations of aluminum neutral line-density interpretation in Chapter 2, which assume no Lorentz broadening, are utilized in the interpretation of the 35 Torr data.

The only nonresonant fringe shift was observed at very early times, $0.2 \mu \mathrm{s}$ and $0.5 \mu \mathrm{s}$. Figures $4.28(\mathrm{a})$ and (b) show a near-resonant and a non-resonant hologram, respectively, taken at $0.22 \mu \mathrm{s}$. Similarly, Figures 4.28(e) and ( $f$ ) show a near-resonant and a non-resonant hologram, respectively, taken at $0.52 \mu \mathrm{s}$. The resonant-only fringe shift for each time was found by overlaying the two traces for the same time and subtracting the fringe shifts at each spatial location. Figures $4.28(i)$ and (j) are therefore the resulting subtractions at $0.22 \mu \mathrm{s}$ and $0.52 \mu \mathrm{s}$, respectively, and represent the resonant-only fringe shift due to interaction with aluminum neutral atoms. Figures $4.28(\mathrm{k})$ to (n) show holograms and their tracings at $1.80 \mu \mathrm{s}$ and $3.84 \mu \mathrm{s}$. There was no fringe shift observed at the off-resonant wavelength, so all of the observed fringe shift is due to resonant effects.

The total number of aluminum neutral atoms counted at $0.22 \mu \mathrm{s}$ is 


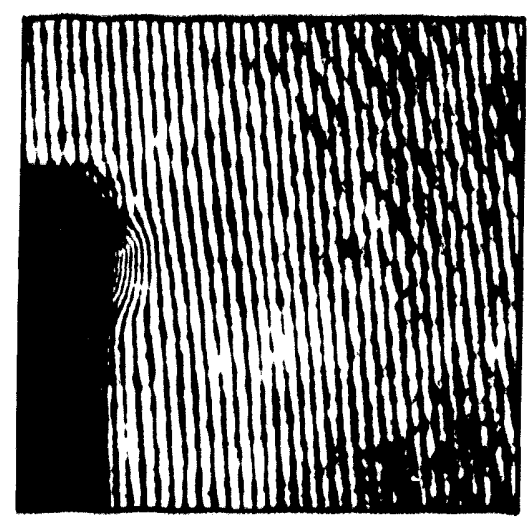

(a)

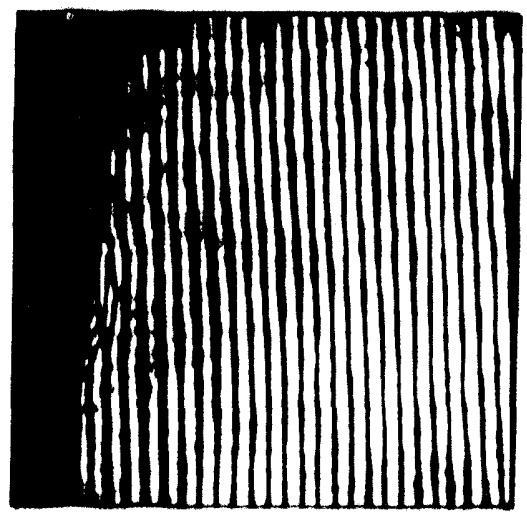

(b)

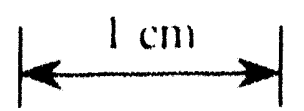

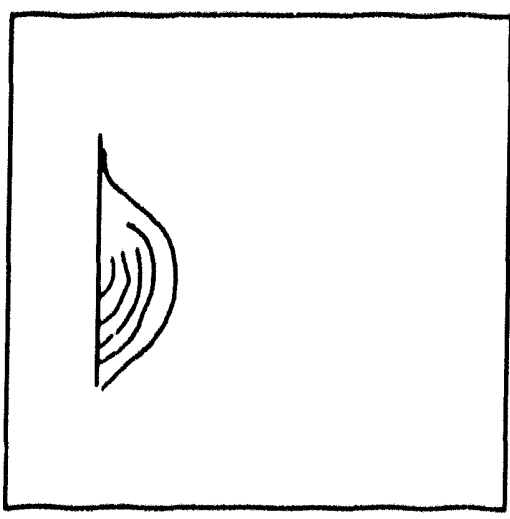

(c)

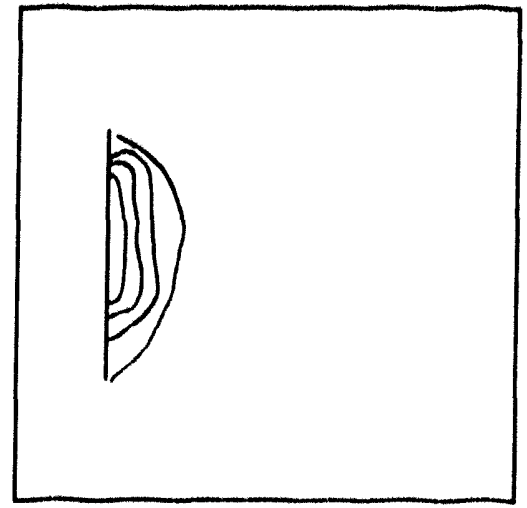

(d)

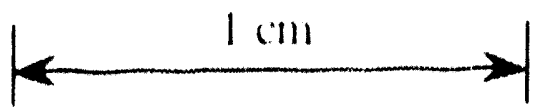

Fig. 4.28 Time scan in 3.5 'Torr argon gas. Hologram (a), taken at $\left(0.22 \mu \mathrm{s},+0.004 \mathrm{~nm}, \approx 2.4 \mathrm{~J} / \mathrm{cm}^{2}\right)$, is the near-resonance hologram for $0.22 \mu \mathrm{s}$. Hologram (b), taken at $\left(0.22 \mu \mathrm{s},+0.503 \mathrm{~nm}, \approx 2.4 \mathrm{~J} / \mathrm{cm}^{2}\right)$, is the off-resonance hologram for $0.22 \mu \mathrm{s}$. (c) is an equicontour plot of (a), lines correspond to increasing fringe shifts, from outer to inner line: (1.0 FS), (2.0 FS), (3.0 FS), (4.0 FS), and (5.0 FS); (d) is an equicontour plot of (b), lines correspond to increasing fringe shifts, from outer to inner line: (0.5 FS), (1.0 FS), (1.5 FS), (2.0 FS). The subtraction of (d) from (c) is shown in (i). (From laser spot \#2 of Fig. $3.2(h)$.) 


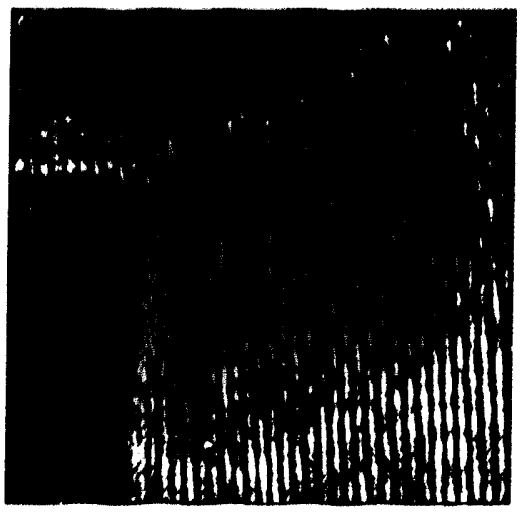

(e)

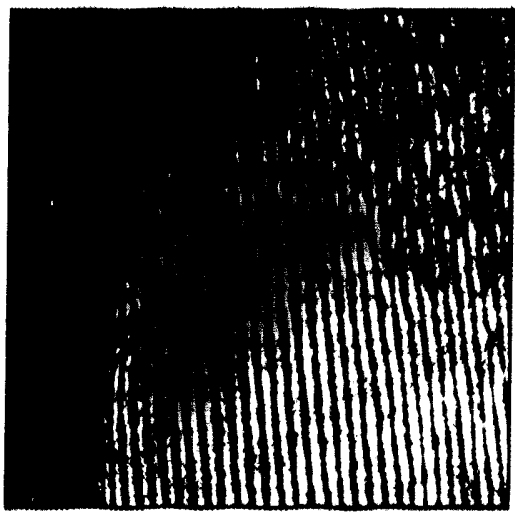

(f)

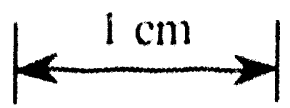

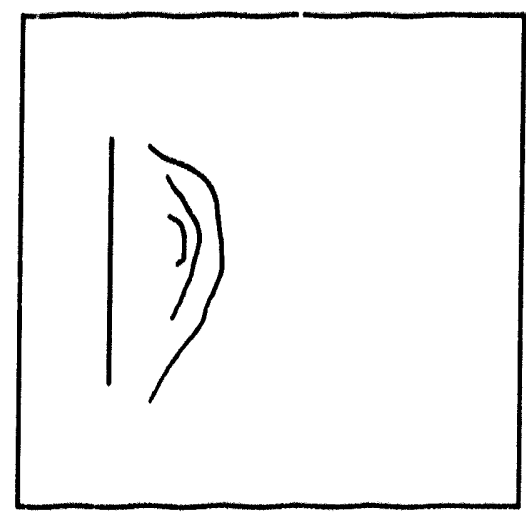

(g)

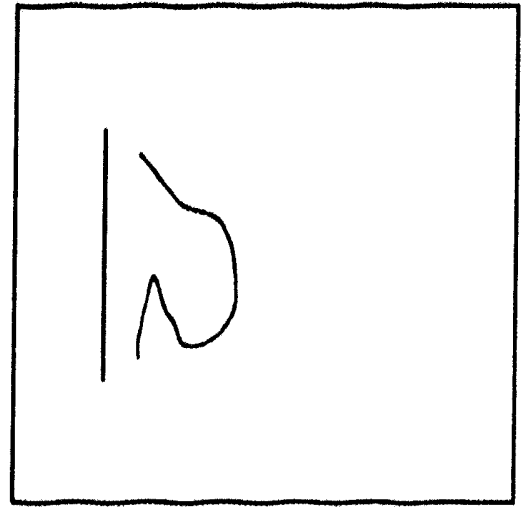

(h)

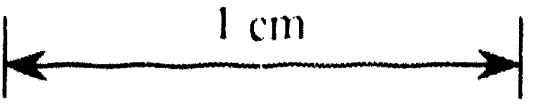

Fig. 4.28 Time scan in 35 Torr argon gas, continued. Hologram (e), taken at $\left(0.52 \mu \mathrm{s},+0.004 \mathrm{~nm}, \approx 2.4 \mathrm{~J} / \mathrm{cm}^{2}\right)$, is the near-resonance hologram for $0.52 \mu \mathrm{s}$. Hologram $(\mathrm{f})$, taken at $(0.52 \mu \mathrm{s},+0.503 \mathrm{~nm}$, $\approx 2.4 \mathrm{~J} / \mathrm{cm}^{2}$ ), is the off-resonance hologram for $0.52 \mu \mathrm{s}$. (g) is an equicontour plot of (e), lines correspond to increasing fringe shifts, from outer to inner line: (0.5 FS), (1.0 FS) and (1.5 FS); (h) is an equicontour plot of $(f)$, line corresponds to $(0.5 \mathrm{FS})$. The subtraction of $(h)$ from $(g)$ is shown in (j). (From laser spot \#2 of Fig. 3.2(b).) 


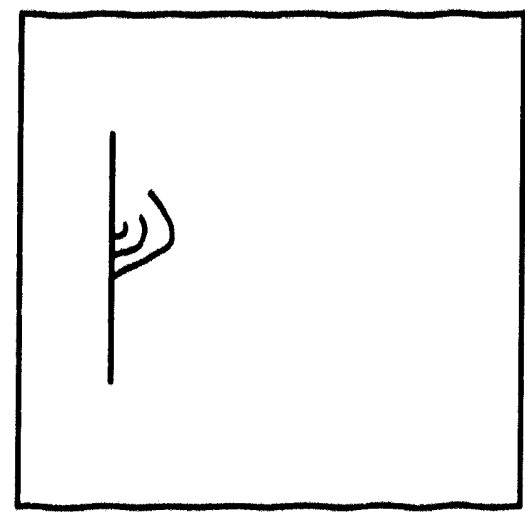

(i)

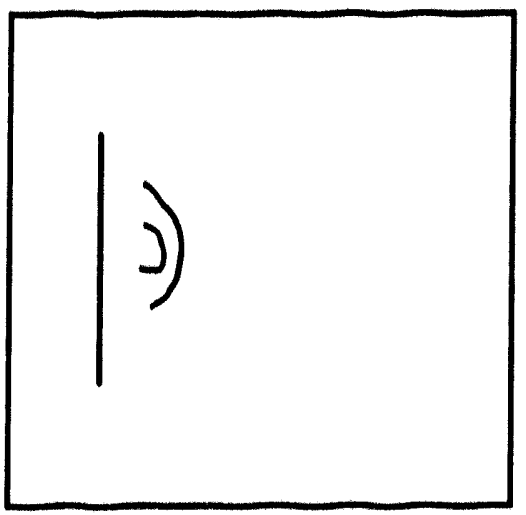

(j)

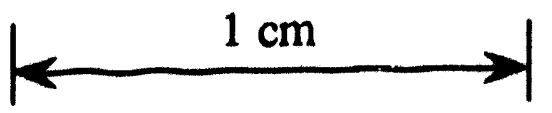

Fig. 4.28 Time scan in 35 Torr argon gas, continued. Tracing (i) is the point-to-point subtraction of (d) from (c) and represents the resonant-only fringe shift at $0.22 \mu \mathrm{s}$. Lines correspond to increasing fringe shifts, from outer to inner line: $\left(1.0 \mathrm{FS}=9.0 \times 10^{13} \mathrm{~cm}^{-2}\right)$, $\left(2.0 \mathrm{FS}=1.9 \times 10^{14} \mathrm{~cm}^{-2}\right)$, and $\left(3.0 \mathrm{FS}=3.0 \times 10^{14} \mathrm{~cm}^{-2}\right)$. Tracing $(j)$ is the point-to-point subtraction of $(\mathrm{h})$ from $(\mathrm{g})$ and represents the resonant-only fringe shift at $0.52 \mu \mathrm{s}$. Lines correspond to increasing fringe shifts, from outer to inner line: $\left(0.5 \mathrm{FS}=4.3 \times 10^{13} \mathrm{~cm}^{-2}\right)$ and $\left(1.0 \mathrm{FS}=9.0 \times 10^{13} \mathrm{~cm}^{-2}\right.$ ). (From laser spot \#2 of Fig. 3.2(b).) 


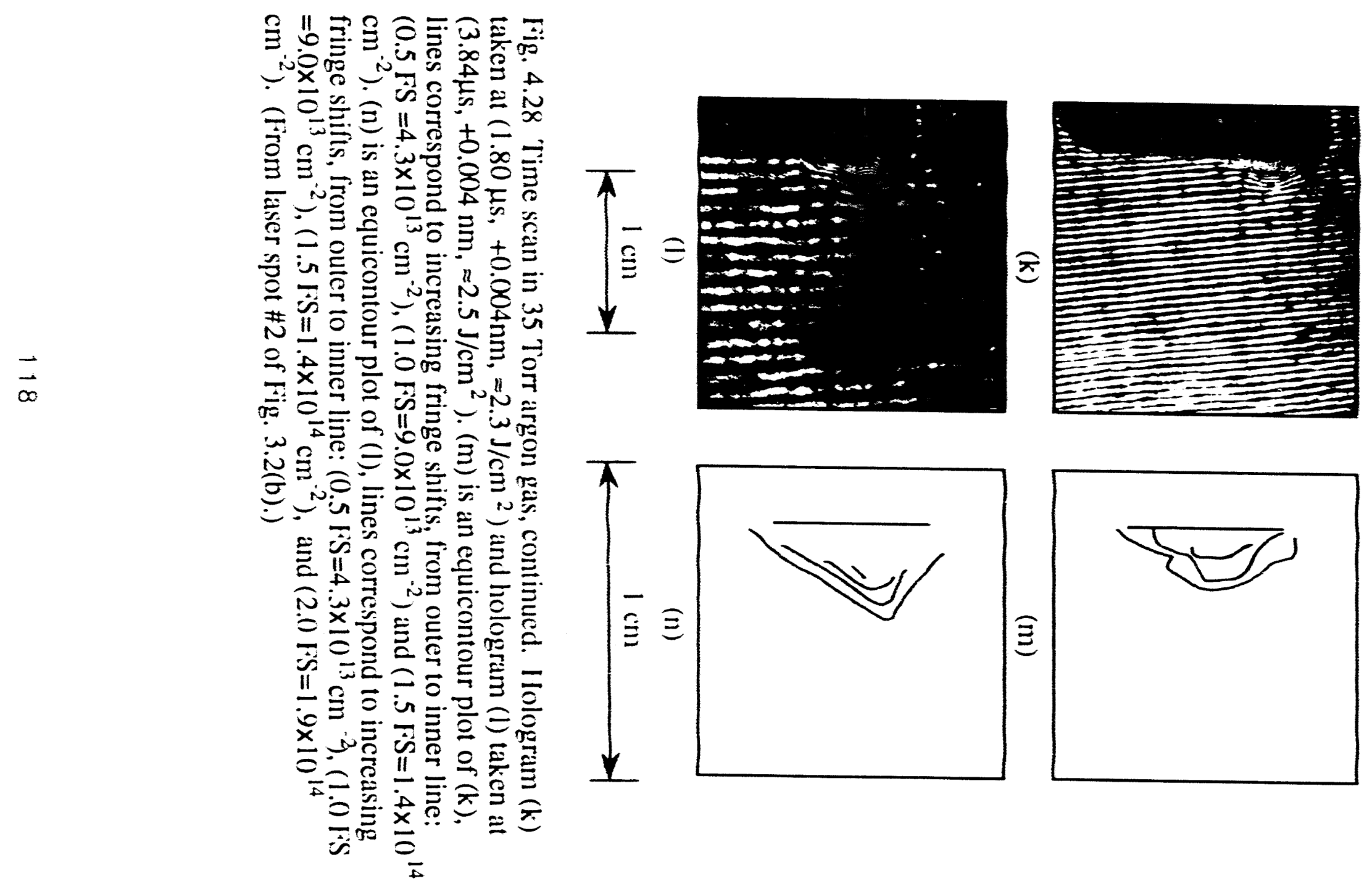




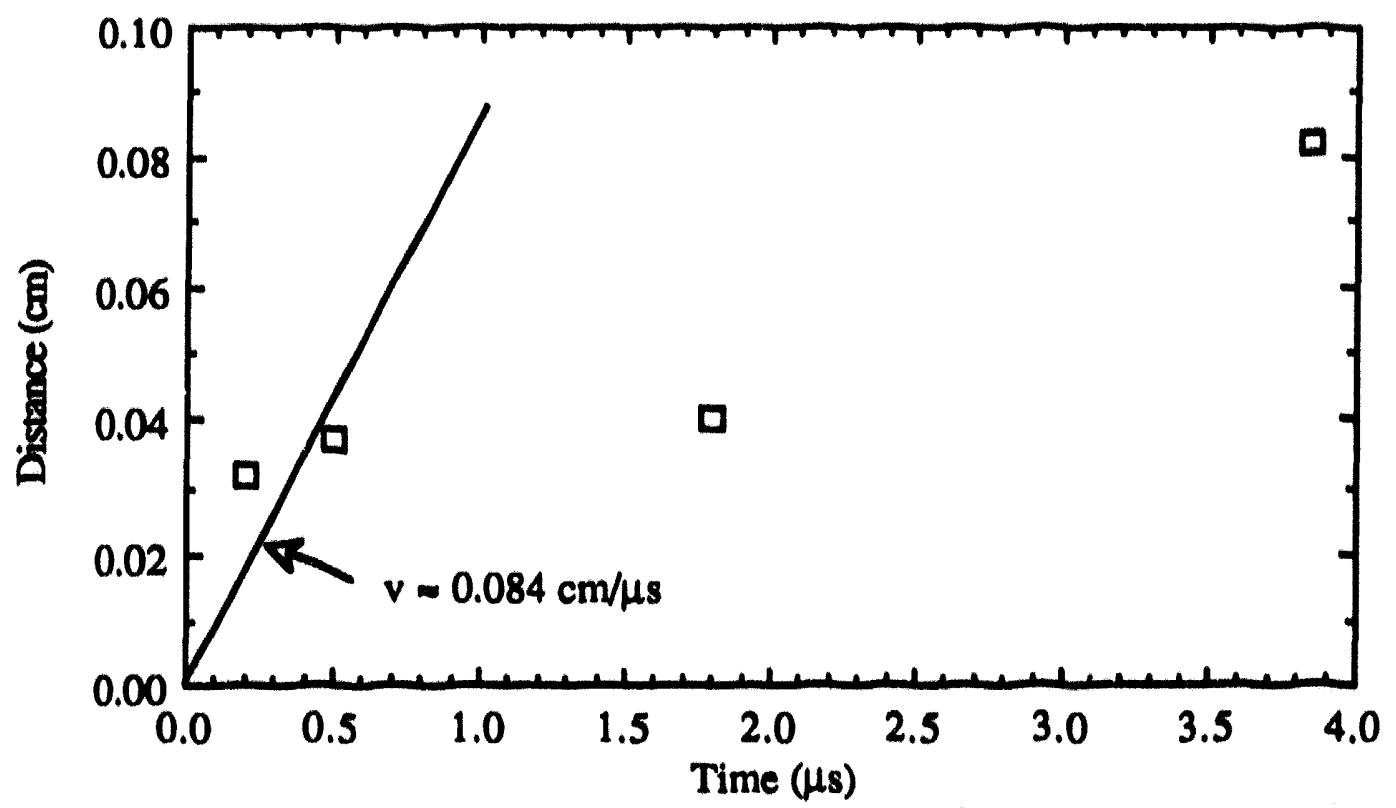

Fig. 4.29 Expansion of the $1 \times 10^{14} \mathrm{~cm}^{-2}$ equicontour line in 35 Torr argon gas.

$2.5 \times 10^{12} \mathrm{~cm}^{-2}$. The value of $\mathrm{N}_{0}$ is an order of magnitude smaller than the values of No determined for previous time scans. This is primarily due to the inadequate spatial resolution of the holograms at early times when the plume is very close to the target surface and could also be due to forced recondensation on the target at these pressures. It is suggested that a hologram at a much later time, such as $20 \mu$ s, would provide a much better count of the total number of ablated atoms in the plume.

Figure 4.29 shows the expansion of the $1 \times 10^{14} \mathrm{~cm}^{-2}$ equicontour. The initial expansion velocity of $\approx 0.084 \mathrm{~cm} / \mu \mathrm{s}$ is about 4 times smaller than the expansion velocities measured at 1 Torr and the expansion velocity decreases after $0.5 \mu \mathrm{s}$ to about $0.01 \mathrm{~cm} / \mu \mathrm{s}$.

Fig. 4.30 shows the total number of aluminum neutral atoms in the ground state measured for all eight time scans; the values range from $2.5 \times 10^{12}$ to $3.2 \times 10^{14}$. Since many of the earlier No's in each time scan 


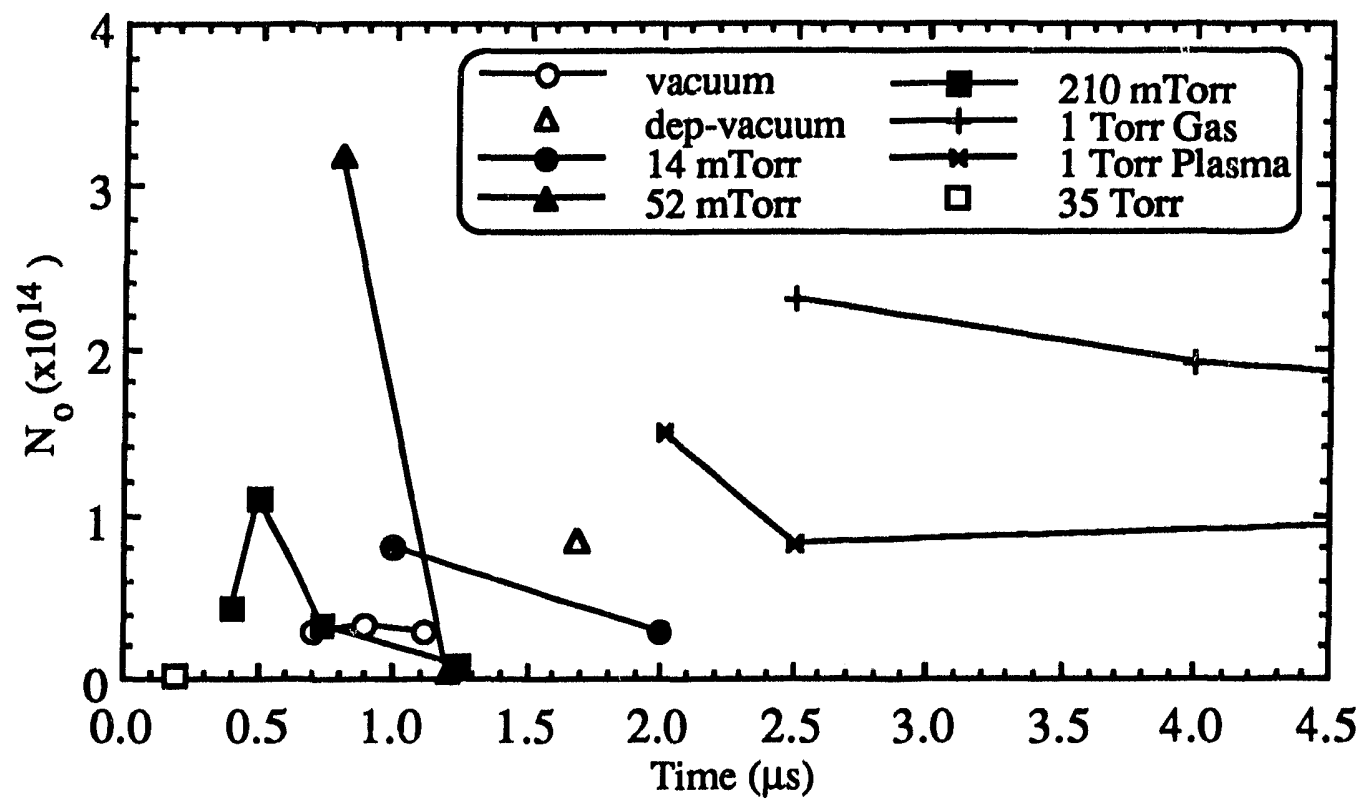

Fig. 4.30(a) Total number of aluminum neutral atoms, $\mathbf{N}_{0}$, versus time in vacuum and argon background gas; early times.

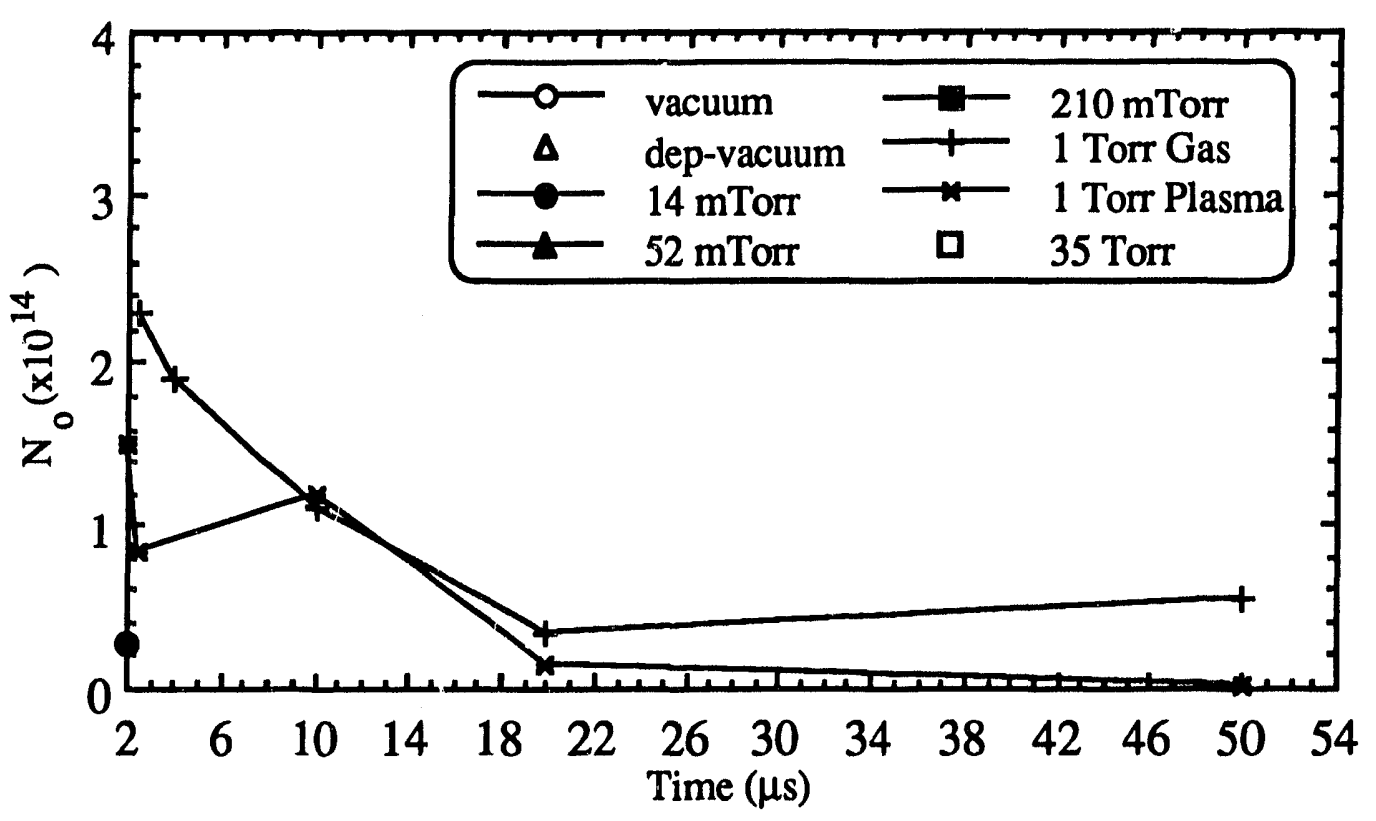

Fig. 4.30(b) Total number of aluminum neutral atoms, $\mathbf{N}_{\mathrm{o}}$, versus time in vacuum and argon background gas; later times. 
are $>1 \times 10^{14}$ and since $3 \times 10^{14}$ was the estimated maximum number of particles in the laser ablation deposition discussion above, $N_{O} \approx 3 \times 10^{14}$ is a good estimate of the number of aluminum ground-state neutral atoms that the $\mathrm{KrF}$ laser is ablating from the aluminum targets for the experimental parameiers in this work.

Resonant refraction is very strong, allowing only the edges of the ablation plume to be analyzed. The peak line-density for a given hologram was $3 \times 10^{14} \mathrm{~cm}^{-2}$ at $0.22 \mu \mathrm{s}$ and $2 \times 10^{14} \mathrm{~cm}^{-2}$ at $3.84 \mu \mathrm{s}$. Considering the small size of the plume, these correspond to particle densities of $1 \times 10^{15} \mathrm{~cm}^{-3}$ and $6 \times 10^{14} \mathrm{~cm}^{-3}$, respectively.

Figure 4.31 shows the expansion of the $1 \times 10^{14} \mathrm{~cm}^{-2}$ equicontour line for aluminum neutrals in 1 Torr argon gas and plasma, 35 Torr argon gas, and the deposition-in-vacuum plume expansion case for comparison. Notice that the plume expansion is significantly slowed for the 1 Torr case and even more so for the 35 Torr case. The approximate expansion velocities for all of the time scans are compiled in Figure 4.32 versus pressure. For $210 \mathrm{mTorr}$ and less, the expansion velocity is in the range $1.0 \mathrm{~cm} / \mu \mathrm{s}-1.4 \mathrm{~cm} / \mu \mathrm{s}$, while for higher background pressures of argon the expansion velocity decreases to as low as 0.084 for the 35 Torr case. 


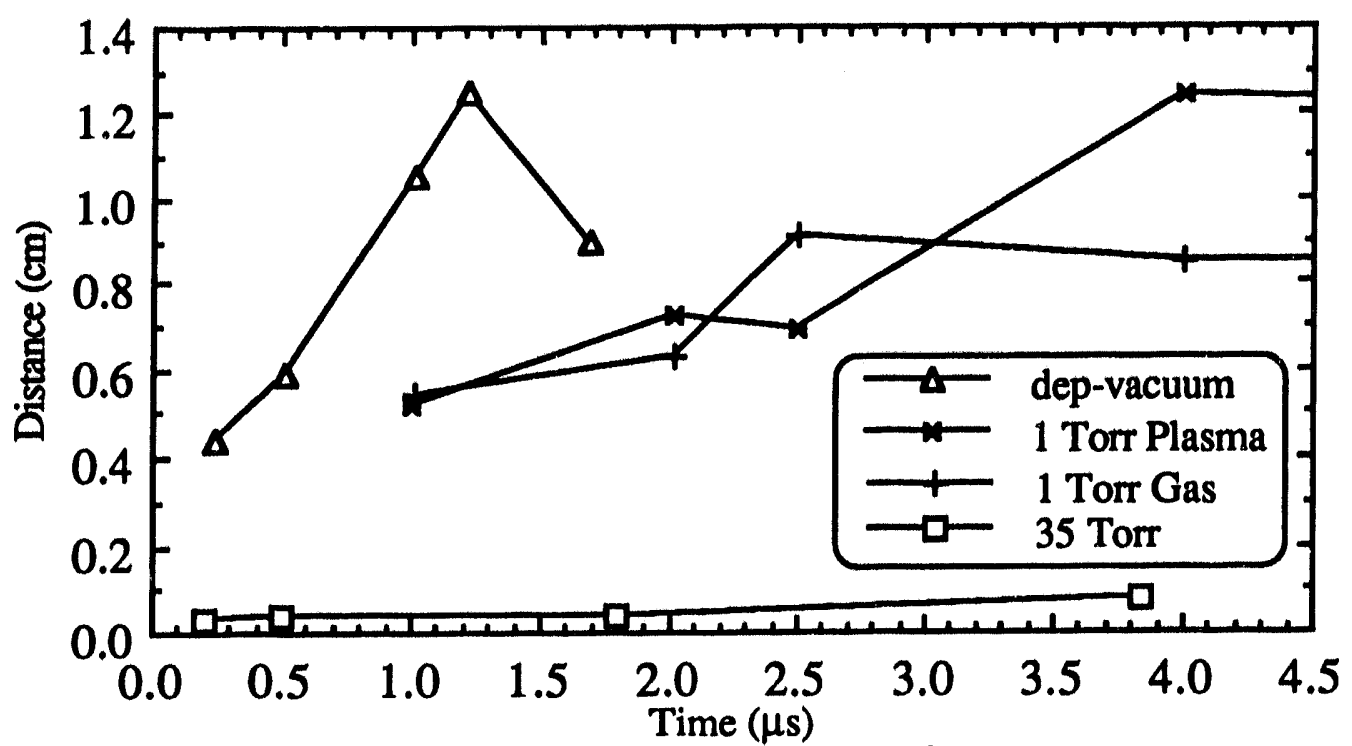

Fig. 4.31(a) Expansion of the $1 \times 10^{14} \mathrm{~cm}^{-2}$ equicontour line in 1 Torr and 35 Torr argon; early times. Deposition in vacuum expansion shown for comparison (from fig. 4.18(b)).

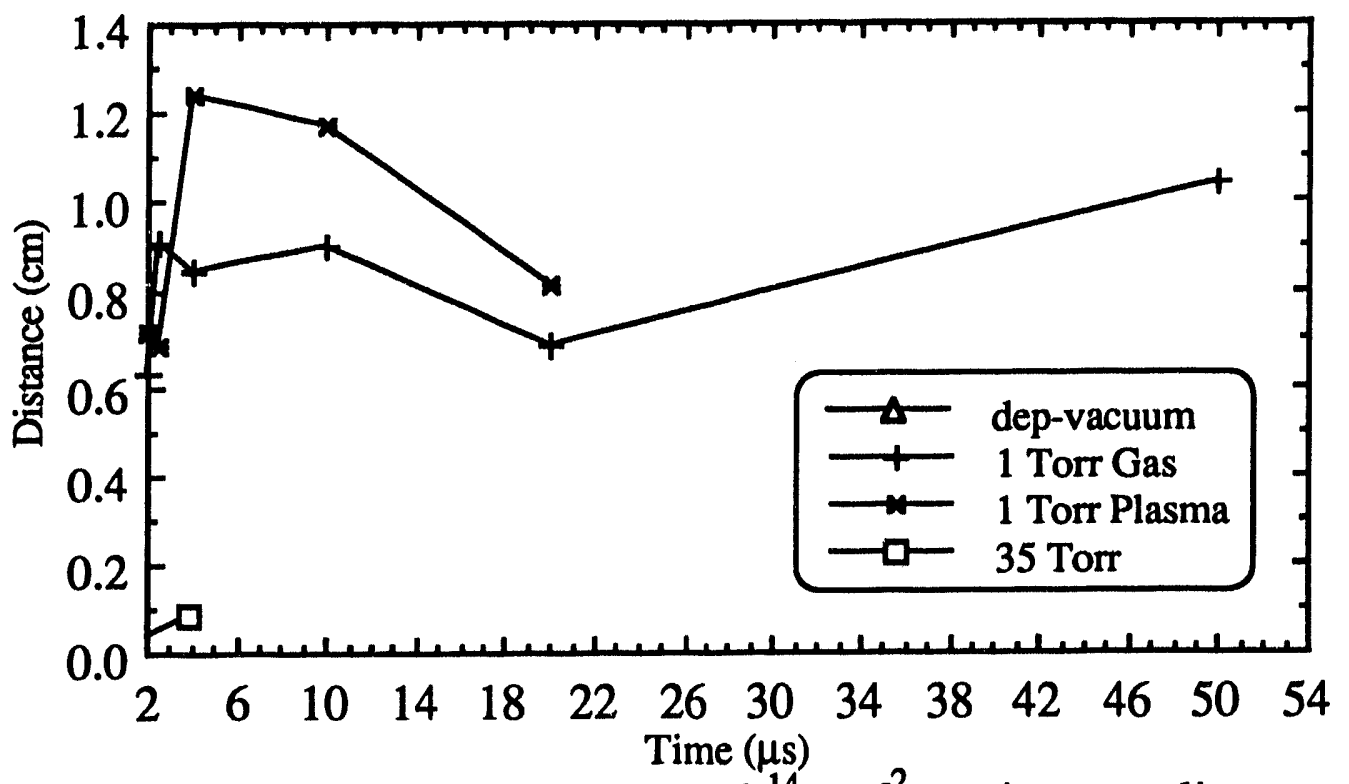

Fig. 4.31(b) Expansion of the $1 \times 10^{14} \mathrm{~cm}^{-2}$ equicontour line in 1 T'orr and 35 Torr argon; later times. Deposition in vacuum expansion shown for comparison (from fig. 4.18(b)). 


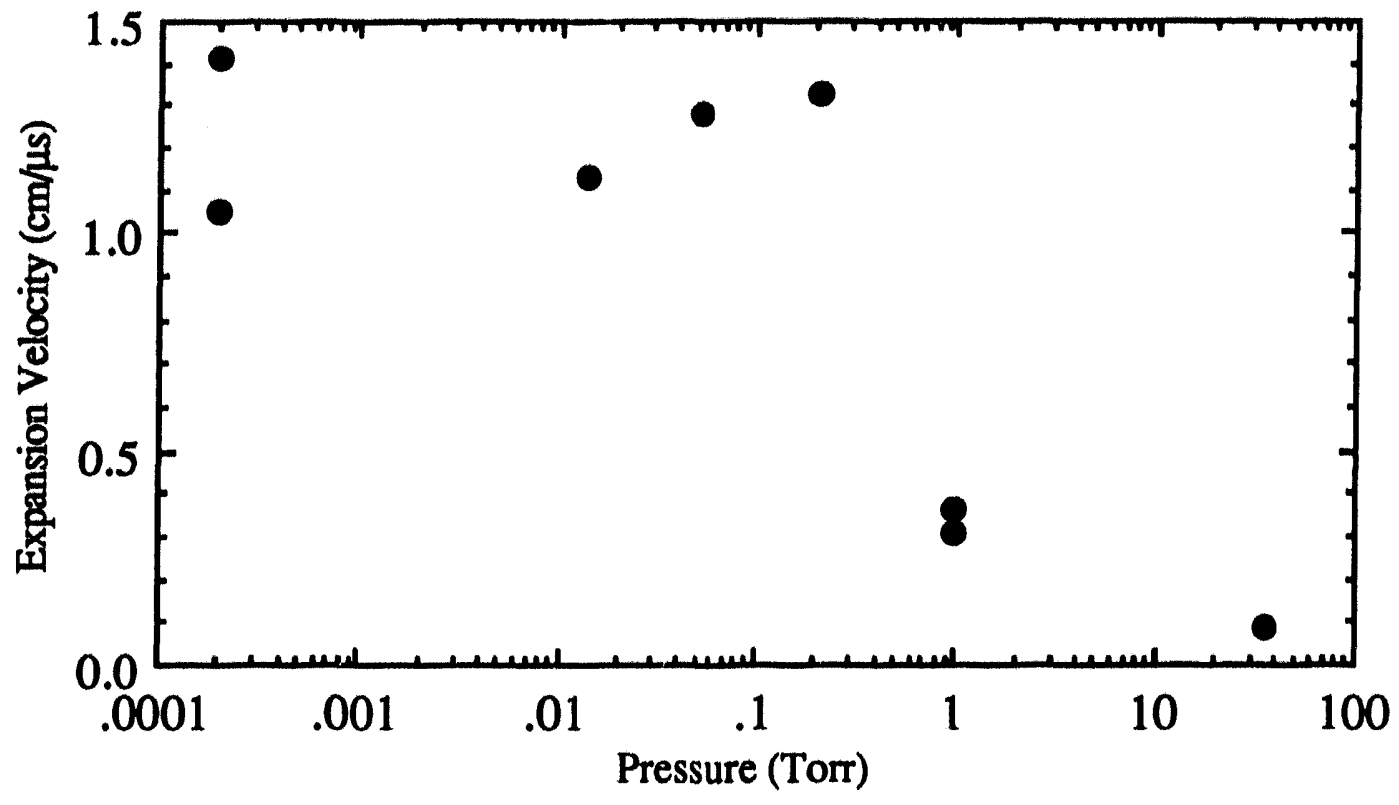

Fig. 4.32 Approximate expansion velocities in vacuum and in argon gas backgrounds. 


\section{CHAPTER 5 CONCLUSIONS}

Resonant Holographic Interferometry (RHI) has been demonstrated to measure the expanding absolute-line-density profiles of aluminum neutral atoms caused by $\mathrm{KrF}$ excimer laser ablation of aluminum targets using a dye laser tuned near the $\mathrm{Al}(\mathrm{I}), 394.401 \mathrm{~nm}$, resonant transition. Dye-Laser-Resonance-Absorption-Photography (DLRAP), which is more sensitive than RHI, has also been utilized to observe the ablation plumes under similar conditions. Plume expansion was studied for $\mathrm{KrF}$ excimer laser ablation $(248 \mathrm{~nm})$ of aluminum $\left(\approx 0.1 \mathrm{~cm}^{2}, 2-6 \mathrm{~J} / \mathrm{cm}^{2}\right)$ in vacuum and in background argon gas pressures of $14 \mathrm{mTorr}, 52 \mathrm{mTorr}, 210 \mathrm{mTorr}$, 1 Torr, and 35 Torr.

The theory developed by Measures (Mea70) to calculate absoluteline-densities from resonant interferometry, which accounted for Doppler shift effects, has been extended to also account for the diagnostic laser bandwidth and the selective absorption of the diagnostic laser by the resonant medium. The results show that for lower line-densities where there is little absorption, Doppler shift effects dominate, causing the observed fringe shift to increase with respect to the value determined from the simplest interferogram interpretation model (Gre64) and as Measures had predicted. For higher line-densities however, selective absorption effects dominate, causing the observed fringe shift to decrease with respect to the simplest interferogram interpretation model. The results also show 
that fringe shift interpretation is not very sensative to the parallel expansion temperature for temperatures in the range $0.1 \mathrm{eV}-1.0 \mathrm{eV}$.

Absolute-line-densities for the aluminum neutral atoms have been measured in the range $4.3 \times 10^{13} \mathrm{~cm}^{-2}$ to $1.0 \times 10^{15} \mathrm{~cm}^{-2}$, which imply aluminum neutral particle densities up to $1 \times 10^{15} \mathrm{~cm}^{-3}$. The total number of aluminum neutral atoms in a plume has been measured to be $\approx 3 \times 10^{14}$. This value corresponds to a suriace etch rate of $1 \mathrm{~nm} / \mathrm{pulse}$, which is close to the etching rate of Kelly and Rothenburg for $2.3 \mathrm{~J} / \mathrm{cm}^{2}$ ablation of aluminum (Kel85). Knowing the reflectivity of aluminum to be $92.5 \%$, the energy absorbed by the target per ablated particle was estimated to be $\approx 466 \mathrm{eV}$, where most of the absorbed energy is presumably dissipated by the aluminum target. This is consistent with the theory that the primary ablation mechanism for metals is thermal ablation (Dre91), where the absorbed laser energy raises the surface temperature causing liquefication and vaporization of the target surface.

The observed ablation plume expansion in vacuum and for pressures $\leq 210 \mathrm{mTorr}$ was consistent with the two-component theory for laser ablation: a fast component of ablated particles, corresponding to ions and charge exchange neutrals accelerated by the ambipolar potential, moved quickly away from the target surface while a slow component of particles lingered near the surface. This expansion model has been discussed and observed by Geohegan using emission photography (Geo92, Geo93) and by Bakos, et al. using TOFPRL (Bak87). Two component expansion is also visible in the PLIF data presented by Cappelli, et al. (Cap90). For most of the holograms in this work, the fast component was at or below the edge of RHI-detectability at the time of separation with the slow component. 
Expansion velocities for the $1 \times 10^{14} \mathrm{~cm}^{-2}$ equicontour line for pressures $\leq 210 \mathrm{~m}$ Torr were in the range $1.05 \mathrm{~cm} / \mu \mathrm{s}$ to $1.41 \mathrm{~cm} / \mu \mathrm{s}$. This is consistent with other recent measurements of the TOF most-probable expansion velocity (Ge089, Wan91, Zhe89) and the leading edge expansion velocity (Dre91, Ven92a, Wal86) of laser ablation expansion in vacuum. Expansion velocities in the range of $0.24 \mathrm{~cm} / \mu \mathrm{s}-0.54 \mathrm{~cm} / \mu \mathrm{s}$ were measured for the center of the plume. The perpendicular expansion velocities measured here correspond to kinetic temperatures in the range 2 $\mathrm{eV}$ to $4 \mathrm{eV}$, which is within the range measured by Dreyfus (Dre86, Dre91) for the ablation of copper under similar conditions.

The expansion of the ablation plume in 1 Torr and 35 Torr argon gas backgrounds was observed to be slower than at pressures less than 210 $\mathrm{mTorr} ; \approx 0.34 \mathrm{~cm} / \mu \mathrm{s}$ was measured for 1 Torr and $\approx 0.084 \mathrm{~cm} / \mu \mathrm{s}$ was measured for 35 Torr. Hydrodynamic effects were also observed, such as mushroom cloud shaped plumes. Sappey and Gamble attribute the mushroom shape for their copper ablation plumes to the exothermic condensation of the atoms to dimers and to the fluid dynamics of one directionally expanding fluid in another (Sap92).

Plume expansion into a 1 Torr argon, RF-plasma was compared with the expansion into a 1 Torr argon gas. It was observed that the ablation plume appeared to expand faster in the plasma and that the total number of aluminum neutrals was lower in the plasma. At this time, it is unknown whether the observed differences are: 1) just statistical variations of the ablation plumes; 2) caused by the RF electric field directly affecting the ablated aluminum ions and electrons at very early times ( $<0.1 \mu \mathrm{s}) ; 3$ ) caused by collisions between the ablation plume and the energetic 
electrons and argon ions during the entire expansion time; or 4) some combination of the above.

Laser ablation deposition of aluminum onto amorphous silicon at room temperature was performed with a target-substrate separation of 1.5 $\mathrm{cm}$. The thin film was observed to have an oval shape, with the larger dimension corresponding to the narrow dimension of the ablation laser spot. This effect has been theoretically modeled and observed in atmospheric air by Miotello, et al. (Mio92), and is confirmation that the expansion of the ablated particles in this experiment is primarily governed by self-collision and self-diffusion mechanisms, especially for expansion into vacuum and lower pressure background gases. 
APPENDICES 


\section{APPENDIX 1 \\ MEASURES' THEORY}

The following is a full derivation of the theory derived from Measures (Mea70). Others who have used or built on this theory include Odenthal, et al. (Ode80), Jellison, et al.(Je181), Kugler, et al. (Kug83), Bone, et al. (Bon86), Craig, et al. (Cra86), and Woisetschluger, et al. (Woi91, Woi92).

The classical model (Mar67) of the interaction of a photon with an atom (or molecule) is described by a sinusoidal electric field, $\underline{E}=E_{0} e^{\text {iox }}$, driving an elastically bound electron whose equation of motion, under a dissipative force proportional to the velocity, is

$$
m \ddot{z}=-m \gamma \dot{z}-m \omega_{0}^{2} z+e E_{0} e^{i \omega x} .
$$

The solution of this equation has the form

$$
z=\frac{e / m}{\omega_{0}^{2}-\omega^{2}+i \gamma \omega} \cdot \mathrm{E}=\frac{\alpha \mathrm{E}}{\mathrm{e}} \text {, }
$$

where $\omega_{0}$ is the resonance frequency of the binding force on the electron, $\gamma$ is the dissipative damping constant, and $\tilde{\alpha}$ is the complex polarizability of the electrically bound electron. The quantum mechanical version of $\tilde{\alpha}$ for an atom in the ith quantum state is (Mar67)

$$
\tilde{\alpha}_{i}=\frac{\left(e^{2} / m\right) f_{i k}}{\omega_{i k}^{2}-\omega^{2}+i \gamma_{k j} \omega},
$$

where $\omega_{i k}$ is the resonance frequency, $f_{i k}$ is the oscillator strength for absorption, and $\gamma_{\mathrm{ki}}$ is the damping constant (to be discussed later). The 
oscillator strength of a transition is related to the Einstein A coefficient for that transition by the relationship (Bershader)

$$
f_{i k}=\frac{4 \pi^{2} \varepsilon_{0} m c^{3}}{\omega_{i k}^{3} e^{2} \hbar} A_{i k} \text {. }
$$

For a photon passing through a collection of atoms, the collection of particles can be treated as a dielectric material. The effective electric field on the atom can then be determined using classical electromagnetic theory (Nay85, Ode80).

Figure A.1 shows the cross section of a dielectric material with dipole moment density, $\overrightarrow{\mathrm{P}}$, in an external electric field, $\overrightarrow{\mathrm{E}}_{\text {ext }}$, both in the $\hat{\mathbf{z}}$ direction. Internally, there is a cavity with atom $\mathrm{A}$ as well as $\mathbf{N}$ other identical atoms. The rest of the material is treated as a continuum. The external field induces a surface charge on the dielectric material, $\sigma_{p}$, and

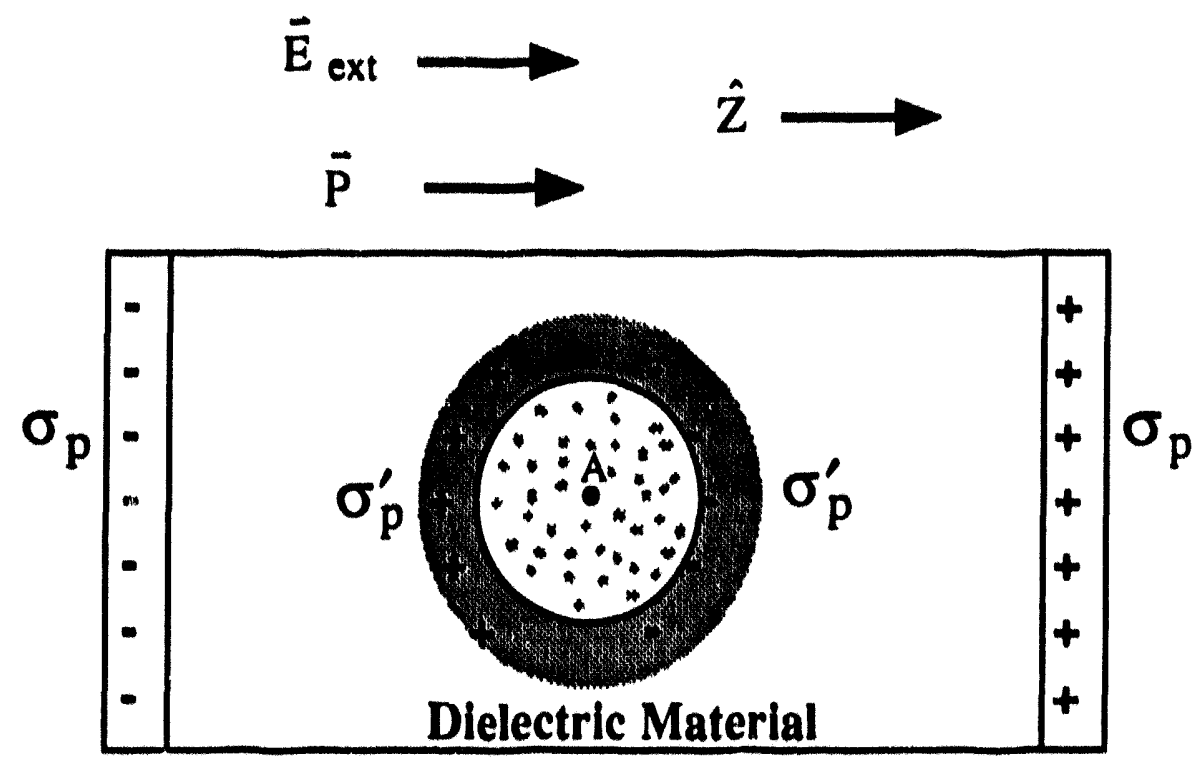

Fig. A.1 Determining the electrical effects on an individual atom of a dielectric material polarized by an external electric field. (Nay85) 
a surface charge around the cavity, $\sigma_{p}^{\prime}$. The electric field as seen by atom $A$ is

$$
E_{n}=E+E_{c a v i t y}+E_{i}
$$

where

$$
\begin{aligned}
& E=(\text { external field })-(\text { dielectric surface charge field })=E_{\text {ext }}-\frac{\vec{P}}{\varepsilon_{0}}(A 1.6) \\
& E_{\text {cavily }}=+\frac{\vec{P}}{3 \varepsilon_{0}},
\end{aligned}
$$

and $E_{i}$ is the field due to all the atoms in the dielectric, which is zero since the dielectric is a fluid for our case. Therefore,

$$
\overrightarrow{\mathrm{E}}_{\mathrm{a}}=\ddot{\mathrm{E}}+\frac{\ddot{\mathrm{P}}}{3 \varepsilon_{0}}
$$

which can also be written

$$
\tilde{\mathrm{P}}=3 \varepsilon_{0}\left(\hat{E}_{\mathrm{a}}-\tilde{E}\right) \text {. }
$$

For linear materials such as fluids, the dipole moment of the material can be written as $\vec{p}=\alpha \vec{E}_{a}$. For $N$ such atoms per unit volume, one obtains

$$
\overrightarrow{\mathrm{P}}=N \overrightarrow{\mathrm{p}}=N \alpha \overrightarrow{\mathrm{E}}_{\mathrm{a}}=N \alpha\left(\overrightarrow{\mathrm{E}}+\frac{\overrightarrow{\mathrm{P}}}{3 \varepsilon_{0}}\right) .
$$

If the dielectric is simple, then $\overrightarrow{\mathrm{P}}=\chi \in \mathbb{E}$ where the electric displacement, $D$, is

$$
\overline{\mathrm{D}}=\varepsilon_{0}(1+\chi) \overrightarrow{\mathrm{E}}=\varepsilon_{0} \mathrm{KE}=\varepsilon \overrightarrow{\mathrm{E}}
$$

which gives us

$$
\tilde{\mathbf{P}}=N \alpha\left(\overline{\mathrm{E}}+\frac{\overrightarrow{\mathrm{P}}}{3 \varepsilon_{0}}\right)=N \alpha\left(\frac{\tilde{\mathrm{P}}}{\chi \varepsilon_{0}}+\frac{\ddot{\mathrm{P}}}{3 \varepsilon_{0}}\right)
$$

and finally leads to the form 


$$
\frac{N \alpha}{3 \varepsilon_{0}}=\frac{\chi}{\chi+3}=\frac{K-1}{K+2}
$$

The index of refraction is defined as

$$
\mathrm{n}=\frac{\mathrm{c}}{\mathrm{v}}=\sqrt{\mathrm{KK}_{\mathrm{m}}}
$$

In most materials, $K_{m} \cong 1$ and so $n^{2} \cong K$. Using the complex index of refraction, where $|\tilde{n}| \cong n \cong 1$ so that $\tilde{n}^{2} \equiv K$, equation $A 1.13$ becomes

$$
\frac{N \alpha}{3 e_{0}} \cong \frac{\tilde{n}^{2}-1}{\tilde{n}^{2}+2} \text {. }
$$

Next, assuming that the dielectric gas in question consists of different species having different polarizabilities $\tilde{\alpha}_{i}$ and densities $N_{i}$, equation A 1.15 becomes

$$
\frac{\tilde{n}^{2}-1}{\tilde{n}^{2}+2}=\frac{1}{3 \varepsilon_{0}} \sum_{i} \tilde{\alpha}_{i} N_{i}=\frac{1}{3 \varepsilon_{0}} \sum_{i} \frac{N_{i} f_{i k}\left(e^{2} / m\right)}{\omega_{i k}^{2}-\omega^{2}+i \gamma_{k i} \omega}
$$

using A1.3.

In truth, this is a simplification of the quantum mechanical events occurring in the atom during an electronic transition. As an electron changes electronic states, the whole electron cloud surrounding the atom changes shape. In turn, the induced polarizability of the atom and therefore the index of refraction change as well. Bershader gives the correct form as (Ber71)

$$
\frac{\tilde{n}_{j}^{2}-1}{\tilde{n}_{j}^{2}+2}=\frac{N_{j} e^{2}}{3 m \varepsilon_{o}}\left\{\sum_{k>j} \frac{f_{j k}}{\omega_{j k}^{2}-\omega^{2}+i \gamma_{k} \omega}-\sum_{k j} \frac{f_{j i}}{\omega_{j i}^{2}-\omega^{2}+i \gamma_{i j} \omega}\right\}
$$

For the derivation here, the second term can be ignored for two reasons. First, this work investigates the index of refraction very near a single, relatively isolated transition. Second, for the $\operatorname{Al}(\mathrm{I}) 32 \mathrm{P}_{1 / 2}-42 \mathrm{~S}_{1 / 2}$, 
$394.4 \mathrm{~nm}$, transition under investigated, there are no electronic transitions to states lower than $32 \mathrm{P}_{1 / 2}$.

So, now assuming one transition of interest, equation A1.16 can be simplified by assuming $\tilde{n}^{2}-1 \cong 2(\tilde{n}-1), \quad \tilde{n}^{2}+2 \cong 3, \quad \omega_{0}=\omega_{i k}, \quad \omega_{0} \cong \omega$, $r_{0}=e^{2} /\left(4 \pi \varepsilon_{0} \mathrm{mc}^{2}\right)=2.818 \times 10^{-13} \mathrm{~cm}$ and

$$
\omega_{0}^{2}-\omega^{2}+i \gamma \omega=\left(\omega_{0}+\omega\right)\left(\omega_{0}-\omega\right)+i \gamma \omega \cong \omega_{0}\left[2\left(\omega_{0}-\omega\right)+i \gamma\right]
$$

and thus giving

$$
\tilde{n}-1=\frac{N_{1} f_{i k} \pi \pi_{0} c^{2}}{\omega_{0}} \frac{\left\{\left(\omega_{0}-\omega\right)-i\left(\frac{\gamma}{2}\right)\right\}}{\left(\omega_{0}-\omega\right)^{2}+\frac{\gamma^{2}}{4}}
$$

Following Marlow (Mar67) and Measures (Mea70), the damping constant $\gamma$ is defined as the sum of the natural, Lorentz, Holtzmark and Stark line-broadenings. The natural broadening term is defined as (Bra92, Ber71)

$$
\gamma_{N}=\sum_{m}\left[A_{i m}+A_{k m}\right]
$$

where the summation is over all of the states $m$ to which an electron can relax from states $\mathrm{i}$ and $\mathrm{k}$ for the transition in question, $\mathrm{i}-\mathrm{k}$. The sum of the Lorentz and Holtzmark broadenings is given as (Bra92)

$\gamma_{L}+\gamma_{H}=\sum_{m} 3.37 \times 10^{10}\left[d_{i}(A)\right]^{2} \sqrt{\frac{1}{M_{i(\text { amu })}}+\frac{1}{M_{m(\text { amu })}}}\left(\frac{P_{m}(\text { atm })}{\sqrt{T_{m}\left({ }^{\circ} k\right)}}\right)$

where the summation is over all gas species $m$ that are present, including the species of interest, $i$, and $d_{i}$ is the effective hard-sphere diameter of the species of interest. The Stark broadening is given as (Bra92) 


$$
\gamma_{S}=2 \pi\left(3 * 10^{18} \frac{\AA}{S}\right) 8.19 * 10^{-19}\left(1-0.7 N_{D}^{-1 / 3}\right)\left(n_{i}^{2}-n_{k}^{2}\right)\left(\frac{z_{p}^{1 / 3}}{Z_{e}}\right)\left(N_{e}\left(\mathrm{~cm}^{-3}\right)\right]^{2 / 3}
$$

where

$$
\mathrm{N}_{\mathrm{D}}=\frac{4 \pi}{3} \mathrm{~N}_{\mathrm{e}} \lambda_{\mathrm{D}}=1.72 * 10^{9} \frac{[\mathrm{T}(\mathrm{eV})]^{3 / 2}}{\left[\mathrm{~N}_{\mathrm{e}}\left(\mathrm{cm}^{-3}\right)\right]^{1 / 2}}
$$

$Z_{p}$ is the charge on the ion, $z_{e}$ is the atomic number, and $n_{i}$ and $n_{k}$ are the principle quantum numbers of the transition $\mathrm{i}-\mathrm{k}$.

Doppler broadening is accounted for assuming that the medium is a neutral gas in Maxwell-Boltzman equilibrium. The Doppler shifted frequency that each atom sees is

$$
\omega^{\prime}=\omega \sqrt{\frac{1+\beta}{1-\beta}}=\omega(1+\beta)^{1 / 2}(1-\beta)^{-1 / 2} \cong \omega\left(1+\beta+\frac{1}{2} \beta^{2}+\ldots\right)
$$

and thus

$$
\omega_{0}^{\prime} \cong \omega_{0}\left(1+\frac{v_{x}}{c}\right)
$$

where the $\hat{\mathrm{x}}$-direction is defined as the direction the diagnostic photons are traveling.

Next, replace $\mathrm{N}$ in equation A1.19 with the Maxwell-Boltzman integral

$$
N_{i}^{\prime}=N_{i}\left(\frac{m}{2 \pi k_{B} T}\right)^{3 / 2} \iint_{-\infty}^{\infty} \int d v_{x} d v_{y} d v_{z} \exp \left(-\frac{m}{2 k_{B} T}\left[v_{x}^{2}+v_{y}^{2}+v_{z}^{2}\right]\right)
$$

(A1.26)

and integrate over $d v_{y}$ and $d v_{z}$ to get the 1-D Maxwellian

$$
N_{i}^{\prime}=N_{i}\left(\frac{m}{2 \pi k_{B} T}\right)^{1 / 2} \int_{-\infty}^{\infty} d v_{x} \exp \left(\frac{-m v_{x}^{2}}{2 k_{B} T}\right)
$$

Dropping the subscript $\mathrm{x}$, equation A1.19 is now written as 


$$
\tilde{n}-1 \cong\left(\frac{\pi r_{0} c^{2} N_{i} f_{i k}}{\omega_{0}}\right)\left(\frac{m}{2 \pi k_{B} T}\right)^{1 / 2} \int_{-\infty}^{\infty} d v \exp \left(\frac{-m v^{2}}{2 k_{B} T}\right) \frac{\left(\omega_{0}\left(1+\frac{v}{c}\right)-\omega\right)-i\left(\frac{\gamma}{2}\right)}{\left(\omega_{0}\left(1+\frac{v}{c}\right)-\omega\right)^{2}+\frac{\gamma^{2}}{4}}
$$

Measures now uses the following simplifications

$$
\beta=\frac{\omega_{0}}{c} \sqrt{\frac{2 k_{B} T}{m}} \quad \alpha=\frac{\gamma_{i k}}{2 \beta} \quad u=\frac{\omega-\omega_{0}}{\beta} \quad y=\frac{v \omega_{0}}{c \beta}
$$

giving

$$
\begin{aligned}
\tilde{n}-1 & \cong\left(\frac{\sqrt{\pi} r_{0} c^{2} N_{i} f_{i k}}{\beta \omega_{0}}\right) \int_{-\infty}^{\infty} d y \frac{(y-u-i \alpha) \exp \left(-y^{2}\right)}{(y-u)^{2}+\alpha^{2}} \\
& \cong\left\{\frac{c}{\omega_{0}}\right\}-i\left(\frac{\pi^{3 / 2} r_{0} c f_{i k}}{\beta}\right)\left(N_{i}\right)\left[\frac{i}{\pi} \int_{-\infty}^{\infty} d y \frac{\exp \left(-y^{2}\right)}{y-[u-i \alpha]}\right] \\
& \cong\left\{\frac{c}{\omega_{0}}\right\}(-i A) N_{i} W(-u+i \alpha)
\end{aligned}
$$

where (Abr72)

$$
W(z)=\exp \left(-z^{2}\right) *(1+\operatorname{erf}\{i z\})
$$

and where $[i \sqrt{\pi} W(z)]$ is the plasma dispersion function.

Using the convention

$$
\underline{E}=\mathrm{E}_{\mathrm{o}} \exp (\mathrm{i} \omega \mathrm{t}-\mathrm{i} \Phi),
$$

for the electric field of the diagnostic photons where

$$
\Phi=\frac{\omega}{c} \int_{0}^{z} \tilde{\mathrm{n}}\left(\omega, \mathrm{z}^{\prime}\right) \mathrm{dz} \mathrm{z}^{\prime}=\frac{\omega \mathrm{z}}{\mathrm{c}}+\frac{\omega}{\mathrm{c}} \int_{0}^{z}\left[\tilde{\mathrm{n}}\left(\omega, \mathrm{z}^{\prime}\right)-1\right] \mathrm{dz} \mathrm{z}^{\prime},
$$

equation $\mathrm{A} 1.30$ becomes

$$
\underline{E}=E_{0} \exp \left[i \omega\left(t-\frac{z}{c}\right)-A \overline{N_{i} L} W(-u+i \alpha)\right]
$$

where $\overline{\mathrm{N}_{\mathrm{i}} \mathrm{L}}$ is the line-integrated-density of the resonant species of the neutral gas in a Maxwell-Boltzmann equilibrium. Finally, this means that 
the measured fringe shiit, $(\Delta s / s)$, of the diagnostic photons is given by the equation

$$
\left(\frac{\Delta s}{s}\right)=\frac{A \overline{N_{i} L}}{2 \pi} \operatorname{Im}[W(-u+i \alpha)]
$$




\section{APPENDIX 2 \\ CALCULATING ROOTS AND FRINGE SHIFTS}

The following is an abbreviated version of the Mathematica program used to calculate the roots and determine the fringe shift for certain values of line-density and dye laser wavelength. The steps are few yet repeated many times with only changes in the input values, thus an abbreviation is given here.

First the roots of equation 2.71 are found by the following program:

$\mathrm{h}\left[\right.$ eps_, dlambda_, $\left.^{\mathrm{nl}}\right]=\operatorname{Exp}\left[-(+518.047 * \text { dlambda }+0.00314 * \mathrm{I} \text { eps })^{\wedge} 2\right]$

$(1+$ Erf $[\mathrm{I}(+518.047 *$ dlambda $+0.00314 * \mathrm{I}-\mathrm{eps})])$ -

((-eps $\left.\left.2.5610^{\wedge} 13 / \mathrm{nl}\right)+\mathrm{I}^{*} 0.5642\right) /\left(+518.047^{*}\right.$ dlambda $+0.00314^{*}$ I-eps $)$

start $=+0.0+0.0 * \mathrm{I}$

$\mathrm{dl}=0.003$

al $=$ FindRoot[h[gam, dl, 1. 10^13] $==0$, (gam,start $)$, MaxIterations $->100]$

itt=First[a1] $/ . \%$

h[First[itt],0.004,1.10^13]

a2 $=$ FindRoot $\left[\mathrm{h}\left[\mathrm{gam}, \mathrm{dl}, 2.10^{\wedge} 13\right]==0\right.$, (gam,First [itt] $)$, MaxIterations $\left.\rightarrow 100\right]$

itt=First[a2] $/ . \%$

$\mathrm{h}[$ First [itt],dl,2. 10^13]

a3 $=$ (etc. ... for the desired values of line-density)

PutAppend[d1,a1,a2,a3, ... ,"roots.h.output.ee2"]

!lroots.h.output.ee2

(this lists them out, so they can be cut and pasted

into a seperate file. The output is alphabetic, not numeric,

so it must be altered manually to obtain a numeric list of

roots)

The function " $\mathrm{h}$ " is defined so that the function is equal to zero and a "start" value for the root search is given along with the dye laser wavelength, "dl", in $\mathrm{nm}$. The root is determined using Newton's Method and each root is checked by inserting the root into the function. If the 
value of "h" using the newly found root is very small (e.g., $\leq 1 \times 10^{-7}$, value checked visually), then the root is valid. This process is repeated for different values of line-density, saved in a file using "PutAppend". The same format is used for other values of dye laser wavelength and then all of the roots are displayed using "!!".

For the solution to equation 2.61 , the definition of " $\mathrm{h}$ " is changed to

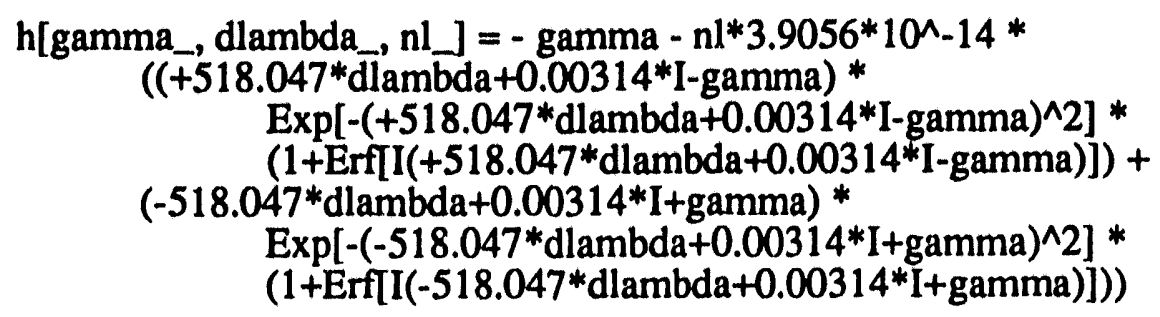

The values $\{518.047\},\{0.00314\}$, and $\left\{3.9056 \times 10^{-14}\right\}$ in the above equations are for the $\mathrm{Al}(\mathrm{I}), 394.401 \mathrm{~nm}$, transition assuming a quasi-equilibrium, Maxwellian temperature of $0.3 \mathrm{eV}$. Note: $\Delta=(-$ $518.047 *$ dlambda) for [dlambda] $=\mathrm{nm}$.

The roots are then reformated (alphabetic to numeric) and fed into the following program to determine fringe shift. The roots to equation 2.61 (gamma) are given here only for completeness.

alpha $=0.00314$

(* if you change alpha, you also have to recalculate all the roots ${ }^{*}$ )

dlambda $=0.003$

delta $=-518.047 *$ dlambda

phi $=+518.047 *$ dlambda + alpha $* I \quad(*$ phi $=-$ delta $+I *$ alpha - gamma/epsilon $*)$

chi $=-518.047 *$ dlambda + alpha*I $\quad(*$ chi $=+$ delta $+I * a l p h a+$ gamma/epsilon $*)$

$w\left[z_{-}\right]=\operatorname{Exp}\left[-\left(z^{\wedge} 2\right)\right]^{*}\left(1+\operatorname{Erf}\left[I^{*} z\right]\right)$

$\operatorname{mr}\left[\mathrm{nl}\right.$, ue_] $=\operatorname{Re}\left[-5.9248 \mathrm{ue}^{\wedge} 2-\mathrm{nl} 2.31410^{\wedge}-13 \mathrm{w}[\mathrm{phi}-\mathrm{ue}]\right]$

iii[nl_, ue $]=\operatorname{Im}\left[-5.9248 \mathrm{ue}^{\wedge} 2-\right.$ nl $2.31410^{\wedge}-13 \mathrm{w}[$ phi-ue]]

(* the roots of eqn. 2.71 at a wavelength $0.003 \mathrm{~nm}$.

"e113" is the value of epsilon for the line-density $1 \times 10^{13} \mathrm{~cm}^{-2} .{ }^{*}$ ) 


$$
\begin{aligned}
& \text { dlambda }=0.003 \\
& \text { e113 }=-0.0488692-0.0547275 * I \\
& \text { e213 }=-0.0899086-0.0991065 * I \\
& \text { e313 }=-0.125766-0.136817 * 1 \\
& \text { e413 }=-0.157883-0.169817 * I \\
& \text { e513 }=-0.187145-0.199285^{*} \mathrm{I} \\
& \text { e613 }=-0.214138-0.225995 * I \\
& \mathrm{e} 713=-0.239277-0.250482 * I \\
& \mathrm{e} 813=-0.262864-0.273132 * \mathrm{I} \\
& \text { e } 913=-0.285131-0.294238 * I \\
& \text { e1 } 14=-0.306256-0.314024 * I \\
& \text { e214 }=-0.476653-0.466151 * I \\
& \text { e } 314=-0.605138-0.573543 * I \\
& \text { e414 }=-0.711273-0.65848 * I \\
& \text { e514 }=-0.803064-0.729634 * I \\
& \text { e } 614=-0.884692-0.791365 * I \\
& \text { e714 }=-0.958649-0.846173 * I \\
& \text { e814 }=-1.02659-0.895716^{*} \mathrm{I} \\
& \text { e914 }=-1.08962-0.941045 * \mathrm{I} \\
& \text { e1 } 15=-1.14858-0.982936 * I \\
& \text { e215=-1.60344 - 1.29332*I } \\
& \text { e } 315=-1.93096-1.50726 * I \\
& \text { e } 415=-2.19515-1.67588 * I \\
& \text { e } 515=-2.42009-1.8174 * \mathrm{I} \\
& \text { e615=-2.6177 - 1.94042*I } \\
& \text { e715 }=-2.79535-2.05027 * I \\
& \text { e815 }=-2.9573-2.14981 * I \\
& \text { e915 }=-3.10665-2.24117 * I
\end{aligned}
$$

(* the roots of eqn. 2.61 at a wavelength $0.003 \mathrm{~nm}$.

"u113" is the value of gamma for the line-density $1 \times 10^{13} \mathrm{~cm}^{-2} .^{*}$ )

dlambda $=0.003$

u113 $=-0.08496619504751728514-0.6031600065665911152 * I$

u213 $=-0.3287336988152596157-0.9348385102125123111 * I$

u313 $=-0.4157594146755698562-1.11150342490483498 * I$

$\mathrm{u} 413=-0.4287888815236202112-1.221998361485870857 * I$

u513 $=-0.4191342770044838794-1.286263394630090693 * I$

u613 $=-0.4067580400263541435-1.324476587177321576 * I$

u713 $=-0.3961177066726843457-1.348931406109431985 * I$

$\mathrm{u} 813=-0.387566875961314686-1.365720590623060003 * \mathrm{I}$

u913 $=-0.3807266757810139003-1.37790624074367299 * I$

u114=-0.3751930324789996254 - $1.387137341517644663 * I$ u2 $14=-0.3503887742179140818-1.423691049793081242 * I$ u314 $=-0.3423466874362853795-1.434323734296974958 * I$ u414=-0.3383895101318873075 - $1.4393925770574698 * I$ u514=-0.3360372432247489145 - $1.442359651139959834 * I$ u614=-0.3344785402432459137 - 1.444307854152442616*I u714=-0.3333698990835633334 - $1.445685143136329258 * I$ $\mathrm{u} 814=-0.3325410256628195829-1.446710421720261326 * \mathrm{I}$ u914 $=-0.3318979025210007285-1.447503358243030367 * I$ 
u115 $=-0.3313843894490563171-1.448134896706126034 * I$ u215 $=-0.3290845085317007846-1.450946390829147029 * I$ u315 $=-0.3283218879021801245-1.451872668165625699 * \mathrm{I}$ u415 $=-0.3279413331011181412-1.452333798993440078 * \mathrm{I}$ u $515=-0.3277132427422407516-1.452609838765402512 * I$ u615 $=-0.3275612836900032736-1.452793600074777329 * I$ u715 $=-0.3274527911122523638-1.452924728549267829 * I$ $\mathrm{u} 815=-0.3273714488208655585-1.453023004140050014 * \mathrm{I}$ u915 $=-0.3273081986851128786-1.453099398824839781 * I$

fsa $=$ iii $\left[1.10^{\wedge} 13\right.$, el13] *0.1592

(* Calculating Fringe Shift (FS)

*)

$\mathrm{fsb}=\mathrm{iii}\left[2.10^{\wedge} 13, \mathrm{e} 213\right] * 0.1592$

$\mathrm{fsc}=\mathrm{iii}\left[3.10^{\wedge} 13, \mathrm{e} 313\right] * 0.1592$

fsd $=$ iii $\left[4.10^{\wedge} 13\right.$, e 413$] * 0.1592$

fse $=\mathrm{iii}\left[5.10^{\wedge} 13, \mathrm{e} 513\right] * 0.1592$

$\mathrm{fsf}=\mathrm{iii}\left[6.10^{\wedge} 13, \mathrm{e} 613\right] * 0.1592$

$\mathrm{fsg}=\mathrm{iii}\left[7.10^{\wedge} 13, \mathrm{e} 713\right] * 0.1592$

$\mathrm{fsh}=\mathrm{iii}\left[8.10^{\wedge} 13, \mathrm{e} 813\right] * 0.1592$

fsi $=\mathrm{iii}\left[9.10^{\wedge} 13, \mathrm{e} 913\right] * 0.1592$

fsj=iii $\left[1.10^{\wedge} 14\right.$, el14] $* 0.1592$

$\mathrm{fsk}=\mathrm{iii}\left[2.10^{\wedge} 14, \mathrm{e} 214\right] * 0.1592$

fsl=iii[3. $\left.10^{\wedge} 14, \mathrm{e} 314\right] * 0.1592$

fsm=iii[4. 10^14, e414] *0.1592

fsn $=$ iii $\left[5.10^{\wedge} 14, \text { e } 514\right]^{*} 0.1592$

fso=iii[6. $10^{\wedge} 14$, e614] $* 0.1592$

$\mathrm{fsp}=\mathrm{iii}\left[7.10^{\wedge} 14, \mathrm{e} 714\right] * 0.1592$

fsq $=$ iii $\left[8.10^{\wedge} 14\right.$, e814] $* 0.1592$

fsr $=$ iii $\left[9.10^{\wedge} 14\right.$, e914] $* 0.1592$

fss $=$ iii $\left[1.10^{\wedge} 15\right.$, el15] $* 0.1592$

fst $=$ iii $\left[2.10^{\wedge} 15, \mathrm{e} 215\right] * 0.1592$

$\mathrm{fsu}=\mathrm{iii}\left[3.10^{\wedge} 15, \mathrm{e} 315\right] * 0.1592$

fsv $=$ iii $\left[4.10^{\wedge} 15\right.$, e 415$] * 0.1592$

fsx $=$ iii $\left[5.10^{\wedge} 15, \mathrm{e} 515\right] * 0.1592$

fsy $=$ iii $\left[6.10^{\wedge} 15\right.$, e615] $* 0.1592$

fsz=iii[7. 10^15, e715]*0.1592

fszaa $=$ iii $\left[8.10^{\wedge} 15\right.$, e815] $* 0.1592$

fszbb $=$ iii $\left[9.10^{\wedge} 15, e 915\right] * 0.1592$

PutAppend[dlambda, aaee,e113,e213,e313,e413,e513,e613,e713,e813,e913, e1 14,e214,e314,e414,e514,e614,e714,e814,e914, e1 15,e215,e315,e415,e515,e615,e715,e815,e915, aauu, u113,u213,u313,u413,u513,u613,u713,u813,u913, $\mathrm{u} 114, \mathrm{u} 214, \mathrm{u} 314, \mathrm{u} 414, \mathrm{u} 514, \mathrm{u} 614, \mathrm{u} 714, \mathrm{u} 814, \mathrm{u} 914$, $\mathrm{u} 115, \mathrm{u} 215, \mathrm{u} 315, \mathrm{u} 415, \mathrm{u} 515, \mathrm{u} 615, \mathrm{u} 715, \mathrm{u} 815, \mathrm{u} 915$, aafs,fsa,fsb,fsc, fsd, fse, fsf, fsg, fsh, fsi,fsj,fsk,fsl,fsm,fsn,fso,fsp, fsq, fsr, fss,fst, fsu,fsv, fsx,fsy,fsz,fszaa,fszbb, "roots.h.output.f003"]

!!roots.h.output.f003 
(* This is the Sept. 1993 streamline model of the calculation of the fringe shift. Just enter the temperature and the wavelength in at the top, and the program will give you the fringe shift for the preselected line-densities. Next, linearly interpolate to find the line-density for a given fringe shift.

Roger Alan Lindley

(* the notation "e113" is to be read as the epsilon root for the line density $1 \times 10^{\wedge} 13 \mathrm{~cm}-2{ }^{*}$ )

temp $=3.0 \quad\left({ }^{*}\right.$ enter in the temperature in $\left.[\mathrm{eV}]^{*}\right)$

$\mathrm{dl}=0.006 \quad$ (* enter in the wavelength in $[\mathrm{nm}]{ }^{*}$ )

alpha $=0.00314^{*}(0.3 / \text { temp })^{\wedge} 0.5$

dddd $=518.047 *(0.3 / \text { temp })^{\wedge} 0.5$

kkkk=2.56 $10^{\wedge} 13^{*}(\text { temp } / 0.3)^{\wedge} 1.5$

cccc $=5.9248^{*}(\text { temp } / 0.3)^{\wedge} 1.0$

aaaa $=2.31410^{\wedge}-13^{*}(0.3 / \text { temp })^{\wedge} 0.5$

$w\left[z_{-}\right]=\operatorname{Exp}\left[-\left(z^{\wedge} 2\right)\right] *\left(1+\operatorname{Erf}\left[I^{*} z^{2}\right]\right)$

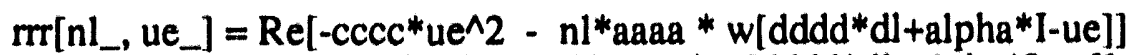

iii $\left[n_{-}, u e_{-}\right]=\operatorname{Im}\left[-\operatorname{cccc}^{*} u e^{\wedge} 2-n l^{*}\right.$ aaaa $\left.* w\left[d d d d * d l+a l p h a^{*} I-u e\right]\right]$

(* MEASURES' ANSWER for line-density per fringe shift: *) nldss $=(2 * 3.14159 /$ aaaa $) /\left(\operatorname{Im}\left[w\left[+\right.\right.\right.$ dddd $\left.\left.\left.^{*} \mathrm{dl}+\mathrm{alpha}{ }^{*} \mathrm{I}\right]\right]\right)$

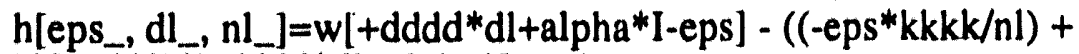
I*0.5642)/(+dddd*dl+alpha*I-eps)

a1 $=$ FindRoot[h[eps, dl, 1. 10 13] $==0,\{$ eps, 0.0$\}$, MaxIterations $\rightarrow$ 100] itt=First[a1] $/ . \%$

e113=First[itt]

$\mathrm{h}\left[\mathrm{e} 113, \mathrm{dl}, 1.10^{\wedge} 13\right]$

a2 $=$ FindRoot[h[eps, dl, 2. 10^13] $==0,\{$ eps,el13\}, MaxIterations $\rightarrow$ 100]

$\mathrm{itt}=$ First[a2] $1 . \%$

e213=First[itt $]$

$\mathrm{h}\left[\mathrm{e} 213, \mathrm{dl}, 2.10^{\wedge} 13\right]$

$\mathrm{a} 3=$ FindRoot $\left[\mathrm{h}\left[\mathrm{eps}, \mathrm{dl}, 3.10^{\wedge} 13\right]==0,(\mathrm{eps}, \mathrm{e} 213\}\right.$, MaxIterations $\left.->100\right]$

itt=First[a3] $/ . \%$

e313=First[itt]

$\mathrm{h}\left[\mathrm{e} 313, \mathrm{dl}, 3.10^{\wedge} 13\right]$

(* the program is abbreviated here, continue the above for all of the desires values of line density ${ }^{*}$ )

fsa $=$ iii $\left[1.10^{\wedge} 13\right.$, e 113$] * 0.1592$

$\mathrm{fsb}=\mathrm{iii}\left[2.10^{\wedge} 13, \mathrm{e} 213\right] * 0.1592$

$\mathrm{fsc}=\mathrm{iii}\left[3.10^{\wedge} 13, \mathrm{e} 313\right] * 0.1592$

fsd=iii[4. $10^{\wedge} 13$, e 413$] * 0.1592$ 
fse $=$ iii $\left[5.10^{\wedge} 13\right.$, e513] $* 0.1592$

$\mathrm{fsf}=\mathrm{iii}\left[6.10^{\wedge} 13, \mathrm{e} 613\right] * 0.1592$

fs $g=$ iii $\left[7.10^{\wedge} 13, e 713\right] * 0.1592$

$\mathrm{fsh}=\mathrm{iii}\left[8.10^{\wedge} 13, \mathrm{e} 813\right] * 0.1592$

fsi=iii $\left[9.10^{\wedge} 13, e 913\right] * 0.1592$

fsj $=$ iili $\left[1.10^{\wedge} 14\right.$, e1 14] * 0.1592

$\mathrm{fsk}=\mathrm{iii}\left[2.10^{\wedge} 14, \mathrm{e} 214\right] * 0.1592$

fsl $=\mathrm{iii}\left[3.10^{\wedge} 14, \mathrm{e} 314\right] * 0.1592$

fsm=ili $\left[4.10^{\wedge} 14, e 414\right] * 0.1592$

fsn=iii[5. 10^14, e514] *0.1592

fso=iii $\left[6.10^{\wedge} 14, e 614\right] * 0.1592$

$\mathrm{fsp}=\mathrm{iii}\left[7.10^{\wedge} 14, \mathrm{e} 714\right] * 0.1592$

fsq $=$ iii $\left[8.10^{\wedge} 14, e 814\right] * 0.1592$

fsr $=$ iii $\left[9.10^{\wedge} 14\right.$, e 914$] * 0.1592$

fss $=\mathrm{iii}\left[1.10^{\wedge} 15, \mathrm{el} 15\right] * 0.1592$

fst $=\mathrm{iii}\left[2.10^{\wedge} 15, \mathrm{e} 215\right] * 0.1592$

fsu $=$ iii [3. 10^15, e315]*0.1592

$\mathrm{fsv}=\mathrm{iii}\left[4.10^{\wedge} 15, \mathrm{e} 415\right] * 0.1592$

fs $x=$ iii $\left[5,10^{\wedge} 15\right.$, e 515$] * 0.1592$

fsy $=$ iii $\left[6.10^{\wedge} 15, e 615\right] * 0.1592$

fsz $=$ iii $\left[7.10^{\wedge} 15\right.$, e 715$] * 0.1592$

fszaa=iii $\left[8.10^{\wedge} 15\right.$, e815] * 0.1592

fszbb=iii[9. $10^{\wedge} 15$, e915] * 0.1592

PutAppend[dl,temp,

aaee,e113,e213,e313,e413,e513,e613,e713,e813,e913,e114,e214,e314,e414,e514,e61 $4, \mathrm{e} 714, \mathrm{e} 814, \mathrm{e} 914, \mathrm{e} 115, \mathrm{e} 215, \mathrm{e} 315, \mathrm{e} 415, \mathrm{e} 515, \mathrm{e} 615, \mathrm{e} 715, \mathrm{e} 815, \mathrm{e} 915$,

aafs, fsa,fsb,fsc,fsd,fse, fsf, fsg, fsh,fsi,fsj, fsk,fsl,fsm,fsn,fso,fsp,fsq, fsr,fss, fst,fsu,fsv ,fsx,fsy,fsz,fszaa,fszbb,

"roots.h.output.final.1"]

Ilroots.h.output.final.1 


\section{APPENDIX 3 \\ FRINGE SHIFT INTERPRETATION GRAPHS}

The following graphs represent the fringe shift interpretations for the holograms presented in Chapter 4 as calculated by Griem (eqn. 2.9) assuming an infinitesimal laser bandwidth and no Doppler shift effects (cold ablation plume), by Measures (eqn. 2.86) accounting for the Doppler shift effects and assuming an infinitesimal laser bandwidth, by Dreiden (eqn. 2.28) accounting for a finite laser bandwidth but assuming no Doppler shift effects and no selective absorption effects, and by this work (eqn. 2.85) accounting for Doppler shift effects, finite laser bandwidth, and selective absorption effects. 


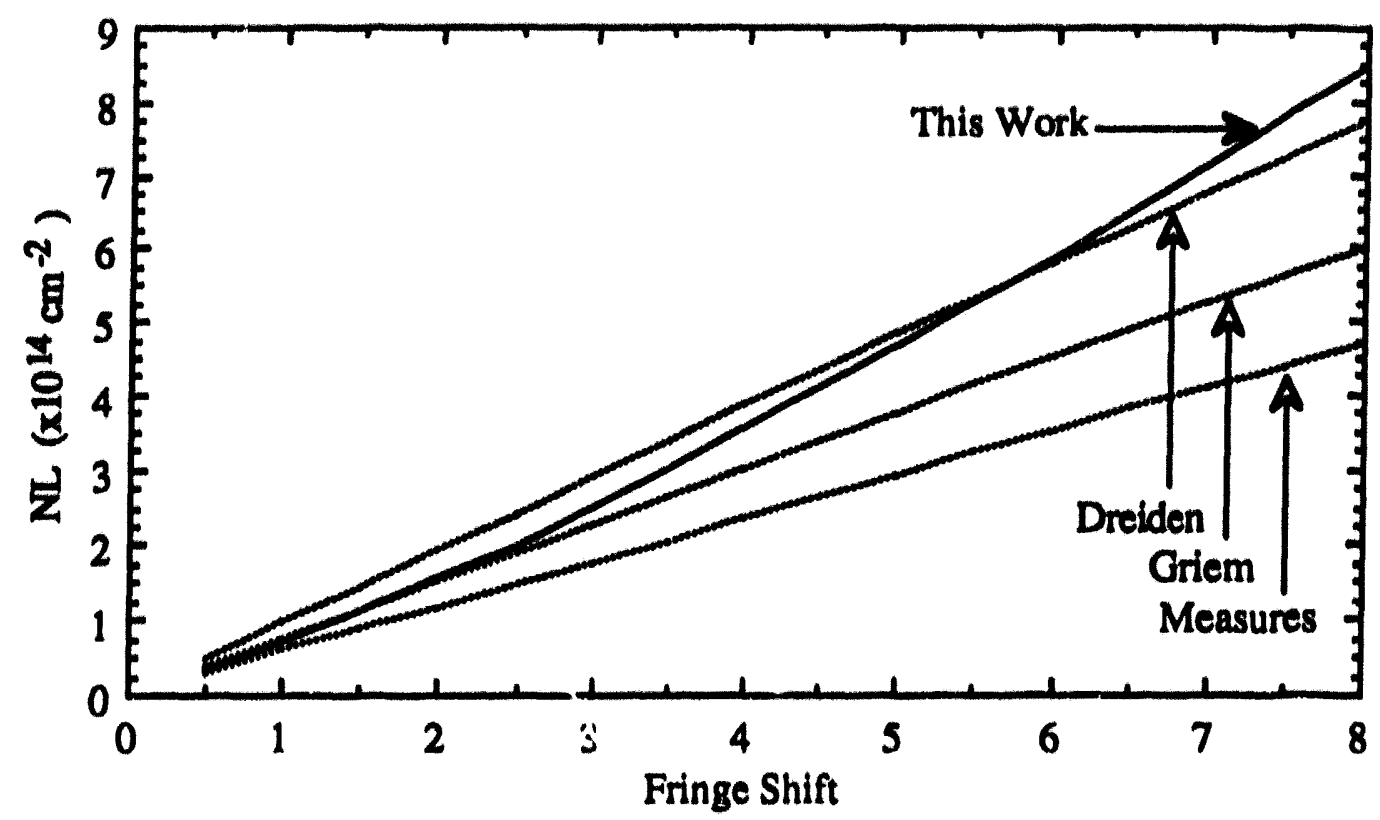

Fig. A3.1 Fringe shift interpretation at $0.003 \mathrm{~nm}$ for the $\mathrm{Al}(\mathrm{I})$ $394.401 \mathrm{~nm}$ line assuming a plume temperature of $0.3 \mathrm{eV}$.

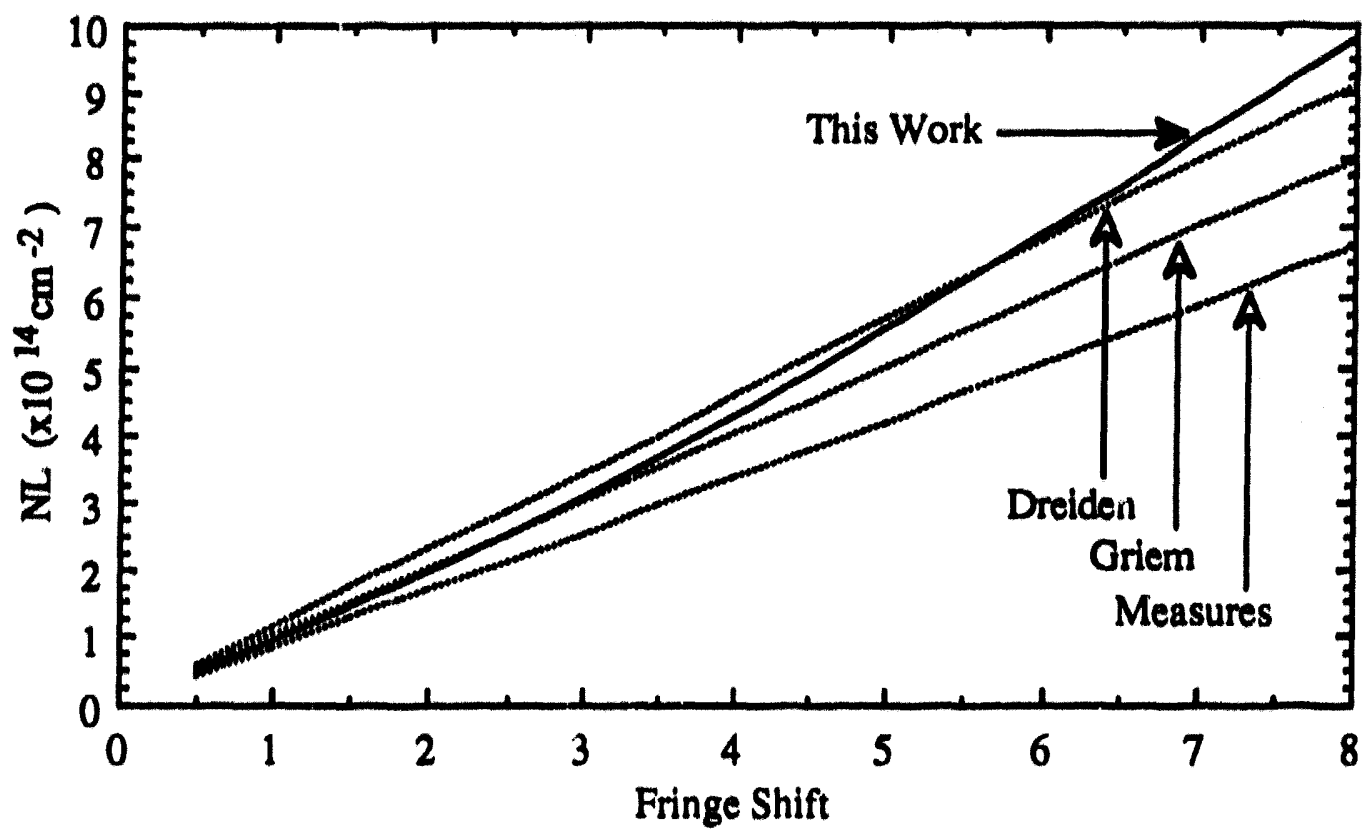

Fig. A3.2 Fringe shift interpretation at $0.004 \mathrm{~nm}$ for the $\mathrm{Al}(\mathrm{I})$ $394.401 \mathrm{~nm}$ line assuming a plume temperature of $0.3 \mathrm{eV}$. 


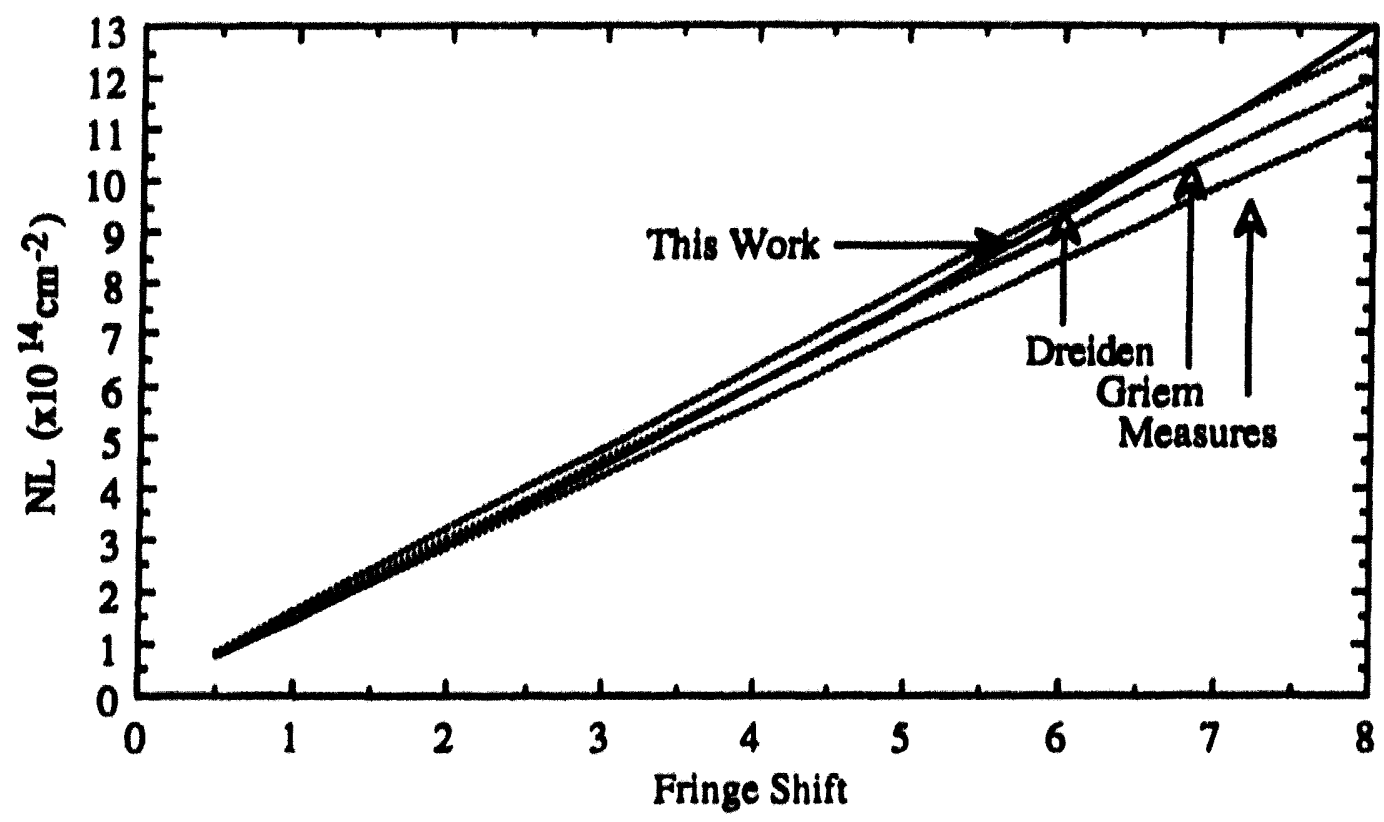

Fig. A3.3 Fringe shift interpretation at $0.006 \mathrm{~nm}$ for the $\mathrm{Al}(\mathrm{I})$ $394.401 \mathrm{~nm}$ line assuming a plume temperature of $0.3 \mathrm{eV}$.

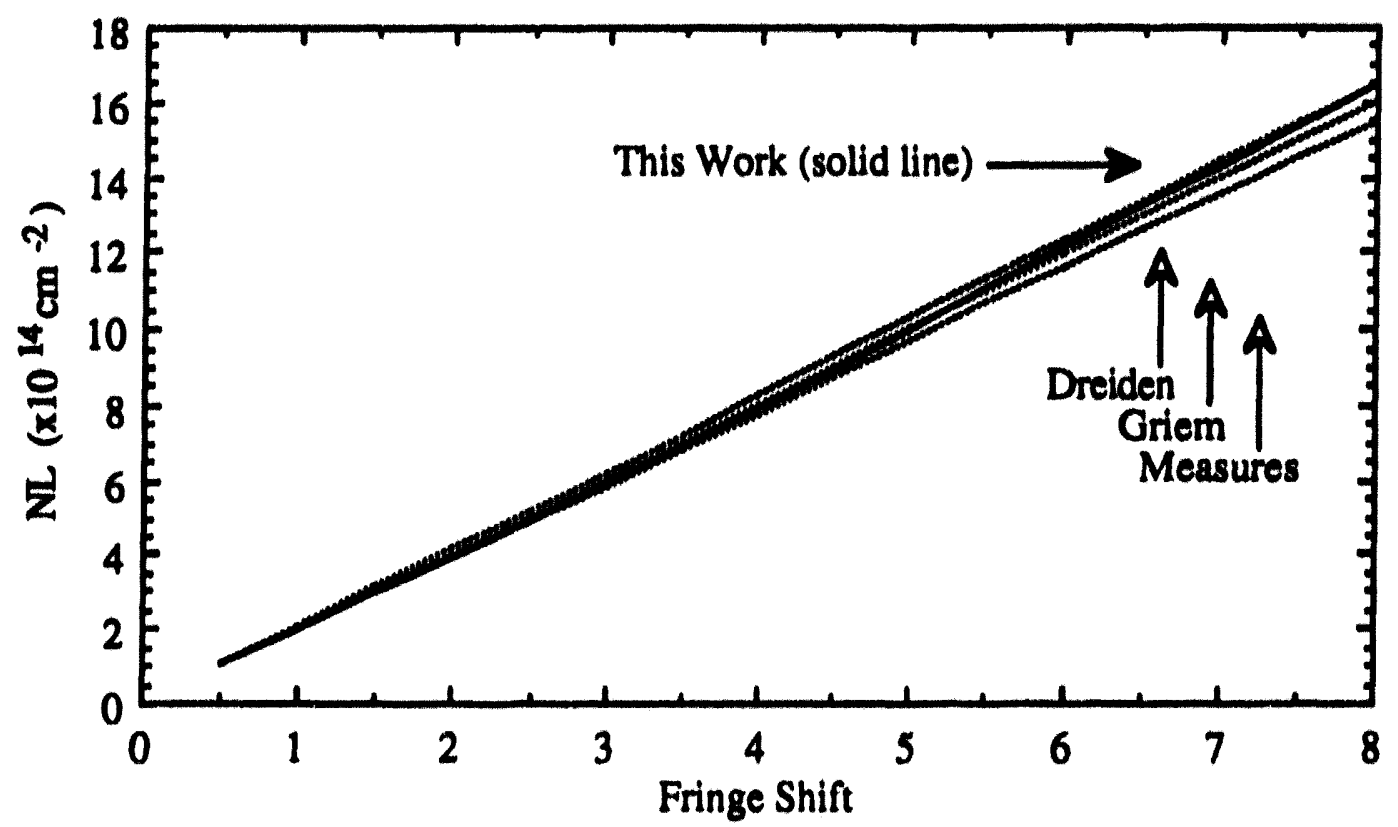

Fig. A3.4 Fringe shift interpretation at $0.008 \mathrm{~nm}$ for the $\mathrm{Al}(\mathrm{I})$ $394.401 \mathrm{~nm}$ line assuming a plume temperature of $0.3 \mathrm{eV}$. 


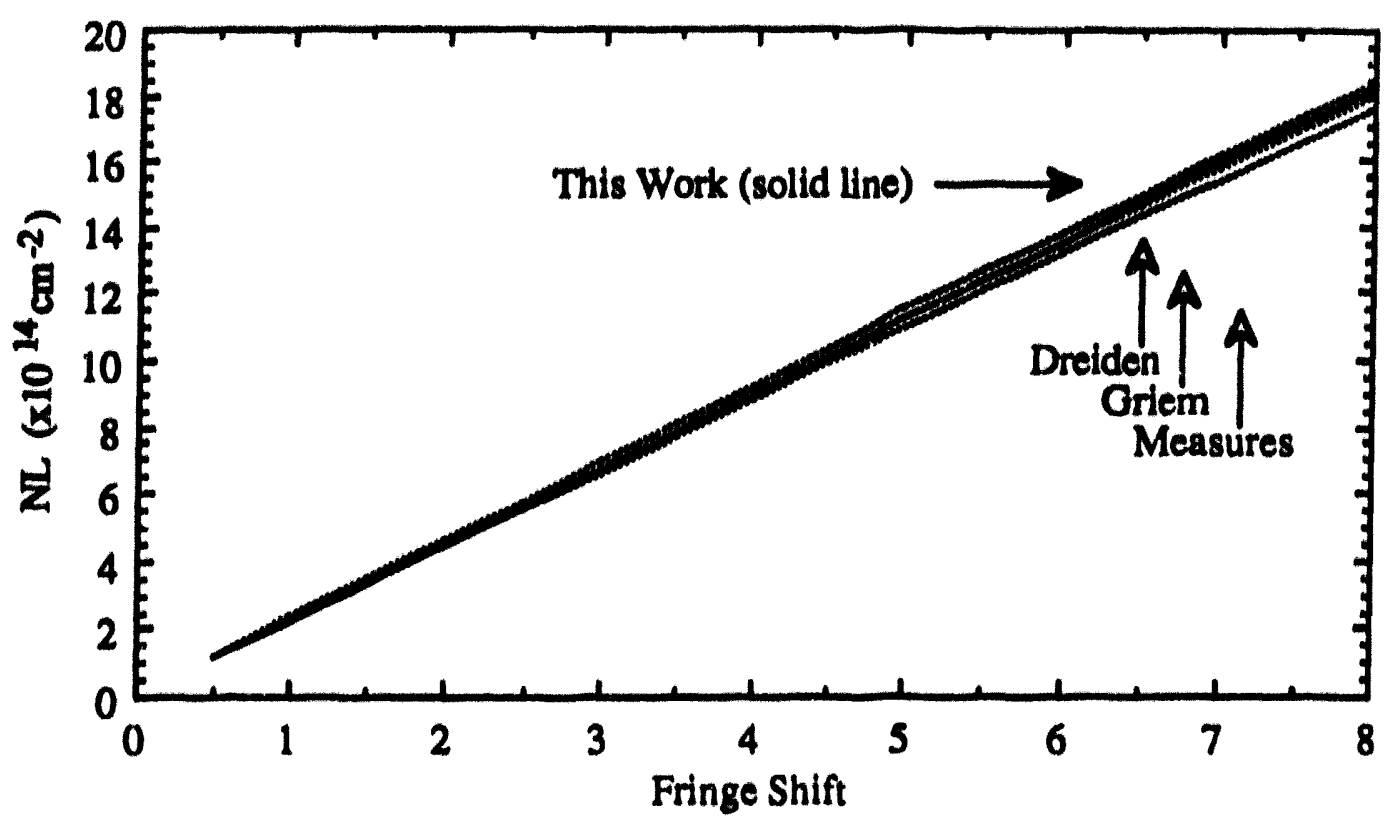

Fig. A3.5 Fringe shift interpretation at $0.009 \mathrm{~nm}$ for the $\mathrm{Al}(\mathrm{I})$ $394.401 \mathrm{~nm}$ line assuming a plume temperature of $0.3 \mathrm{eV}$.

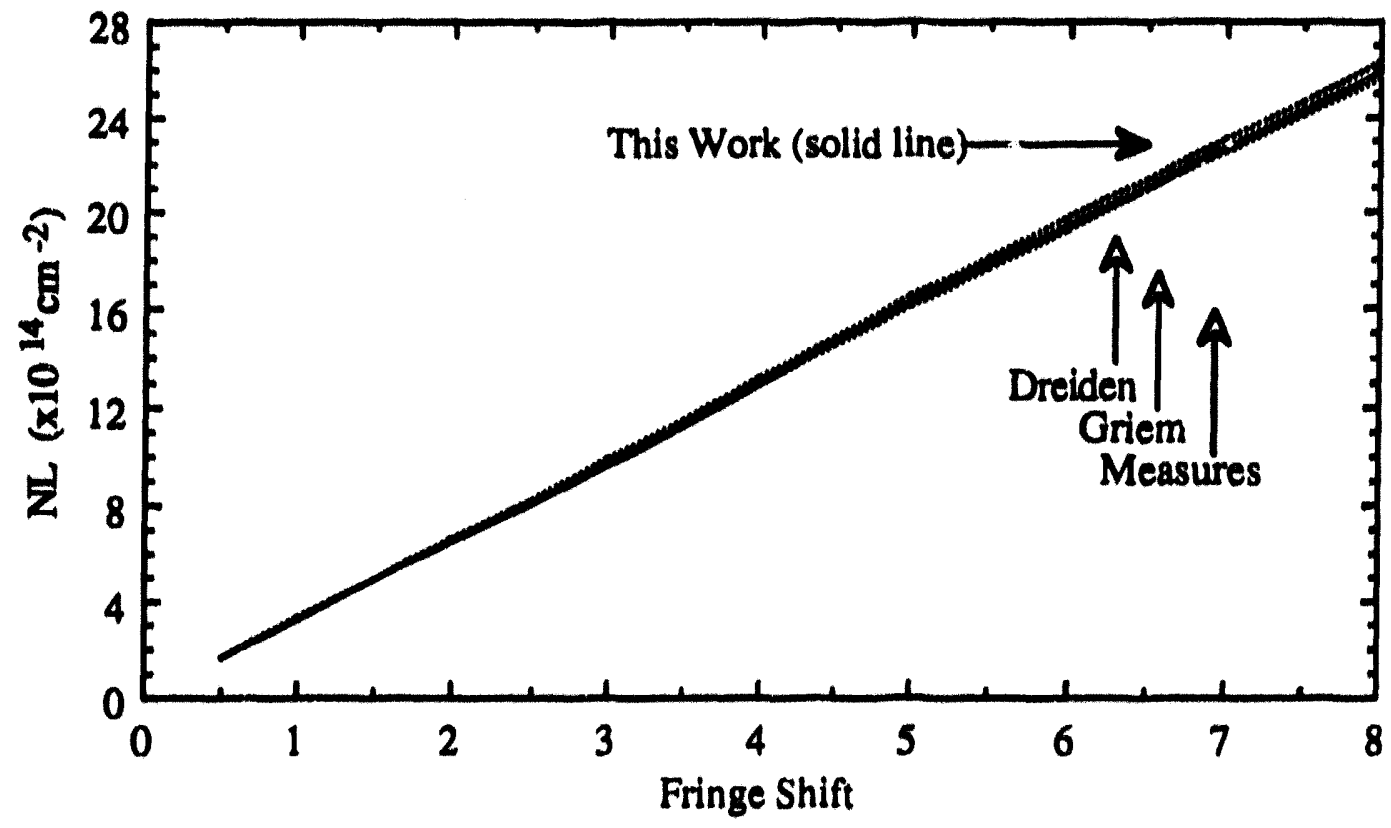

Fig. A3.6 Fringe shift interpretation at $0.013 \mathrm{~nm}$ for the $\mathrm{Al}(\mathrm{I})$ $394.401 \mathrm{~nm}$ line assuming a plume temperature of $0.3 \mathrm{eV}$. 


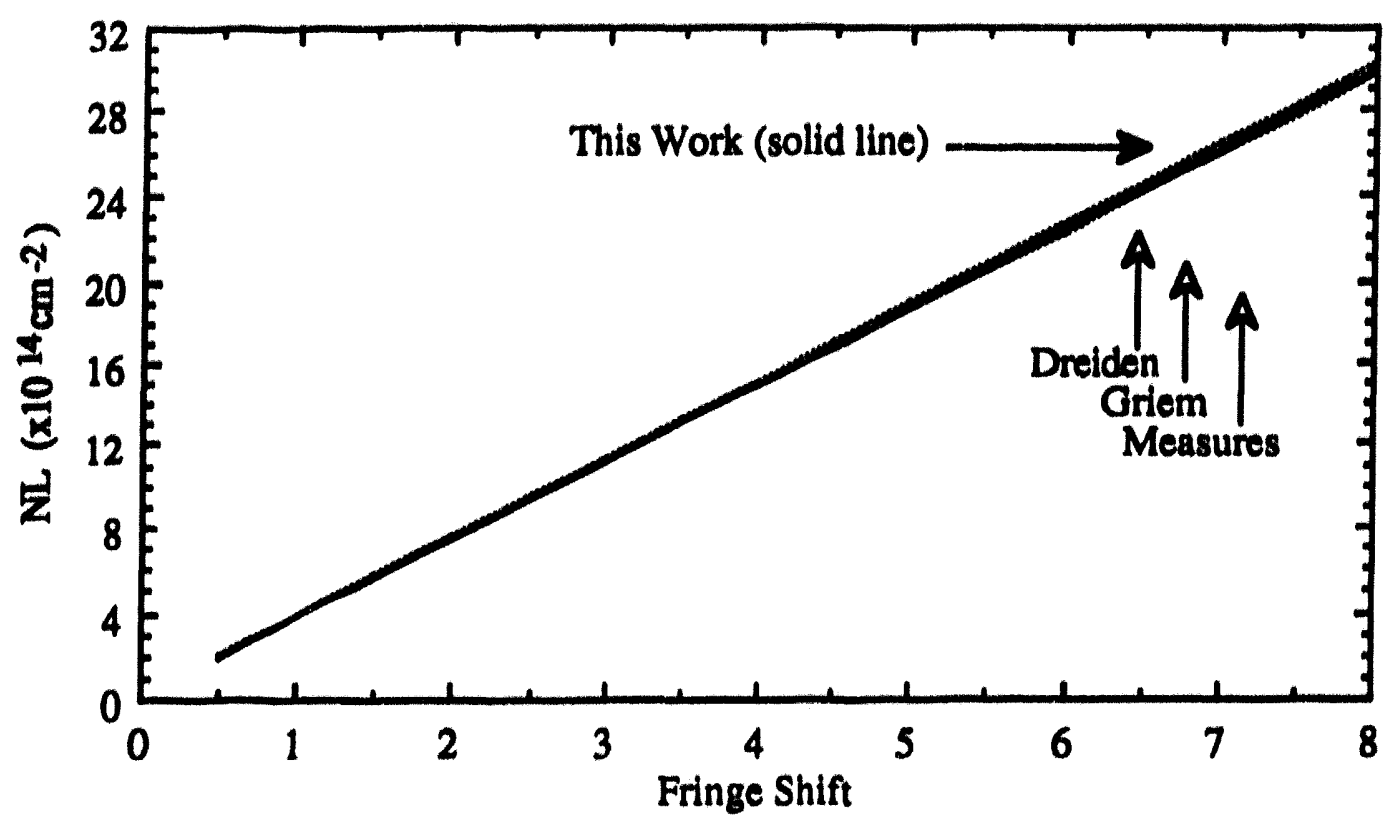

Fig. A3.7 Fringe shift interpretation at $0.015 \mathrm{~nm}$ for the $\mathrm{Al}(\mathrm{I})$ $394.401 \mathrm{~nm}$ line assuming a plume temperature of $0.3 \mathrm{eV}$.

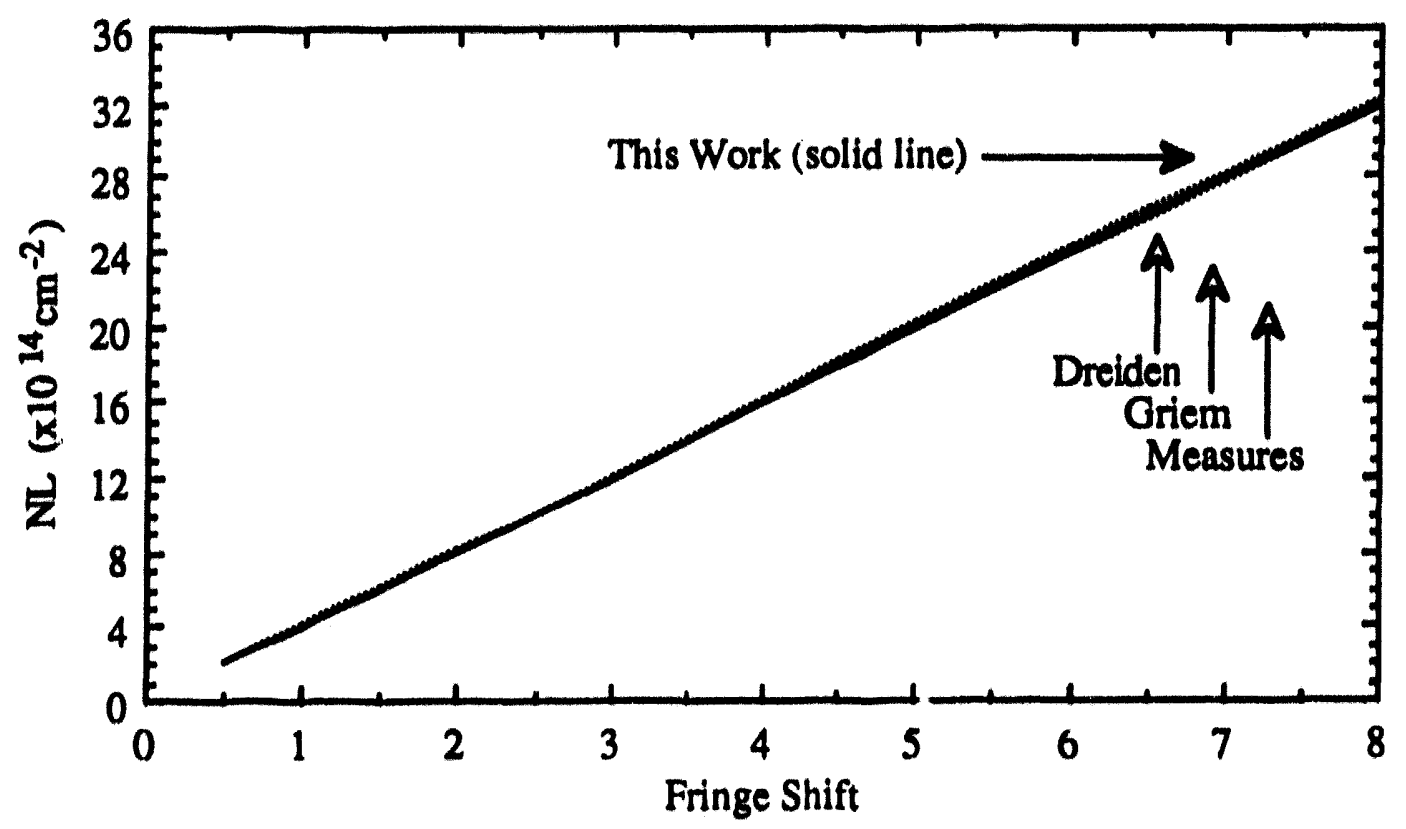

Fig. A3.8 Fringe shift interpretation at $0.016 \mathrm{~nm}$ for the $\mathrm{Al}(\mathrm{I})$ $394.401 \mathrm{~nm}$ line assuming a plume temperature of $0.3 \mathrm{eV}$. 


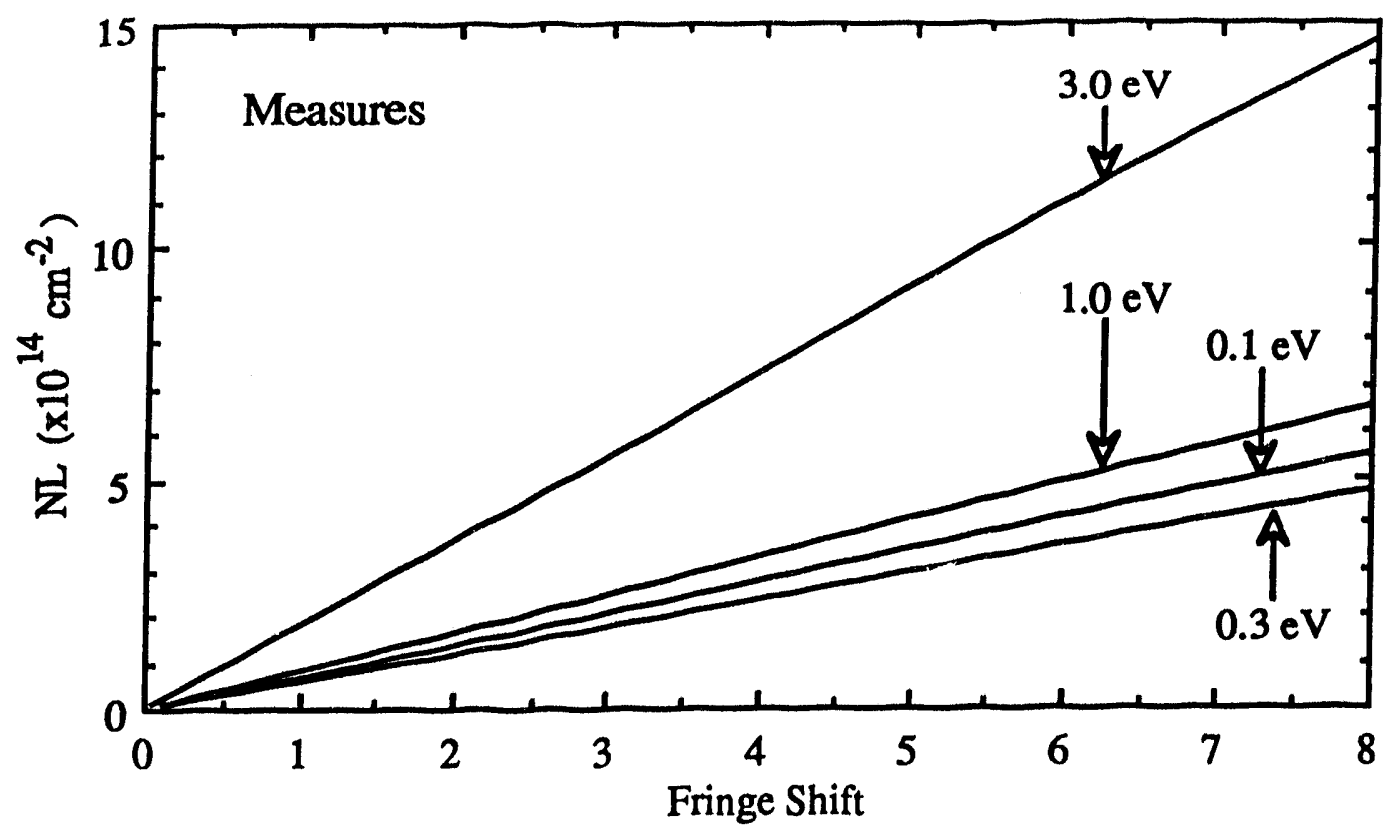

Fig. A3.9 Measures' calculation at $0.003 \mathrm{~nm}$ for several ablation plume temperatures.

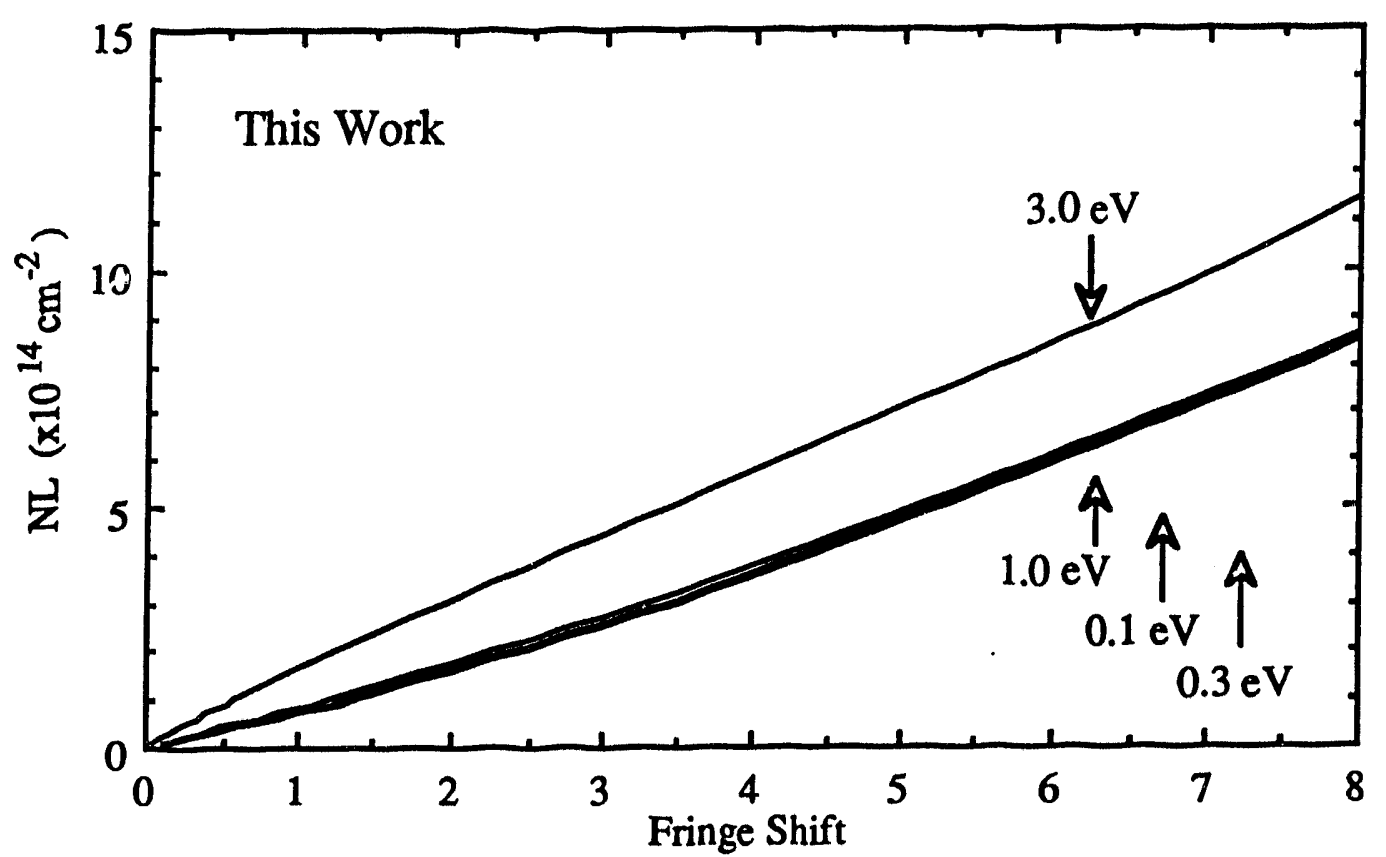

Fig. A3.10 This work's calculation at $0.003 \mathrm{~nm}$ for several ablation plume temperatures. 


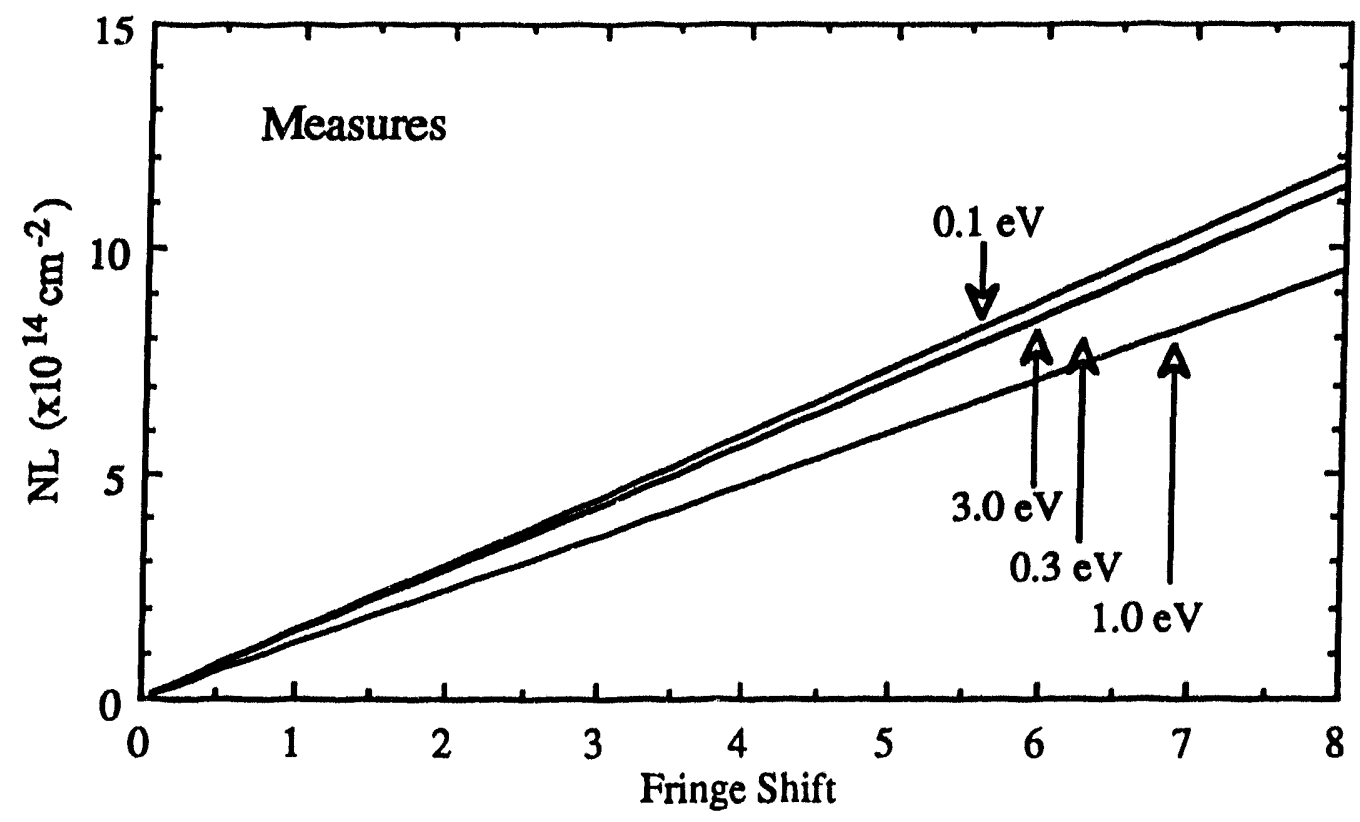

Fig. A3.11 Measures' calculation at $0.006 \mathrm{~nm}$ for several ablation temperatures.

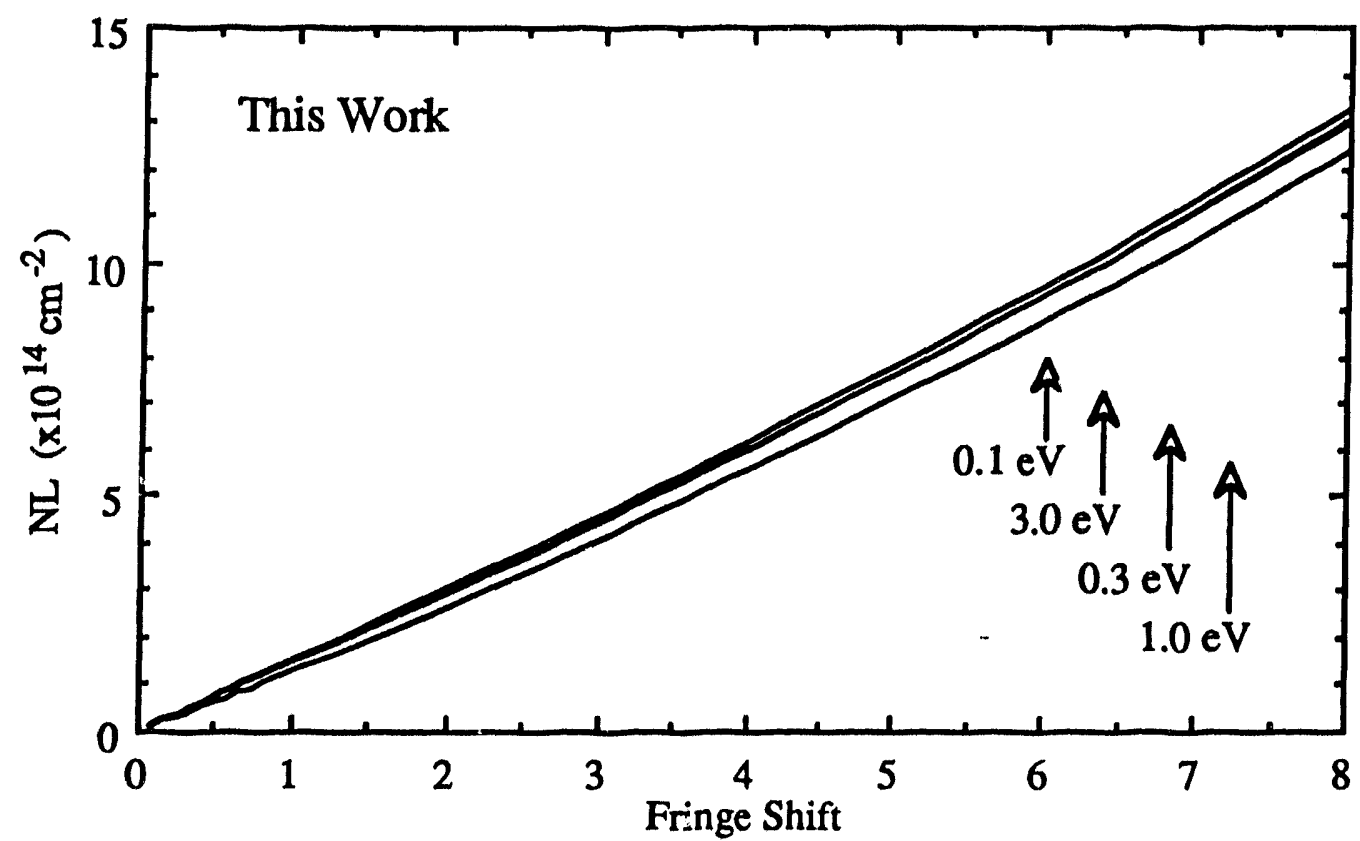

Fig. A3.12 This work's calculation at $0.006 \mathrm{~nm}$ for several ablation plume temperatures. 


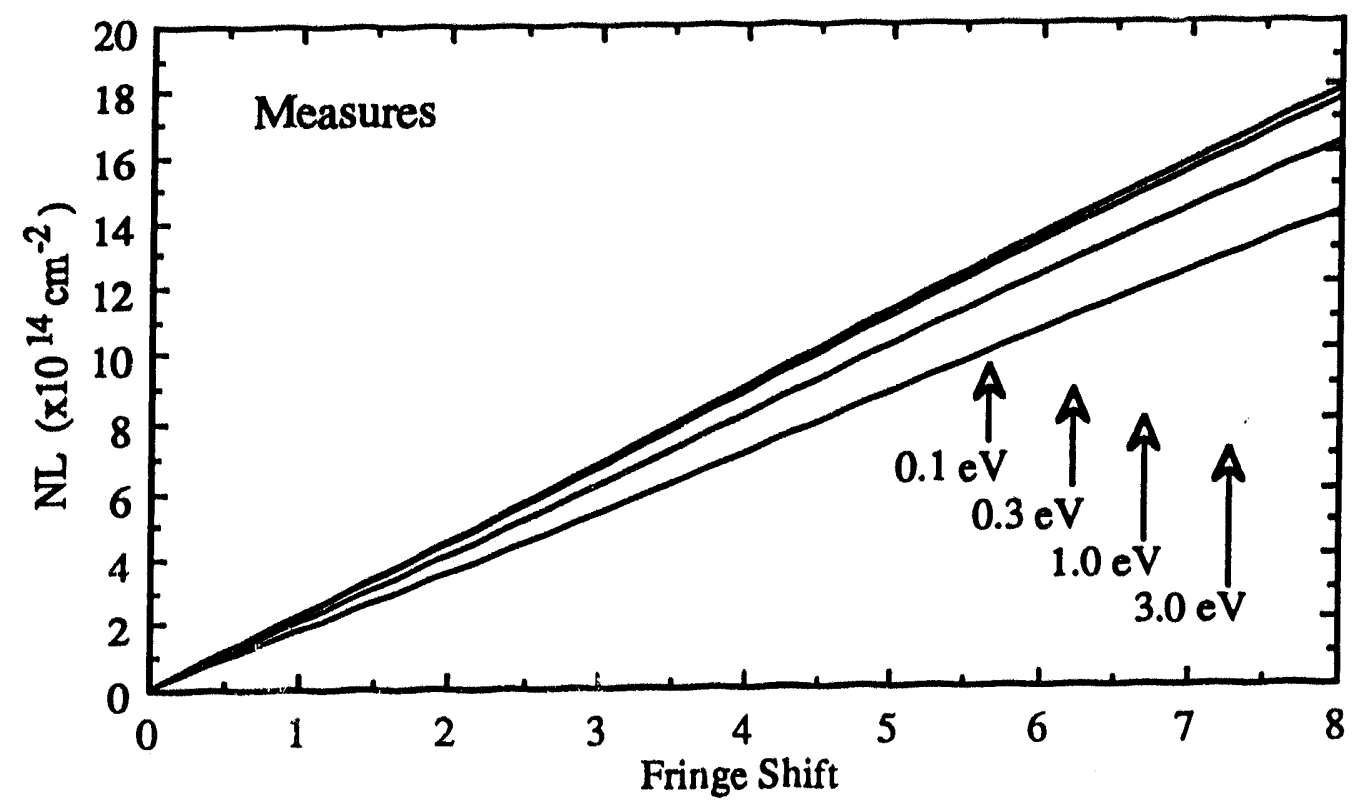

Fig. A3.13 Measures' calculation at $0.009 \mathrm{~nm}$ for several ablation plume temperatures.

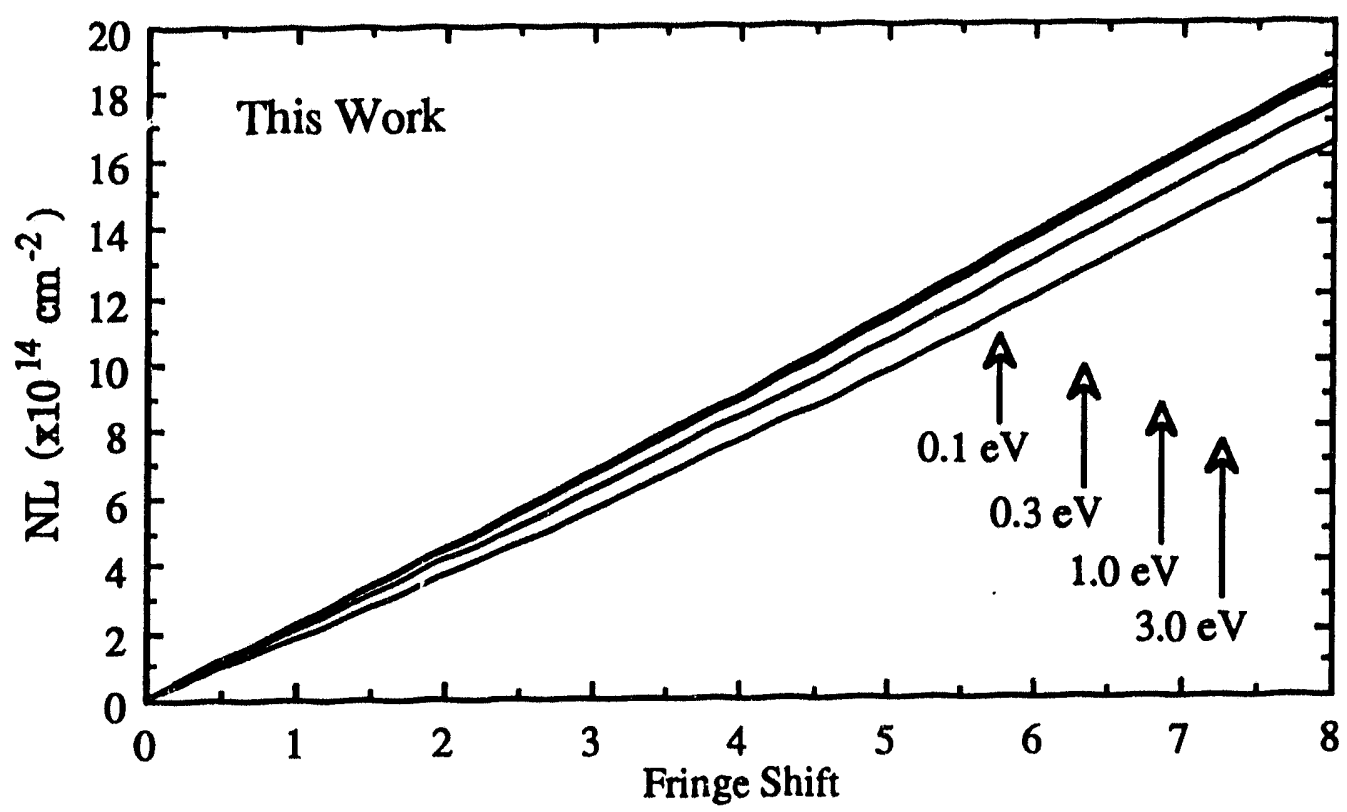

Fig. A3.14 This work's calculation at $0.009 \mathrm{~nm}$ for several ablation plume temperatures. 


\section{APPENDIX 4 TIME DELAY CIRCUITS}

The time delay between the Lumonics Laser and the Lambda Physik Excimer pumped Dye Laser was varied using one of two electronic arrangements. Figure A4.1 shows the timing circuit used for the holographic data presented in the text from October 1992 through April 1993, and Figure A4.2 shows the timing circuit used for the holographic data presented in the text for May through June 1993. The only difference is the Delay Generator setup (shown at the top of the figures).

The time delay between the two laser pulses was measured on the Tektronix DSA 602. Figure A4.3 shows an example of the recorded traces. For examples of the calorimeter signals, see Appendix 5. 


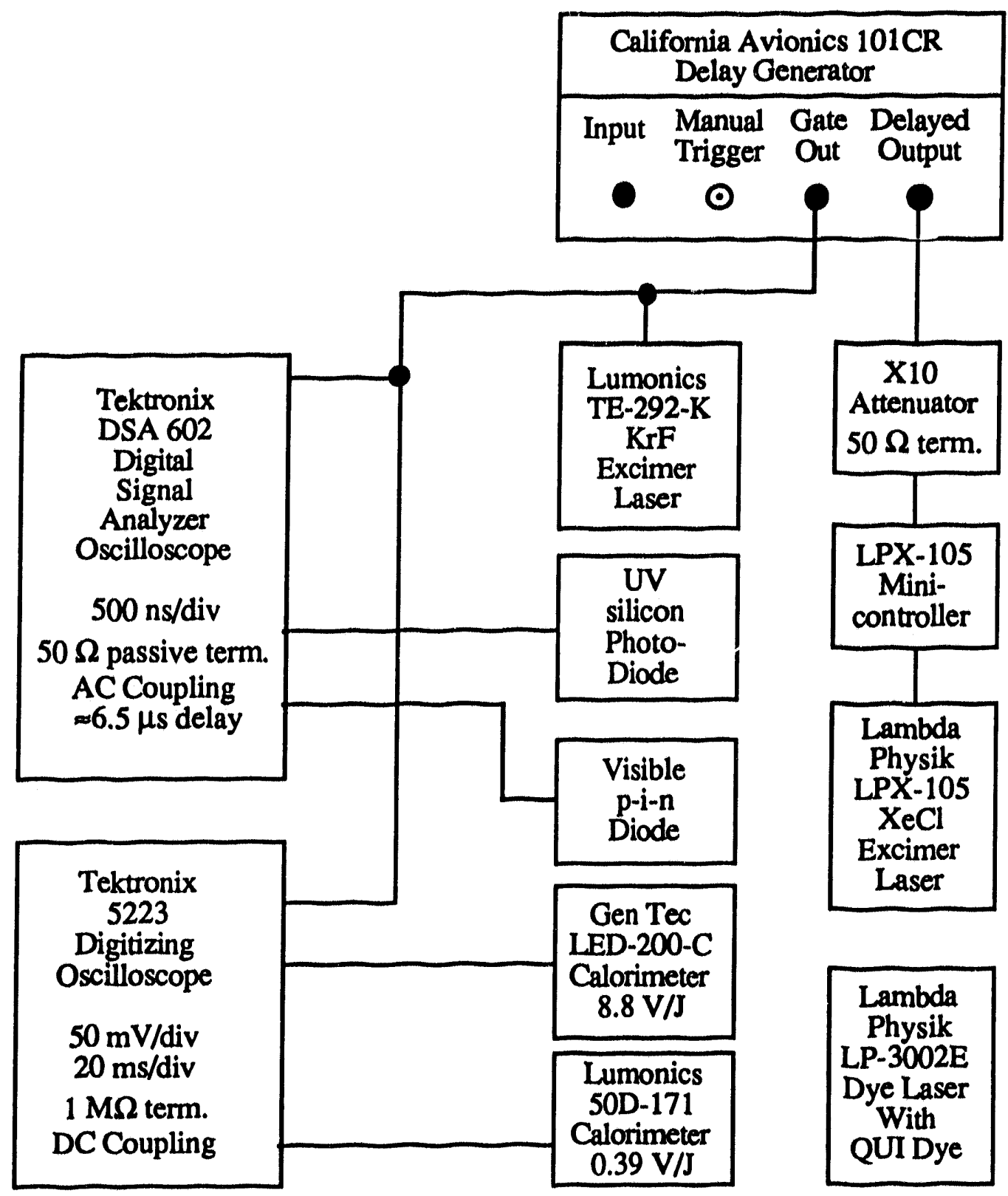

Fig. A4.1 Electrical schematic for July 1992 to April 1993. 


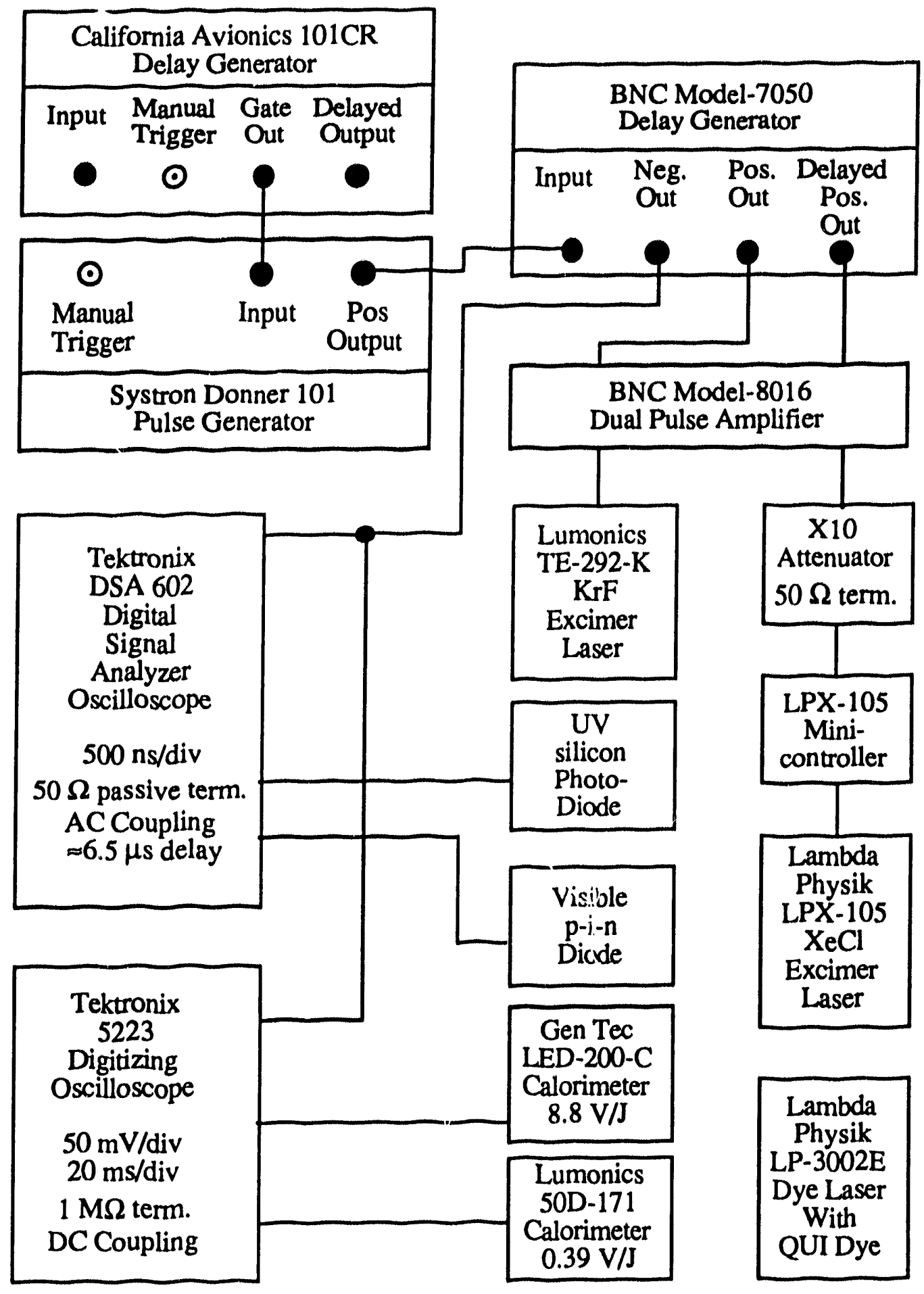

Fig. A4.2 Electrical schematic for May to June, 1993. 


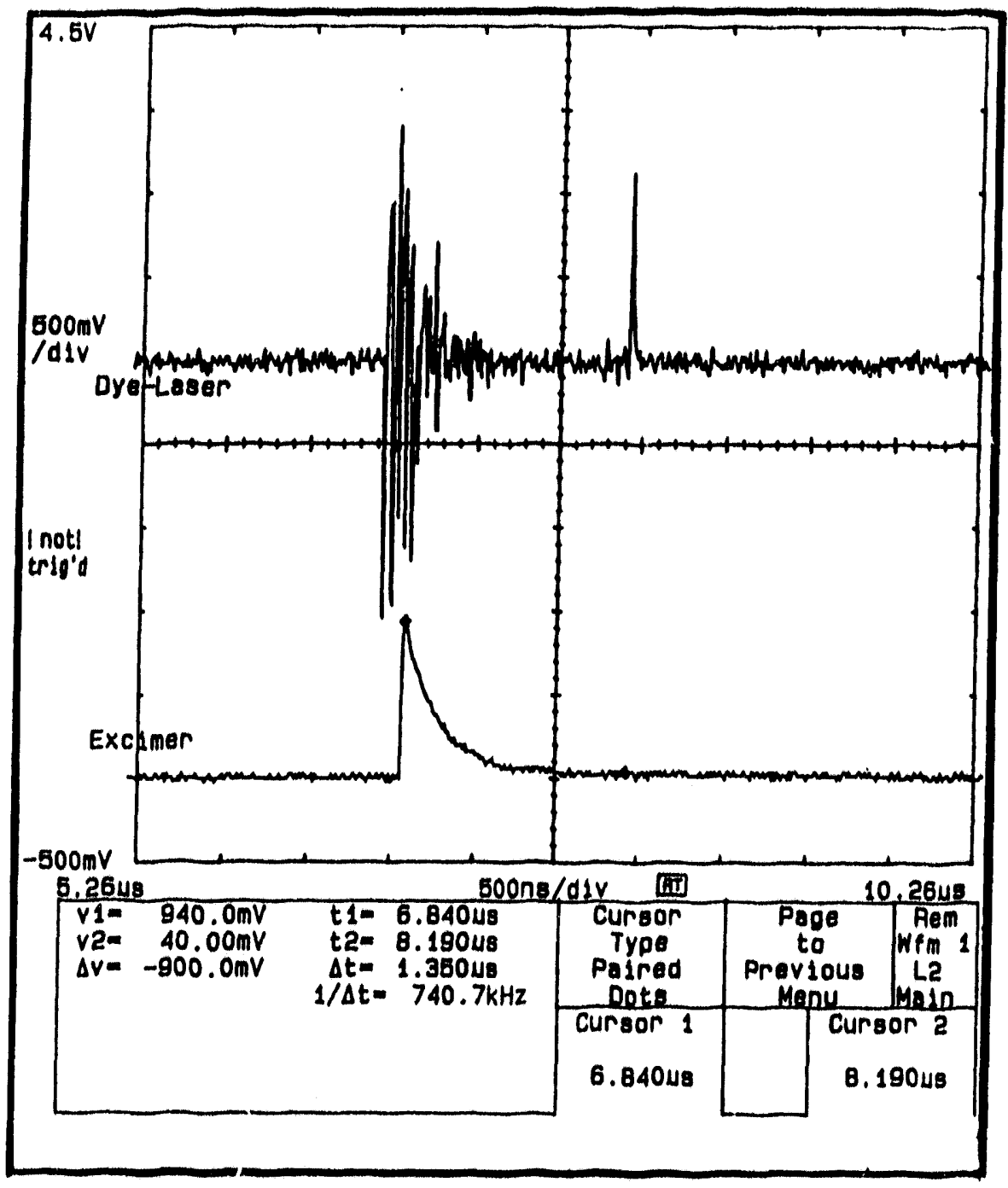

Fig. A4.3 Example of the timing signals as seen from the Digital Signal Analyzer (DSA) on a scale of $500 \mathrm{~ns} /$ div. The upper trace $(2 \mathrm{mV} / \mathrm{div})$ is the PIN diode signal. The isolated peak on the right side is the dye laser signal, while the noise at earlier times is due to the electromagnetic emission of the $\mathrm{KrF}$ laser internal electrical discharge. The lower trace $(500 \mathrm{mV} / \mathrm{div})$ is the silicon photo-diode signal. The initial edge of the of the signal represents the $\mathrm{KrF}$ laser pulse, while the long tail is either due to the fluorescence of the $\mathrm{KrF}$ laser beam focusing lens or to the supporting circuitry of the silicon photo-diode (RC decay). The time difference is determined by aligning a vertical bar with each of the laser peaks (here shown as two diamonds on the lower trace) and reading the DSA time difference below (here, $1.350 \mu \mathrm{s}$ ). 


\section{APPENDIX 5 \\ ENERGY CALIBRATION}

The shot-to-shot energy is monitored by splitting off part of the Lumonics laser beam onto a calorimeter, called the Side Calorimeter (Gen-Tec LED-200-C, $8.8 \mathrm{~V} / \mathrm{J}$, diameter $\approx 2 \mathrm{~cm}$ ). It is general practice to calibrate the signal from this calorimeter after each experimental run because: 1) the black, front surface of the Side Calorimeter has been irregularly ablated over its lifetime of use, and 2) because moving the focal lens changes the absorption spot on the Side Calorimeter as well as the ablation spot on the target. Figure 3.1(b) shows the experimental configuration for this calibration, where the Side Calorimeter is calibrated using a second calorimeter (Lumonics 50D-171, $0.39 \mathrm{~V} / \mathrm{J}$, diameter $\approx 11.5 \mathrm{~cm}$ ).

A Tektronix 5223 Digitizing Oscilloscope was used to record the calorimeter signals. Figure A5.1 shows an examples of the calorimeter traces during a calibration run. The peak signals are divided by the appropriate conversion factors (given above) to obtain the incident energy. Figure A5.2 shows an example calibration graph, where the side calorimeter energy is graphed versus the energy hitting the target (back calorimeter) (see Fig. 3.1(b)).

The same target spot on the same aluminum sample was used during the experimental runs during October 3 - 23, 1992 (see Fig. 3.2). Unfortunately, a side-to-back calibration was not performed after the last run and before the optical table components were disturbed. The energy calibration for the data taken in that time period was determined in the following way. It is a general practice to place the larger calorimeter in 


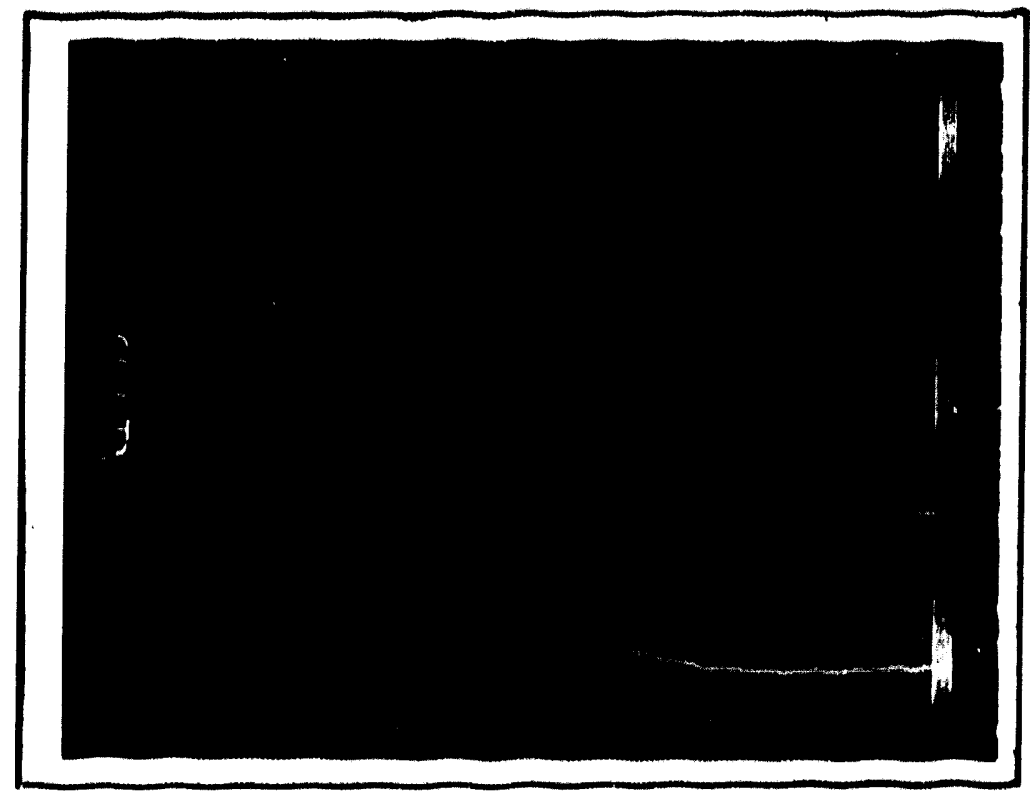

Fig. A5.1 Example of calorimetry signals as seen from the Tektronix 5223 Digitizing Oscilloscope on a scale of $20 \mathrm{~ms} / \mathrm{div}$. The top trace $(50 \mathrm{mV} / \mathrm{div})$ is the side calorimeter at position (C) on Fig. 3.1 (b) and the bottom trace $(50 \mathrm{mV} / \mathrm{div})$ is the second calorimeter placed at position (D). Note: the lower trace has the higher peak and undershoots after $\approx 55 \mathrm{~ms}$. 


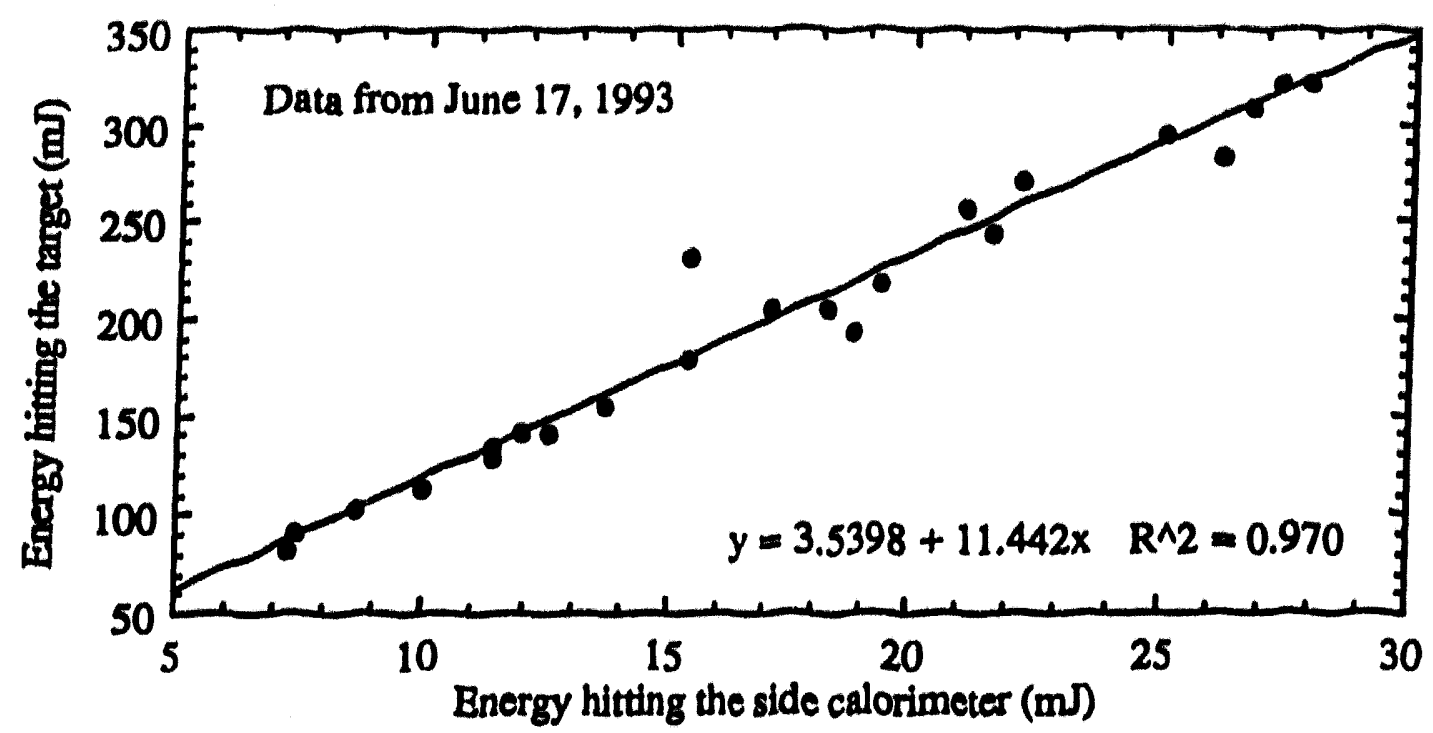

Fig. A5.2 Example fluence calibration of the side calorimeter (position C) versus the energy measured behind the chamber (position D): deposition in vacuum experiment, June 17, 1993; (see Fig. 3.1(b))

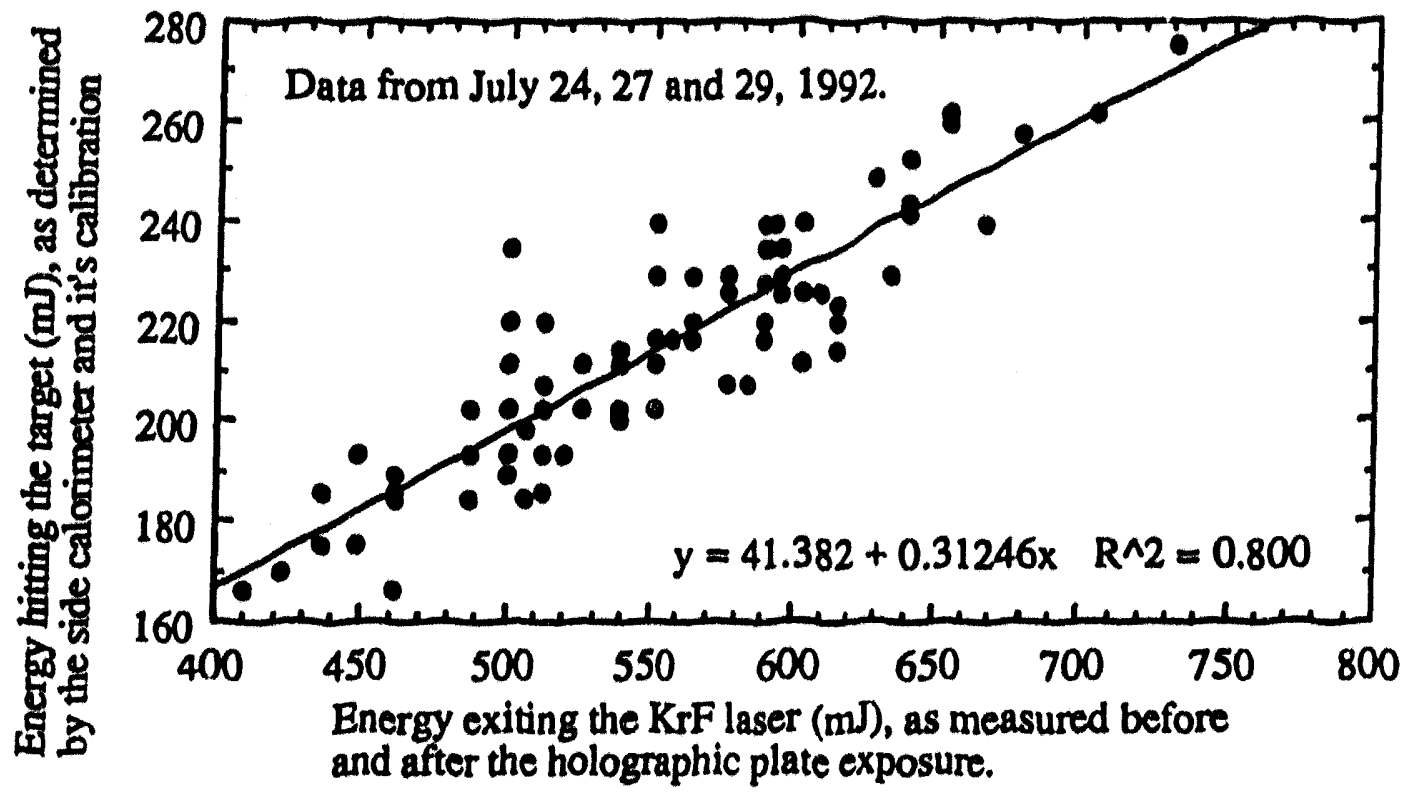

Fig. A5.3 Calibration of the energy measured behind the chamber (position D) to calibrated to the front of the chamber (position F); (see Fig. 3.1(b)). 
front of the focusing lens for a single shot both before and after taking a holographic plate for comparison with the total Lumonics laser output since the laser's purchase and installation. The larger calorimeter in this position is called the Front Calorimeter. In an experiment in July 1992, both Front and Side calorimetry signals were recorded while taking the holographic plates aid a Side-to-Back calibration was then performed. Figure A5.4 shows the Front-to-Back calibration curve determined from this July 1992 experiment and used with the October 1992 data as an approximation of the energy hitting the target. 


\section{APPENDIX 6 ANALYZING THE LASER ABLATION PLUME EXPANSION}

Figures A6.1 through A6.5 show the plume expansion analysis, as described in Chapter 4, for the holograms in Figures 4.6, 4.8, 4.12, 4.14, and 4.16. Each connected set of points represents one hologram, and each point represents the farthest extension of an equicontour line for the hologram. For the information derived from these graphs, see Figures $4.20,4.21,4.22$, and 4.23 . 


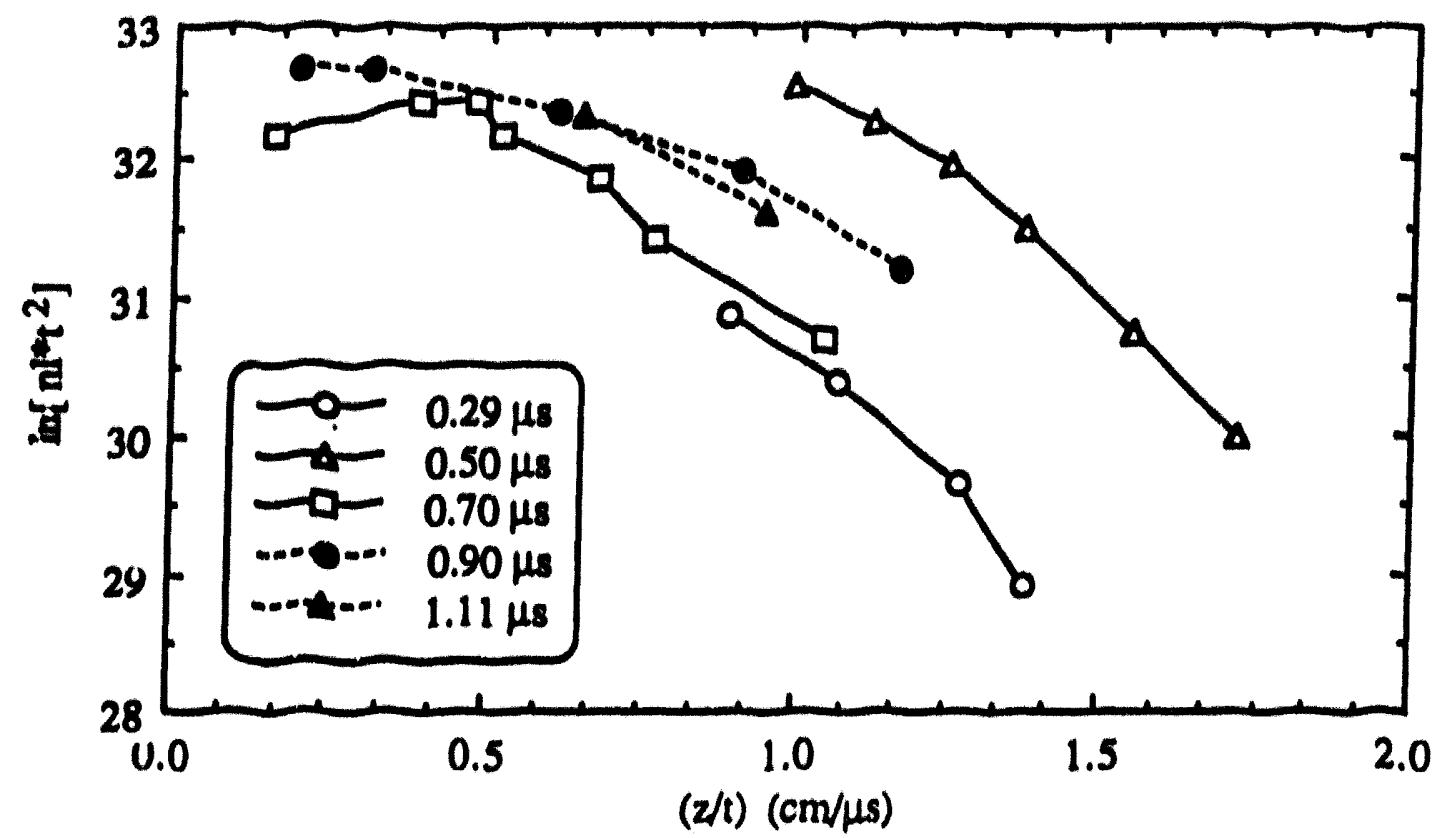

Fig. A6.1 Analysis of plume expansion in vacuum; for the holograms in Fig. 4.6.

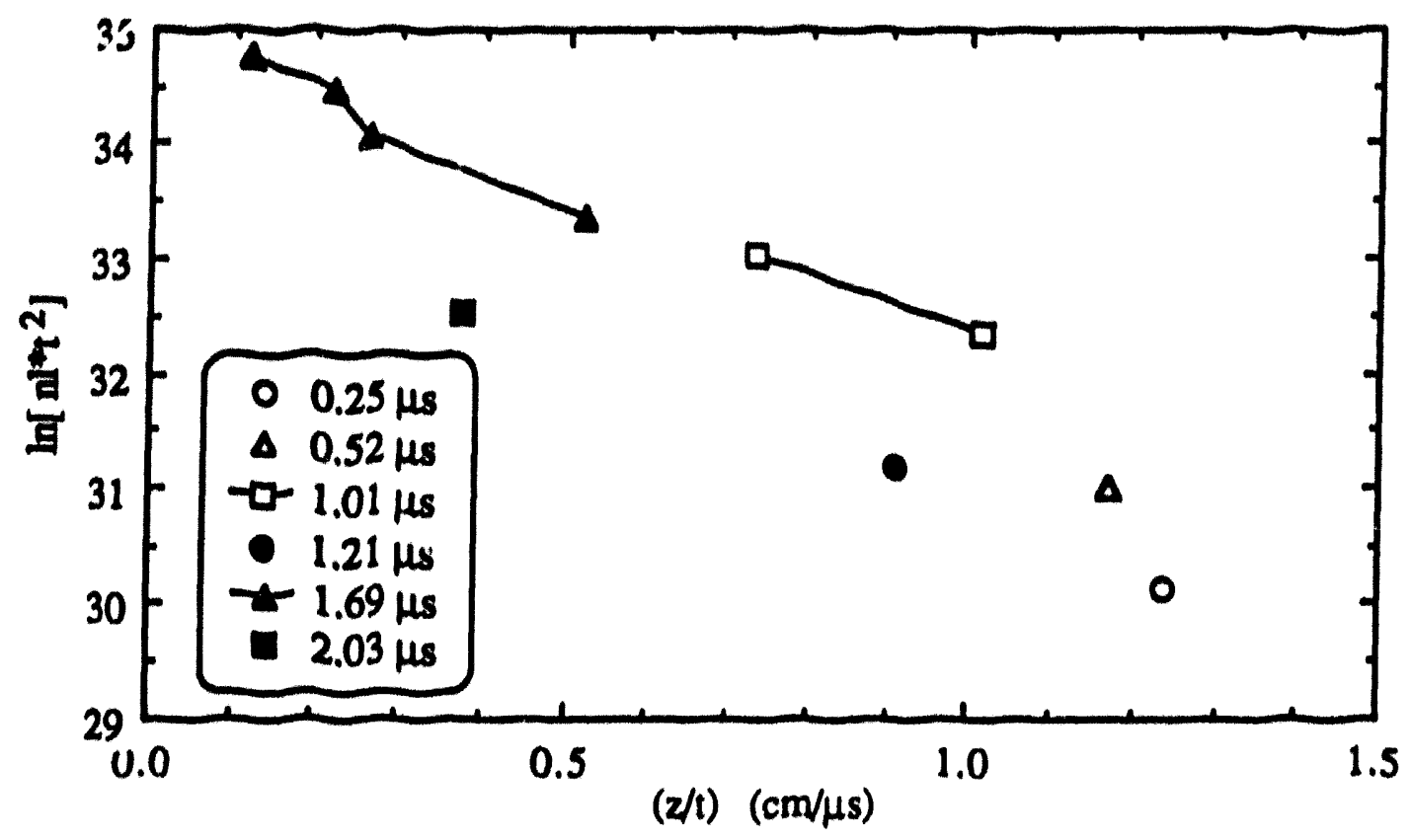

Fig. A6.2 Analysis of plume expansion in vacuum for the deposition case; for the holograms in Fig. 4.8. 


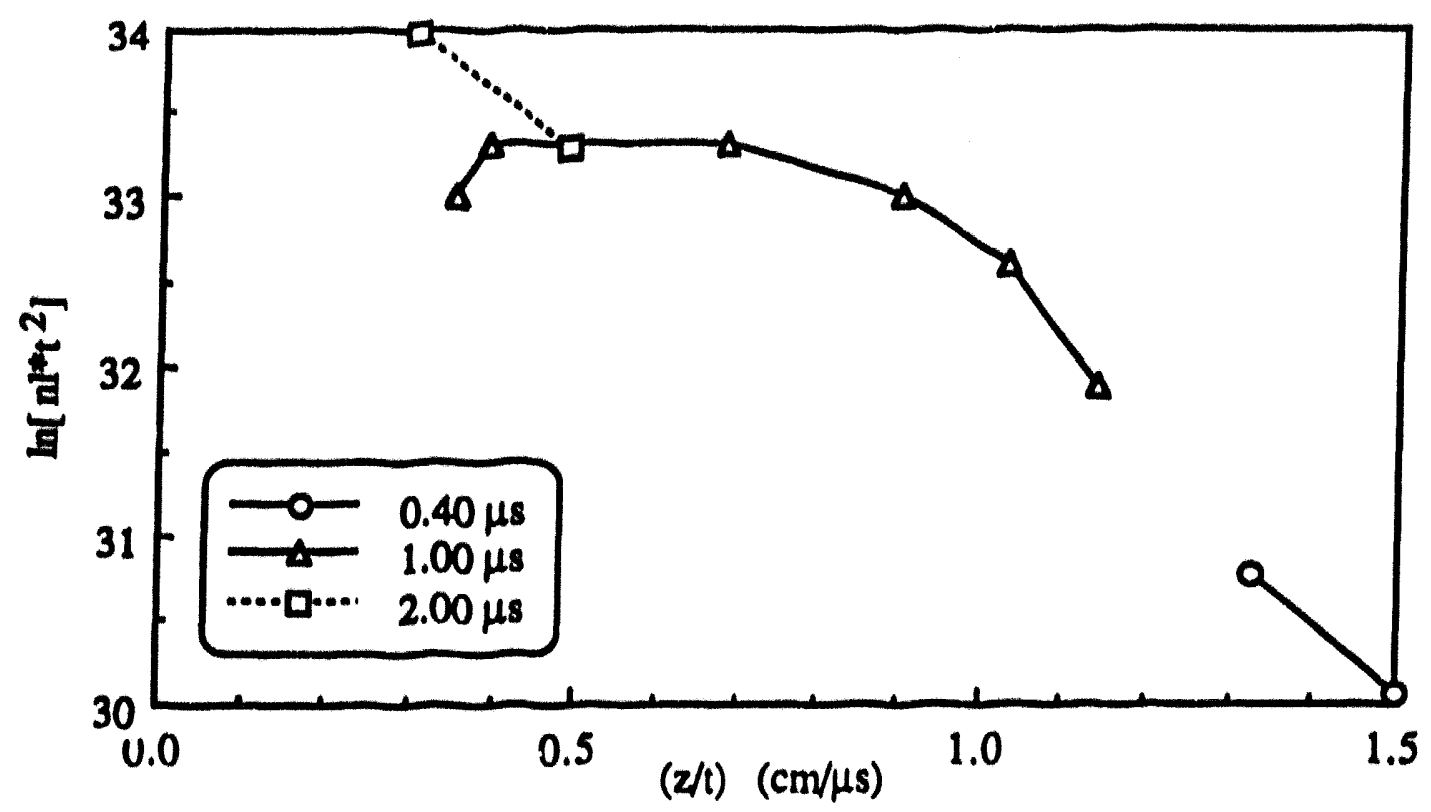

Fig. A6.3 Analysis of plume expansion in $14 \mathrm{mT}$ Torr argon gas; for the holograms in Fig. 4.12.

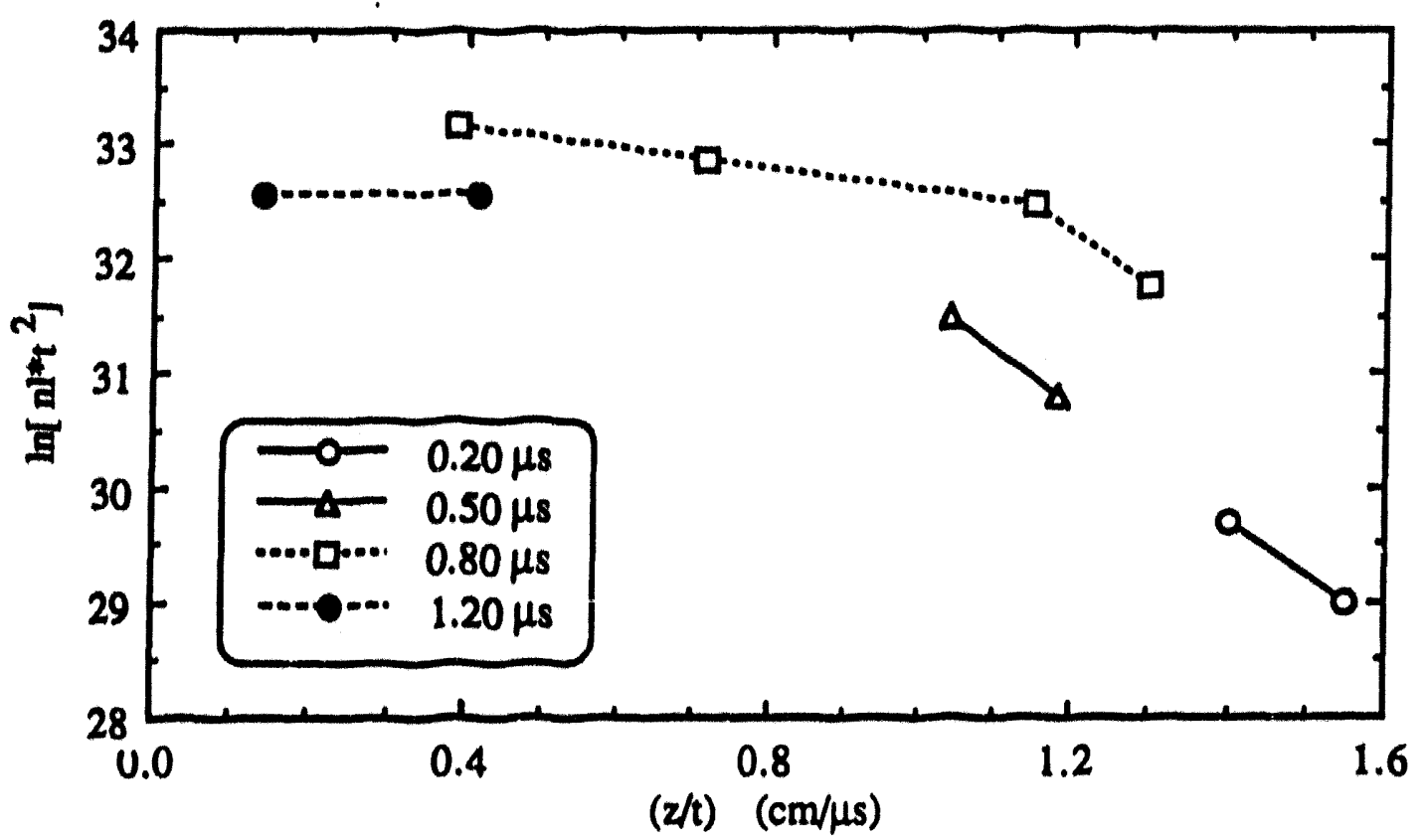

Fig. A6.4 Analysis of plume expansion in $52 \mathrm{mTorr}$ argon gas; for the holograms in Fig. 4.14. 


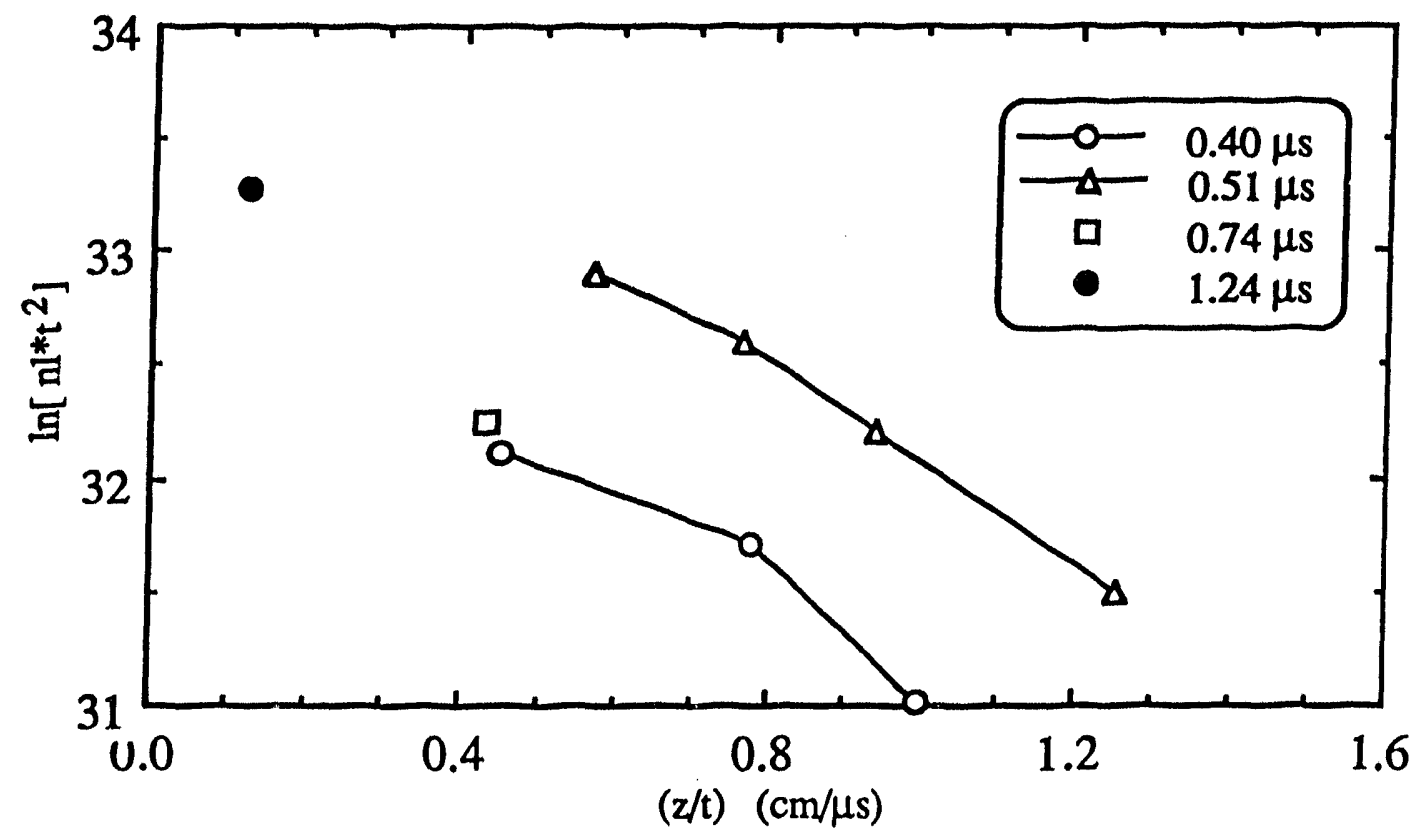

Fig. A6.5 Analysis of plume expansion in $210 \mathrm{mTorr}$ argon gas; for the holograms in Fig. 4.16. 


\section{BIBLIOGRAPHY}

163 


\section{BIBLIOGRAPHY}

Abr72 M. Abramowitz and I. Stegen, Eds., "Handbook of Mathematical Functions", Dover, New York, 1977.

Arf85 G. Arfken, "Mathematical Methods for Physicists, 3rd Edition", Academic Press, Inc., Harcourt Brace Jovanovich Publishers, San Diego, 1985.

Bac80 H.A. Bachor and M. Kock, "Limitations in the evaluation of hook spectra", J. Phys. B: Atom. Molec. Phys., 13, 2497 (1980).

Bak87 J.S. Bakos, P.N. Ignacz, and J. Szigeti, "Laser blow-off plasma propagating in low-pressure gas", Appl. Phys. Lett., 51, 734 (1987).

Ber71 D. Bershader, "Some aspects of the refractive behavior of gases", in "Modern optical methods in gas dynamic research", D.S. Dosanjh ed., Plenum Press, New YorkLondon (1971).

Bon86 D.J. Bone, H.-A. Bachor, and R.J. Sandeman, "Spectral line interferometry with temporal and spatial resolution", Optics Communication, 57, 39 (1986).

Bow85 J.L. Bowen and A.P. Thorne, "Time-resolved fluorescence and population measurements in laser-pumped barium vapor", J. Phys. B: At. Mol. Phys., 18, 35 (1985).

Bra87 M.L. Brake, J. Meachum, R.M. Gilgenbach, and W. Thomhill, "Temporally resolved spectroscopy of the laserinduced carbon ablation plasmas", IEEE Trans. Plasma Sci., PS-15, (1987).

Bra91 J.H. Brannon, A.C. Tam, and R.H. Kurth, "Pulsed laser stripping of polyurethane-coated wires: A comparison of $\mathrm{KrF}$ and $\mathrm{CO}_{2}$ lasers", J. Appl. Phys. 70, 3881 (1991).

Bro93 C. Brooks and M.L. Brake, Private Communication, 1993. 
Cap90 M.A. Cappelli, P.H. Paul, and R.K. Hanson, "Laser-induced fluorescence of laser-ablated barium", Appl. Phys. Lett. 56, 1715 (1990).

Che91 N.H. Cheung, Q.Y. Ying, J.P. Zheng and H.S. Kwok, "Time-resolved resonant absorption study of 532-nm lasergenerated plumes over YBCO targets", J. Appl. Phys., 69, 6349 (1991).

Co192 C.B. Collins, F. Davanloo, T.J. Lee, D.R. Jander, J.H. You, H. Park and J.C. Pivin, "The bonding of protective films of amorphic diamond to titanium", J. Appl. Phys. 71, 3260 (1992).

Con89 R.J. Contolini and J. Alspector, "Fine line aluminum etching in air and in solution using a tunable dye laser", J. App. Phys. 65, 1759 (1989).

Cra86 J.E. Craig, M. Azzazy, and C.C. Poon, "Resonant holographic detection of hydroxyl radicals in reacting flows", AIAA Journal, 24, 74 (1986).

Cro91 H. Cronberg, M.Reichling. E. Broberg, H.B. Nielsen, E. Matthias, and N. Tolk, "Effects of inverse Bremsstrahlung in laser-induced plasmas from a graphite source", Appl. Phys. B, 52, 155 (1991).

Dic90 J.T. Dickinson, S.C. Langford, L.C. Jensen, P.A. Eschbach, L.R. Pederson and D.R. Baer, "Consequences of simultaneous exposure of inorganic solids to excimer laser light and an electron beam", J. Appl. Phys., 15, 1831 (1990).

Dre71 G.V. Dreiden, Y.I. Ostrovsky, E.N. Shedova, and A.N. Zaidel, "Holographic interferograms in stimulated raman light", Optics Communication, 4, 209 (1971).

Dre74 G.V. Dreiden, A.N. Zaidel, Y.I. Ostrovskii and E.N. Shedova, "Three wavelength hologram diagnostics of an optical burst on a potassium target", Sov. Phys. Tech. Phys., 18, 972 (1974). 
Dre75 G.V. Dreiden, A.N. Zaidel', G.V. Ostrovskaya, Yu.I. Ostrovskii, N.A. Pobedonostseva, L.V. Tanin, V.N. Filippov, and E.N. Shedova, "Plasma diagnostics by resonant inteferometry and holography", Sov. J. Plasma Phys., 1, 256 (1975).

Dre86 R.W. Dreyfus, R. Kelly., and R.E. Walkup, "Laser-induced fluorescence studies of excimer laser ablation of AlO", Appl. Phys. Lett., 49, 1478 (1986).

Dre91 R.W. Dreyfus, "Cuo, $\mathrm{Cu}^{+}, \mathrm{Cu}_{2}$ from excimer laser-ablated copper", J. Appl. Phys., 69, 1721 (1991).

En187 C.L. Enloe, R.M. Gilgenbach, and J.S. Meachum, "Fast, sensitive laser deflection system suitable for transient plasma analysis", Rev. Sci. Instrum., 58, 1597 (1987).

En188 C.L. Enloe, "Ultraviolet-induced flashover of the highlyangled polymeric insulators in vacuum", Ph.D. Thesis, The University of Michigan, 1988.

Elt90 R.C. Elton, "X-Ray Lasers", Academic Press, Boston, 1990.

Eri

K.B. Erington, W. McGahan, N.J. Ianno, and J.A. Woollam, "Pulsed laser deposition of magneto-optic material", Dept. of Electrical Engineering, Univ. of Nebraska-Lincoln, NE (unpublished).

Fel87 D. Feldman, J. Kutzner, J. Laukemper, S. MacRobert, and K.H. Welge, "Mass-spectroscopic studies of the ArF-laser photo-ablation of polystyrene", Appl. Phys. B, 44, 81 (1987).

Fog90 E. Fogarassy, C. Fuchs, A. Slaoui, and J.P. Stoquert, "SiO thin-film deposition by excimer laser ablation from Sio target in oxygen atmosphere", Appl. Phys. Lett., 57, 664 (1990).

Geo89 D.B. Geohegan a.su D.N. Mashburn, "Characterization of ground-state neutral and ion transport during laser ablation of YBCO using transient optical absorption spectroscopy", Appl. Phys. Lett., 55, 2345 (1989). 
Ge092 D.B. Geohagen, "Fast intensified-CCD photography of YBCO laser ablation in vacuum and ambient oxygen", Appl. Phys. Lett., 60, 2732 (1992).

Ge093 D. B. Geohagen, "Imaging and blackbody emission spectra of particulates generated in the KrF-laser ablation of $\mathrm{BN}$ and YBCO", Appl. phys. Lett., 62, 1463 (1993).

Gi191 R.M. Gilgenbach and P.L.G. Ventzek, "Dynamics of excimer laser-ablated aluminum neutral atom plume measured by dye laser resonance absorption photography", Appl. Phys. Lett., 58, 1597 (1991).

Goo85 J.W. Goodman, "Statistical Optics", Wiley-Interscience Publications, N.Y., 1985.

Gri64 H.R. Griem, "Plasma Spectroscopy", McGraw-Hill, New York, 1964.

Gup91 A.Gupta, B. Braren, K.G. Casey, B.W. Hussey, and R. Kelly, "Direct imaging of the fragments produced during excimer laser ablation of YBCO", Appl. Phys. Lett., 59, $1302(1991)$

Gut89 R.J. von Guttfield and R.W. Dreyfus, "Electronic probe measurements of pulsed copper ablation at $248 \mathrm{~nm}$ ", Appl. Phys. Lett., 54, 1212 (1989).

Hec79 E. Hecht and A. Zajac, "Optics", Addison-Wesley, Reading, MA, 1979.

Jel81 G. Jellison and C.R. Parsons, "Resonant shadowgraph and schlieren studies of magnetized laser produced plasmas", Phys. Fluids, 24, 1787 (1981).

Kar92 H. Karl and B. Stritzker, "Reflection high-energy electron diffraction oscillations modulated by laser-pulse deposited YBCO", Phys. Rev. Lett., 69, 2939 (1992).

Kel85 R. Kelly and J.E. Rothenburg, "Laser sputtering part 3. The mechanism of the sputtering of metals at low energy densities", Nuc. Instrum. and Methods in Phys. Research, B7/8, 755 (1985). 
Kel88 R. Kelly and R.W. Dreyfus, "Reconsidering the mechanisms of laser sputtering with Knudsen-layer information taken into account", Nuc. Instrum. and Methods in Phys. Research", B32, 341 (1988).

Koi91 H. Koinuma, H. Nagata, T. Tsukahara, S. Gonda, "Ceramic layer epitaxy by pulsed laser deposition in an ultrahigh vacuum system", Appl. Phys. Lett. 58, 2027 (1991).

Koo92 J.C.S. Kools, T.S. Baller, S.T. De Zwart, and J. Dieleman, "Gas flow dynamics in laser ablation deposition", J. Appl. Phys., 71, 4547 (1992).

Koo78 D.K. Koopman, H.-J. Siebeneck, G. Jellison, and W.G. Niessen, "Resonant holography of plasma flow phenomena", Rev. Sci. Instrum., 49, 524 (1978).

Koo79 [I.K. Koopman, H.-J. Siebeneck, and G. Jellison, "Turbulent interaction fronts in counterstreaming laser-produced plasma studies", Phys. Fluids, 22, 526 (1979).

Kre93 H.-U. Krebs and O. Bremart, "Pulsed laser deposition of thin metallic alloys", Appl. Phys. Lett. 62, 2341 (1993).

Küg83 E. Kügler and D. Bershader, "Recent high-resolution resonant refractivity studies of a sodium-seeded flame", Exp. in Fluids, 1, 51 (1983).

Kwo88 H.S. Kwok, P. Mattocks, L. Shi, X.W. Wang, S.

Witanachchi, Q.Y. Ying, J.P. Zheng, and D.T. Shaw, "Laser evaporation depression of superconducting and dielectric thin films", Appl. Phys. Lett., 52, 1825 (1988).

Lan87 S.C. Langford, J.T. Dickinson, and L.C. Jensen, "Simultaneous measurements of the electron and photo emission accompanying fracture of single-crystal $\mathrm{MgO}$ ", $\mathrm{J}$. Appl. Phys., 62, 1437 (1987).

Lin93 R.A. Lindley, R.M. Gilgenbach, and C.H. Ching, "Resonant holographic interferometry of laser-ablation plumes", Appl. Phys. Lett., 63, 888 (1993). 
Lid90 D. R. Lide, Ed., "CRC Handbook of Physics and Chemistry", CRC Press, Boca Raton, FL, 1990.

Liu92 R.S. Liu, W.C. Shih, K. Scott, P.P. Edwards, W.A. Phillips, and A.L. Greer, "Preparation and characterization of $\mathrm{TiBaCaCuo}$ films by laser ablation and thallium diffusion", J. Appl. Phys., 71, 4085 (1992).

Mar6'/ W.C. Marlow, "Hakenmethode", Appl. Optics, 6, 1715 (1967).

Mea70 R.M. Measures, "Spectral line interferometry: a proposed means of selectively measuring the change in the density of a specific atom population", Appl. Optics, 9, 737 (1970).

Mea88 J.S. Meachum, "Neutral Beam Attenuation in Laser Ablated Atoms and Plasma", Ph.D. Dissertation, University of Michigan, Ann Arbor, MI, 1988.

Mio92 A. Miotello, R. Kelly, B. Braren, and C.E. Otis, "Novel geometrical effects observed in debris when polymers are laser sputtered", Appl. Phys. Lett., 61, 2784 (1992).

Mos88 A.N. Mostovych, B.H. Ripin, and J.A. Stamper, "Resonant diagnostics of laser-produced Ba plasmas", Rev. Sci. Instrum., 59, 1497 (1988).

Nay85 M.H. Nayfeh and M.K. Brussel, "Electricity and Magnetism", Wiley, New York, 1985.

Niu92 C. Niu, C.M. Lieber, "Low-temperature growth of the infinite layer phase of $\mathrm{SrCuO}$ by pulsed laser deposition", J. Am. Chem. Soc., 114, 3570 (1992).

Noo87 I. NoorBatcha, R.R. Lucchese and Y. Zeiri, J. Chem. Phys. 86, 5816 (1987).

Nor91 M.G. Norton, P.G. Kutula, and C.B. Carter, "Oriented aluminum nitride thin films deposited by pulsed-laser deposition", J. Appl. Phys., 70, 2871 (1991). 
Ode80 H.J. Odenthal and J. Uhlenbusch, "Determination of temperature, velocity distribution, and population densities of neutral helium by means of laser light tuned on atomic resonances", IEEE Transactions on Plasma Sci., PS-8, 431 (1980).

Oka92 T. Okada, Y. Nakayama, W.K.A. Kum ınduni, and M. Maeda, "Observation of scattering of particles produced by laser ablation on a substrate by laser induced fluorescence", Appl. Phys. Lett., 61, 2368 (1992).

Ost75 G.V. Ostrovskaya and N.A. Pobedonostseva," Determinination of the density of excited hydrogen atoms in a laser spark by resonant interferometry", Sov. Phys. Tech. Phys., 20, 923 (1975).

Ost75 Y.I Ostrovsky and L.V. Tanin, "Tunable organic dye laser for resonance interferometry and holography", Sov. Phys. Tech. Phys., 20, 1118 (1975).

Ost85 G.V. Ostrovskaya and Y. I. Ostrovsky, "Holographic methods of plasma diagnostics", Progress in Optics, 22, 197 (1985).

Pen93 J. Pender and A. Selvakumar, Private Communication, 1993.

Pet88 S. Petzoldt, A.P. Elg, M. Reichling, J. Reif, and E. Matthias, "Surface laser damage thresholds determined by photoacoustic deflection", Appl. Phys. Lett., 53, 2005 (1988).

Qia93 F. Qian, V. Nagabushnam, R.K. Singh, "Pulsed laser deposition of cubic boron nitride films", Appl. Phys. Lett. 63, 317 (1993).

Raj93 D. Rajavel and J.J. Zinck, "Metal-organic molecular beam

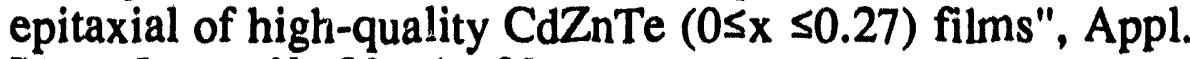
Phys. Lett., 63, 322 (1993).

Rot84a J.E. Rothenberg and R. Kelly, "Laser sputtering part 2. The mechanism of the sputtering of $\mathrm{AlO}$ ", Nuclear Instrum. and Methods in Phys. Research, B1, 291 (1984). 
Rot84b J.E. Rothenberg and G. Koren, "Laser produced plasma in crystalline $\alpha-\mathrm{AlO}$ and aluminum metal", Appl. Phys. Lett., 44, 664 (1984).

Roy92 D. Roy and S.B. Krupanidhi, "Pulsed excimer laser ablated barium titanate thin films", Appl. Phys. Lett., 61, 2057 (1992).

Rub91 L.F. Rubin and D.M. Swain, "Near-resonant holography", Optics Lett., 16, 526 (1991).

Sae91 K. L. Saenger, "On the origin of spatial nonuniformities in the composition of pulsed-laser-deposited films", J. Appl. Phys., 70, 5029 (1991).

Sap91 A.D. Sappey and T.K. Gamble, "Laser-fluorescence diagnostics for condensation in laser-ablated copper plasmas", Appl. Phys. B, 53, 353 (1991).

Sap92 A.D. Sappey and T.K. Gamble, "Planar laser-induced fluorescence imaging of $\mathrm{Cu}$ atom and $\mathrm{Cu}_{2}$ in a condensing laser-ablated copper plasma plume", J. Appl. Phys., 72, 5095 (1992).

Sap93 A.D. Sappey, T.K. Gamble, and D.K. Zerkle, "Absolute copper atom density determination in laser-ablated copper plasmas using hook spectroscopy", Appl. Phys. Lett. 62, 564 (1993).

Sat88 T. Sato, S. Furuno, S. Iguchi, and M. Hanabusa, "Diamondlike carbon films prepared by pulsed-laser evaporation", Appl. Phys. A, 45, 355 (1988).

Sch68 H. Schwartz and H.A. Tourtellotte, "Vacuum deposition by high-energy laser with emphasis on barium titanate films", J. Vacuum Sci. and Tech., 6, 373 (1968).

Se191 J. A. Sell, D.M. Heffelfinger, P.L.G. Ventzek, and R.M. Gilgenbach, "Photoacoustic and photothermal beam deflection as a probe of laser ablation of materials", I. Appl. Phys., 69, 1330 (1991). 
Sie77 H.-J. Siebeneck, D.K. Koopman, and J.A. Cobble, "Resonant optical schlieren system for plasma flow studies", Rev. Sci. Instrum., 48, 997 (1977).

Sie86 A.E. Siegman, "Lasers", University Science Books, Mill Valley, CA, 1986.

Sri84 R. Srinivasan and B. Braren, "Ablative photo- decomposition of polymer films by pulsed far-ultraviolet $(193 \mathrm{~nm})$ laser radiation: dependence of etch depth on experimental conditions", J. of Polymer Sci., 22, 2601 (1984).

Sri93 R. Srinvisan, "Ablation of polymethyl methacrylate films by pulsed (ns) ultraviolet and infrared $(9.17 \mu \mathrm{s})$ lasers: a ccimparative study by ultrafast imaging", J. Appl. Phys., 73, 2743 (1993).

Sta88 J.D. Stafford, and J.A. Holcombe, "Time-gated resonance sohlieren studies of analyte distribution in a graphite furnace atcmiser", J. of Analytical Atomic Spectrometry, 3, 35 (198Z).

Tal83 G.J. Tallents, "Two-dimensional ion emission from laserproduced plasmas", Laser and Particle Beams, 1, 171 (1983).

Tar93 E.J. Tarsa, J.H. English, and J.S. Speck, "Pulsed laser deposition of oriented InO on (001) InAs, $\mathrm{MgO}$, and yttriastabilized zirconia", Appl. Phys. Lett., 62, 2332 (1993).

Utt76 N.G. Utterback, S.P.Tang, J.F. Friichtenicht, "Atomic and ionic beam source utilizing pulsed laser blow off", Phys. Fluids, 19, 900 (1976).

Ven88 T.Venkatesan, X.D. Wu, A. Inam, and J.B. Wachtman, "Observations of two distinct components during pulsed laser deposition of high $\mathrm{T}_{\mathfrak{c}}$ superconducting films", Appl. Phys. Lett. 53, 1193 (1988).

Ven90 P.L.G. Ventzek, R.M. Gilgenbach, J. A. Sell, and D.M. Heffelfinger, "Schlieren measurements of the hydrodynamics of excimer laser ablation of polymers in atmospheric pressure gas", J. Appl. Phys., 68, 965 (1990). 
Ven92a P.L.G. Ventzek, R.M. Gilgenbach, C.H. Ching, and R.A. Lindley, "Schlieren and dye laser resonance absorption photographic investigations of $\mathrm{KrF}$ excimer laser-ablated atoms and molecules from polyimide, polyethyleneterephthalate, and aluminum", J. Appl. Phys., 72, 1696 (1992).

Ven92b P.L.G. Ventzek, R.M. Gilgenbach, C.H. Ching, and R.A. Lindley and W.B. McColl, "Copper vapor laser machining of polyimide and polymethlymethacrylate in atmospheric pressure air", J. Appl. Phys., 72, 3080 (1992).

Ves79 C. M. Vest, "Holographic Interferometry", Wiley, New York,1979.

Wa186 R.E. Walkup, J.M. Jasinski, and R.W. Dreyfus, "Studies of excimer laser ablation of solids using a Michelson interferometer", Appl. Phys. Lett., 48, 1690 (1986).

Wan91 H. Wang, A.P. Satzberg, and B. R. Weiner, "Laser ablation of aluminum at 193, 248, and $381 \mathrm{~nm} "$, Appl. Phys. Lett. 59, 935 (1991).

Wan92 L. Wang, K.W.D. Ledingham, C.J. McLean, and R.P. Singhal, "Laser-induced collisional processes in resonant laser ablation of GaAs", Appl. Phys. B, 54, 71 (1992).

Woi91 J. Woisetschlager, H. Jager, G. Pfeiffer, and T. Neger, "Tomographic investigation of the particle density distribution of sodium atoms in a glow discharge using heterodyne holographic interferometry", Phys. Lett. A, 152, 42 (1991).

Woi92 J. Woisetschlager, H. Jager, T. Neger, and K. Widmann, "Investigation of the population inversion in a He-Ne discharge by heterodyne holographic interferometry", Appl. Phys. B, 54, 132 (1992).

Zhe89 J.P. Zheng, Z.Q. Huang, D.T. Shaw, and H.S Kwok, "Generation of high-energy atomic beams in lasersuperconducting target iveractions", Appl Phys. Lett., 54, 280 (1989). 
Zhid93 S. Zhu, D.H. Lowndes, B.C. Chakoumakos, J.D. Budai, D.K.Christen, X.-Y. Zheng, E. Jones, B. Warmack, "In situ growth of epitaxial $\mathrm{BiSrCaCuO}$ and $\mathrm{BiSrCuO}$ films by pulsed laser ablation", Appl. Phys. Lett. 63, 409 (1993). 

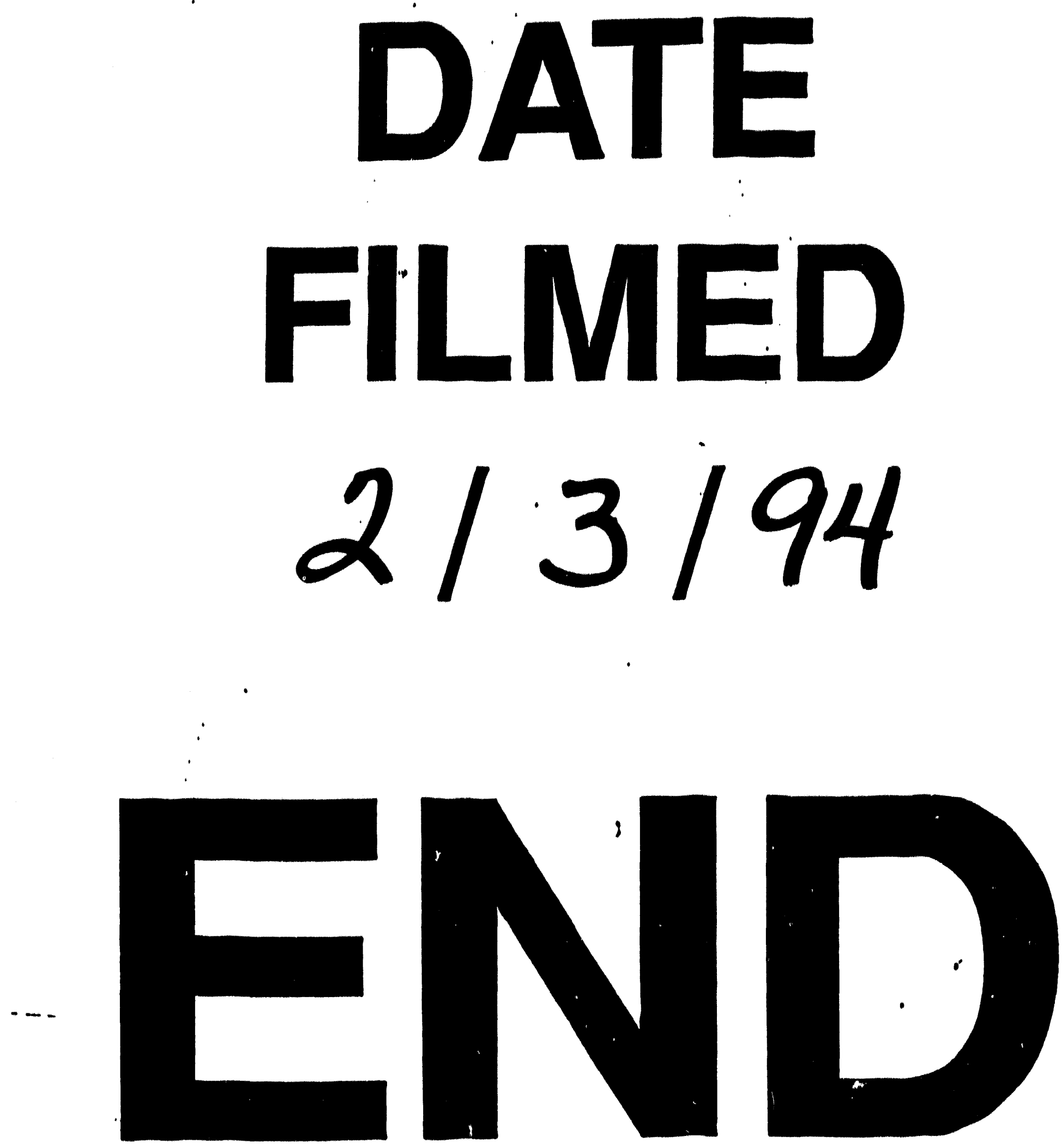